\title{
Visualisieren des Mikro- und Makromischens mit Hilfe zweier fluoreszierender und chemisch reagierender Farbstoffe
}

\author{
Vom Fachbereich Maschinenbau \\ der Universität Hannover \\ zur Erlangung des akademischen Grades \\ Doktor-Ingenieurin \\ genehmigte \\ Dissertation \\ von \\ Dipl.-Ing. Kerstin Kling \\ geboren am 10. April 1974 in Bückeburg
}


1. Referent: Prof. Dr.-Ing. D. Mewes

2. Referent: Prof. Dr.-Ing. Dr.-Ing. E.h. F. Mayinger

Vorsitzender der Prüfungskommission: Prof. Dr.-Ing. G. P. Merker Tag der Promotion: 10. Dezember 2004 


\section{Vorwort}

Diese Arbeit entstand während meiner Tätigkeit als wissenschaftliche Mitarbeiterin am Institut für Verfahrenstechnik der Universität Hannover. Mein besonderer Dank gilt dem Leiter des Instituts und meinem Doktorvater Herrn Prof. Dr.-Ing. Dieter Mewes. Er gewährte mir die nötigen Freiräume für meine Forschung und hatte stets großes Vertrauen in meine Arbeit. Dank seiner wissenschaftliche Anleitung und Förderung habe ich sowohl fachlich als auch persönlich viel gelernt.

Herrn Prof. Dr.-Ing. Dr.-Ing. E.h. Franz Mayinger danke ich für die Übernahme des Korreferats und Herrn Prof. Dr.-Ing. Günter P. Merker für die Übernahme des Prüfungsvorsitzes. Beiden sei an dieser Stelle für das Interesse an meiner Arbeit und die hilfreichen Gespräche gedankt.

Der Deutschen Forschungsgemeinschaft danke ich für die finanzielle Unterstützung der Arbeit.

Meine Kolleginnen und Kollegen haben durch Ihre Unterstützung und die freundschaftliche Atmosphäre am Institut zu einem fruchtbaren und angenehmen Arbeitsklima beigetragen. Danke besonders an Florian Schmidt, Bastian Mahr, Karijm Salem, Marc Lörcher und Sabine Luther, die nicht nur in fachlichen Fragen sondern auch im privaten Bereich stets ein offenes Ohr hatten. Zahlreiche studentische Hilfskräfte haben einen wesentlichen Beitrag zu dieser Arbeit geleistet. Hierbei möchte ich besonders Steffen Bütehorn und Markus Lachner erwähnen, die sich durch hohen Einsatz hervorgetan haben. Den technischen Mitarbeitern des Instituts, insbesondere Herrn Schickedanz, Herrn Schulz und Herrn Siedenberg, danke ich für Ihre wertvolle Unterstützung beim Aufbau meiner Versuchsanlage. Herrn Streichert sei für seinen Einsatz bei der Administration des Rechnernetzwerks und Frau Sladek und Frau Greiser für die organisatorische Hilfe gedankt.

Meiner Familie danke ich für Ihre Geduld und die stets große Unterstützung. Ein besonderer Dank gilt dabei meinem Freund, Markus André, der mir jederzeit mit Rat und Tat zur Seite stand und so erheblich zum erfolgreichen Abschluss der Arbeit beitrug. 


\section{Kurzfassung}

Kling, Kerstin

\section{Visualisieren des Mikro- und Makromischens mit Hilfe zweier fluoreszierender und chemisch reagierender Farbstoffe}

Für den Ablauf einer chemischen Reaktion ist das vollständig Vermischen der Reaktanden im molekularen Bereich der Ortsskalen notwendig. Zahlreiche Verfahren zum Messen der Mikrovermischung setzten eine ideale Makromischung voraus, während des laminaren Mischvorgangs treten aber stark unterschiedliche lokale Konzentrationen im Behältervolumen auf. In der vorliegenden Forschungsarbeit werden deshalb simultan das Vermischen auf makroskopischen Längenskalen sowie die Zusammensetzung fluider Gemische im Skalenbereich molekularer Abmessungen visualisiert. Dazu wird ein Gemisch aus zwei Fluoreszenzfarbstoffen in den Behälter dosiert. Der eine Farbstoff ist inert und dient als Indikator für das Makromischen. Die Fluoreszenzintensität des anderen, reagierenden Farbstoffs wird durch eine chemische Reaktion verstärkt. Da die Reaktion eine Vermischung auf molekularer Ebene voraussetzt, dient der reagierende Farbstoff indirekt dem Visualisieren der Mikrovermischung.

Die Konzentrationsfelder der Farbstoffe werden simultan mit Hilfe der Zwei-Farben Laserinduzierten Fluoreszenz (LIF) mit hoher zeitlicher und örtlicher Auflösung gemessen. Die Fluoreszenzfarbstoffe werden als Gemisch verwendet und müssen geeignete chemische und fluoreszenzspektroskopische Eigenschaften aufweisen. Die Berechnung der Konzentrationen aus den gemessenen Fluoreszenzintensitäten erfolgt unter Berücksichtigung und Korrektur mehrerer prozessbedingter Einflussgrößen. Aus den so gemessenen Konzentrationsfeldern wird das Feld des lokalen Deviationsgrades als quantitatives Maß für die Mikrovermischung berechnet.

Der Ablauf des Makro- und Mikromischens wird für Zelluloselösungen mit unterschiedlichen Massenanteilen und damit unterschiedlichen Viskositäten visualisiert. Für konstante Reynoldszahlen ist eine starke Abhängigkeit des Prozessfortschritts von der Viskosität sowie von der Position der Farbstoffzugabe zu beobachten. Die Mikrovermischung setzt zuerst in den Randbereichen der sich im Strömungsfeld ausbildenden Lamellen ein. Die Lamellendicke kann aus den Feldern des lokalen Deviationsgrades gemessen werden.

Zur Interpretation der gemessenen Konzentrationsfelder werden die Geschwindigkeitsfelder mit dem kommerziellen Programm CFX 4.4 berechnet. Der in den Experimenten zu beobachtende verschlechterte Ablauf der Vermischung mit zunehmender Viskosität lässt sich auf eine schwächer ausgeprägte Sekundärströmung im Rührbehälter zurückführen.

Stichworte: Mikromischen, Rührbehälter, Laserinduzierte Fluoreszenz (LIF) 


\section{Abstract \\ Kling, Kerstin \\ Visualisation of Micro- and Macromixing using two fluorescent and chemically reactive dyes}

For the progress of chemical reactions complete mixing of the reactants on molecular scale is required. Most techniques for the measurement of micromixing assume ideal macromixing. But during the laminar mixing process local concentrations strongly vary throughout the mixing vessel. Therefore, in this work mixing on macroscopic length scales as well as concentration differences on molecular scale are visualized simultaneously. For this purpose a mixture of two fluorescent dyes is injected into the vessel. The inert dye is a tracer for the macromixing. The fluorescence intensity of the reacting dye is enhanced by a chemical reaction. Since the reaction requires mixing on molecular scale the reacting dye indirectly visualizes the micromixing.

The concentration fields of both dyes are measured simultaneously with high spatial and temporal resolution using the Two-Colour-Laser Induced Fluorescence (LIF) technique. The fluorescent dyes are used as a mixture and must have appropriate chemical and spectroscopic properties. For the calculation of the concentrations form measured fluorescence intensities the influence of various process parameters is determined and corrected. From those concentrations the field of the local degree of deviation is finally calculated as a quantitative measure for the micromixing.

The course of the macro- and micromixing is visualized for cellulose solutions of different mass fractions and therefore different viscosity. For constant Reynolds numbers a strong influence of viscosity and the position of the dye injection on the progress of mixing is recognized. Micromixing mostly starts in the boundary layer of the lamellas which are created in the flow field. The striation thickness can be determined from the field of the local degree of deviation.

For the interpretation of the measured concentration fields the flow field is numerically calculated using the commercial software CFX 4.4. The reduced mixing performance which is measured for fluids with increasing viscosity is caused by a weaker secondary flow compared to the primary rotational flow.

Keywords: Micromixing, stirred vessel, Laser Induced Fluorescence (LIF) 


\section{Inhaltsverzeichnis}

Formelzeichen . . . . . . . . . . . . . . . . . . VI

1 Einleitung 1

2 Ziele der Arbeit $\quad 3$

3 Stand der Forschung 5

3.1 Mikro- und Makromischen . . . . . . . . . . . . . . . . 5

3.1.1 Mikromischmodelle . . . . . . . . . . . . . . . . . 5 5

3.1 .2 Bekannte Messverfahren . . . . . . . . . . . . . . . . . . . 9

3.2 Strömungsfelder und Energiedissipation in gerührten Behältern . . . . . . . 17

3.2.1 Numerische Ergebnisse . . . . . . . . . . . . . . . . . . 17

3.2.2 Experimentelle Ergebnisse . . . . . . . . . . . . . . . . . . . . 19

4 Visualisieren des Mikro- und Makromischens mit Hilfe von Tracern 22

5 Fluoreszenzspektroskopische Messungen $\quad 26$

5.1 Messungen mit dem Fluoreszenzspektrometer . . . . . . . . . . . . . . . . 28

5.2 Messungen mit dem Absorptionsspektrometer . . . . . . . . . . . . . . 32

5.3 Quantitative Auswertung . . . . . . . . . . . . . . . . 35

$6 \quad$ Eingesetzte Flüssigkeiten und Farbstoffe 38

6.1 Kriterien für die Auswahl des Farbstoffsystems . . . . . . . . . . . . . 38

6.2 Farbstoffe und Lösungsmittel . . . . . . . . . . . . . . . . . . . . 42

6.3 Versuchsdurchführung . . . . . . . . . . . . . . . . . . . . 49

7 Strömungsfelder im Rührgefäß 51

7.1 Rührgefäß und Rührer . . . . . . . . . . . . . . . . . . . 51

7.2 Transportgleichungen . . . . . . . . . . . . . . . . . . 51

7.3 Rheologische Fließgesetze . . . . . . . . . . . . . . . . 53

7.3.1 Newtonsche Fluide . . . . . . . . . . . . . . . . 54

7.3.2 Nicht-newtonsche Fluide . . . . . . . . . . . . . . . . 55

7.4 Rechengitter und Randbedingungen . . . . . . . . . . . . . . 56

7.5 Berechnete Geschwindigkeitsfelder . . . . . . . . . . . . . . . . . . 59 
8 Zwei-Farben Laserinduzierte Fluoreszenz $\quad 65$

8.1 Optischer Aufbau . . . . . . . . . . . . . . . . . . . 65

8.2 Kalibrierung des Messsystems . . . . . . . . . . . . . . . . 69

8.2 .1 Doppelbildoptik . . . . . . . . . . . . . . . . . . . 69

8.2.2 Messung der Energie gepulster Laser . . . . . . . . . . . . . . 71

8.2.3 Korrektur des Intensitätsprofils . . . . . . . . . . . . . 73

8.2.4 Fluoreszenzintensität und Konzentration . . . . . . . . . . . 75

8.3 Ablauf der quantitativen Auswertung . . . . . . . . . . . . . . . . 82

9 Ergebnisse der experimentellen Untersuchungen $\quad 85$

9.1 Messgenauigkeit . . . . . . . . . . . . . . . . . . 85

9.2 Einfluss des Zugabeortes . . . . . . . . . . . . . . . 86

9.3 Einfluss der Viskosität . . . . . . . . . . . . . . . . . . . . 91

9.4 Lamellendicke . . . . . . . . . . . . . . . . . . . . . . . . . . 94

$\begin{array}{ll}10 \text { Zusammenfassung } & 100\end{array}$

$\begin{array}{ll}\text { Literaturverzeichnis } & 102\end{array}$ 


\section{Formelzeichen}

\begin{tabular}{|c|c|c|}
\hline Symbol & Einheit & Bedeutung \\
\hline$A$ & - & Absorption \\
\hline$c$ & $\mathrm{~mol} / \mathrm{m}^{3}$ & Konzentration \\
\hline$C_{0}$ & - & Anfangskonzentrationsverhältnis \\
\hline$d_{r}$ & $\mathrm{~m}$ & Durchmesser des Rührorgans \\
\hline$D$ & $\mathrm{~m}$ & Durchmesser des Rührbehälters \\
\hline$D$ & $\mathrm{~m}^{2} / \mathrm{s}$ & Diffusionskoeffizient \\
\hline$\underline{\underline{D}}$ & $\mathrm{~s}^{-1}$ & Deformationsgeschwindigkeitstensor \\
\hline $\bar{e}$ & - & Eulersche Konstante \\
\hline $\bar{e}$ & $\mathrm{~W} / \mathrm{m}^{2}$ & Laserenergie Referenzwert, Gl. (8.2) \\
\hline$f^{I I}$ & - & Filterfaktor, Gl. (8.12) \\
\hline$g$ & $\mathrm{~m} / \mathrm{s}^{2}$ & Erdbeschleunigung \\
\hline$h$ & $\mathrm{~m}$ & Bodenabstand \\
\hline$i$ & $\mathrm{~W} / \mathrm{m}^{2}$ & Intensität \\
\hline$I$ & $\mathrm{~W} / \mathrm{m}^{2}$ & Integrale Intensität \\
\hline$I_{F}$ & $\mathrm{~W} / \mathrm{m}^{2}$ & Fluoreszenzintensität \\
\hline$I_{F 1}^{\prime}$ & $\mathrm{W} / \mathrm{m}^{2}$ & Fluoreszenzintensität von fluo-4 für Calcium-freie Lösung \\
\hline$I_{F 1}^{\prime \prime}$ & $\mathrm{W} / \mathrm{m}^{2}$ & Fluoreszenzintensität von fluo-4 im Sättigungszustand \\
\hline$k$ & $\mathrm{~m}^{3} / \mathrm{mol} \mathrm{s}$ & Reaktionsgeschwindigkeitskonstante \\
\hline$k_{M / O}$ & - & Metzner-Otto Konstante \\
\hline$K$ & - & Konsistenzfaktor \\
\hline$K$ & - & Parameter in Gl. (5.18) \\
\hline$l$ & $\mathrm{~m}$ & Durchstrahlungslänge \\
\hline$\underline{\underline{L}}$ & $\mathrm{~s}^{-1}$ & Geschwindigkeitsgradiententensor \\
\hline $\bar{M}$ & - & Korrekturmatrix, Gl. (8.3) \\
\hline$m$ & $\mathrm{~mol} / \mathrm{W} \mathrm{m}$ & Proportionalitätsfaktor, Gl. (5.19) \\
\hline$n$ & - & Fließexponent \\
\hline$n_{r}$ & $\mathrm{~s}^{-1}$ & Drehzahl \\
\hline$p$ & $\mathrm{~Pa}$ & Druck \\
\hline Res & - & Abbildungsmaßstab, Gl. (8.1) \\
\hline$s$ & $\mathrm{~m}$ & Strahlweg \\
\hline$s_{B}$ & $\mathrm{~m}$ & Abgebildeter Abstand \\
\hline$s_{G}$ & $\mathrm{~m}$ & Wahrer Abstand \\
\hline$s_{m}$ & $\mathrm{~m}$ & Mittlere Lamellendicke \\
\hline$t$ & $\mathrm{~s}$ & Zeit \\
\hline
\end{tabular}




\begin{tabular}{lll}
$V$ & $\mathrm{~m}^{3}$ & Volumen \\
$v$ & $\mathrm{~m} / \mathrm{s}$ & Geschwindigkeit \\
$\underline{\underline{W}}$ & $\mathrm{~s}^{-1}$ & Drehgeschwindigkeitstensor \\
$x, y, z$ & $\mathrm{~m}$ & Kartesische Koordinaten \\
$\alpha$ & $\mathrm{mol} / \mathrm{m}^{3}$ & Erstes zentrales Moment der Konzentrationsverteilung \\
$\beta$ & - & Verstärkungsfaktor, Gl. (6.13) \\
$\dot{\gamma}$ & $\mathrm{s}^{-1}$ & Scherrate \\
$\varepsilon$ & $\mathrm{m}^{2} / \mathrm{mol}$ & Molarer Extinktionskoeffizient \\
$\varepsilon^{\prime}$ & $\mathrm{m}^{2} / \mathrm{mol}$ & Molarer Absorptionskoeffizient \\
$\eta$ & $\mathrm{Pa} \mathrm{s}$ & Viskosität \\
$\eta_{0}$ & $\mathrm{~Pa} \mathrm{~s}$ & Anfangsviskosität \\
$\eta_{\infty}$ & $\mathrm{Pa} \mathrm{s}$ & Endviskosität \\
$\lambda$ & $\mathrm{m}$ & Wellenlänge \\
$\xi$ & & Massenanteil \\
$\rho$ & $\mathrm{kg} / \mathrm{m}^{3}$ & Dichte \\
$\sigma^{2}$ & $\left(\mathrm{~mol} / \mathrm{m}^{3}\right)^{2}$ & Zweites zentrales Moment der Konzentrationsverteilung \\
$\tau$ & - & Transmissionsgrad \\
$\underline{\tau}$ & $\mathrm{Pa}$ & Spannungstensor \\
$\psi$ & & Abbildungsfunktion \\
$\omega$ & - & Winkelgeschwindigkeit \\
$\Delta$ & & Deviationsgrad \\
& & Quanteneffizienz \\
\hline & &
\end{tabular}

\section{Indices}

\begin{tabular}{|c|c|}
\hline Symbol & Bedeutung \\
\hline 0 & Anfangszustand \\
\hline 1 & Reagierender Farbstoff \\
\hline 2 & Inerter Farbstoff \\
\hline$I$ & Filter I (BP 523) \\
\hline$I I$ & Filter II (RG 645) \\
\hline$a$ & absorbiert \\
\hline avg & gemittelt \\
\hline detekt & gemessen \\
\hline em & Emission \\
\hline$e x$ & Exzitation \\
\hline ges & gesamt \\
\hline
\end{tabular}




$\begin{array}{ll}\text { glob } & \text { global } \\ \text { korr } & \text { korrigiert } \\ \text { MFR } & \text { Multiple Frame of Reference } \\ \text { mol } & \text { Molekül } \\ n R & \text { nicht reagiert } \\ P & \text { Probe } \\ r & \text { Rührorgan } \\ R & \text { Reaktionsprodukt } \\ r e l & \text { normiert auf den Maximalwert } \\ r e p & \text { repräsentativ } \\ \text { sek } & \text { Sekundärströmung } \\ x, y, z & \text { in Richtung der kartesischen Koordinaten } \\ x, r, \phi & \text { in Richtung der zylindrischen Koordinaten }\end{array}$

\section{Kennzahlen}

$\underline{\text { Kennzahl }}$ Bedeutung

$R e \equiv \frac{n d_{r}^{2} \rho}{\eta} \quad$ Reynolds-Zahl

$S c \equiv \frac{\eta}{\rho D} \quad$ Schmidt-Zahl

\section{Abkürzungen im Text}

Abkürzung Bedeutung

CFD Computational Fluid Dynamics

CSV Cylindrical Stretched Vortex- Modell

DNS Direkte Numerische Simulation

EDD Engulfment Deformation Diffusion- Modell

GMM Generalized Mixing Model

IEM Interaction by Exchange with the Mean- Modell

LDA Laser Doppler Anemometrie

LES Large Eddy Simulation

LIF Laserinduzierte Fluoreszenz

PIV Particle Image Velocimetry

RANS Reynolds-Averaged-Navier-Stokes Gleichungen

PLIF Planare Laserinduzierte Fluoreszenz 


\section{Einleitung}

Mischvorgänge treten als für die Produktqualität und Verfahrensausbeute bestimmender Prozessschritt in zahlreichen Verfahren der chemischen, pharmazeutischen sowie der Lebensmittelindustrie auf. Je nach Beschaffenheit der zu mischenden Komponenten wird zwischen unterschiedlichen Mischaufgaben unterschieden:

- Homogenisieren: Verteilen von ineinander löslichen Flüssigkeiten

- Emulgieren: Verteilen von nicht mischbaren Flüssigkeiten

- Dispergieren: Verteilen eines Gases in einer Flüssigkeit

- Suspendieren: Verteilen eines partikelförmigen Feststoffs in einer Flüssigkeit

In dieser Arbeit wird das Homogenisieren von zwei ineinander löslichen Flüssigkeiten betrachtet. Alle Mischprozesse dienen dazu, lokal vorliegende Konzentrations- oder Temperaturgradienten zwischen den Komponenten der zu vermischenden Phasen auszugleichen. Dies geschieht auf makroskopischer Ebene durch Konvektion und auf molekularer Ebene infolge Diffusion.

Zum Mischen stehen verschiedene Apparate zur Verfügung. Neben den kontinuierlich betriebenen statischen Mischern werden sehr häufig Rührkessel eingesetzt, die sowohl kontinuierlich als auch diskontinuierlich betrieben werden. Dabei wird die Energie durch einen mechanisch bewegten Rührer in den Behälter eingetragen. Der Grund für die weite Verbreitung der Rührkesselapparate liegt in ihrem relativ einfachen, kompakten und damit kostengünstigen Aufbau und ihrer zugleich vielseitigen Verwendbarkeit. Eine Rührkesselanordnung kann durch geringfügige Änderungen, wie zum Beispiel dem Austausch des Rührers, auch für andere Mischaufgaben (s.o.) eingesetzt werden.

Meist wird aufgrund der ausgeprägten Quervermischung eine turbulente Strömung im Rührbehälter angestrebt. Viele chemische Einsatzstoffe besitzen aber eine so hohe Viskosität, dass sie im laminaren Strömungsbereich vermischt werden. Dazu zählen z.B. Produkte der Polymer- und Farbstoffindustrie. Der zur Turbulenzerzeugung benötigte Leistungseintrag wäre so hoch, dass die Prozesse unwirtschaftlich wären oder es aufgrund der hohen dissipierten Leistung zu einer thermischen Degradation der Produkte käme. In dieser Arbeit wird deshalb das Vermischen im laminaren Strömungsbereich untersucht. Mit den sich während des homogenen Vermischens von zwei ineinander löslichen Flüssigkeiten ablaufenden Strömungsvorgängen haben sich Villermaux [1], Baldyga und Pohorecki [2], Ottino [3] sowie Geisler, Mersmann und Voit [4] in experimentellen und theoreti- 
schen Arbeiten beschäftigt. Demzufolge müssen drei Teilschritte unterschieden werden, die sowohl konsekutiv als auch simultan stattfinden. Im ersten Teilschritt des Makromischens werden einzelne, durch ihre Konzentration gekennzeichnete Teilvolumen im gesamten Mischer durch konvektiven Transport verteilt. Lokale Konzentrationsschwankungen sowie die Ausdehnung der Teilvolumen bleiben dabei im Wesentlichen erhalten. Es findet lediglich eine Deformation infolge viskoser Reibung statt. Im zweiten Teilschritt des Makromischens werden die Abmessungen der Teilvolumen je nach Viskosität der Fluide entweder durch molekularen oder turbulenten Impulsaustausch reduziert. Dabei nimmt die Größe der durch eine homogene Konzentration charakterisierten Teilvolumen bis auf einen Grenzwert ab. Dieser kennzeichnet den Übergang von der Makro- zur Mikrovermischung. Unterhalb dieser Grenzgröße sind die Volumenelemente durch turbulente Schwankungsbewegungen nicht weiter zerteilbar. Der weitere Konzentrationsausgleich wird allein durch molekulare Diffusion verursacht.

Dem Makro- und Mikromischvorgang wird jeweils eine Zeitkonstante $\Theta_{\text {Makro }}$ bzw. $\Theta_{\text {Mikro }}$ zugeordnet, wobei der Teilschritt mit der größeren Zeitkonstante die Geschwindigkeit der Vermischung bestimmt. Sind die beschriebenen Vorgänge überlagert von homogenen chemischen Reaktionen zwischen zwei oder mehr Komponenten, so hängen die umgesetzten Reaktionsströme stark vom Fortschritt der Vermischung ab, wenn die Mikromischzeit von gleicher Größenordnung oder länger als die Zeitskala der chemischen Reaktion ist. Der Mischvorgang bestimmt dann im Wesentlichen die Geschwindigkeit des Reaktionsprozesses. Durch eine zu langsame Vermischung können unter Umständen unerwünschte Nebenprodukte entstehen, welche die Produktqualität negativ beeinflussen.

Häufig werden zur Charakterisierung des Mischfortschrittes im turbulenten oder laminaren Strömungsfeld nur zeitliche oder volumetrisch gemittelte Größen wie z.B. der Leistungseintrag oder die Mischzeit verwendet. Um den Ablauf einer chemischen Reaktionen verlässlich vorausberechnen zu können, müssen aber die zeitlich und lokal veränderlichen Konzentrationen auf den Mikroskalen des Ortsmaßstabes bekannt sein. Von großem technischen Interesse sind daher Verfahren zur ortsaufgelösten Visualisierung dieser Größen. 


\section{Ziele der Arbeit}

Der zeitliche Ablauf des laminaren Mikromischens wird lokal nach den Ortskoordinaten aufgelöst bisher nur selten untersucht. Insbesondere für laminare Mischvorgänge haben volumetrisch gemittelte Messwerte nur begrenzte Aussagefähigkeit, da der molekulare Transport nicht unabhängig vom (relativ langsamen) konvektiven Transport ist. Das gleichzeitige Beobachten des Makro- und Mikromischens ist deshalb notwendig, um neue Erkenntnisse über den Ablauf des laminaren Mischvorgangs zu erhalten.

Ziel der vorliegenden Arbeit ist es, sowohl die Vermischung auf makroskopischen Längenskalen als auch die Zusammensetzung von fluiden Gemischen im Skalenbereich molekularer Abmessungen zu visualisieren. Dazu wird eine optische Messtechnik mit hoher örtlicher und zeitlicher Aufösung, die Zwei-Farben Laserinduzierte Fluoreszenz, eingesetzt. Die Konzentrationsfelder eines inerten Fluoreszenzfarbstoffs und eines auf einer anderen Wellenlänge fluoreszierenden Reaktionsprodukts können so simultan gemessen werden. Die Verteilung des inerten Farbstoffs wird im Rührgefäß durch den konvektiven Transport bestimmt. Die Bildung des Reaktionsprodukts setzt die molekulare Vermischung voraus und dient als indirekter Nachweis für die molekulare Vermischung.

Kapitel 3 dieser Arbeit gibt einen Überblick über den Stand der Forschung sowie theoretische und experimentelle Arbeiten zum Mikro- und Makromischen. Ein quantitatives Maß für die Mikrovermischung wird in Kapitel 4 in Form des lokalen Deviationsgrades hergeleitet. Der lokale Deviationsgrad wird aus den gemessenen Konzentrationen des inerten Farbstoffs und des Reaktionsprodukts berechnet und gibt den Anteil des reagierenden Farbstoffs an, der lokal noch nicht umgesetzt ist. In Kapitel 5 werden die Grundlagen der quantitativen Fluoreszenzspektroskopie erläutert und in Kapitel 6 ein geeignetes Stoffsystem aus Fluoreszenzfarbstoffen und Lösungsmitteln ausgewählt. Anschließend wird der Aufbau und die Kalibrierung des verwendeten Messsystem beschrieben. Die Berechnung der Konzentrationen aus den gemessenen Fluoreszenzintensitäten erfolgt unter Berücksichtigung und Korrektur mehrerer prozessbedingter Einflussgrößen (Kapitel 8).

In Kapitel 9 wird der Ablauf des Makro- und Mikromischens für wässrige Zelluloselösungen mit unterschiedlichen Massenanteilen und damit unterschiedlichen Viskositäten visualisiert und gemessen. Dabei wird der Einfluss der Viskosität und der Position der Farbstoffzugabe untersucht. Um die Zugabepositionen für die Farbstoffzugabe während der experimentellen Untersuchungen auszuwählen und die experimentellen Ergebnisse zu 
interpretieren, werden die Geschwindigkeitsfelder und lokalen Deformationsgeschwindigkeiten im Rührbehälter mit einem kommerziellen Programm zur Strömungssimulation berechnet (Kapitel 7). 


\section{Stand der Forschung}

\subsection{Mikro- und Makromischen}

In Kapitel 1 ist der prinzipielle Ablauf des Makro- und Mikromischens zweier ineinander löslicher Flüssigkeiten dargestellt. Als Erster hat Danckwerts [5] ein Gütemaß für die Mikro- und Makromischung vorgeschlagen. Der von ihm definierte Längenmaßstab der Segregation beschreibt die Abmessungen von Fluidelementen mit konstanter Konzentration $c^{i}$. Die Konzentrationsunterschiede zwischen den einzelnen Fluidelementen $i$ werden mit der Intensität der Segregation beschrieben

$$
I_{s}(t)=\frac{\sigma^{2}(t)}{\sigma_{0}^{2}}=\frac{\sum_{i}\left(c^{i}(t)-\bar{c}\right)^{2}}{\sum_{i}\left(c_{0}^{i}-\bar{c}\right)^{2}} .
$$

Diese ist definiert als Quotient aus der Varianz $\sigma^{2}(t)$ der Konzentrationsverteilung $c(t)$ bezogen auf die Varianz $\sigma_{0}^{2}$ zum Anfangszeitpunkt. Die unmittelbare Bestimmung des Längenmaßstabs und der Intensität der Segregation ist schwierig, da Messungen oder numerische Berechnungen eine Ortsauflösung im molekularen Maßstab erfordern. In den beiden folgenden Kapiteln werden Modelle für die Berechnung des Mikromischprozesses sowie erprobte Messverfahren vorgestellt, die die Güte der Vermischung indirekt zu messen gestatten.

\subsubsection{Mikromischmodelle}

Die Berechnung der volumetrischen Durchmischung diskreter Fluidelemente ist mit Hilfe kommerziell verfügbarer Programmsysteme, besonders durch die immer größer werdenden Computerleistungen, möglich. Die Transportvorgänge im Mikromaßstab der Ortskoordinaten können jedoch wegen einer begrenzten örtlichen Aufösung der mathematischen Gitter auch heute noch nur für einfach berandete Strömungsfelder und relativ kleine Re-Zahlen berechnet werden. Dabei wird die Methode der Direkten Numerischen Simulation (DNS) angewendet, die alle notwendigen Transportgleichungen löst (z.B. Gerlinger, Schneider und Bockhorn [6, 7]). Für komplexere Strömungsfelder muss auf Modelle zurückgegriffen werden, die den Stoff- und Energietransport im Ortsbereich des Mikromaßstabs beschreiben. Dabei existieren zwei unterschiedliche Methoden. Der Mischvorgang kann ähnlich der Modelle zum turbulenten Transport auf statistischem Wege be- 


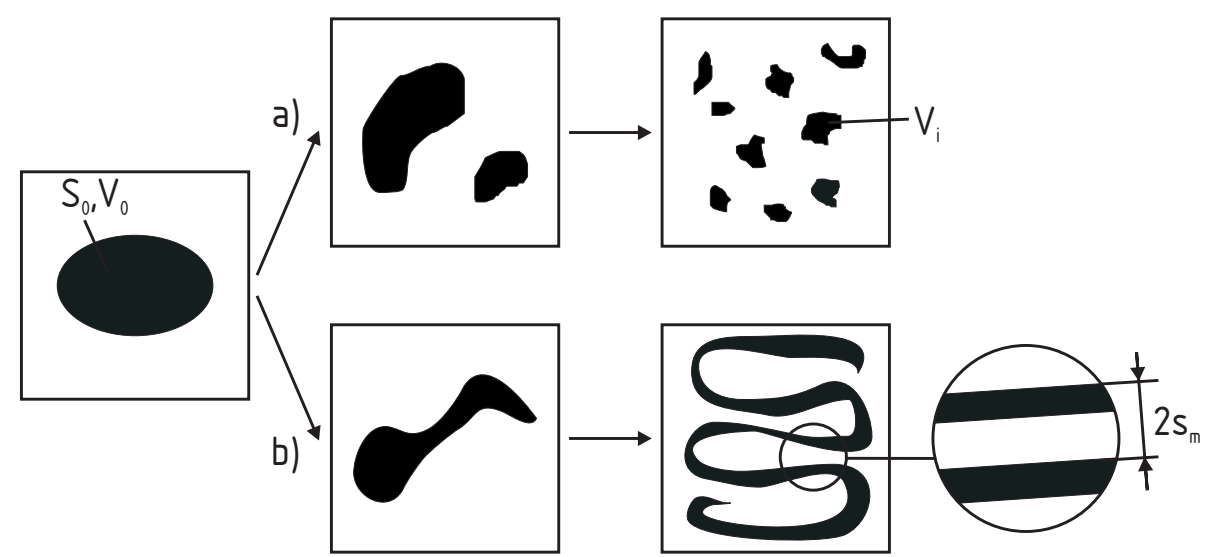

Bild 3.1: Schematischer Ablauf a) des turbulenten und b) des laminaren Mischvorgangs

schrieben werden. Eine andere Methode beschreibt das Mikromischen auf kleinen Ortsskalen in deterministischer Weise, während für das Vermischen auf großen Ortsskalen vollständige oder teilweise Makrovermischung angenommen wird. Viele Modelle liefern nur zeitabhängige Ergebnisse für die globale Änderung der Spezieskonzentrationen, nicht aber lokal aufgelöste Konzentrationsfelder. Im Folgenden wird ein Überblick gegeben, der jedoch keinen Anspruch auf Vollständigkeit erhebt.

\section{Turbulente Mikromischmodelle}

Eine Übersicht über die heute zur Verfügung stehenden Modelle geben Fox [8], Baldyga und Bourne [9] sowie Bakker [10]. Die meisten Modelle gelten für turbulente Strömungsfelder und modellieren die Dissipation der turbulenten kinetischen Energie. Dabei wird vom Zerfall und der Deformation turbulenter Wirbel ausgegangen, wie er in Bild 3.1 a) schematisch dargestellt ist. Turbulente Schwankungsbewegungen bewirken, dass die Teilvolumen unvermischter Fluidelemente weiter zerteilt und somit ihre Ausdehnung verkleinert werden. Je größer die turbulenten Schwankungsgeschwindigkeiten sind, je größer also die Dissipationsrate der turbulenten kinetischen Energie ist, desto kleiner ist die resultierende Ausdehnung der Teilvolumen $V_{i}$.

Das von Bourne und Baldyga [11] vorgeschlagene Engulfment-Deformation-Diffusion (EDD)- Modell beschreibt die Wirbelstrukturen als sich aufrollende dünne Schichten unterschiedlicher Zusammensetzung. Für den Mikromaßstab der Ortskoordinate wird die Diffusion und die chemische Reaktion mit Hilfe einer eindimensional formulierten Massenbilanz beschrieben. Die Wirbel werden deformiert und gestreckt, wobei die Deformationsgeschwindigkeit vom Grad der Turbulenz abhängt. Für kleine Schmidt-Zahlen (Sc « 4000) kann angenommen werden, dass Deformation und Diffusion viel schneller ablaufen als das Aufrollen der Wirbel. In diesen Fällen führt auch das vereinfachte, 0-dimensionale Engulfment-Modell zu guten Ergebnissen [12]. Eine Erweiterung des EDD- 
Modells von Bourne [13] berücksichtigt, dass infolge unvollständiger Makrovermischung nicht das gesamte Behältervolumen für die Mikrovermischung zur Verfügung steht. Für den mesovermischten Volumenanteil wird deshalb eine zusätzliche Bilanzgleichung gelöst. Auf der Grundlage von Ergebnissen über verwirbelte Strömungen mit Direkter Numerischer Simulation entwickeln Bakker und van den Akker [14, 15] das Cylindrical-StretchedVortex (CSV)-Modell. Die Streckung der kleinsten Wirbelstrukturen im viskosen Unterbereich wird beim Lösen einer zweidimensionalen Massenbilanz berücksichtigt.

Villermaux, David und Gouye [1] entwickeln ein Modell, welches sie mit Interaction by Exchange with the Mean (IEM)-Modell bezeichnen. Es berücksichtigt den Konzentrationsausgleich zwischen den Fluidelementen mit Hilfe einer Zeitfunktion. Der Ausgleich durch den molekularen Transport erfolgt um so schneller, je kleiner die geometrischen Abmessungen der Elemente und um so größer die Kontaktflächen zwischen den Elementen sind. Die volumetrische Ausdehnung der Fluidelemente wird von der örtlichen Turbulenz bestimmt. Mit einer weiteren Modellvorstellung, die Villermaux und Falk [16] als Generalized-Mixing-Model (GMM) bezeichnen, werden die Teilschritte des turbulenten Mischens durch unterschiedliche Zeitfunktionen beschrieben. Während Baldyga und Bourne diese mit Hilfe der Turbulenztheorie auf physikalischer Grundlage herleiten, muss das GMM-Modell als phänomenologischer Ansatz gelten.

\section{Laminare Mikromischmodelle}

Die Beschreibung der laminaren Vermischung ist ohne statistische Modelle möglich und kann deterministisch aus der Kinematik des Strömungsfeldes erfolgen [17]. In Bild 3.1 b) ist der laminare Mischvorgang schematisch dargestellt. Im Gegensatz zur tubulenten Vermischung bleibt das Volumen der Fluidaggregate konstanter Konzentration zunächst unverändert. Teilvolumen mit einer ursprünglichen Ausdehnung $s_{0}$ werden infolge viskoser Reibung lediglich gestreckt und gefaltet. So entsteht eine lamellenartige Struktur, die durch eine mittlere Lamellendicke $s_{m}$ gekennzeichnet ist. Mit zunehmender Verweilzeit in der Scher- bzw. Dehnströmung nimmt die mittlere Lamellendicke ab. Ottino, Ranz und Macosko [18] geben für einfache Geschwindigkeitsfelder Gleichungen für die zeitliche Veränderung der Lamellendicke in Abhängigkeit von der örtlichen Scherrate an. Je nach Ausrichtung der Lamellen zur Scherrichtung ergibt sich eine unterschiedlich starke Abnahme der Lamellendicke [17]. Für inkompressible, newtonsche Fluide wird eine obere Begrenzung für die Größe der erzeugten Grenzfläche formuliert, die von der viskosen Reibung abhängig ist.

Clifford [19] berechnet die Produktverteilung für eine Konsekutivreaktion zwischen Reaktanden in benachbarten Lamellen. Durch die Annahme einer gaußschen Normalverteilung 
für die Konzentrationsprofile in den Lamellen benötigt sein numerisches Modell nur kurze Rechenzeiten. Allerdings bleibt in dem Modell die Verformung der Lamellen durch das Strömungsfeld unberücksichtigt.

Das von Buchmann angegebene Modell für das laminare Mischen [20] berücksichtigt sowohl die Verformung von Fluidelementen als auch den davon abhängigen molekularen Stofftransport und die Reaktion. Dazu werden geeignete Transportgleichungen für die Lamellendicke und ein Mikromischgütemaß formuliert. Diese Gleichungen werden in ein kommerzielles Simulationsprogramm, das sowohl Geschwindigkeits- als auch Konzentrationsfelder auf numerischem Wege zu ermitteln gestattet, implementiert und ermöglichen so die Berechnung der Mikromischung. Aufgrund der raumfesten Formulierung der Transportgleichungen nach Euler liefert das Modell bisher nur für kurze Mischzeiten gute Ergebnisse.

Lamberto, Alvarez und Muzzio [21] berücksichtigen die Abhängigkeit von der Verweilzeit im Rührbehälter und verfolgen eine große Anzahl von Fluidelementen im materiellen Bezugssystem nach Lagrange. Dabei berechnen sie, welcher Dehnung die Fluidelemente auf ihrem Weg durch den Rührbehälter im Strömungsfeld ausgesetzt sind. Beispielhaft wird ein Rührbehälter mit 6-Blatt Scheibenrührer untersucht. Die Autoren stellen fest, dass Fluidelemente in den isolierten Mischzonen der Ringwirbel nur einer linear mit der Zeit ansteigenden Dehnrate ausgesetzt sind, während Fluidelemente in größerer Entfernung zum Rührer eine exponentiell ansteigende Dehnrate erfahren. Letzteres kann als Hinweis auf chaotische Strömungen gedeutet werden. Aufbauend auf [21] berechnen Zalc, Szalai, Alvarez und Muzzio [22] die Verformung von kleinen Fluidelementen in der Strömung eines Rührbehälters, der mit einem dreistufigen Scheibenrührer ausgestattet ist. Die Reorientierung der Fluidelemente durch die Rührerblätter kann so berechnet werden.

Den Einfluss der konvektiven und diffusiven Transportströme auf den Ablauf einer schnellen chemischen Reaktion haben Szalai, Kukura, Arratia und Muzzio [23] erst vor kurzem sowohl theoretisch also auch numerisch untersucht. Dabei kommt die Direkte Numerische Simulation für ein einfaches eindimensionales Lamellenmodell und für eine zweidimensionale periodische Strömung einfacher Geometrie zur Anwendung. Das Deformationsfeld hat einen entscheidenden Einfluss auf den Reaktionsprozess, wenn die Zeitkonstanten von Konvektion, Diffusion und chemischer Reaktion von gleicher Größenordnung sind. Die Wahrscheinlichkeitsdichtefunktion der Konzentration des Reaktionsprodukts weist über der Zeit einen selbstähnlichen Verlauf auf, wenn sie mit der mittleren Konzentration und der Standardabweichung normiert wird. Gleiche Tendenzen finden die Autoren für die Vermischung von reaktiven Komponenten in dem komplexen Strömungsfeld eines Rührbehälters. Dabei liegen experimentelle Daten für eine Säure-Base-Reaktion zugrunde. Die Produktverteilung wird mit Hilfe der Laserinduzierten Fluoreszenz gemessen und durch 
Anwendung bildverarbeitender Methoden ausgewertet. Numerisch berechnete Konzentrationsfelder eines inerten Tracers [24] zeigen geometrisch ähnliche Lamellenstrukturen und somit qualitativ eine brauchbare Übereinstimmung mit den experimentellen Daten. Aus dem Transport der Fluidelemente wird aber lediglich eine Kontaktfläche berechnet. Die chemische Reaktion der Spezies wird jedoch nicht berücksichtigt.

Der Zusammenhang zwischen Strömungsfeld, örtlicher Mischgüte und chemischer Reaktion ist für komplexe Geometrien zur Zeit noch nicht vollständig berechenbar.

\subsubsection{Bekannte Messverfahren}

\section{Mischzeiten}

In der Praxis sind die Parameter Leistung, Drehfrequenz und Mischzeit Grundlage für die Auslegung eines Rührwerks zum Homogenisieren von Flüssigkeiten. Makroskopische Mischzeiten können sehr einfach mit Hilfe von Sonden gemessen werden, die den zeitlichen Verlauf der Konzentrationen lokal anzeigen. Wenn die gemessenen zeitlichen Schwankungen der Konzentration unter einen festgelegten Wert abgeklungen sind, gilt der Mischvorgang als abgeschlossen. Allerdings hängt die gemessene Mischzeit von der Art und dem Ort der Tracerzugabe, der Anzahl und Position der Sonden, dem Probenvolumen der Sonden und dem Mischkriterium ab [25].

Viele Forschergruppen setzen Anfärbe- und Entfärbungsmethoden ein, welche die Verteilung eines Farbstoffes im Mischer visuell oder photoelektronisch zu messen gestatten. Mann, Pillai, El-Hamouz, Ying, Togatorp und Edwards [26] dosieren einen Farbstoff in den Mischer und vergleichen die Farbstoffverteilung mit berechneten Farbskalen. Mit dieser Anfärbemethode wird die makroskopische Vermischung gemessen, denn die lichtelektrische oder visuelle Messung von Farbstoffverteilungen kann Inhomogenitäten unterhalb der Größenordnung von Millimetern nicht aufösen. Die Entfärbungsmethode, bei der ein zunächst homogen verteilter Farbstoff durch eine Reaktion abgebaut wird, gestattet hingegen Aussagen über die mikroskopische Vermischung, da der Ablauf der chemischen Reaktion die molekulare Vermischung voraussetzt [27], [28]. Allerdings kann aus der völligen Entfärbung nicht auf eine vollständige Mikrovermischung geschlossen werden, wenn das Entfärbungsmittel im Überschuss zugegeben wird. Die mit unterschiedlichen Verfahren gemessenen Mischzeiten weichen daher für gleiche Versuchsbedingungen stark voneinander ab und können nicht miteinander verglichen werden [29].

Muzzio und Mitarbeiter [30] verwenden eine Farbumschlagmethode, um die Größe und Position strömungsarmer Zonen, sogenannter isolierter Mischzonen, zu visualisieren. Diese variieren in Abhängigkeit vom Rührorgan und der Drehzahl und bilden sich insbe- 
sondere für radial fördernde Rührer im laminaren Strömungsbereich aus. Mit Hilfe eines pH-Indikators und der Zugabe einer Säure in die vorgelegte basische Indikatorlösung werden diese isolierten Mischzonen blau sichtbar gemacht, während die mikrovermischten Bereiche eine gelbliche Färbung annehmen. Durch Anwendung eines zeitveränderlichen Drehzahlprotokolls werden die isolierten Mischzonen örtlich in ihrer Lage verschoben und die Mischung so erheblich verbessert [31]. Ascanio, Foucault und Tanguy [32] verwenden eine sehr ähnliche Methode zum Visualisieren isolierte Mischzonen beim Vermischen nicht-newtonscher Fluide. Ihnen gelingt es, den Stofftransport durch eine exzentrische Anordnung von ein oder zwei Rührorganen im Behälter zu verbessern.

Eine experimentell erprobte Methode zum Messen von Mikromischzeiten ist der Einsatz reagierender Stoffsysteme beim Mischen. Wenn zwischen den Edukten zwei miteinander konkurrierende chemische Reaktionen ablaufen, so lässt sich aus den gemessenen Stoffmengen der Produkte die Mikromischzeit berechnen. Bourne und Mitarbeiter [33] waren die ersten, die eine konkurrierende Konsekutiv-Reaktion eingesetzt haben, mit der zahlreiche Mikromischmodelle (s. Kapitel 3.1.1) überprüft werden konnten. Dabei reagieren zwei Reaktanden spontan zu einem Reaktionsprodukt R, welches in einer zweiten, langsameren Reaktion mit einem der Reaktanden ein anderes Reaktionsprodukt S bilden kann. Das Reaktionsprodukt S entsteht nur, wenn sich die Reaktanden langsam vermischen. Das Produktverhältnis R/S ist somit ein Maß für das Verhältnis von Mikromischzeit zur Reaktionszeit der Folgereaktion. Dieses Reaktionssystem setzt Bourne für viele Messungen in verschiedenen Rührgefäßen ein. Eine Ergebniszusammenfassung der zahlreichen Veröffentlichungen hierzu gibt Yu [34]. Andere konkurrierende Reaktionsysteme wurden unter anderem von Villermaux und Mitarbeitern [35] sowie von Assirelli, Bujalski, Nienow und Eaglesham [36] vorgeschlagen.

Mikromischzeiten können alternativ mit Hilfe konkurrierender Parallelreaktionen gemessen werden. Einen Überblick über erprobte Reaktionssysteme geben Baldyga und Bourne [37] sowie Yu [34]. Dabei reagiert der in limitierender Konzentration zugegebene Reaktand spontan zu einem Produkt R und schnell zu einem Produkt S. Im Falle idealer Mikrovermischung würde das Produktverhältnis R/S dem Verhältnis der Reaktionszeitkonstanten entsprechen und es würde fast nur $\mathrm{R}$ entstehen. In Bereichen nicht idealer Mikrovermischung entsteht auch das Produkt S, da durch die unvollständige Vermischung kein Produkt R gebildet werden kann. Das Produktverhältnis R/S ermöglicht Rückschlüsse auf das Verhältnis der Mikromischzeit zu der bekannten Reaktionszeit der schnellen Reaktion. Parallele Reaktionssysteme werden von Bourne, Hilber und Petrozzi [38], Rousseaux, Falk, Muhr und Plasari [39] sowie Fournier, Falk und Villermaux [40, 41] zur Charakterisierung der Vermischung in Rührbehältern, Rohrreaktoren, etc. verwendet.

Die Messmethode mit konkurrierenden Reaktionen setzt die ideale Makrovermischung 
der Komponenten voraus. Dieses wird durch sehr gering dosierte Mengen an Edukten erreicht, so dass an der Einspeisung immer genügende Mengen an unverbrauchtem Reaktorinhalt zur Verfügung stehen. Durch das Wandern des reagierenden Teilvolumens im Reaktor ist nur eine begrenzte lokale Zuordnung der gemessenen Mikromischzeiten möglich, da die Reaktion in Bereichen mit unterschiedlichen Mikromischzeiten abläuft. Für laminare Mischvorgänge kann diese Messmethode daher nicht eingesetzt werden, da dann die Mikrovermischung so langsam abläuft, dass die Reaktanden während der Mikrovermischung durch große Bereiche des Rührgefäßes transportiert werden. Es sind daher keine Aussagen über die örtlichen Mikromischgüten möglich. Um genaue Kenntnisse über den zeitlichen Ablauf der Vermischung in turbulenten oder laminaren Strömungsfeldern zu gewinnen, reicht es deshalb nicht aus, zeitliche oder volumetrisch gemittelte Größen zu messen. Vielmehr müssen die zeitlich und lokal veränderlichen Konzentrationen bekannt sein.

\section{Messen von Konzentrationsfeldern und abgeleiteter Größen}

Optische und tomographische Messmethoden besitzen zwei Vorteile gegenüber konventionellen Methoden. Erstens erfolgt die Messung berührungslos und damit ohne Rückwirkung auf den Prozess. Zweitens detektieren tomographische Methoden die gemessene Größe zeitgleich an allen Orten des Messraums. Daher sind auch instationäre Vorgänge gut zu beobachten, was mit einer zeitlichen Folge von lokalen Messungen nicht möglich ist. Einen umfassenden Überblick über derzeit zur Verfügung stehende optische und tomographische Messverfahren geben Mayinger und Feldmann [42].

Holden, Wang, Mann, Dickin und Edwards [43] wenden die elektrische Widerstandstomographie an und messen in einem Tank mit $3 \mathrm{~m}^{3}$ Inhalt das Vermischen einer zudosierten Salzlösung. Die optische Tomographie wird von Haarde [44, 45] und Renz [46, 47] eingesetzt, um die Ausbreitung von Notstoppern in Lagertanks zu messen. Buchmann und Mewes [48, 20] verwenden die tomographische Zweiwellenlängenphotometrie, um die dreidimensionalen Konzentrationsfelder eines inerten und eines reagierenden Farbstoffs simultan im gesamten Rührbehälter zu messen. Die Makro- und Mikromischung wird für Rührbehälter, die mit unterschiedlichen Rührorganen ausgestattet sind, vermessen. Die örtliche Auflösung ist aber nicht ausreichend, um die bei der Mischung entstehenden lamellenartigen Strukturen der Farbstoffschichten, also den Längenmaßstab der Segregation, aufzulösen.

Eine deutlich höhere örtliche Auflösung bei gleichzeitig hoher zeitlicher Auflösung kann mit Hilfe der Laserinduzierten Fluoreszenz (LIF) erreicht werden. Konzentrationen von fluoreszierenden Farbstoffen werden dabei entweder in einem Punkt, entlang einer Linie 
oder in einer Ebene gemessen. Bei Letzterem spricht man von Planarer Laserinduzierter Fluoreszenz (PLIF), die auch in dieser Arbeit zur Anwendung kommt. Die örtliche Auflösung ist von der verwendeten Abbildungsoptik abhängig und erlaubt prinzipiell das Detektieren kleinster Strukturen bis zur Kolmogorov-Länge. Somit kann die Laserinduzierte Fluoreszenz experimentelle Ergebnisse liefern, die mit der örtlichen Auflösung von mit der Direkten Numerischen Simulation (DNS) berechneten Ergebnissen vergleichbar sind und zu deren Überprüfung verwendet werden [15]. Allerdings können dreidimensionale Felder nur durch zeitlich getrennte Messungen rekonstruiert werden. Für kleine Messvolumen kann die Ebene des Lichtschnitts z.B. mit Hilfe eines rotierenden Spiegels sehr schnell durch das Volumen bewegt werden, so dass ein quasi- dreidimensionales Konzentrationsfeld gemessen wird [49]. Deusch und Dracos [50] prüfen unterschiedliche Scanverfahren auf ihre Eignung und schlagen die Kombination aus einem piezo-elektrischen Spiegel und einer Zylinderlinse für ein optimales Signal-zu-Rausch-Verhältnis vor. Für große konvektive Transportströme in Scanrichtung ist der zeitliche Versatz der Aufnahmen nicht mehr vernachlässigbar und eine Korrektur der Messwerte muss durchgeführt werden [51]. Ist die örtliche und zeitliche Aufösung groß genug, so kann aus dem dreidimensionalen Konzentrationsfeld das Vektorfeld der Konzentrationsgradienten durch direktes Differenzieren exrahiert werden. Durch exaktes Lösen der Differentialgleichung für den Speziestransport, die sowohl diffusive als auch konvektive Terme enthält, kann das Geschwindigkeitsfeld berechnet werden $[52,50]$. Die örtliche Auflösung ist dabei deutlich höher als mit anderen Lichtschnittverfahren zum Messen von Geschwindigkeiten, z.B. Particle Image Velocimetry (PIV) (s. Kapitel 3.2.2), erreichbar wäre.

Eine Liste einiger wichtiger Forschungsarbeiten, in denen Mischvorgänge mit Hilfe der Laserinduzierten Fluoreszenz untersucht wurden, sind in Tabelle 3.1 und Tabelle 3.2 zusammengestellt. Die meisten Autoren verwenden inerte Tracer, um das Makromischen für unterschiedliche Applikationen zu visualisieren (Unger und Muzzio [63], Houcine et al. [62, 61]). Arcoumanis, McGuirk, und Palm [77] überprüfen drei gebräuchliche Fluoreszenzfarbstoffe auf ihre Eignung zum Messen von Konzentrationsfeldern. Walker [78] untersucht die Abhängigkeit der Fluoreszenzintensität des weit verbreiteten Farbstoffs Fluorescein von den Parametern Temperatur, pH-Wert, Konzentration und Laserleistung und gibt an, unter welchen Voraussetzungen eine quantitative Auswertung möglich ist.

André, David und Villermaux [55] messen Konzentrationsschwankungen eines kontinuierlich in den Behälter dosierten Fluoreszenzfarbstoffs nacheinander an unterschiedlichen diskreten Orten im Behälter. Die Messfrequenz beträgt maximal 5kHz. Aus der Varianz der Konzentrationsschwankungen berechnen sie einen Segregationsindex. Distelhoff und Marquis [57, 56, 58] führen ebenfalls Messungen für kontinuierlich betriebene Rührbehälter durch. Während einer einzigen Messung kann aber die Konzentration entlang 
- $13-$

\begin{tabular}{|c|c|c|c|c|c|c|c|c|c|c|c|c|}
\hline 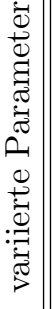 & & 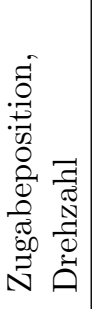 & 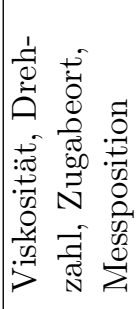 & & 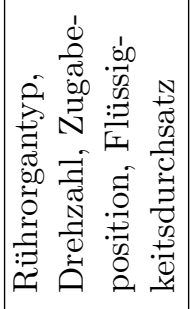 & & 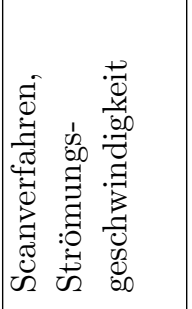 & & 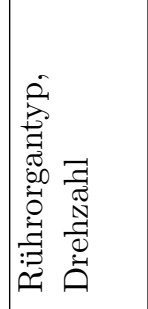 & 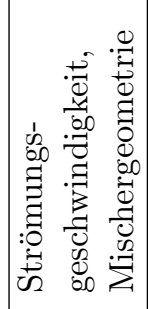 & & \\
\hline 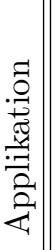 & & 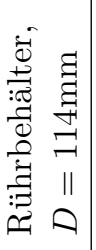 & 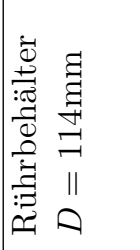 & & 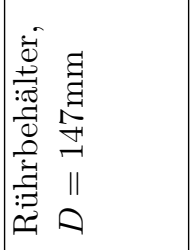 & 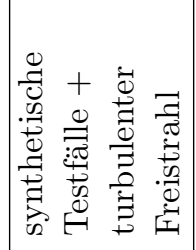 & 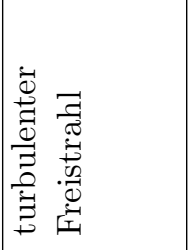 & & 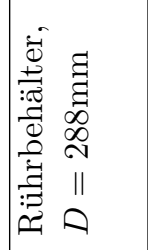 & 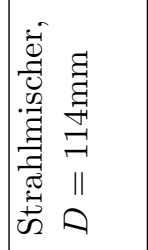 & 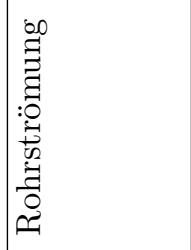 & 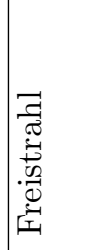 \\
\hline 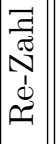 & & 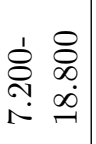 & & & 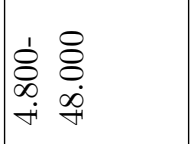 & $\begin{array}{l}8 \\
0 \\
0 \\
0\end{array}$ & 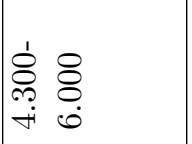 & & 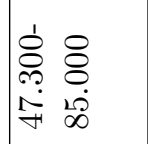 & 总 \& & $\underset{+}{\stackrel{8}{8}}$ & 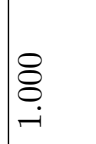 \\
\hline 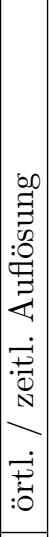 & & 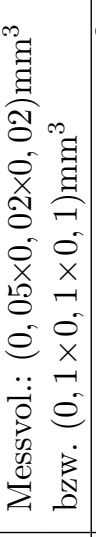 & 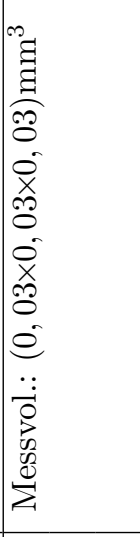 & & 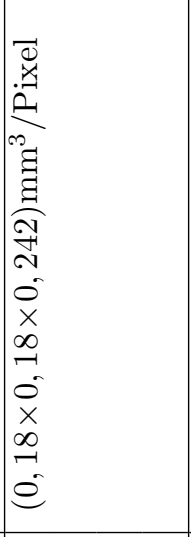 & 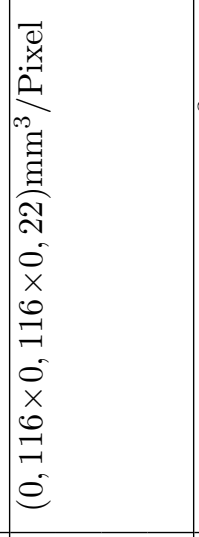 & 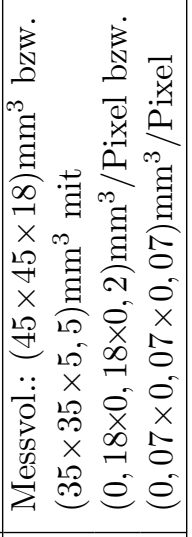 & & 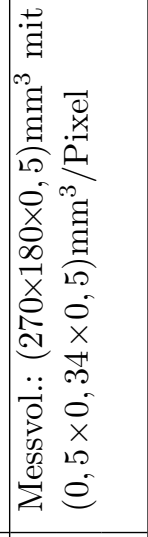 & 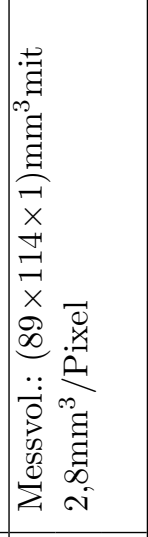 & 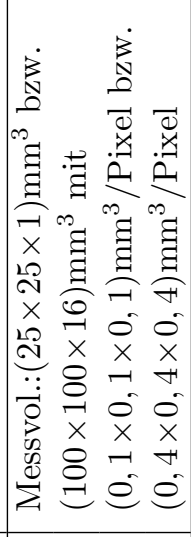 & 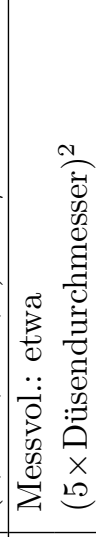 \\
\hline 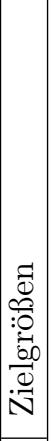 & & 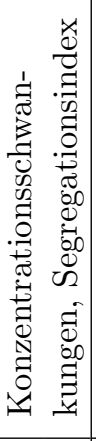 & 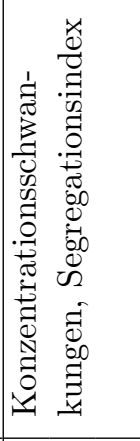 & & 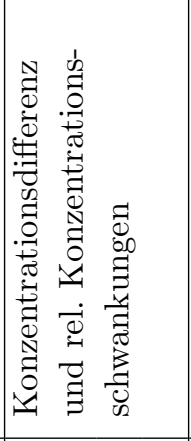 & 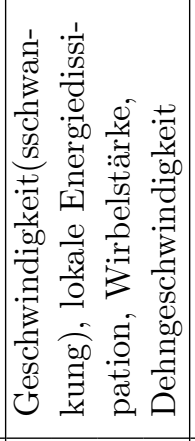 & 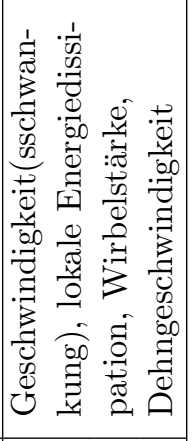 & & 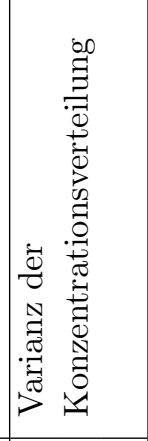 & 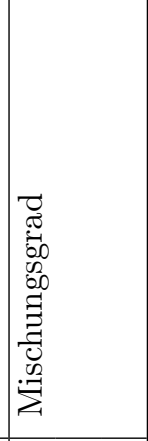 & 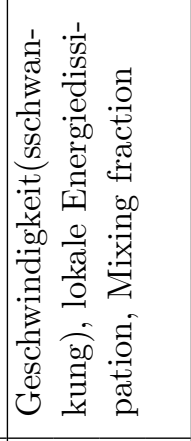 & 蒂 \\
\hline 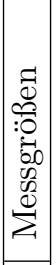 & & 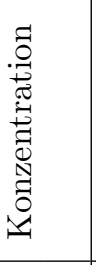 & 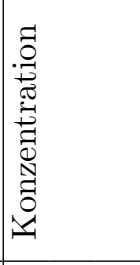 & & 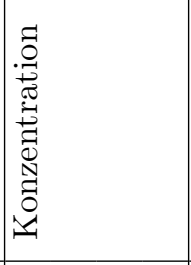 & 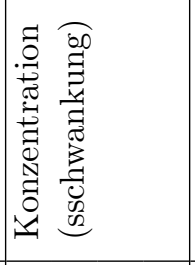 & 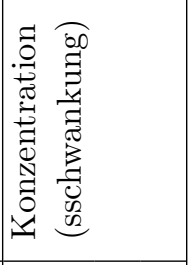 & & 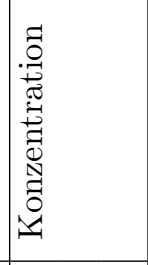 & 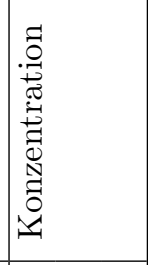 & 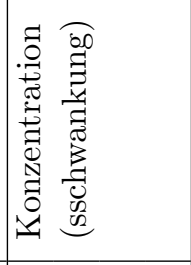 & 茪 \\
\hline 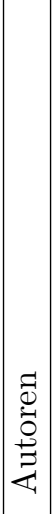 & 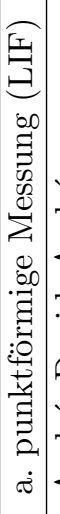 & 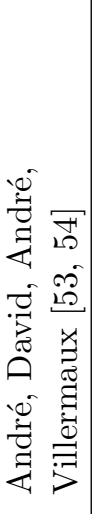 & 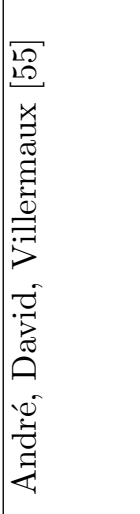 & 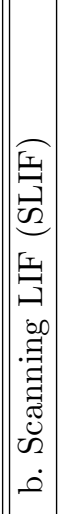 & 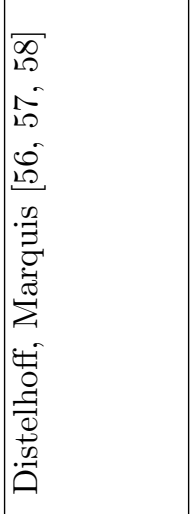 & 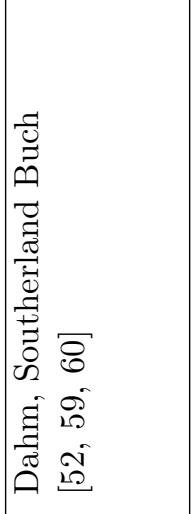 & 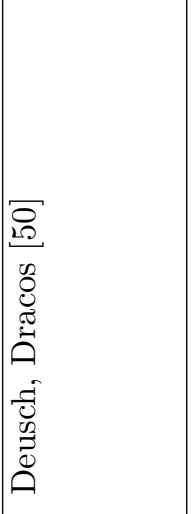 & 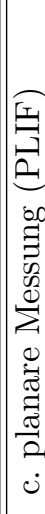 & 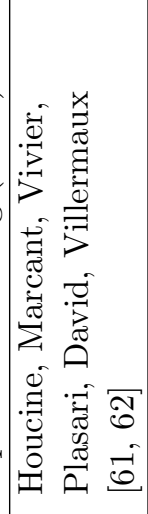 & 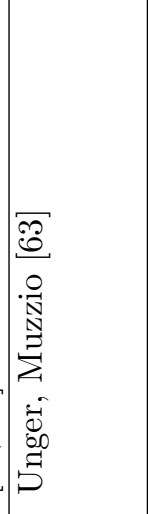 & 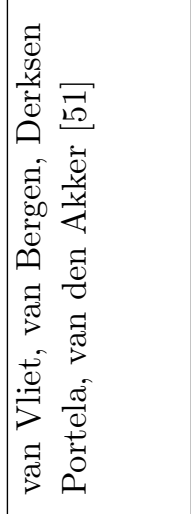 & 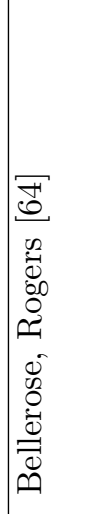 \\
\hline
\end{tabular}


$-14-$

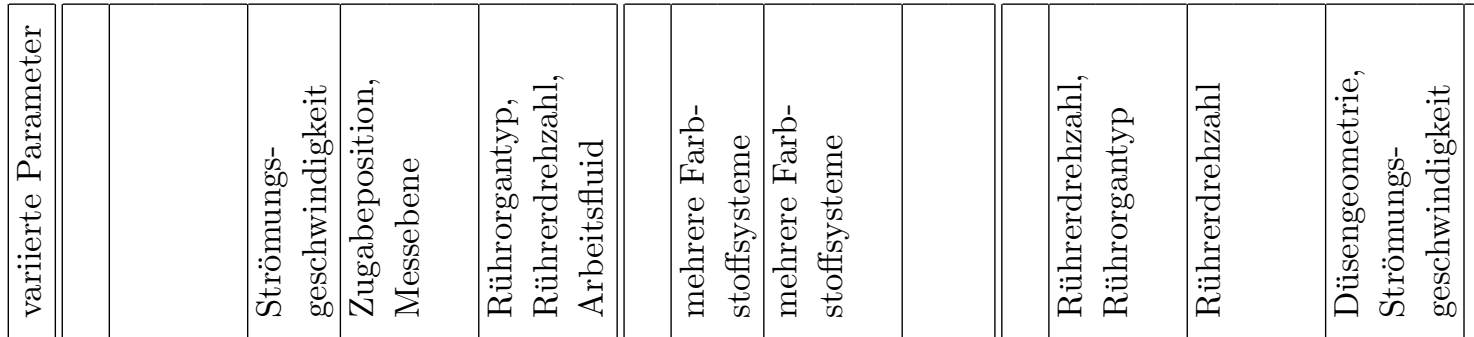

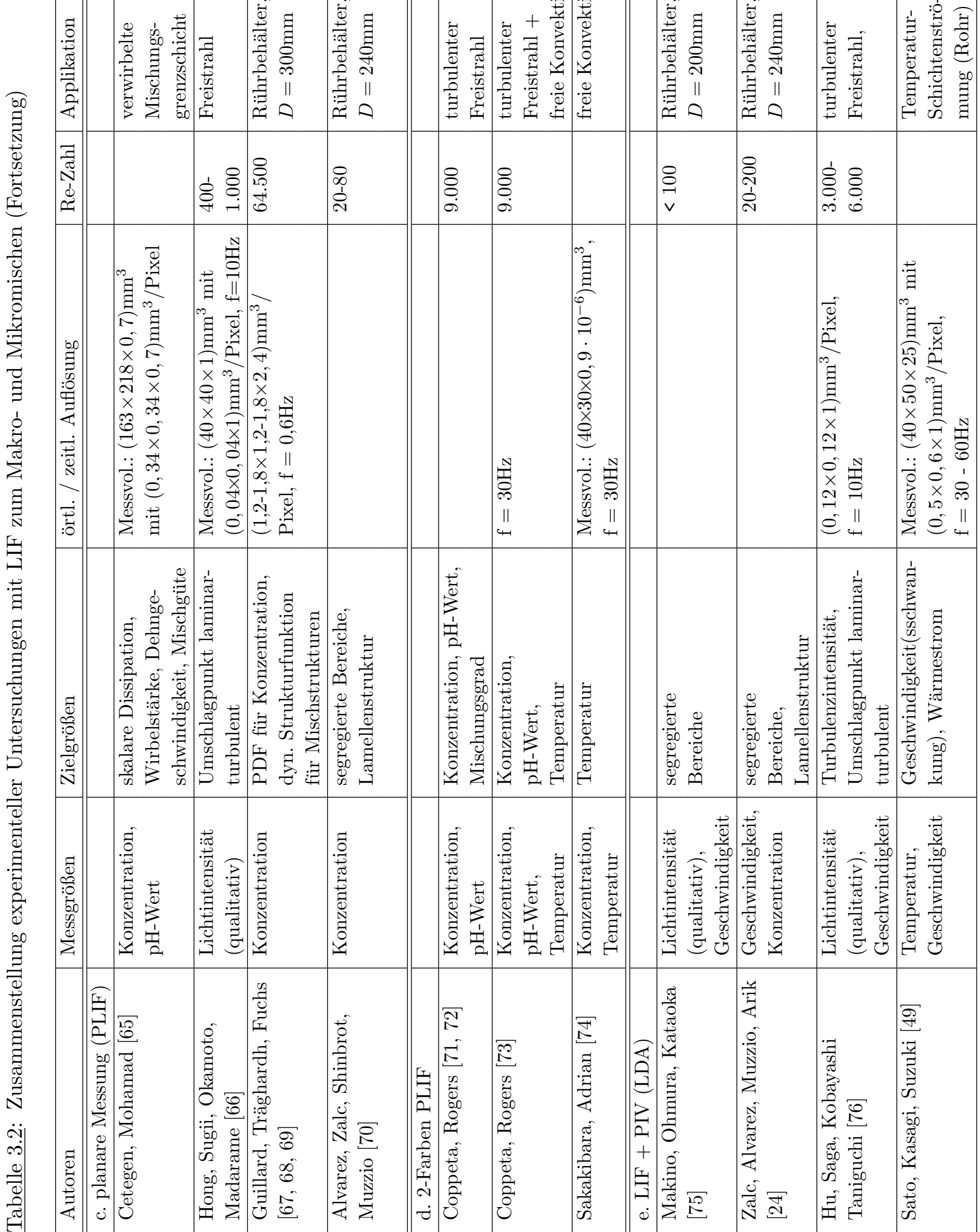


des Laserstrahls, also für eine horizontale oder vertikale Linie gemessen werden. Die Messungen werden gestartet, wenn sich ein Rührerblatt parallel zur Messlinie befindet und alle $5^{\circ}$ wiederholt, so dass winkel-aufgelöste Messungen möglich sind. Die Aufnahmefrequenz liegt dabei im Kilohertz-Bereich. Periodische Konzentrationsschwankungen bedingt durch die Wirbelschleppen hinter den Rührerblättern können so sichtbar gemacht werden. Als Maß für die Makromischung berechnen die Autoren die Abweichung der lokalen Konzentration von einer mittleren Konzentration, während eine normalisierte Standardabweichung der Konzentrationsdifferenz als Maß für die Mikromischung dient. Guillard, Trägardh und Fuchs [67, 68] verwenden die PLIF zum Visualisieren der kleinskaligen Wirbelstrukturen im turbulenten Strömungsfeld um einen Scheibenrührer. Mit Hilfe der Methoden der automatischen Bildverarbeitung können die Strukturen detektiert und der zeitliche Verlauf beobachtet werden. Lokale Oszillationen der Wirbelstrukturen, die sowohl auf kleinen als auch auf großen Zeitskalen (z.B. 1s bzw. 80s) existieren, werden mit Hilfe von Wahrscheinlichkeitsdichtefunktionen beschrieben.

Der Ablauf der laminaren Vermischung in einem Rührbehälter mit drei Scheibenrührern wird von Muzzio und Mitarbeitern untersucht [70]. Das Konzentrationsfeld eines inerten Tracers wird mit Hilfe der PLIF gemessen. Dabei sind lamellenartige Strukturen zu beobachten. Das Geschwindigkeitsfeld wird mit Hilfe der PIV gemessen. Die experimentellen Ergebnisse dienen zur Validierung eines CFD-Programms, das auf der Methode der Finiten Elemente beruht und speziell für die Berechnung von Mischprozessen optimiert ist [24, 22]. Die Kombination der beiden Messmethoden Planare Laserinduzierte Fluoreszenz (PLIF) und Particle Image Velocimetry (PIV) erlaubt es, die Konzentrations- und die Geschwindigkeitsfelder in der gleichen Messebene zu messen (Hu et al. [76]). Sato, Kasagi und Suzuki [49] verwenden beide Messtechniken gleichzeitig, um das Temperaturfeld und die Geschwindigkeiten in einer turbulenten Mischungsgrenzschicht zu bestimmen. Law und Wang [79] untersuchen, wie die Messergebnisse durch die gleichzeitige Verwendung der Messmethoden beeinflusst werden.

Chemisch reagierende Tracer werden verwendet, um das Mischen im Bereich molekularer Längenskalen auf indirektem Wege sichtbar zu machen. Dabei werden fast ausschließlich pH-Wert abhängige Farbstoffe eingesetzt. Einige Forschergruppen nutzen dabei aus, dass der Fluoreszenzfarbstoff Fluorescein nur in basischer oder schwach saurer Umgebung mit $\mathrm{pH}>4$ Fluoreszenzlicht emittiert. Makino, Ohmura und Kataoka [75] verwenden den Farbstoff zur rein qualitativen Visualisierung der Vermischung in Rührbehältern. Dabei wird Fluorescein in basischer Umgebung im Behälter vorgelegt und durch Zugabe von Säure entfärbt. So bleiben nur isolierte Mischzonen sichtbar. Die Autoren finden im Querschnitt der torusförmigen isolierten Mischzone eine komplexe Struktur aus mehreren Fäden, die um ein Zentrum angeordnet sind. Ihre Zahl ist von der Drehzahl und der 
Anzahl der Rührerblätter abhängig. Cetegen und Mohamad [65] messen dagegen quantitativ die Produktkonzentration, die unter bestimmten Voraussetzungen proportional zur gemessenen Fluoreszenzintensität ist. Bellerose und Rogers [64] messen die pH-WertVerteilung in einem turbulenten Freistrahl. Hong und Mitarbeiter [66] untersuchen den Einfluss der Säure-Base-Reaktion auf die Ausbreitung des Strahls verglichen zur Strahlströmung rein inerter Komponenten. Ein prinzipieller Nachteil bei der Verwendung eines einzelnen reagierenden Tracers ist, dass Makro- und Mikrovermischung nicht voneinander getrennt werden können. Regionen ohne Reaktionsprodukt sind entweder auf mikroskopischen Skalen noch unvermischt oder aber auch makroskopisch segregiert. Diese Methode ist deshalb streng genommen nur bei idealer Makrovermischung aussagekräftig, was bei laminarer Strömung nicht der Fall ist.

André und Mitarbeiter [53] verwenden ein Gemisch aus zwei inerten Tracern, wobei einer ein fluoreszierender Farbstoff ist, während der andere dessen Fluoreszenz hemmt. Die Vermischung auf molekularer Ebene wird so sichtbar gemacht, ohne dass eine chemische Umsetzung der Komponenten erfolgt. Die Konzentrationsschwankungen der beiden Tracer wird an diskreten Orten im Rührbehälter gemessen und daraus ein Segregationsindex berechnet. Dieser wird mit Ergebnissen bei der Verwendung eines einzelnen, inerten Tracers (s.o., [55]) verglichen [54].

Coppeta und Rogers [73, 72] und Sakakibara und Adrian [74] verwenden zwei Fluoreszenzfarbstoffe, um Temperaturfelder oder eine Verteilung des pH-Werts zu messen. Dazu wird ein Farbstoff ausgewählt, dessen Emissionscharakteristik von einem dieser Parameter abhängt, während der andere Farbstoff inert ist. Das Signal des inerten Farbstoffs dient als Norm, und nur das Verhältnis der bei verschiedenen Wellenlängen gemessenen Fluoreszenzintensitäten wird in Abhängigkeit von pH-Wert oder Temperatur kalibriert. Dieses Verhältnis ist unabhängig von Konzentrationsänderungen, Schwankungen der Laserleistung und der Absorption entlang des Strahlwegs. Auf diese Weise sind aufwändige Korrekturen der Messwerte unnötig. In [73] sind die spektralen Eigenschaften von neun wasserlöslichen Fluoreszenzfarbstoffen und Kriterien für die Auswahl eines Farbstoffgemisches angegeben. Einige Fluoreszenzfarbstoffe fluoreszieren auf zwei unterschiedlichen ausgeprägten Wellenlängen. Ändert sich das Verhältnis der auf diesen Wellenlängen gemessenen Intensitäten mit der Temperatur oder dem pH-Wert, so ist für die oben beschriebene Verhältnismessung nur ein Farbstoff notwendig [71].

Die lokale Mikromischgüte wurde unter Berücksichtigung des Makrotransports experimentell bisher nur von Buchmann untersucht, wobei die örtliche Diskretisierung der Messtechnik mindestens eine Größenordnung über dem Längenmaßstab der Segregation liegt. Die Trennung von Makro- und Mikrovermischung ist während der Experimente von Coppeta und Rogers $[73,72]$ trotz der Verwendung von zwei Farbstoffen nicht möglich, da 
bei dieser Methode über den pH-Wert nur die Konzentration eines einzelnen Tracers, der Hydroniumionen, gemessen wird. Somit bleiben die oben erwähnten Nachteile bei der Verwendung eines einzelnen reagierenden Tracers bestehen. Um den Zusammenhang zwischen Strömungsfeld, örtlicher Mischgüte und chemischer Reaktion zu untersuchen, sind weitere Experimente erforderlich.

\subsection{Strömungsfelder und Energiedissipation in gerührten Behältern}

Die Güte der Mikrovermischung hängt vom Scherfeld und damit vom lokalen Geschwindigkeitsgradienten im Mischbehälter ab. Das Geschwindigkeitsfeld ist vom makroskopischen Energieeintrag durch den Rührer abhängig und lokal sehr unterschiedlich. Die im Behälter dissipierte Leistung wird häufig zum Dimensionieren der Antriebsleistung des Rührers verwendet und ist deshalb für viele unterschiedliche Rührer und Flüssigkeiten aus experimentellen Untersuchungen bekannt (z.B. [80] [81], [82], [83], [84], [85], [86]). Für viele Anwendungen sind volumetrisch und zeitlich gemittelte Aussagen zum Leistungseintrag aber nicht ausreichend. Beispielsweise dürfen in Bioreaktoren bestimmte Scherraten lokal nicht überschritten werden. Auch bei gleichzeitigem Wärme- und Stoffaustausch ist es notwendig, die lokalen Geschwindigkeiten und die daraus resultierenden lokal dissipierten Energieströme zu kennen.

\subsubsection{Numerische Ergebnisse}

Die numerische Berechnung laminarer und turbulenter Strömungsfelder ist in den letzten Jahren besonders aufgrund der zunehmenden Rechnerleistungen stark verbessert worden. Numerische Strömungsberechnungen haben den Vorteil, dass lokale Informationen über Konzentrationen, Temperatur, Strömungsgeschwindigkeiten, Turbulenz, Wärmeund Stoffaustausch und eventuell ablaufende chemische Reaktionen erhalten werden können. Der Einfluss von Geometrie- und Betriebsparametern kann mittels Computational Fluid Dynamics (CFD) unabhängig voneinander untersucht werden, was durch experimentelle Untersuchungen häufig schwierig ist. Allerdings werden dabei meist nur qualitative Ergebnisse erzielt. Die quantitative Vorhersagbarkeit von Strömungsprozessen mittels CFD ist besonders durch die Verwendung von vereinfachenden Modellen z.B. für die Turbulenz begrenzt. Bakker, Haidari und Oshinowo [87] sowie Souvaliotis, Jana und Ottino [88] schätzen die Möglichkeiten der CFD ab und zeigen Fehlerquellen auf. Birtigh und Mitarbeiter [89] geben aus industrieller Sicht einen Überblick über das Potenzial der CFD als Design-Tool. 
Wie bereits in Kapitel 3.1.1 erläutert, können die Transportvorgänge im Mikromaßstab der Ortskoordinaten prinzipiell mit Hilfe der Direkten Numerischen Simulation (DNS) berechnet werden. Die Rechenzeiten nehmen aber nur für einfach berandete Strömungsfelder und relativ kleine Re-Zahlen vertretbare Werte an. Nicht so aufwendig wie die DNS und somit für etwas komplexere Geometrien geeignet, ist das Verfahren der Grobstrukturoder Large-Eddy-Simulation (LES). Dabei werden die Transportvorgänge auf den Skalen, die kleiner als das Rechengitter sind, herausgefiltert und ihr Effekt auf die größeren Skalen modelliert, während letztere direkt berechnet werden. Zhao, Brodkey und Nakamura [90] haben so die Vermischung durch zwei aufeinander prallende Freistrahlen berechnet. Für Geometrien mit bewegten Grenzfächen, wie z.B. in einem Rührbehälter mit dynamischem Rührorgan, ist die LES- Simulation noch nicht Stand der Technik. Revstedt und Fuchs [91] berechnen das Geschwindigkeitsfeld in einem Rührbehälter mit zwei unterschiedlichen Rührorganen und vergleichen ihre Ergebnisse mit experimentellen Daten. Dabei werden zufrieden stellende Übereinstimmungen erzielt. Am häufigsten kommen aber noch immer Verfahren zum Einsatz, die auf der Lösung der Reynoldsgemittelten Navier-Stokes-Gleichungen (RANS) in Verbindung mit einem geeigneten Turbulenzmodell basieren. Am weitesten verbreitet ist dabei das $k$ - $\epsilon$-Modell, das den Transport der turbulenten kinetischen Energie $k$ und der Dissipationsrate $\epsilon$ berücksichtigt. Die zwei Diffenrentialgleichungen enthalten fünf Konstanten, die empirisch ermittelt werden. Sahu und Mitarbeiter [92] verwenden unterschiedliche Gruppen von Konstanten in Abhängigkeit vom Ort im Behälter und können so verbesserte Ergebnisse erzielen.

Das $k$ - $\epsilon$-Modell gilt streng genommen nur für isotrope Turbulenz. Die Anisotropie der Turbulenz kann mit Reynoldsspannungsmodellen erfasst werden. Diese Klasse der Turbulenzmodelle ist allerdings sehr komplex und erfordert das Lösen von sechs Differentialgleichungen für den Transport der turbulenten Spannungen [25]. Sheng, Meng und Fox [93] verwenden das $k$ - $\epsilon$-Modell und das Reynoldsspannungsmodell und stellen fest, dass für die mittlere Geschwindigkeitsverteilung von beiden Modellen ähnlich gute Ergebnisse erzielt werden. Reynoldspannungen sind nur mit dem Reynoldsspannungsmodell vorhersagbar, wobei aber üblicherweise zu kleine Werte berechnet werden. Grundsätzlich werden die Werte für $k$ und $\epsilon$ besonders in der Strömung weiter entfernt von der Rührerebene unterschätzt. Die Autoren vergleichen ihre Ergebnisse mit experimentellen Daten, die mit Hilfe der Particle-Image-Velocimetry (PIV) und der Laser-Doppler-Anemometrie (LDA) gewonnen werden.

Bei der Betrachtung laminarer Strömungen ist die Modellierung der turbulenten kinetischen Energie nicht notwendig, so dass sie zunächst leichter berechenbar scheinen. Allerdings ist die sogenannte numerische Diffusion bei laminaren Strömungen meist sehr ausgeprägt, so dass auf eine ausreichende örtliche und zeitliche Diskretisierung geachtet werden 
muss [88]. Für die Berechnung der Strömung nicht-newtonscher Fluide muss außerdem die Abhängigkeit der Viskosität von der Scherrate über einen geeigneten Ansatz modelliert werden $[94,95]$.

\subsubsection{Experimentelle Ergebnisse}

Die Überprüfung und Kalibrierung der numerischen Ergebnisse mit örtlich und zeitlich hoch aufgelösten Messergebnissen ist weiterhin unerlässlich. Bryanston-Cross und Mitarbeiter [96] geben einen guten Überblick über derzeit zur Verfügung stehende Messverfahren zum Messen von Strömungsfeldern. Aufgrund der hohen zeitlichen und örtlichen Auflösung war die Laser-Doppler-Anemometrie (LDA) lange Zeit das am häufigsten verwendete Verfahren zum Messen von lokalen Geschwindigkeiten und Geschwindigkeitsschwankungen [97]. Das hierbei beobachtete Messvolumen ist quasi punktförmig, so dass der Messraum in mehreren zeitlich konsekutiven Messungen abgetastet werden muss. Momentane örtliche Geschwindigkeitsgradienten und die davon abhängige Dissipation der kinetischen Energie sind deshalb nicht direkt messbar. Brodkey [98] gibt einen halbempirischen Ansatz zur Berechnung der lokalen Energiedissipationsrate $\epsilon$ in Abhängigkeit von der lokalen Schwankungsgeschwindigkeit im turbulent gerührten Fluid an. Laufhütte und Mersmann [99] messen mit Hilfe der LDA die Felder der turbulenten Schwankungsgeschwindigkeit für unterschiedliche Rührer und ermitteln daraus die Felder der lokalen Energiedissipationsraten im Gefäß nach der Methode von Brodkey. Rührerorgane mit einem kleinen Verhältnis von Rührer- zu Behälterdurchmesser dissipieren einen großen Teil der Energie in ihrer Nähe. Größere Rührerdurchmesser führen dazu, dass die Energie gleichmäßiger im ganzen Rührgefäß dissipiert wird. Werner und Mersmann [100] nehmen eine fraktale Struktur der Turbulenz an und messen die Schwankungsgrößen der Geschwindigkeit für newtonsche und strukturviskose Flüssigkeiten. Daraus berechnen die Autoren die Brodkey-Konstante, die die Energiedissipationsrate mit der Schwankungsgeschwindigkeit und einem makroskopischen Längenmaßstab verbindet. Die gemessenen und berechneten Werte stimmen sowohl für newtonsche als auch für nicht-newtonsche Flüssigkeiten überein.

Die großräumige Strömung und der Vergleich mit numerischen Berechnungen stehen im Mittelpunkt der Untersuchungen von Sheng, Meng und Fox [93] und von Montante, Lee, Brucato und Yianneskis [101]. Moore, Cossor und Baker [102] setzen die LDA-Technik ein, um die lokalen Geschwindigkeiten in einer durchmischten Carboxy-Vinyl-Polymerlösung zu messen. Sie ermitteln daraus die Größe der Kaverne, die sich für strukturviskose Fluide in der Umgebung des Rührorgans bildet. Schäfer, Yianneskis, Wächter und Durst [103] messen das Geschwindigkeitsfeld direkt hinter den Rührerblättern mittels LDA. Die Mes- 
sungen werden mit der Drehbewegung des Rührers getriggert, so dass die Messdaten einer Position relativ zu den Rührerblättern zugeordnet werden kann. Die Wirbelschleppe im Nachlauf eines Schrägblattrührers kann so, örtlich sehr gut aufgelöst, visualisiert werden. Aufgrund der verstärkten Forschungstätigkeit in den letzten Jahren ist es möglich, diese örtliche Auflösung auch mit der Particle Image Velocimetry (PIV) zu erreichen. Dabei handelt es sich wie bei der Planaren Laserinduzierten Fluoreszenz (PLIF) um ein Lichtschnittverfahren, so dass lokale Geschwindigkeiten zeitgleich an vielen Punkten in der Messebene gemessen werden können. Ist die örtliche Auflösung groß genug um die Kolmogorov-Skala aufzulösen, können die lokalen Geschwindigkeitsgradienten direkt aus den momentanen Geschwindigkeiten berechnet werden. Sharp und Adrian [104] sowie Ranade, Perrard, Sauze, Xuereb und Bertrand [105] verwenden die Particle Image Velocimetry (PIV) zum Messen des Geschwindigkeitsfelds in der unmittelbaren Umgebung eines Scheibenrührers. Dabei werden nur die beiden Geschwindigkeitskomponenten in der vertikalen bzw. horizontalen Messebene erfasst. Die örtliche Auflösung ist so gewählt, dass Wirbelstrukturen, die durch Ablösung an den Rührerblättern entstehen, örtlich aufgelöst werden können. Unter Annahme isotroper Turbulenz berechnen Sharp und Adrian das Feld der lokalen Energiedissipation direkt aus den örtlichen Gradienten der in der Ebene gemessenen Schwankungsgeschwindigkeiten. Für eine direkte Berechnung der lokalen Energiedissipation ist die örtliche Auflösung der von Escudié und Liné [106] mit PIV gemessenen Geschwindigkeiten nicht ausreichend, so dass sie die lokale Energiedissipation aus der Bilanzgleichung für die turbulente kinetische Energie abschätzen. Baldi und Yianneskis [107] messen zusätzlich die dritte Geschwindigkeitskomponente senkrecht zur Bildebene mit Hilfe der sogenannten Stereo-PIV. Dabei wird ein verhältnismäßig dicker Lichtschnitt von etwa $1 \mathrm{~mm}$ verwendet und die Bildebene von zwei nebeneinander angeordneten Kameras beobachtet. Dadurch entsteht, ähnlich wie beim menschlichen Sehen, ein räumlicher Eindruck. Bedingt durch den dicken Lichtschnitt ist die örtliche Auflösung in der dritten Richtung aber so gering, dass der örtliche Geschwindigkeitsgradient nicht direkt aus den Messwerten berechnet werden kann. Die Autoren wenden zum Berechnen der lokalen Energiedissipation deshalb das gleiche Verfahren wie Sharp und Adrian an.

Mit dem Verfahren der holographischen PIV ist es möglich, die drei Komponenten des Geschwindigkeitsvektors zeitgleich in einem größeren Volumen zu messen. Auf diese Weise könnte die lokale Energiedissipation direkt gemessen werden. Eine detaillierte Beschreibung der Grundlagen der Messmethode und einen Überblick der hierzu publizierten Ergebnisse aus bekannten Forschungsarbeiten gibt Hinsch [108]. Allerdings ist das relativ junge Messverfahren ist noch nicht ausgereift entwickelt, so dass Messungen in technisch interessanten Strömungen sehr aufwändig sind.

Die zeitliche Auflösung der PIV ist klassischerweise wesentlich geringer als bei der LDA 
und liegt in der Größenordnung von $10 \mathrm{~Hz}$. Die Entwicklung digitaler Kameras und gepulster Laser mit Wiederholraten im kHz-Bereich lässt inzwischen aber auch zeitlich aufgelöste Geschwindigkeitsmessungen zu [109]. 


\section{Visualisieren des Mikro- und Makromischens mit Hilfe von Tracern}

Werden zwei Flüssigkeiten im laminaren Strömungsfeld gemischt, so tritt die Homogenisierung im molekularen Maßstab erst ein, wenn quer zu den Stromlinien eine Vermischung durch Diffusion erfolgt. Enthalten die Flüssigkeiten gleichzeitig inerte und chemisch reagierende Tracer, so ist der in Bild 4.1 schematisch für eine Entfärbungsreaktion dargestellte Ablauf der Vermischung zu beobachten. Zu Beginn des Mischprozesses werden beide Farbstoffe durch Konvektion im Behälter transportiert. Die eingebrachten Fluidelemente werden dabei im Strömungsfeld gestreckt, gefaltet und gedreht, wobei der Längenmaßstab der Segregation, d.h. die Lamellendicke, reduziert wird. Dabei werden zunächst Lamellendicken erreicht, für die diffusive Transportvorgänge an der Lamellenoberfläche eine nur untergeordnete Rolle spielen. Eine Vermischung der Fluidelemente mit der umgebenden Flüssigkeit findet im molekularen Skalenbereich der Ortskoordinate noch nicht statt. Eine chemische Reaktion kann noch nicht ablaufen, weil hierfür die molekulare Vermischung erforderlich ist. Im weiteren Verlauf des Mischens nimmt die Längenskala der Segregation jedoch weiter ab, so dass große Konzentrationsgradienten den Ausgleich der molekularen Konzentrationsunterschiede ermöglichen, wobei die Intensität der Segregation abnimmt. Im Skalenbereich der molekularen Abmessungen findet dann die chemische Reaktion statt, so dass der beobachtete Umsatz ein indirektes Maß für die Mikrovermischung darstellt. Nach abgeschlossener Vermischung sind der inerte Farbstoff und das Reaktionsprodukt im molekularen Skalenbereich der Ortskoordinate homogen verteilt. Findet eine Entfärbungsreaktion statt, so ist nur noch der inerte Farbstoff sichtbar. Das lokale Konzentrationsverhältnis aus Reaktionsprodukt und inertem Farbstoff kann somit als Maß für die Intensität der Segregation dienen. Analog zu dem von Danckwerts [5] vorgeschlagenen Begriff der Intensität der Segregation $I_{S}$ (Gl. (3.1) definiert Käppel [27] den globalen Deviations$\operatorname{grad}$

$$
\Delta_{g l o b}(t)=\frac{\alpha(t)}{\alpha_{0}}=\frac{\sum_{i=1}^{N}\left|c^{i}(t)-\bar{c}\right|}{\sum_{i=1}^{N}\left|c_{0}^{i}-\bar{c}\right|} .
$$

wobei statt der Varianz $\sigma^{2}$ (zweites zentrales Moment der Konzentrationsverteilung) das erste absolute zentrale Moment der Konzentrationsverteilung $\alpha$ verwendet wird. $N$ ist die 


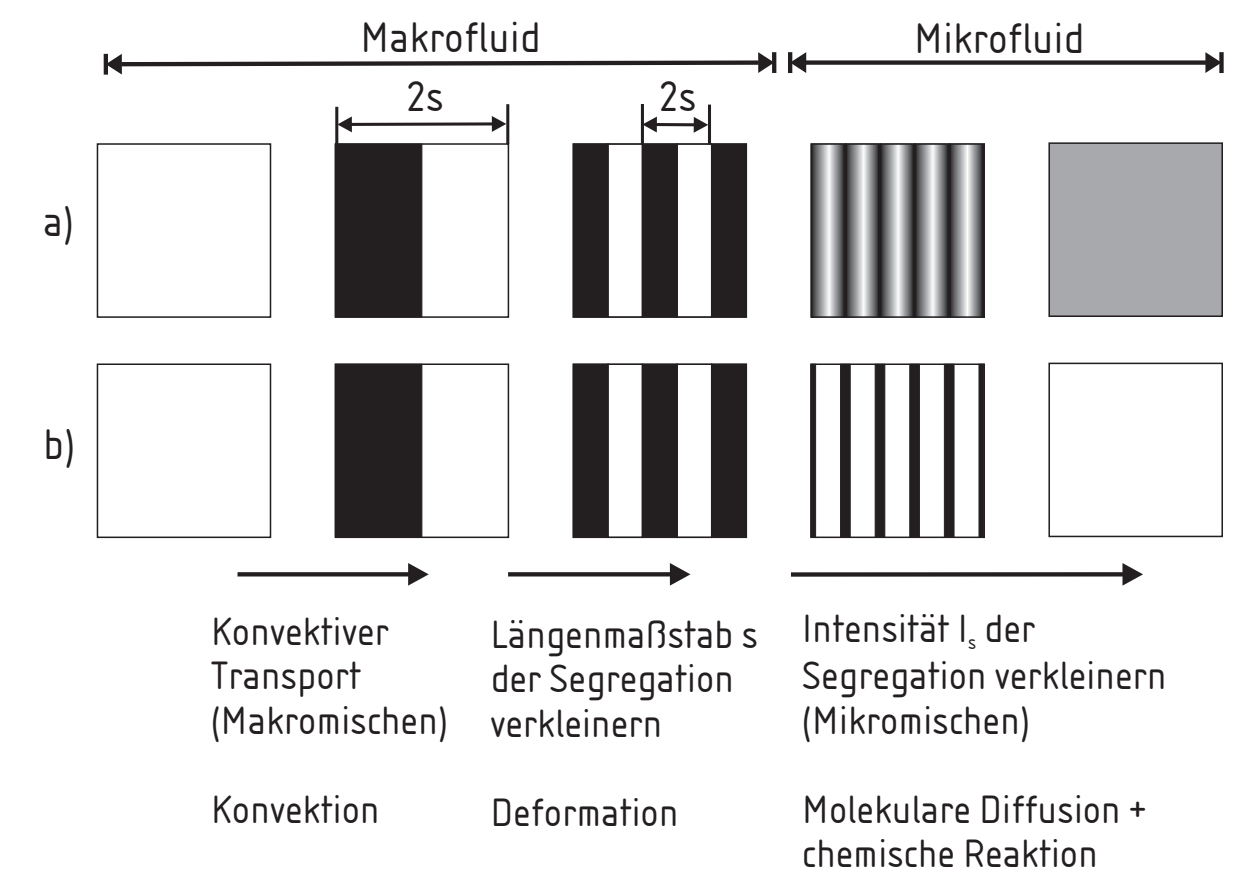

Bild 4.1: Schematischer Ablauf des laminaren Mischvorgangs für a) inerte und b) sich entfärbende Komponente

Anzahl der Probenvolumen $V_{P}$. Käppel hat gezeigt, dass der Deviationsgrad

$$
\Delta_{g l o b}(t)=\frac{c_{1}(t)}{c_{1,0}}
$$

gleich dem Verhältnis aus der Konzentration $c_{1}$ eines im Rührgefäß homogen verteilten reagierenden und sich entfärbenden Farbstoffs $1 \mathrm{zu}$ einer beliebigen Zeit $t$ und dessen Konzentration $c_{1,0}$ zum Zeitpunkt der Zugabe des Reaktanden ist, der stöchiometrisch zugegeben wird. Wird statt der Entfärbungsreaktion eine beliebige, schnell ablaufende chemische Reaktion gewählt, für die das Reaktionsprodukt $R$ anstelle des Reaktanden nachgewiesen werden kann, so kann die Anfangskonzentration

$$
c_{1,0}=\nu_{1} c_{1}(t)+\nu_{1, R} c_{1, R}(t)
$$

als Summe der zeitlich veränderlichen Konzentrationen des Reaktanden $c_{1}(t)$ und des Reaktionsprodukts $c_{1, R}(t)$ berechnet werden. Dabei sind $\nu_{1}$ und $\nu_{1, R}$ die stöchiometrischen Zahlen des Reaktanden und des Reaktionsprodukts in der Reaktion. Im Folgenden werden diese ohne Beschränkung der Allgemeinheit vereinfachend zu Eins gesetzt. Der Deviationsgrad ergibt sich damit gemäß Gl. (4.2)

$$
\Delta_{\text {glob }}(t)=\frac{c_{1}(t)}{c_{1,0}}=\frac{c_{1,0}-c_{1, R}(t)}{c_{1,0}}=1-\frac{c_{1, R}(t)}{c_{1,0}} .
$$

Diese Methode erlaubt allerdings nur integrale Messungen, da die anfängliche Konzentration eines eingespritzten Tropfens an einer bestimmten Stelle des Rührgefäßes gemäß der vorigen Definition unbekannt ist. Die lokale Konzentration des Ausgangsstoffes $c_{1}(t)$ wird 
von zwei Mechanismen beeinflusst. Zum einen wird die Konzentration im eingespritzten Tropfen durch den konvektiven Transport auch ohne Diffusion, d.h. Mikrovermischung verdünnt. Nach der Mikrovermischung führt die chemische Reaktion zusätzlich zu einer Abnahme der Konzentration $c_{1}$. Diese gleichzeitig ablaufenden Mechanismen können durch Zugabe eines Gemisches aus einem inerten Farbstoff 2 und einem chemisch reagierenden Farbstoff 1 optisch voneinander getrennt werden. Der Verdünnungseffekt betrifft beide Farbstoffe, während die Mikrovermischung nur die Konzentration des reagierenden Farbstoffs beeinflusst. Analog zu Gl. (4.4) wird der lokale Deviationsgrad

$$
\Delta(\vec{x}, t)=1-\frac{c_{1, R}(\vec{x}, t)}{c_{1}(\vec{x}, t)}
$$

definiert. Dabei kann die lokale Konzentration des reagierenden Farbstoffs

$$
c_{1}(\vec{x}, t)=c_{2}(\vec{x}, t) \frac{c_{1,0}}{c_{2,0}}
$$

die dieser ohne Abbau durch die chemische Reaktion an dem entsprechenden Ort hätte, aus der lokalen Konzentration des inerten Farbstoffs $c_{2}(\vec{x}, t)$ und dem Anfangskonzentrationsverhältnis $C_{0}=\frac{c_{1,0}}{c_{2,0}}$ berechnet werden.

Der lokale Deviationsgrad ist gleich dem Anteil des reagierenden Farbstoffs, der noch nicht umgesetzt ist und besitzt einen ähnlichen Zeitverlauf wie die Intensität der Segregation. Er fällt von Eins, was vollständiger Segregation auf makroskopischer Ebene entspricht, auf den Wert Null für ein vollständig gleichmäßig mikrovermischtes Fluid. Unter bestimmten Annahmen ist es möglich, die beiden Maße ineinander umzurechnen. Hiby [110] zeigt die Zusammenhänge zwischen verschiedenen Mischgütemaßen, wie z.B. der Standardabweichung, dem Deviationsgrad und dem Segregationsgrad. Um aber die lokalen Messergebnisse unverfälscht wiederzugeben, wird in dieser Arbeit nur der lokale Deviationsgrad wie oben definiert verwendet.

Bei der Auswahl eines geeigneten Mischgütemaßes ist grundsätzlich zu beachten, dass dieses für ein bestimmtes Probenvolumen definiert ist und abhängig von dessen volumetrischer Ausdehnung unterschiedliche Werte liefert [27]. Um dies zu veranschaulichen, genügt es die beiden Extremfälle zu betrachten:

1. Probenvolumen $V_{P}=$ Gesamtvolumen $V_{\text {ges }}$

2. Probenvolumen $V_{P}=$ Molekülvolumen $V_{m o l}$

Für eine Mischung aus den zwei Komponenten A und B ist der Volumenanteil der Komponente $\mathrm{A}$ in einer Probe

$$
c_{A, P}=\frac{V_{A, P}}{V_{P}}
$$


für Komponente B gilt Gl. (4.7) analog. Der Mittelwert von A (oder analog von B) ist:

$$
\bar{c}_{A}=\frac{V_{A}}{V_{\text {ges }}} .
$$

Damit ist für den ersten Extremfall mit $V_{A, P}=V_{A}$ und $V_{P}=V_{\text {ges }}$ der Volumenanteil von $\mathrm{A}$ zu jeder Zeit der Mischung gleich dem Mittelwert: $c_{A, P}=\bar{c}_{A}$. Die Abweichung zwischen $c_{A, P}$ und $\bar{c}_{A}$ ist damit minimal und gleich Null. Für den zweiten Extremfall, bei dem jedes Molekül eine Probe darstellt, gilt hingegen für jedes Probenvolumen immer entweder $c_{A, P}=0$ oder $c_{A, P}=1$. Die Abweichung zum Mittelwert ist damit maximal. Für denselben Mischungszustand nimmt diese Abweichung je nach Probengröße Werte zwischen diesen Extremwerten an. Dieses einfache Beispiel verdeutlicht, dass beim Vergleich zwischen experimentellen Ergebnissen bzw. zwischen Experiment und Simulation darauf geachtet werden muss, dass das Mischgütemaß (hier: der lokale Deviationsgrad) für gleiche Probenvolumen berechnet wird. Eine Probengröße, die zu einem aussagekräftigen Mischgütemaß führt, ist deutlich kleiner als der makroskopische Längenmaßstab der Segregation (im laminaren Fall die Lamellendicke) aber größer als der molekulare Maßstab. Für experimentelle Untersuchungen ist die Probengröße technisch begrenzt. In dieser Arbeit wird eine örtliche Aufösung gewählt, für die zumindest in der Anfangszeit der Vermischung das Probenvolumen kleiner als der Längenmaßstab der Segregation ist (siehe Kapitel 9). 


\section{Fluoreszenzspektroskopische Messungen}

Emittiert eine Substanz elektromagnetische Strahlung als Folge der Absorption ultravioletten, sichtbaren oder infraroten Lichts, so spricht man von Photolumineszenz. Je nach Dauer des Abstrahlvorganges unterscheidet man bei der Photolumineszenz zwischen Fluoreszenz und Phosphoreszenz. Während die Fluoreszenzemission innerhalb von $10^{-9} \mathrm{~s}$ bis $10^{-6} \mathrm{~s}$ abklingt, überdauert die Lichtemission der Phosphoreszenz die Anregung um $10^{-4}$ s bis 100 s oder noch länger. Im Folgenden wird kurz die Entstehung der Fluoreszenzemission erläutert. Danach werden in Kapitel 5.1 und 5.2 spektroskopische Messungen sowohl mit dem Fluoreszenz- als auch mit dem Absorptionspektrometer erläutert, bevor in Kapitel 5.3 auf die quantitative Analyse der Messwerte eingegangen wird.

Durch Absorption von Licht ändert sich die Energie der Elektronen auf den äußeren Schalen der Atome bzw. der Moleküle. Diese Energien sind Quantenzuständen zugeordnet und lassen sich durch ein sogenanntes Termschema einzelnen Niveaus zuordnen, welche stark vereinfacht in Bild 5.1 abgebildet sind.

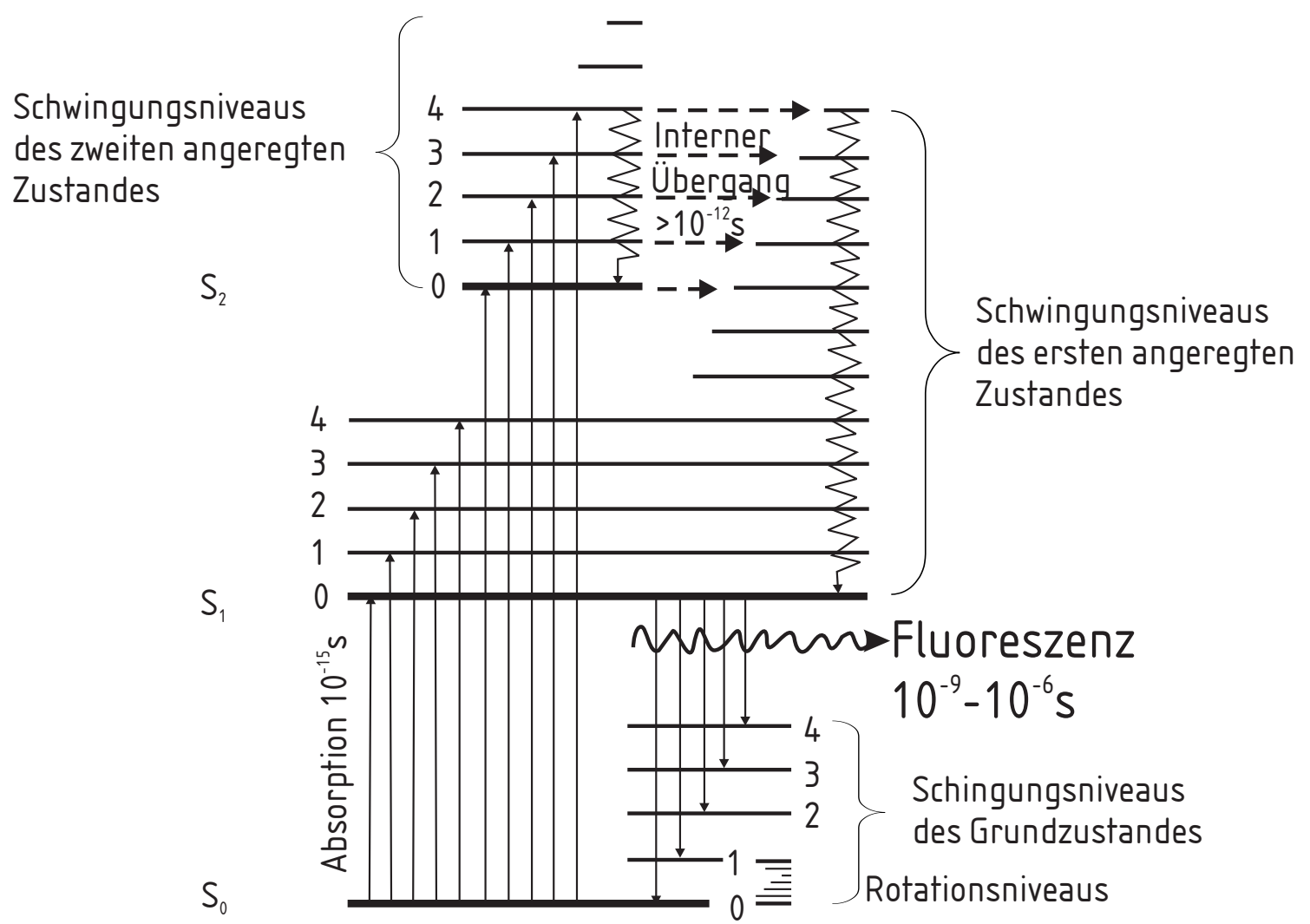

Bild 5.1: Schematische Darstellung der Energiezustände eines Atoms bzw. Moleküls 
Bei Raumtemperatur befinden sich die meisten Moleküle im Grundzustand $S_{0}$. Durch Energieabsorption können die Moleküle entweder den ersten $\left(S_{1}\right)$ oder den zweiten $\left(S_{2}\right)$ angeregten Zustand erreichen. Durch Absorption von Licht kann ein Molekül jedes der Energieniveaus, die als Unterniveaus der einzelnen angeregten Schwingungszustände aufzufassen sind, erreichen. Da die Energie aber in Quanten absorbiert wird, sollte bei einem reinen Stoff eine Reihe deutlich getrennter Absorptionsbanden zu beobachten sein. Das obige Schema vernachlässigt allerdings die Tatsache, dass die Energieniveaus noch in feinere, der Rotation zugeordnete Niveaus unterteilt sind. Dadurch erhöht sich die Anzahl möglicher Absorptionsbanden so stark, dass eine Auflösung der Feinstruktur im Spektrum der meisten Substanzen nicht möglich ist. Durch diesen Effekt sowie durch Lösungsmitteleinflüsse beobachtet man meist breitbandige Absorptionsspektren.

Nachdem das Molekül Energie absorbiert hat und damit eines der oberen Energieniveaus eines angeregten Zustands erreicht hat, verliert es sehr schnell durch Molekülstöße den Überschuss an Energie und fällt auf das niederste Niveau des angeregten Zustands zurück. Zusätzlich können interne Übergänge auf energiegleiche höhere Niveaus eines niedrigeren Anregungszustands erfolgen. Von dort verlieren die Moleküle wieder Energie durch Molekülstöße bis sie das unterste Niveau des ersten angeregten Zustands erreicht haben.

Vom ersten angeregten Zustand $S_{1}$ kann das Molekül auf eines der Energieniveaus des Grundzustands $S_{0}$ zurückfallen. Dabei verliert es Energie, die jetzt in Form von elektromagnetischer Strahlung, dem Fluoreszenzlicht, abgegeben werden kann. Im allgemeinen gibt nur ein Teil der Moleküle die absorbierte Energie in Form von Fluoreszenz ab, die restliche Energie wird strahlungslos in Wärme dissipiert. Diese strahlungslose Energieabgabe aufgrund von Wechselwirkungen mit anderen Molekülen in der Umgebung wird Quenching (aus dem englischen: to quench = löschen, d.h. Fluoreszenzlöschung) genannt. Welcher Bruchteil der angeregten Moleküle ihre Energie als Fluoreszenzemission abgeben, hängt also von der Art der Umgebung ab. Dazu gehört z.B. die Zusammensetzung des Lösungsmittels, die Konzentration des Fluorophors und die Temperatur. Das Verhältnis aus der Lichtintensität des emittierten Lichts und der Lichtintensität des absorbierten Lichts wird als Quanteneffizienz $\Phi$ bezeichnet. Sie kann Werte zwischen Null und Eins annehmen. Für den Grenzfall $\Phi=1$ wird die Energie vollständig in Form von Fluoreszenzstrahlung abgegeben und der Prozess verläuft reversibel. Für den zweiten Grenzfall $\Phi=0$ erfolgt die Energieabgabe hingegen vollständig strahlungsfrei.

Der Übergang vom untersten Energieniveau des Grundzustands zum untersten Energieniveau des ersten angeregten Zustands ist energetisch für Absorptions- und Emissionsphänomene gleich. Alle anderen Übergänge benötigen mehr Energie bei der Absorption als jeglicher Übergang bei der Fluoreszenzemission. Das Emissionsspektrum ist gegenüber dem Absorptionsspektrum entsprechend zu der Seite der geringeren Energie, also zu 


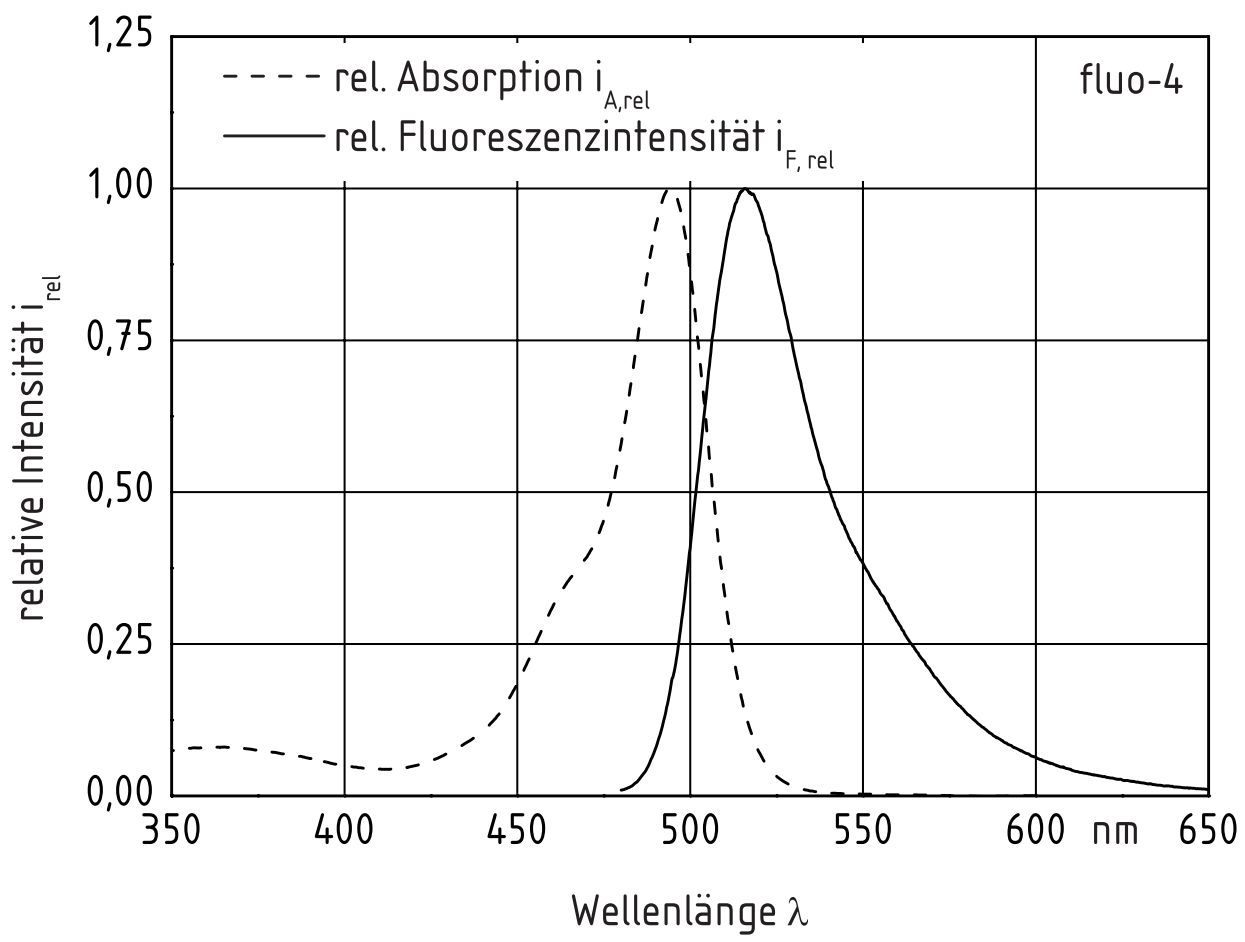

Bild 5.2: Normiertes Absorptions- und Emissionsspektrum des Fluoreszenzfarbstoffs fluo-4

größeren Wellenlängen hin verschoben. Da die Verteilung der Energieniveaus im Grundzustand und im ersten angeregten Zustand sehr ähnlich ist, sind die Energieunterschiede zwischen den Absorptionsbanden sehr ähnlich zu denen der Emissionsbanden. Das Fluoreszenzspektrum ist daher theoretisch ein ungefähres Spiegelbild des Absorptionsspektrums. Tatsächlich ist dies für die in Bild 5.2 dargestellten normierten Absorptionsund Emissionsspektren des in dieser Arbeit verwendeten Fluoreszenzfarbstoffs fluo-4 der Fall. Dabei ist das Emissionsspektrum zu längeren Wellenlängen hin verschoben. Aufgrund von Dämpfungseffekten und Wechselwirkungen mit dem Lösungsmittel weichen Absorptions- und Emissionsspektrum in der Praxis immer etwas von der Selbstähnlichkeit ab. Die Differenz der Wellenlänge zwischen dem Maximum der langwelligsten Absorptionsbande und dem Maximum der kurzwelligsten Fluoreszenzbande wird dabei als Stokes-Verschiebung bezeichnet.

\subsection{Messungen mit dem Fluoreszenzspektrometer}

Die oben beschriebene Fluoreszenzemission ist charakteristisch für einen Fluoreszenzfarbstoff. Die Kenntnis der spektroskopischen Eigenschaften sind notwendig für die Wahl eines geeigneten Farbstoffsystems, wie in Kapitel 6.1 erläutert wird. Im Folgenden wird deshalb kurz auf die Messung der Fluoreszenzspektren, dabei auftretende Messungenauigkeiten 
Tabelle 5.1: Einstellungen des Fluoreszenzspektrometers F-4500 (Hitachi)

\begin{tabular}{|l|c|}
\hline Anregungswellenlänge & $400-650 \mathrm{~nm}$ \\
Emissionswellenlänge & $450-750 \mathrm{~nm}$ \\
Schrittweite der Anregungs-/ Emissions- Wellenlänge & $5 \mathrm{~nm} / 5 \mathrm{~nm}$ \\
Bandbreite der Anregungs-/ Emissions- Wellenlänge & $10 \mathrm{~nm} / 10 \mathrm{~nm}$ \\
Spannung am Photomultiplier & $950 \mathrm{~V}$ \\
Scan-Geschwindigkeit & $12.000 \mathrm{~nm} / \mathrm{min}$ \\
\hline
\end{tabular}

und unterschiedliche Darstellungsformen eingegangen.

Zur Messung der charakteristischen Fluoreszenzemission der verwendeten Farbstoffe wird in dieser Arbeit das Fluoreszenzspektrometer F-4500 (Hitachi) verwendet. Als Lichtquelle dient eine Xenonlampe, die weißes Licht ausstrahlt. Mit Hilfe eines Gittermonochromators mit wählbarer Spaltbreite kann die Bandbreite der Anregungswellenlänge frei gewählt werden. Die Detektion des emittierten Lichts erfolgt senkrecht zum Anregungslicht über einen weiteren Gittermonochromator sowie einen Photomultiplier. Die Wellenlängen von Exzitation (Anregung) und Emission können somit unabhängig voneinander eingestellt werden. Für die Messung werden Küvetten aus Quarzglas verwendet, die mit den Farbstofflösungen befüllt und quer zum Anregungslicht in das Fluoreszenzspektrometer eingesetzt werden. Es werden die in Tabelle 5.1 angegebenen Einstellungen gewählt.

Für den Fluoreszenzfarbstoff fluo-4 ist in $\underline{\text { Bild } 5.3}$ beispielhaft die Intensität der Fluoreszenzemission in Abhängigkeit von der Anregungswellenlänge $\lambda_{e x}$ und der Emissionswellenlänge $\lambda_{e m}$ dargestellt. Die Fluoreszenzemission ist als Peak zu erkennen. Die Streuung des Anregungslichts ist als schmales Band relativ hoher Intensität um die Linie $\lambda_{e m}=\lambda_{e x} \mathrm{zu}$ erkennen. Um Störungen durch das Streulicht möglichst gering zu halten, müssen die Konzentration des Fluorophors und die Einstellungen des Fluoreszenzspektrometers so gewählt werden, dass die Intensität des detektierten Fluoreszenzlichts größer ist als die Intensität des gestreuten Lichts. Dabei muss ein Kompromiss zwischen hoher Empfindlichkeit der Messung und der spektralen Aufösung gefunden werden (s. Tabelle 5.1). Die Unregelmäßigkeiten in der Intensität des Streulichts sind auf die Interpolation zwischen den einzelnen Messwerten (in diesem Fall in 5nm-Schritten) zurückzuführen.

In einer Projektion auf die Ebene aus Anregungs- und Emissionswellenlänge wird, wie in Bild 5.4, die Intensität der Fluoreszenzemission als Kontur dargestellt. Aus Gründen der Übersichtlichkeit wird üblicherweise diese Darstellung gewählt. Die Intensität des Fluoreszenzlichts variiert um das Fluoreszenzmaximum bei einer Anregungswellenlänge von $\lambda_{e x} \approx 495 \mathrm{~nm}$ und einer Emissionswellenlänge von $\lambda_{e m} \approx 520 \mathrm{~nm}$.

Als Emissionsspektrum wird die Darstellung der Fluoreszenzintensität als Funktion der 


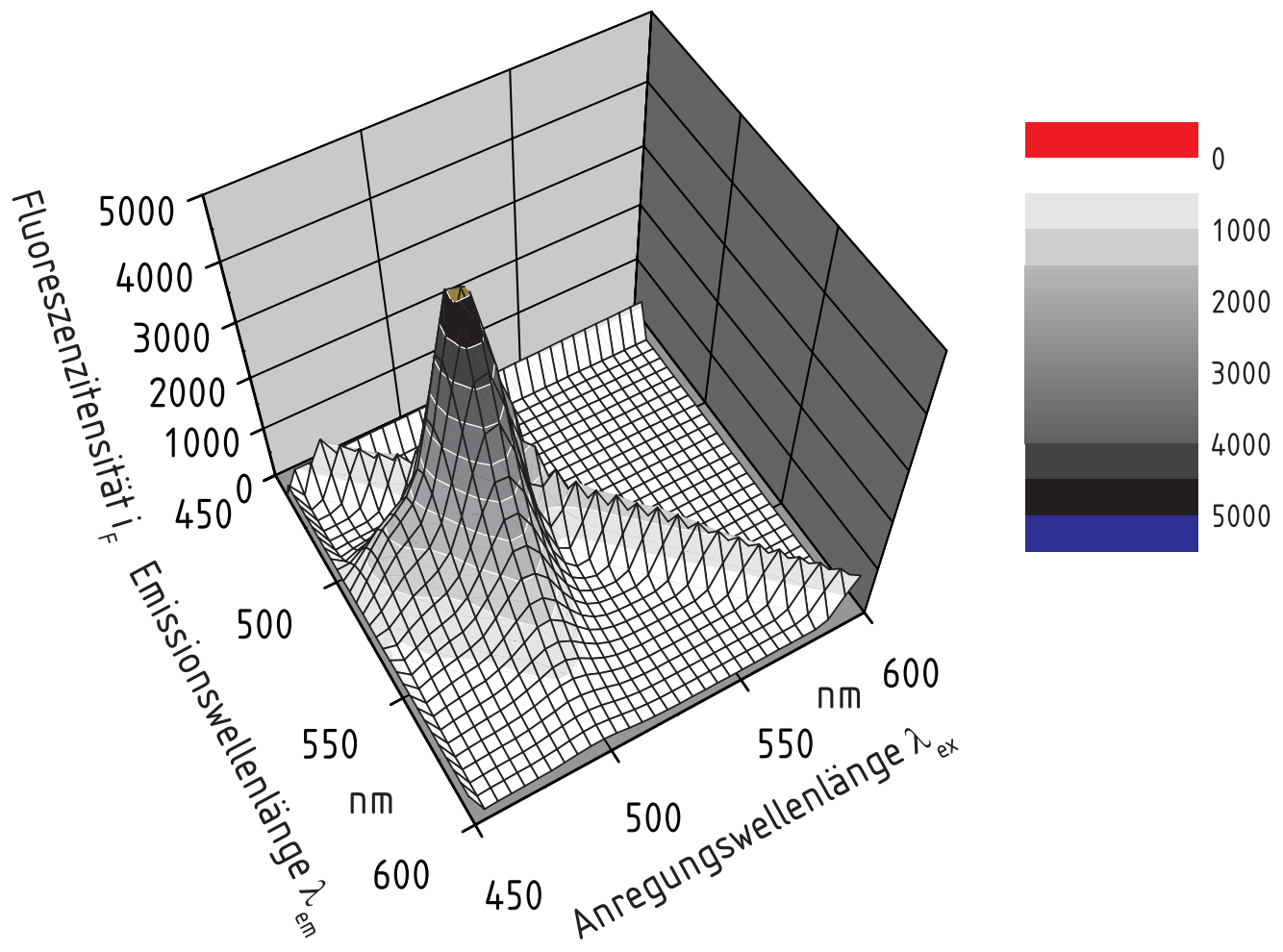

Bild 5.3: Fluoreszenzintensität in Abhängigkeit von Anregungs- und Emissionswellenlänge in dreidimensionaler Darstellung

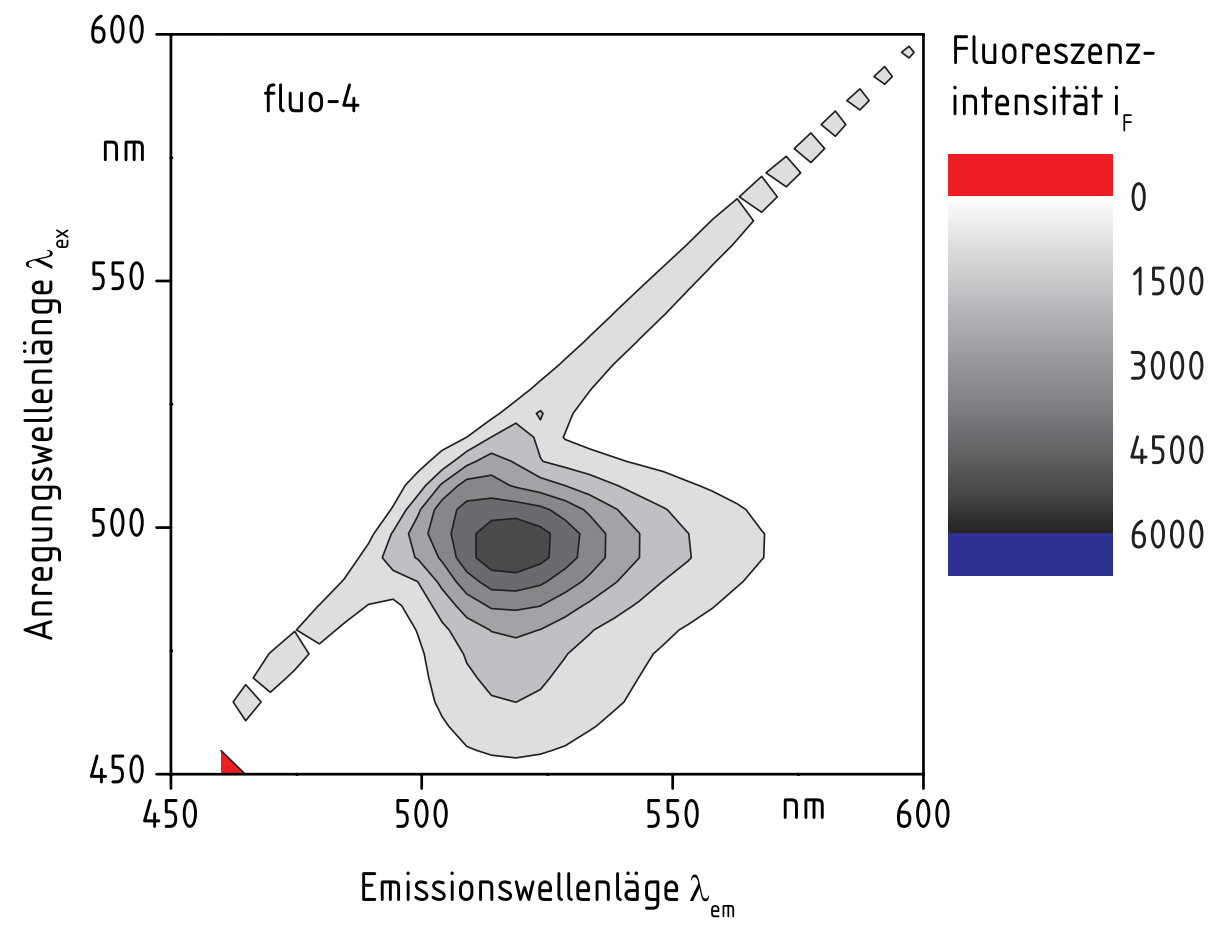

Bild 5.4: Fluoreszenzintensität in Abhängigkeit von Anregungs- und Emissionswellenlänge in zweidimensionaler Darstellung 


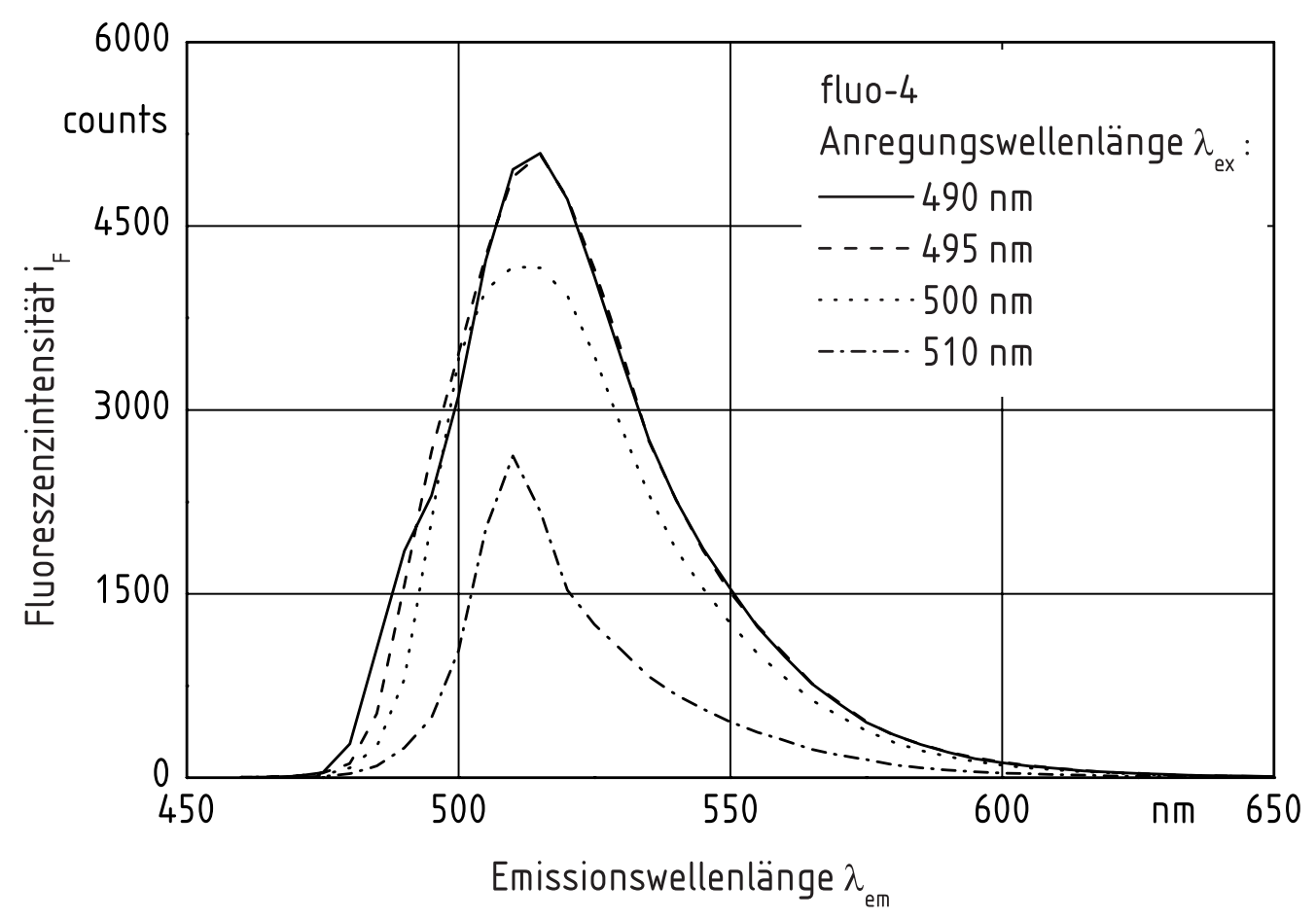

Bild 5.5: Fluoreszenzintensität in Abhängigkeit der Emissionswellenlänge für unterschiedliche Anregungswellenlängen

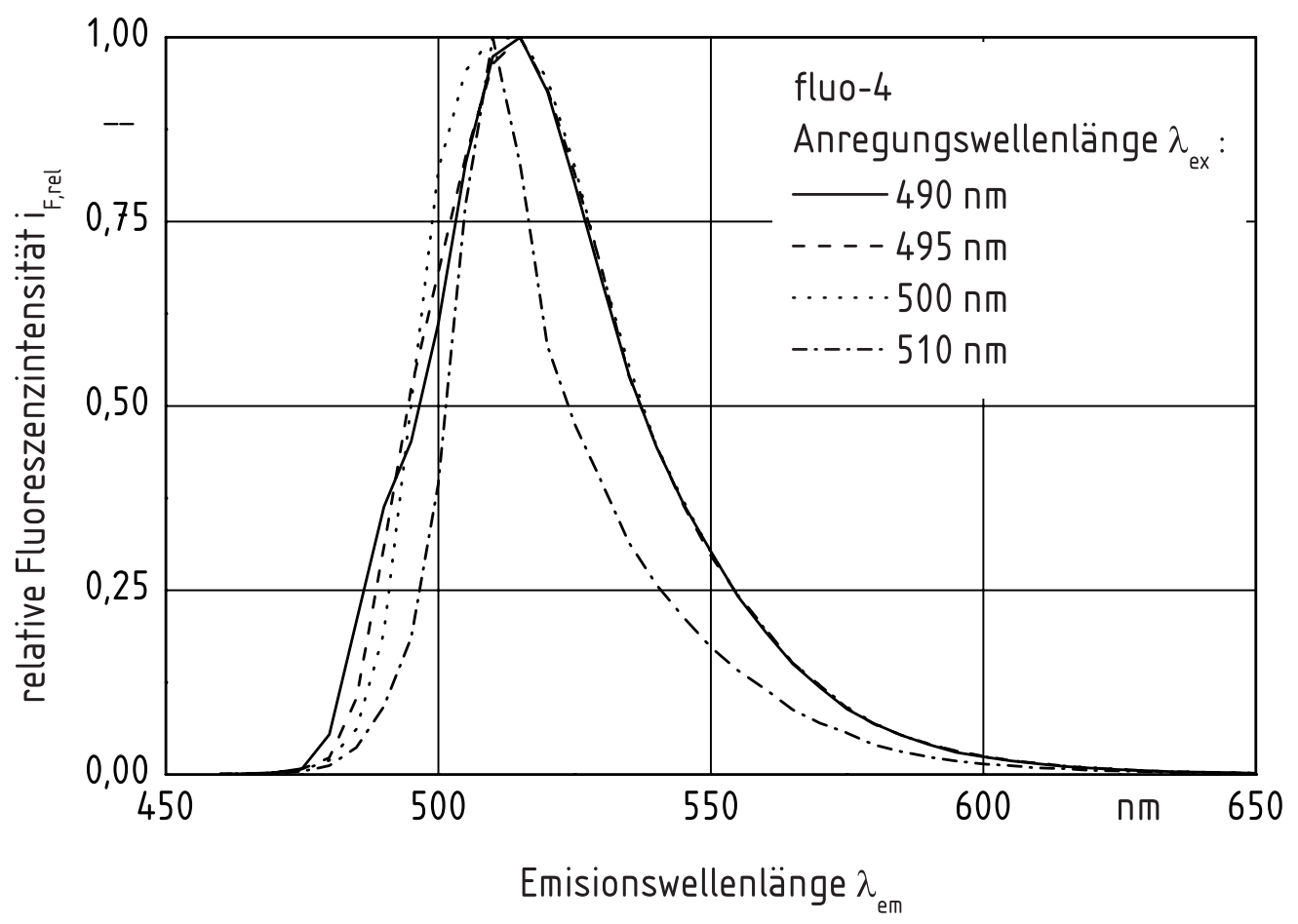

Bild 5.6: Relative Fluoreszenzintensität in Abhängigkeit der Emissionswellenlänge für unterschiedliche Anregungswellenlängen 
Wellenlänge des emittierten Lichts mit der Anregungswellenlänge als Parameter bezeichnet. Dieses kann anschaulich aus der dreidimensionalen Darstellung (Bild 5.3) gewonnen werden, indem ein Schnitt entlang der gewünschten Anregungswellenlänge ausgeführt wird. In Bild 5.5 sind die Emissionsspektren für vier unterschiedliche Anregungswellenlängen dargestellt. Da die Fluoreszenzemission immer durch den Übergang vom niedrigsten Energieniveau des ersten angeregten Zustands hervorgerufen wird, ist die Lage der Fluoreszenzbanden im Emissionsspektrums immer gleich und weitgehend unabhängig von der Wellenlänge des anregenden Lichts. Nur die Intensität der Fluoreszenz variiert mit der jeweiligen Anregungswellenlänge. Ein auf den jeweiligen Maximalwert der Fluoreszenzemission normiertes Emissionsspektrum

$$
i_{F, r e l}\left(\lambda_{e m}\right)=\frac{i_{F}\left(\lambda_{e m}\right)}{\max \left(i_{F}, \lambda_{e x}=\text { const }\right)}
$$

ist für alle Anregungswellenlängen näherungsweise gleich, s. Bild 5.6. Das Emissionsspektrum für die Anregungswellenlänge $\lambda_{e x}=510 \mathrm{~nm}$ zeigt hier die größte Abweichung, da sich die Anregungswellenlänge nur gering von der Haupt-Fluoreszenzwellenlänge von $\lambda_{\text {em }} \approx 520 \mathrm{~nm}$ unterscheidet und so die Störung der Messung durch das Streulicht besonders deutlich wird. Dieser Fehler bewirkt auch, dass Emissionswellenlängen kleiner als die Anregungswellenlänge gemessen werden. Dies kann aus energetischen Gründen, wie oben beschrieben, nicht der Fall sein. Interessiert also wirklich nur das Emissionsspektrum für eine feste Anregungswellenlänge, so liefert die direkte Messung eines sogenannten Linienspektrums bei eben dieser konstanten Wellenlänge genauere Ergebnisse.

Die insgesamt abgestrahlte Intensität der Fluoreszenzemission ist die Summe der Intensitäten über alle Wellenlängen

$$
I_{F}=\int_{\lambda=\lambda_{e x}}^{\lambda=\infty} i_{F}(\lambda) \mathrm{d} \lambda .
$$

Graphisch entspricht die Intensität also der Fläche unter dem Emissionsspektrum, was in Bild 5.7 durch die Schraffur angedeutet ist.

\subsection{Messungen mit dem Absorptionsspektrometer}

Die von einer Farbstofflösung absorbierte Strahlung entspricht der Differenz aus einfallender Strahlung $I_{0}$ und transmittierter Strahlung $I$. In Bild 5.8 ist ein Fluidelement der Dicke $\mathrm{d} x$ dargestellt, in das von Licht der Intensität $I(x)$ und der Wellenlänge $\lambda$ eintritt. Nach der infinitesimalen Weglänge $\mathrm{d} x$ ist die Intensität auf den Wert $I(x+\mathrm{d} x)=I(x)+\mathrm{d} I$ verringert, wobei die Änderung $\mathrm{d} I$ hier aufgrund der Absorption ein negatives Vorzeichen 


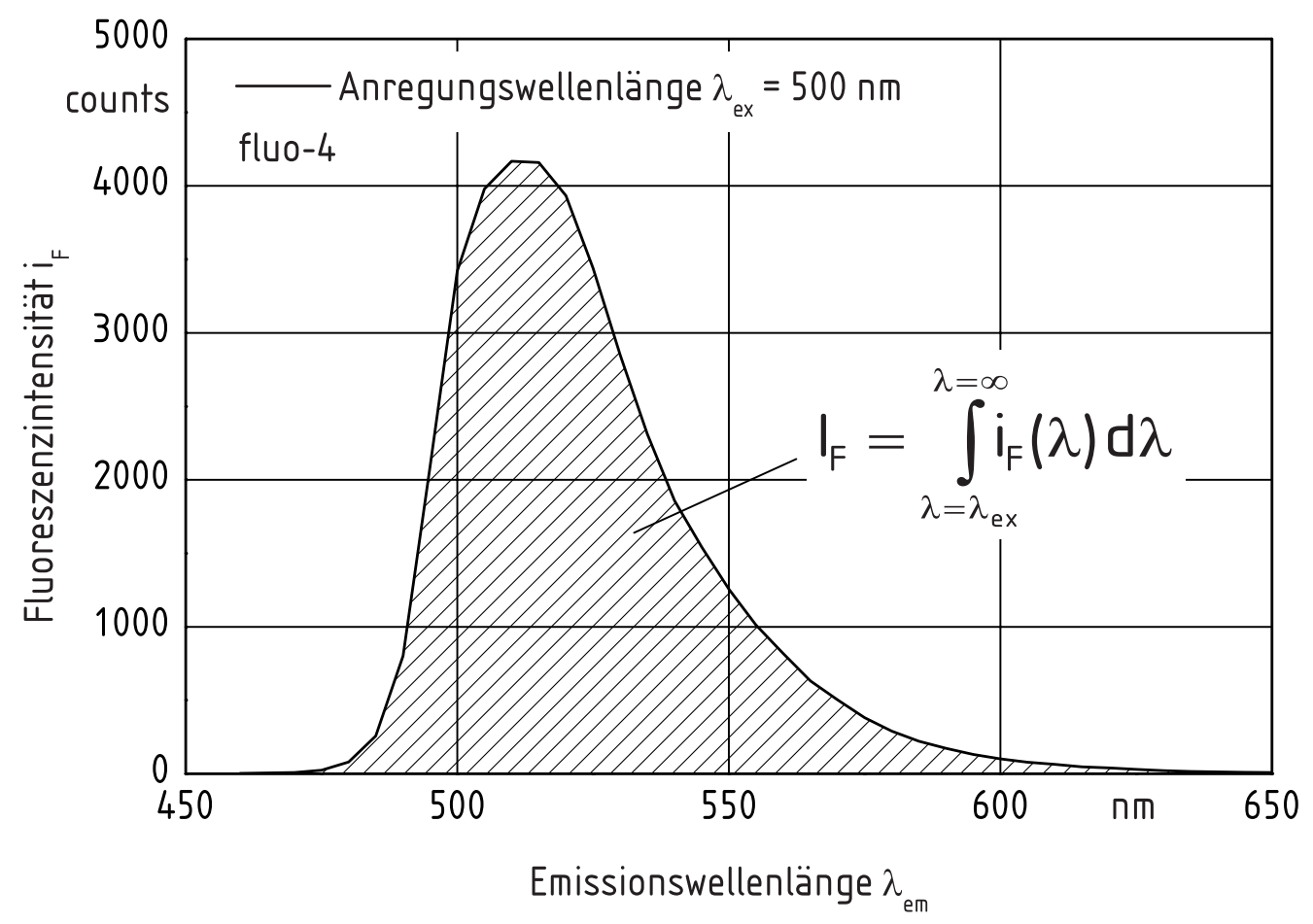

Bild 5.7: Fluoreszenzintensität in Abhängigkeit der Emissionswellenlänge und Gesamtfluoreszenzintensität $I_{F}$

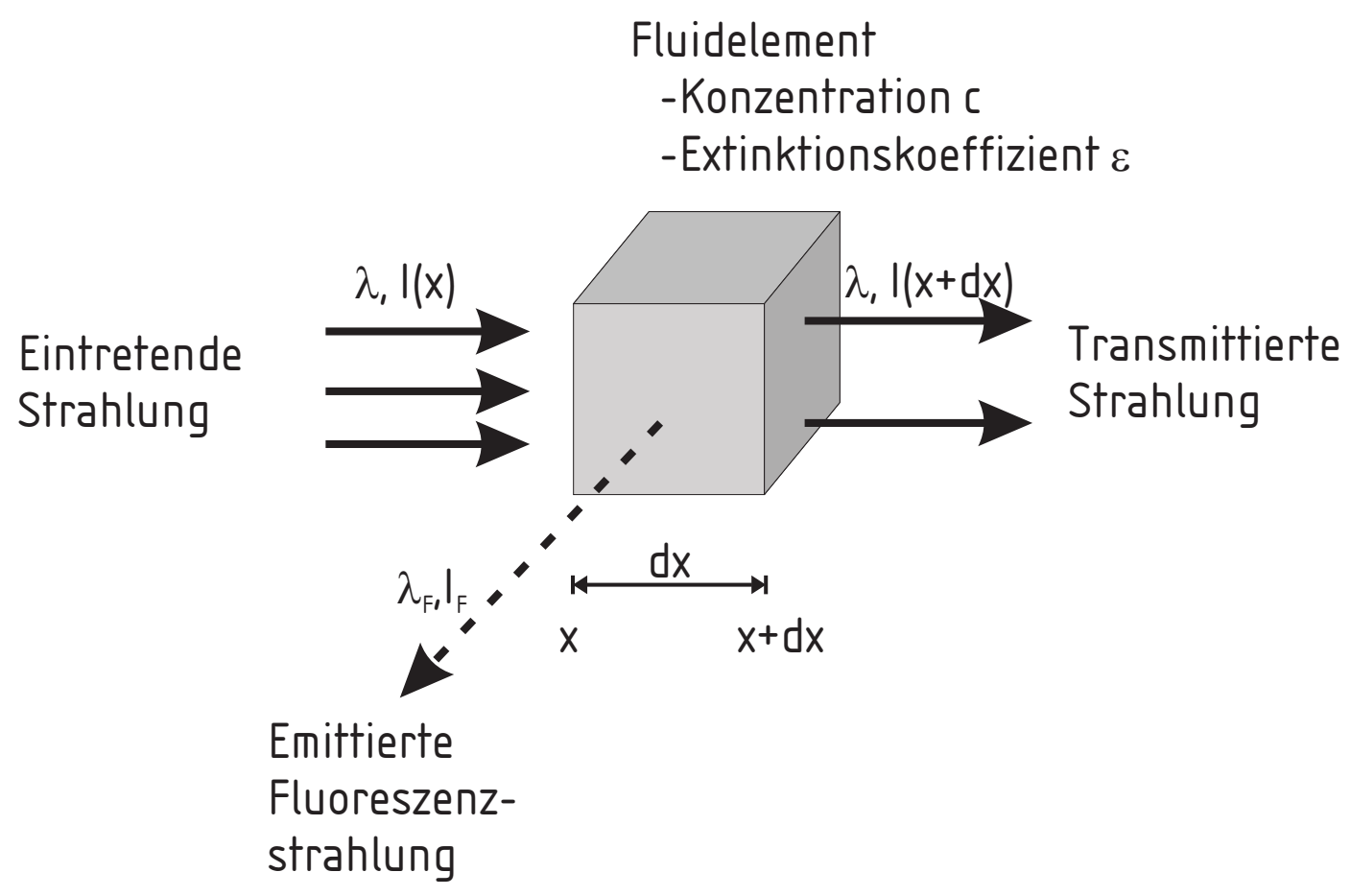

Bild 5.8: Schematische Darstellung der Absorption, Transmission und Fluoreszenzemission von Licht 
besitzt. Nach Lambert und Beer [111] ist die Intensität der auf einem Durchstrahlungsweg $\mathrm{d} x$ absorbierten Strahlung

$$
\mathrm{d} I=-I \varepsilon c \mathrm{~d} x
$$

proportional zu der Intensität der einfallenden Strahlung $I$, der Konzentration der homogenen Farbstoffösung $c$, der Weglänge $\mathrm{d} x$ und dem Extinktionskoeffizienten $\varepsilon$. Letzterer ist definiert als die optische Dichte einer ein-molaren Lösung eines Farbstoffs entlang eines Strahlwegs von $1 \mathrm{~cm}$. Er wird durch Kalibrieren bestimmt.

Die Integration von Gl. (5.3) über die Systemgrenzen

$$
\int_{I_{0}}^{I} \frac{\mathrm{d} I}{I}=-\int_{x=0}^{x=s} \varepsilon c \mathrm{~d} x
$$

liefert

$$
\ln \left(\frac{I_{0}}{I}\right)=\varepsilon s c .
$$

Daraus kann die dimensionslose Absorption hergeleitet werden [111]

$$
A=\log _{10}\left(\frac{I_{0}}{I}\right)=\varepsilon^{\prime} s c .
$$

Der molare Absorptionskoeffizient

$$
\varepsilon^{\prime}=\varepsilon \cdot \log _{10}(e) \approx 0,43429 \varepsilon,
$$

unterscheidet sich vom molaren Extinktionskoeffizienten $\varepsilon$ nur um den Faktor $\log _{10}(e)$, wobei $e$ die Eulersche Konstante ist. Gl. (5.6) dient der Auswertung von Messungen mit dem Absorptionsspektrometer, da sowohl $I$ als auch $I_{0}$ leicht zu messen sind. Für bekannte Werte der Durchstrahlungslänge $s$ (z.B. gegeben durch die verwendete Küvette) kann so entweder für bekannte Werte des Absorptionskoeffizienten bei einer bestimmten Wellenlänge $\lambda_{0}$ die Konzentration der Lösung

$$
c=\frac{A\left(\lambda_{0}\right)}{s \varepsilon^{\prime}\left(\lambda_{0}\right)}
$$

oder für eine bekannte Konzentration $c$ die Verteilung des molaren Absorptionskoeffizienten

$$
\varepsilon^{\prime}(\lambda)=\frac{A(\lambda)}{s c}
$$

berechnet werden. Letzteres ist in Bild 5.9 beispielhaft für den Farbstoff fluo-4 dargestellt. Die Kurven des normierten Absorptionsspektrums (s. Bild 5.2) und des normierten Spektrums des Absorptions- oder Extinktionskoeffizienten sind identisch.

Aus Gl. (5.5) kann die Intensität der transmittierten Strahlung

$$
I=I_{0} e^{-\varepsilon s c}
$$




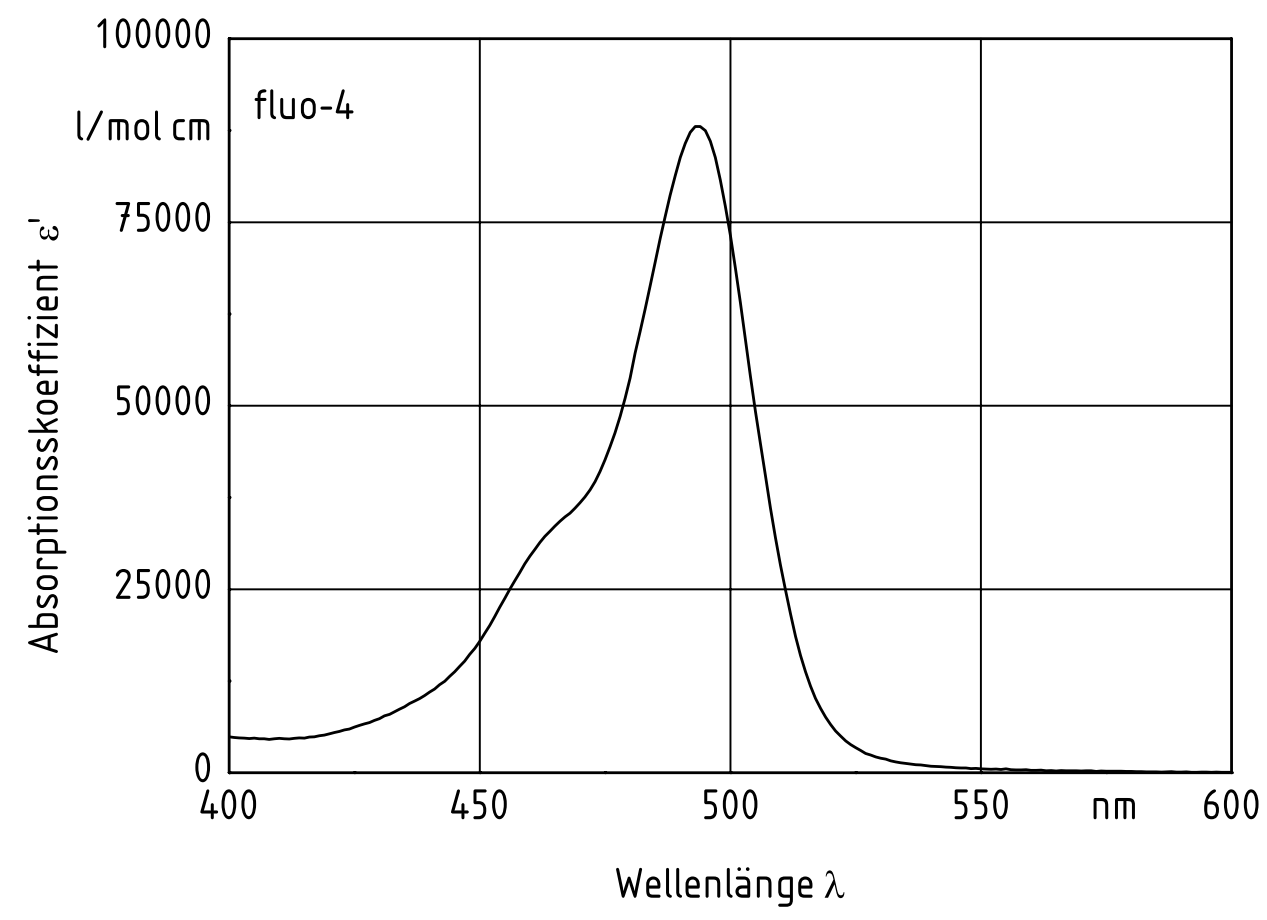

Bild 5.9: Absorptionskoeffizient in Abhängigkeit der Wellenlänge für den Fluoreszenzfarbstoff fluo-4

berechnet werden. Die Intensität der absorbierten Strahlung ist die Differenz zwischen eingestrahlter und transmittierter Strahlung

$$
I_{A}=I_{0}-I=I_{0}\left(1-e^{-\varepsilon s c}\right) .
$$

Für ein Gemisch aus mehreren Komponenten $j$ verallgemeinert sich der Exponent aus Gl. (5.11) zu

$$
\varepsilon s c=\sum_{j} \varepsilon_{j} c_{j} \cdot s,
$$

wobei $\varepsilon_{j}$ der Extinktionskoeffizient und $c_{j}$ die Konzentration der Komponente $j$ im Gemisch ist.

\subsection{Quantitative Auswertung}

Die Intensität der Fluoreszenzemission ist proportional zu der absorbierten Strahlung und verteilt sich auf den Wellenlängenbereich des Fluoreszenzspektrums. Die Quanteneffizienz $\Phi$ gibt an, welcher Teil der absorbierten Strahlung wieder als Fluoreszenz emittiert wird

$$
I_{F}=\Phi I_{A}=\Phi I_{0}\left(1-e^{-\varepsilon s c}\right)
$$


Die Quanteneffizienz nimmt Werte zwischen $\Phi=0$ für eine strahlungslose Energiedissipation und $\Phi=1$ für eine vollständige Energieabgabe in Form von Fluoreszenzstrahlung an.

Die Fluoreszenzemission ist der eingestrahlten Intensität $I_{0}$ direkt proportional. Bei Absorptionsmessungen wird hingegen laut Gl. (5.6) das Verhältnis $I / I_{0}$ gemessen. Die Empfindlichkeit dieser Methode ist durch den Umstand begrenzt, dass zwischen zwei großen, oft nur geringfügig unterschiedlichen Signalen zu unterscheiden ist. Bei der Fluoreszenzmessung wird hingegen gegen Dunkelheit, also gegen das Nullsignal gemessen. Dies ermöglicht eine optisch und elektronisch weitaus empfindlichere Messanordnung. In der Praxis ergeben sich dadurch Nachweisgrenzen von Konzentrationen von bis zu $10^{-12} \mathrm{~mol} / \mathrm{l}$ gegenüber $10^{-8} \mathrm{~mol} / \mathrm{l}$ für Absorptionsmessungen. Diese niedrige Nachweisgrenze wird nur in Spektrometern erreicht. Die während dieser Arbeit eingesetzte Messtechnik der Laserinduzierten Fluoreszenz kann Konzentrationen in der Größenordnung von $10^{-9} \mathrm{~mol} / \mathrm{l}$ detektieren.

Üblicherweise wird die exponentielle Abhängigkeit der Fluoreszenzintensität vom molaren Extinktionskoeffizienten $\varepsilon$, der Durchstrahlungslänge $s$ und der Konzentration $c$ vernachlässigt, so dass die linearisierte Form von Gl. (5.13) verwendet wird

$$
I_{F}=\Phi I_{0} \varepsilon s c
$$

Diese Vereinfachung ist allerdings nur für kleine Werte des Produkts (Esc) zulässig. Dann kann die Funktion $f(x)=e^{-\varepsilon s c}$ durch ihre Taylorreihe

$$
f(x)=f\left(x_{0}\right)+\frac{f^{\prime}\left(x_{0}\right)}{1 !}\left(x-x_{0}\right)+\frac{f^{\prime \prime}\left(x_{0}\right)}{2 !}\left(x-x_{0}\right)^{2}+\cdots+\frac{f^{(n)}\left(x_{0}\right)}{n !}\left(x-x_{0}\right)^{n}+R_{n}(x)
$$

um den Punkt $x_{0}=0$ angenähert werden. Werden die Terme höherer Ordnung in dieser Reihenentwicklung

$$
e^{-\varepsilon s c}=1-\varepsilon s c+\frac{(\varepsilon s c)^{2}}{2}-\frac{(\varepsilon s c)^{3}}{6}+\cdots+(-1)^{n} \frac{(\varepsilon s c)^{n}}{n !}
$$

vernachlässigt, so ist der Fehler der Näherung kleiner als 0,1\%, solange die Bedingung

$$
\varepsilon s c<0,05
$$

erfüllt ist. Für übliche Extinktionskoeffizienten und Durchstrahlungslängen ist diese Beziehung nur für kleine Konzentrationen erfüllt.

In der Praxis wird nur ein Teil der insgesamt ausgestrahlten Fluoreszenzintensität detektiert. Verluste ergeben sich z.B. durch den Beobachtungswinkel des Detektors, da im allgemeinen nur die senkrecht zum Lichtschnitt emittierte Strahlung gemessen wird. Außerdem wird die Intensität durch vorgeschaltete Linsen, Spiegel und evtl. durch nur für 
bestimmte Wellenlängen durchlässige optische Filter reduziert. Gleichung (5.14) wird daher um den Parameter $K$ erweitert, der alle Verluste des Messsystems berücksichtigt

$$
I_{F, \text { detekt }}=K \Phi I_{0} \varepsilon s c .
$$

Im Allgemeinen wird der Parameter $K$ nicht ermittelt, da auch die Werte für $I_{0}, \Phi$ und $\varepsilon$ nicht ausreichend genau bekannt sind. Vielmehr wird ein Messsystem üblicherweise so kalibriert, dass der Proportionalitätsfaktor

$$
\begin{aligned}
m & =K \Phi I_{0} \varepsilon s \\
& =\frac{I_{F, \text { detekt }}}{c}
\end{aligned}
$$

zwischen gemessener Fluoreszenzintensität $I_{F, \text { detekt }}$ und Konzentration des Fluoreszenzfarbstoffs $c$ bestimmt wird, der somit bereits alle Verluste berücksichtigt. Voraussetzung für diese Vereinfachung ist, dass der Proportionalitätsfaktor $m$ während der Messungen konstant ist. Problematisch sind dabei insbesondere Schwankungen der Intensität der Lasers $I_{0}$. Dadurch bedingte Fehler können jedoch durch eine zeitgleiche Messung der Laserleistung korrigiert werden, wie später in Kapitel 8.2.2 gezeigt wird. 


\section{Eingesetzte Flüssigkeiten und Farbstoffe}

\subsection{Kriterien für die Auswahl des Farbstoffsystems}

Die verwendeten Fluoreszenzfarbstoffe werden als Gemische verwendet und sollten eine Reihe von Voraussetzungen erfüllen. Im Folgenden werden diese Voraussetzungen aufgezählt und bewertet.

$\mathrm{Zu}$ den nicht notwendigen aber aus praktischen Gründen wünschenswerten Voraussetzungen zählt, dass die Farbstoffe

- wasserlöslich,

- ungiftig und

- leicht entsorgbar sein sollten.

Unverzichtbar ist, dass

- nur einer der beiden Farbstoffe eine chemische Reaktion mit einer im Behälter vorgelegten Komponente eingeht, wobei seine Fluoreszenzeigenschaften verändert werden.

- die Zeitkonstante der chemischen Reaktion viel kleiner ist als die der Vermischung (Mischungshemmung anstatt Reaktionshemmung). Die chemische Reaktion muss also geeignet sein, den diffusiven Stofftransport auf mikroskopischer Ebene ohne Zeitverzug sichtbar zu machen.

- die Farbstoffe geeignete spektroskopische Eigenschaften aufweisen.

Der letzte Punkt ist wesentlich für die Auswahl des Farbstoffsystems und birgt mehrere Eigenschaften, die im Folgenden erläutert werden. Dazu gehört, dass beide Farbstoffe mit der gleichen Wellenlänge anregbar sein sollen. Voraussetzung ist dann, dass die Absorptionsspektren der beiden Farbstoffe überlappen. Um die Handhabung zu erleichtern und die Verletzungsgefahr durch das Laserlicht für den Anwender gering zu halten, ist die Anregungswellenlänge vorzugsweise im sichtbaren Bereich zu wählen.

Weiterhin sind sogenannte spektrale Konflikte möglichst auszuschließen. Darunter versteht man das Überlappen von Absorptions- und/ oder Emissionsspektren. Überlappt das Absorptionsspektrum eines Farbstoffs mit seinem eigenen Emissionsspektrum, so 


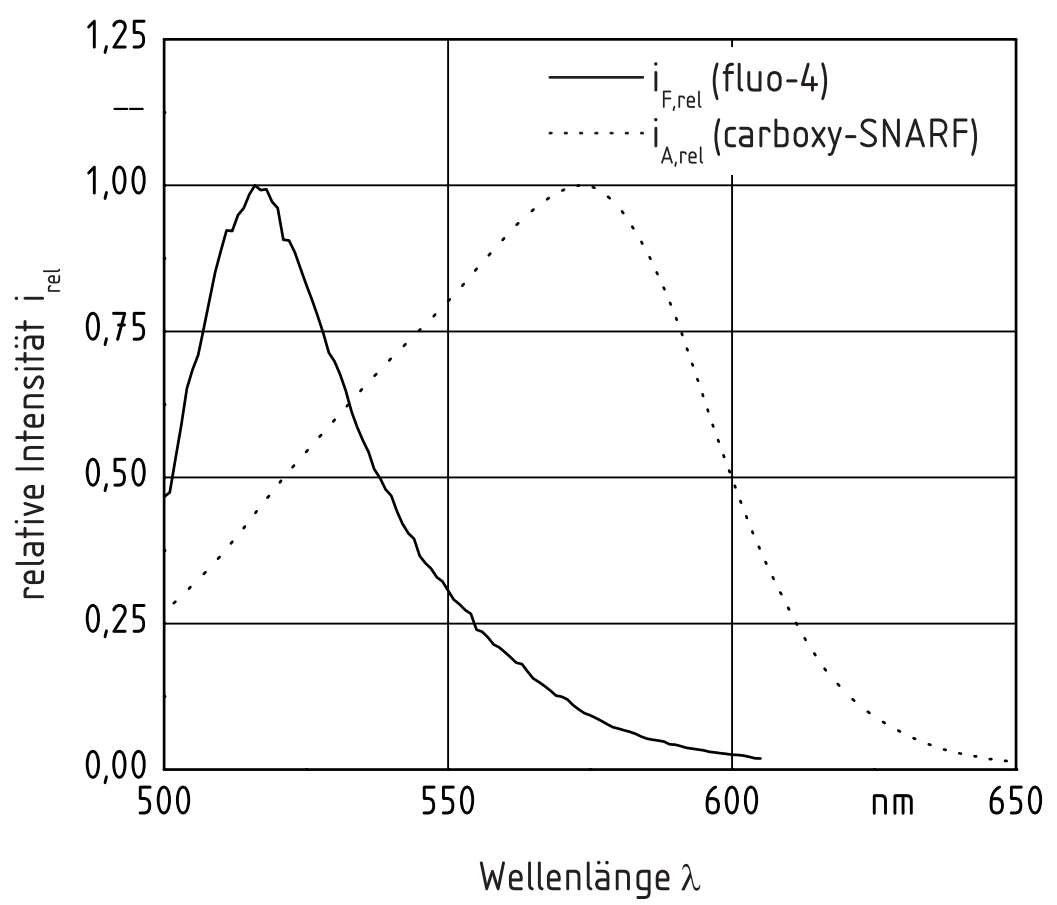

Bild 6.1: Das Emissionsspektrum von fluo-4 und das Absorptionsspektrum von carboxy-SNARF überlappen

spricht man von Selbst- oder Reabsorption. Diese tritt relativ häufig auf, und es gibt keine Messmethode, mit der das Emissionsspektrum getrennt von der Selbstabsorption gemessen werden kann. Allerdings ist der Fehler nur bei großen Konzentrationen bedeutsam, denn mit zunehmender Konzentration steigt die Wahrscheinlichkeit, dass die emittierte Strahlung eines Moleküls von einem anderen reabsorbiert wird. Für den Gültigkeitsbereich der linearen Beziehung zwischen Fluoreszenzemission und Konzentration laut Gl. (5.14) ist der Fehler der Selbstabsorption vernachlässigbar. Es sollten auch deshalb nur kleine Konzentrationen gemäß Gl. (5.17) verwendet werden. Der Arbeitsbereich für das in dieser Arbeit verwendete Farbstoff- und Messsystem wird in Kapitel 8.2.4 festgelegt.

Ein spektraler Konflikt zweiter Ordnung liegt vor, wenn das Absorptionsspektrum eines Farbstoffs mit dem Emissionsspektrum des zweiten Farbstoffs überlappt, wie in Bild 6.1 gezeigt. Dann wird der zweite Farbstoff zusätzlich zum monochromatischen Laserlicht von der Fluoreszenzemission des ersten Farbstoffs angeregt. Dadurch werden beide Emissionsspektren verfälscht, wie in Bild 6.2 schematisch dargestellt ist. Eine Farbstofflösung aus zwei Farbstoffen mit den Konzentrationen $c_{1}$ und $c_{2}$ und den molaren Extinktionskoeffizienten $\varepsilon_{1}$ und $\varepsilon_{2}$ wird mit Laserlicht der Wellenlänge $\lambda_{0}$ bestrahlt. Entlang des Weges $s$ wird die Strahlung des Lasers absorbiert und die Fluoreszenzintensitäten

$$
\begin{aligned}
& I_{F 1}=\Phi_{1} I_{0} s \varepsilon_{1} c_{1} \text { und } \\
& I_{F 2}=\Phi_{2} I_{0} s \varepsilon_{2} c_{2}
\end{aligned}
$$




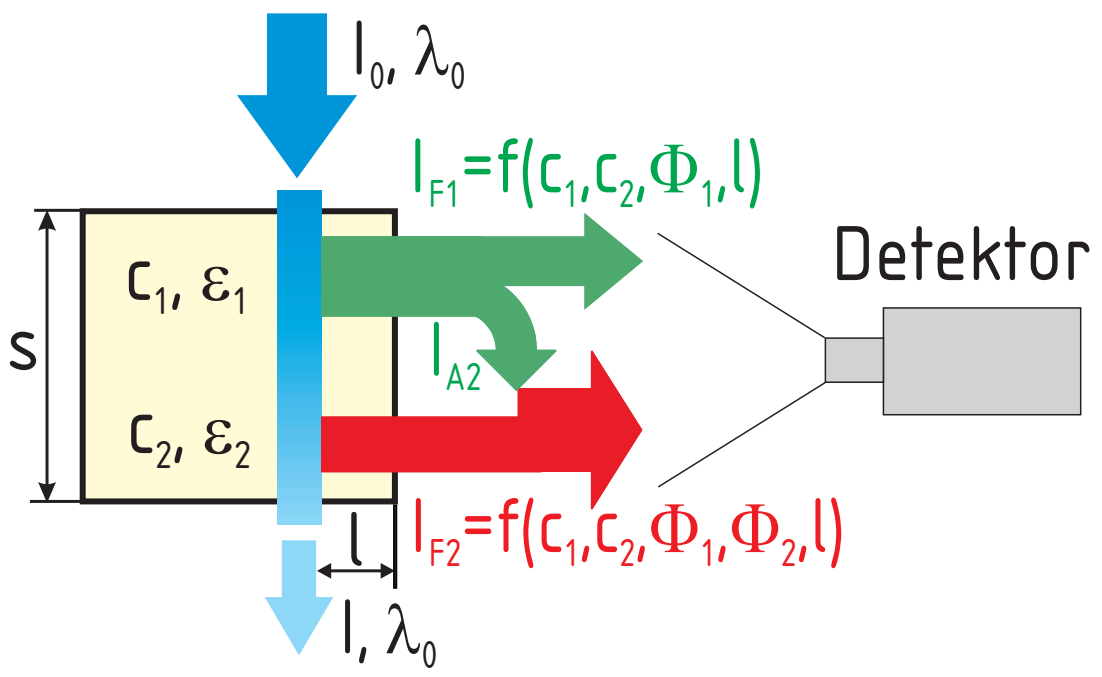

Bild 6.2: Schematische Darstellung der Reabsorption des Fluoreszenzlichts von fluo-4(1) durch carboxy-SNARF $(2)$

emittiert. Bevor das Fluoreszenzlicht detektiert wird, muss es den Weg $l$ in der Farbstofflösung passieren. Dabei absorbiert die Farbstofflösung 2 Teile des von Farbstoff 1 emittierten Fluoreszenzlichts, wodurch die Fluoreszenzintensität des Farbstoffs 1

$$
I_{F 1}^{*}=I_{F 1}-I_{A 2}
$$

um den Anteil $I_{A 2}$ sinkt, während die Fluoreszenzintensität des Farbstoffs 2

$$
I_{F 2}^{*}=I_{F 2}+I_{F, A 2}
$$

zunimmt. Die Intensität $I_{A 2}$ ist der Anteil des von Farbstoff 1 emittierten Fluoreszenzlichts, der von Farbstoff 2 absorbiert werden kann, während

$$
I_{F, A 2}=\Phi_{2} I_{A 2}
$$

der Anteil der absorbierten Strahlung ist, die von Farbstoff 2 wieder als Fluoreszenzlicht emittiert wird. Die absorbierte Strahlung $I_{A 2}$ berechnet sich zu

$$
I_{A 2}=I_{F 1}\left(1-e^{-\varepsilon_{2} c_{2} l}\right)
$$

mit dem Fluoreszenzlicht $I_{F 1}$ als einstrahlende Intensität. Da die Fluoreszenzintensität $i_{F 1}$ und der Extinktionskoeffizient $\varepsilon_{2}$ Funktionen der Wellenlänge $\lambda$ sind, gilt dies auch für die absorbierte Strahlung $i_{A 2}$. Nach Gl. (5.2) ist die insgesamt absorbierte Intensität das Integral über alle Wellenlängen

$$
I_{A 2}=\int_{\lambda=\lambda_{e x}}^{\lambda=\infty} i_{A 2}(\lambda) \mathrm{d} \lambda .
$$


Wird das reduzierte Fluoreszenzsignal $i_{F 1}^{*}$ auf das ursprüngliche Fluoreszenzsignal $i_{F 1}$ bezogen, so gibt das Verhältnis

$$
i_{F 1, \text { norm }}^{*}=\frac{i_{F 1}-i_{A 2}}{i_{F 1}}=e^{-\varepsilon_{2}(\lambda) c_{2} l},
$$

an, welcher Anteil des ursprünglichen Fluoreszenzsignals auch nach der Reabsorption detektiert werden kann. Ein Verhältnis von $i_{F 1, n o r m}^{*}=1$ beschreibt demnach den Fall ohne Reabsorption. In Bild 6.3 ist das Produkt aus $i_{F 1, n o r m}^{*}$ und der relativen Fluoreszenzintensität $i_{F 1, \text { rel }}$ über der Wellenlänge in Abhängigkeit von der Farbstoffkonzentration $c_{2}$ und der Lauflänge der Strahlung in der Lösung $l$ dargestellt. Der Anteil der absorbierten Strahlung hängt stark von der Konzentration des absorbierenden Farbstoffs und der Lauflänge in der Lösung ab. In Kapitel 8.2.4 wird für die verwendete Abmessung des Rührgefäßes und die verwendeten Farbstoffkonzentrationen die Absorption abgeschätzt. Ist der Fehler vernachlässigbar, so kann auch der Fehler beim Messen der Fluoreszenzemission des Farbstoffs 2 vernachlässigt werden, denn nach Gl. (6.5) wird nur ein Teil der absorbierten Strahlung wieder als Fluoreszenz emittiert.

Ein weiteres Problem tritt beim Auswerten der Messergebnisse auf, wenn sich die Emissionsspektren beider Farbstoffe überlappen. Dann ist die eindeutige Zuordnung der Fluoreszenzemission zu einem Farbstoff nur eingeschränkt möglich. Wässrige Lösungen weisen aufgrund von Wechselwirkungen der Farbstoffmoleküle mit dem Lösungsmittel zumeist ein breites Absorptions- und Emissionsspektrum auf, so dass überlappende Spektren kaum zu vermeiden sind. Dies gilt insbesondere für Farbstoffe, deren Absorptions-

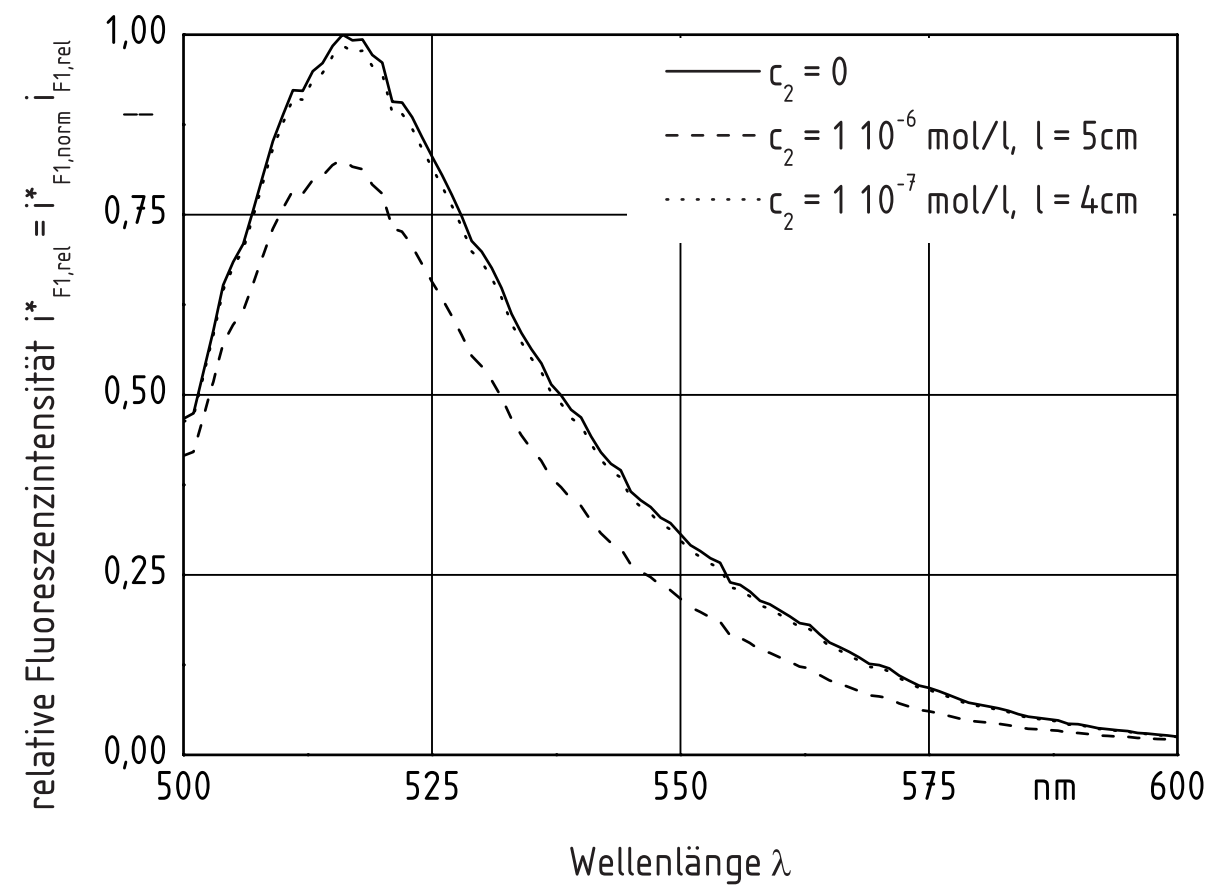

Bild 6.3: Fluoreszenzintensität von fluo-4 und reabsorbierter Anteil von carboxy-SNARF für unterschiedliche Farbstoffkonzentrationen und Durchstrahlungslängen 
spektren überlappen und die somit als Farbstoffsystem wie oben definiert einsetzbar sind. Die Auswertung der Messergebnisse kann nur eindeutig erfolgen, wenn mit Hilfe geeigneter optischer Filter die Teile des jeweiligen Emissionsspektrums herausgefiltert werden, in denen die Überlagerung vernachlässigbar ist. Dazu müssen die charakteristischen Fluoreszenzwellenlängen der beiden Farbstoffe ausreichend weit auseinander liegen. In Kapitel 8.2.4 werden die Transmissionseigenschaften der in dieser Arbeit verwendeten Filter sowie Korrekturmöglichkeiten aufgrund nicht-idealer Trennwirkung erläutert.

\subsection{Farbstoffe und Lösungsmittel}

Das verwendete Fluoreszenzfarbstoffsystem wurde entsprechend der oben genannten Kriterien ausgewählt und besteht aus dem reagierenden Farbstoff fluo-4 (Index 1) und dem inerten Farbstoff carboxy-SNARF (Index 2) (beide Firma Molecular Probes Inc.). Beide Farbstoffe sind gut in Wasser löslich, ungiftig, und die Lösungen sind als Hausmüll entsorgbar.

Der Farbstoff fluo-4 ist ein Indikator für Calciumionen. In einer Lösung, die frei von Calciumionen ist, ist nur eine geringe Hintergrundfluoreszenz nachweisbar. Mit zunehmender Konzentration an freien Calciumionen in der Lösung geht das Farbstoffmolekül mit den Calciumionen einen ungerichteten Koordinationskomplex ein, wodurch die Fluoreszenzintensität bis auf einen Maximalwert ansteigt. Die Komplexreaktion verläuft nach dem Schema

$$
\mathrm{Ca}^{2+}+\mathrm{A} \underset{k_{A^{-}}}{\stackrel{k_{A^{+}}}{\leftrightarrows}} \mathrm{CaA}
$$

wobei A das Farbstoffmolekül fluo-4 repräsentiert und $k_{A^{+}}$und $k_{A^{-}}$die Geschwindigkeitskonstanten der Komplexbildung bzw. des Zerfalls sind. Die Komplexbildung ist eine Reaktion 2. Ordnung und geschieht quasi spontan mit einer Geschwindigkeitskonstanten [112], [113] von etwa

$$
k_{A^{+}} \approx 10^{9} \frac{\mathrm{l}}{\mathrm{mol} \mathrm{s}}
$$

während der Zerfall eine Reaktion 1. Ordnung und deutlich langsamer mit

$$
k_{A^{-}} \approx 370 \mathrm{~s}^{-1}
$$

abläuft. Die Bedingung einer sehr schellen chemischen Reaktion gemäß der Kriterien in Kapitel 6.1 ist somit erfüllt. In der Literatur (s. Kapitel 3.1.2) werden überwiegend SäureBase-Reaktionen als spontane Reaktionen verwendet. Als Maß für die Vermischung auf mikroskopischer Ebene wird das Feld des pH-Werts gemessen. Säure-Base-Reaktionen haben den Vorteil, dass sie reversibel und kostengünstig sind. Als Nachweis für einen diffusiven Transport der miteinander zu vermischenden Reaktionspartner ist diese Reaktion 

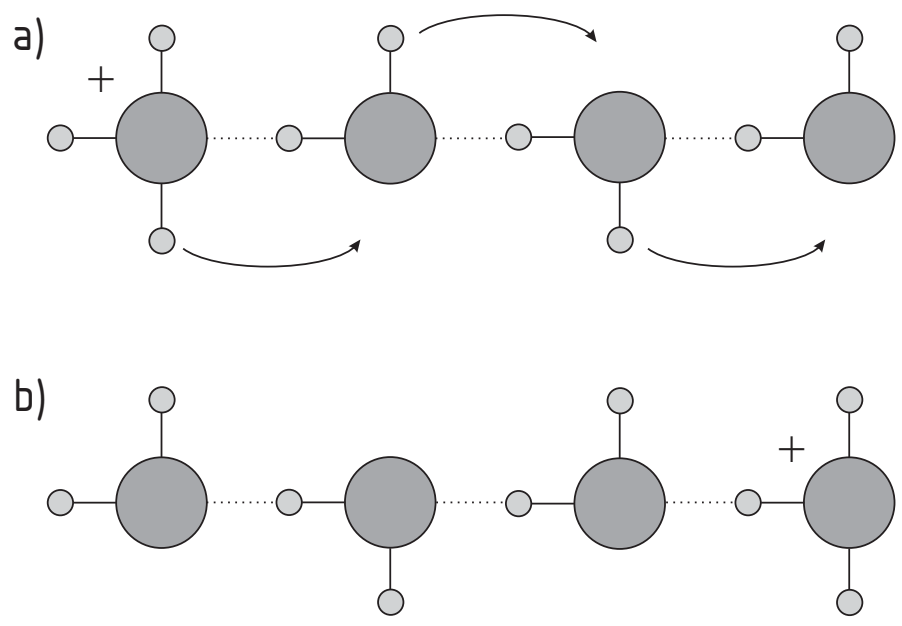

\section{Sauerstoff (O) $\bigcirc$ Wasserstoff $(H)$}

Bild 6.4: Schematische Darstellung des Grotthus'schen Transportmechanismus'

allerdings nur bedingt geeignet. Der Grotthus'sche Transportmechanismus [234] besagt nämlich, dass ein Ladungstransport von Protonen in einer Hydrathülle ohne einen Platzwechsel der Ionen erfolgen kann. Schematisch ist dieser Transportmechanismus in Bild 6.4 dargestellt: nicht einzelne hochsolvatisierte Protonen wandern durch die Lösung, sondern der Protonen-Transport erfolgt im Wesentlichen durch Umlagerung von Bindungen in einer langen Kette von (über H-Brücken assoziierten) Wassermolekülen. Demnach wird der lokale pH-Wert durch die Schnelligkeit bestimmt, mit der sich die Wassermoleküle umorientieren können, nicht aber durch den diffusiven Transport von Molekülen. In dieser Arbeit wird deshalb auf die Verwendung von pH-Wert-abhängigen Fluoreszenzfarbstoffen als reaktive Komponente verzichtet und statt dessen ein Reaktionssystem gewählt, das tatsächlich transportgehemmt ist. Das System aus fluo-4 und $\mathrm{Ca}^{2+}$ ist durch die Diffusion des viel kleineren Calciumions bestimmt [114]. Für wässrige Lösungen wird der Diffusionskoeffizient der Calciumionen mit

$$
D_{\mathrm{Ca}^{2+}} \approx 1,6 \cdot 10^{-9} \frac{\mathrm{m}^{2}}{\mathrm{~s}}
$$

angegeben. Peev und Nikolova [115] haben gezeigt, dass die Diffusionskoeffizienten kleiner Moleküle in Carboxymethylzellulose- Lösungen nur gering von ihren Diffusionskoeffizienten in Wasser abweichen, da sich die kleinen Moleküle ungehindert durch die Struktur der Makromoleküle bewegen können. Es wird daher der Diffusionskoeffizient in Wasser verwendet.

In Bild 6.5 sind die Absorptions- und Emissionsspektren von fluo-4 und carboxy-SNARF dargestellt. Die Absorptionsspektren überlappen in einem Wellenlängenbereich um 500 nm, so dass beide Farbstoffe gleichzeitig mit Licht in diesem Wellenlängenbereich an- 


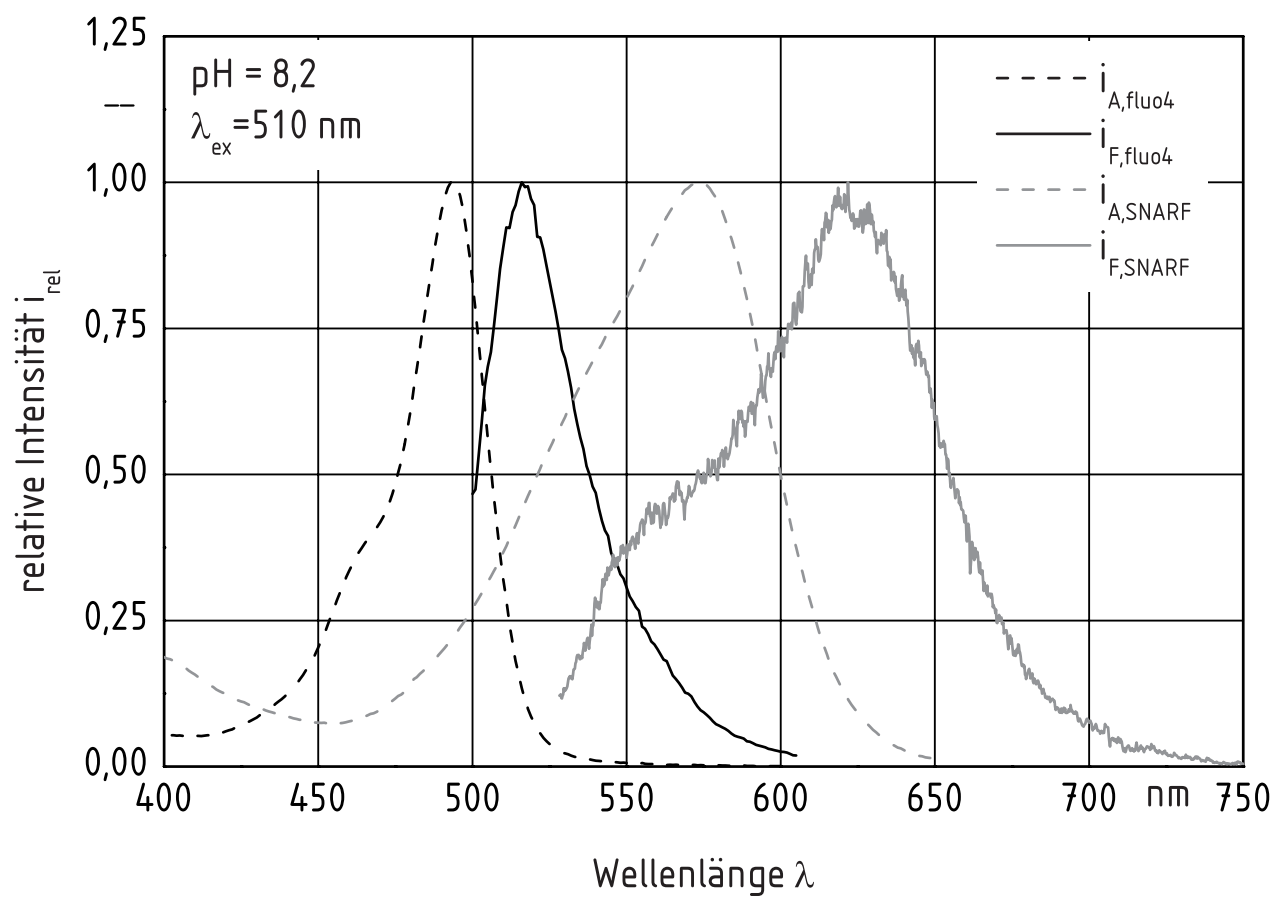

Bild 6.5: Absorptions- und Emissionsspektren von fluo-4 und carboxy-SNARF

regbar sind. Auch die Emissionsspektren überlappen in einem Bereich um $550 \mathrm{~nm}$. Eine eindeutige Zuordnung der Fluoreszenzsignale zu dem jeweiligen Farbstoff ist aber durch die Wahl von geeigneten optischen Filtern möglich, wie den Emissionsspektren in Bild 6.6 zu entnehmen ist. Der Bandpaßfilter BP523 mit der vollen Halbwertsbreite von 10nm (s. Bild 6.7) ist ein Interferenzfilter und nur für das Fluoreszenzlicht von fluo-4 durchlässig. Der Kantenfilter RG645 (s. Bild 6.8) ist ein Farbglasfilter, der erst ab einer Wellenlänge von etwa $625 \mathrm{~nm}$ durchlässig wird und somit nahezu ausschließlich das von carboxySNARF emittierte Fluoreszenzlicht transmittiert. Allerdings ist ein Farbglasfilter qualitativ einem Interferenzfilter deutlich unterlegen, so dass ein geringer Prozentsatz des von fluo-4 emittierten Fluoreszenzlichts auch durch den Filter RG645 detektiert werden kann. Auf dadurch entstehende Messfehler und deren Korrektur wird in Kapitel 8.2.4 näher eingegangen.

Für beide Farbstoffe gilt, dass jeweils ihre Absorptions- und Emissionsspektren überlappen, so dass eine Selbstabsorption möglich ist. In Kapitel 8.2.4 wird für beide Farbstoffe der Konzentrationsbereich festgelegt, in dem die lineare Abhängigkeit der Fluoreszenzemission von der Konzentration für das verwendete Messsystem gewährleistet ist.

Ein weiterer spektraler Konflikt, der in Kapitel 6.1 erwähnt wird, ist die Überlappung des Emissionsspektrums eines Farbstoffs mit dem Absorptionsspektrum des anderen Farbstoffs. Tatsächlich absorbiert carboxy-SNARF Teile des von fluo-4 emittierten Fluoreszenzlichts (s. Bild 6.5), so dass der dadurch entstehende Messfehler abgeschätzt werden 


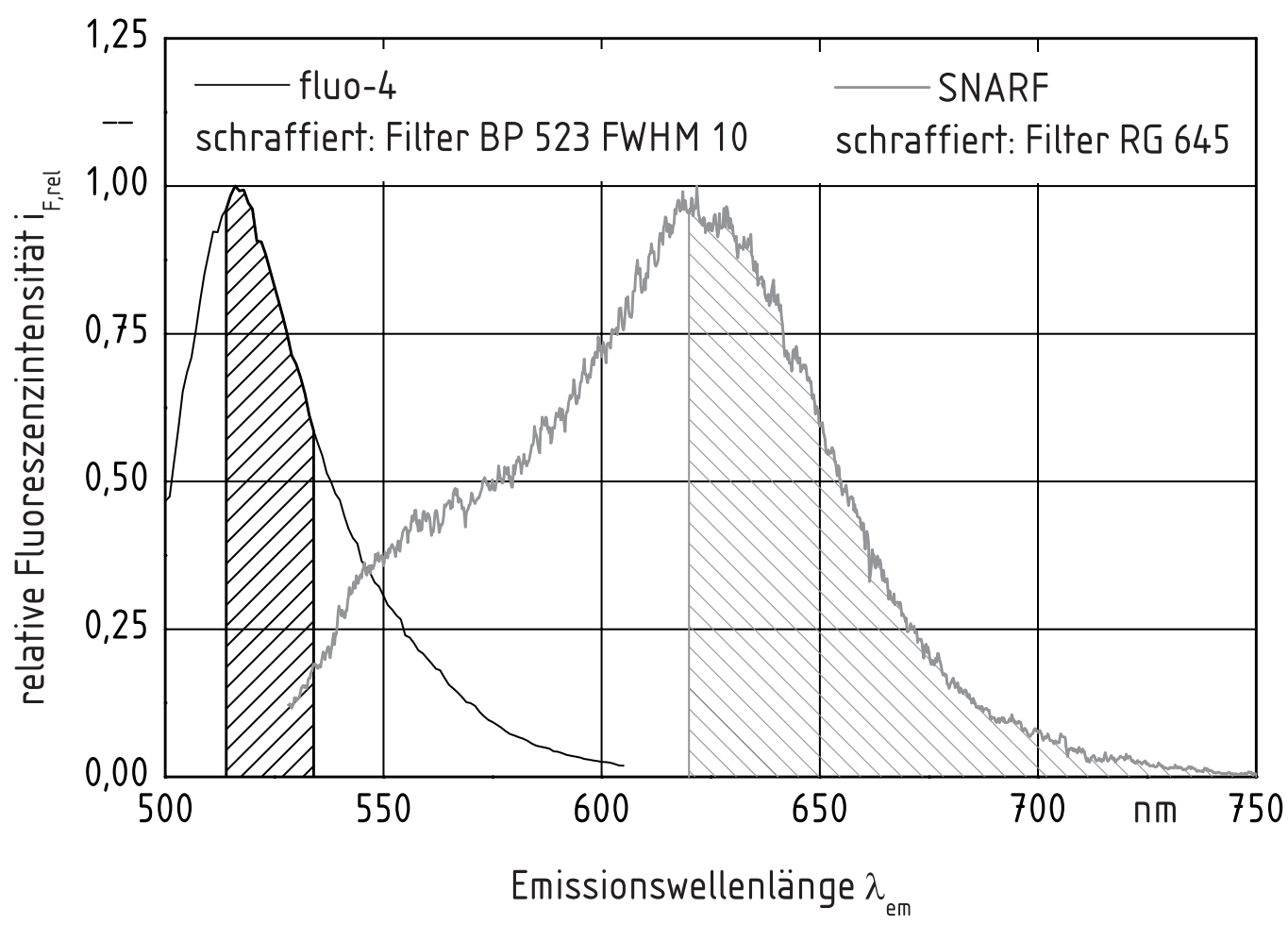

Bild 6.6: Emissionsspektren von fluo-4 und carboxy-SNARF und Transmissionsbereich der verwendeten optischen Filter

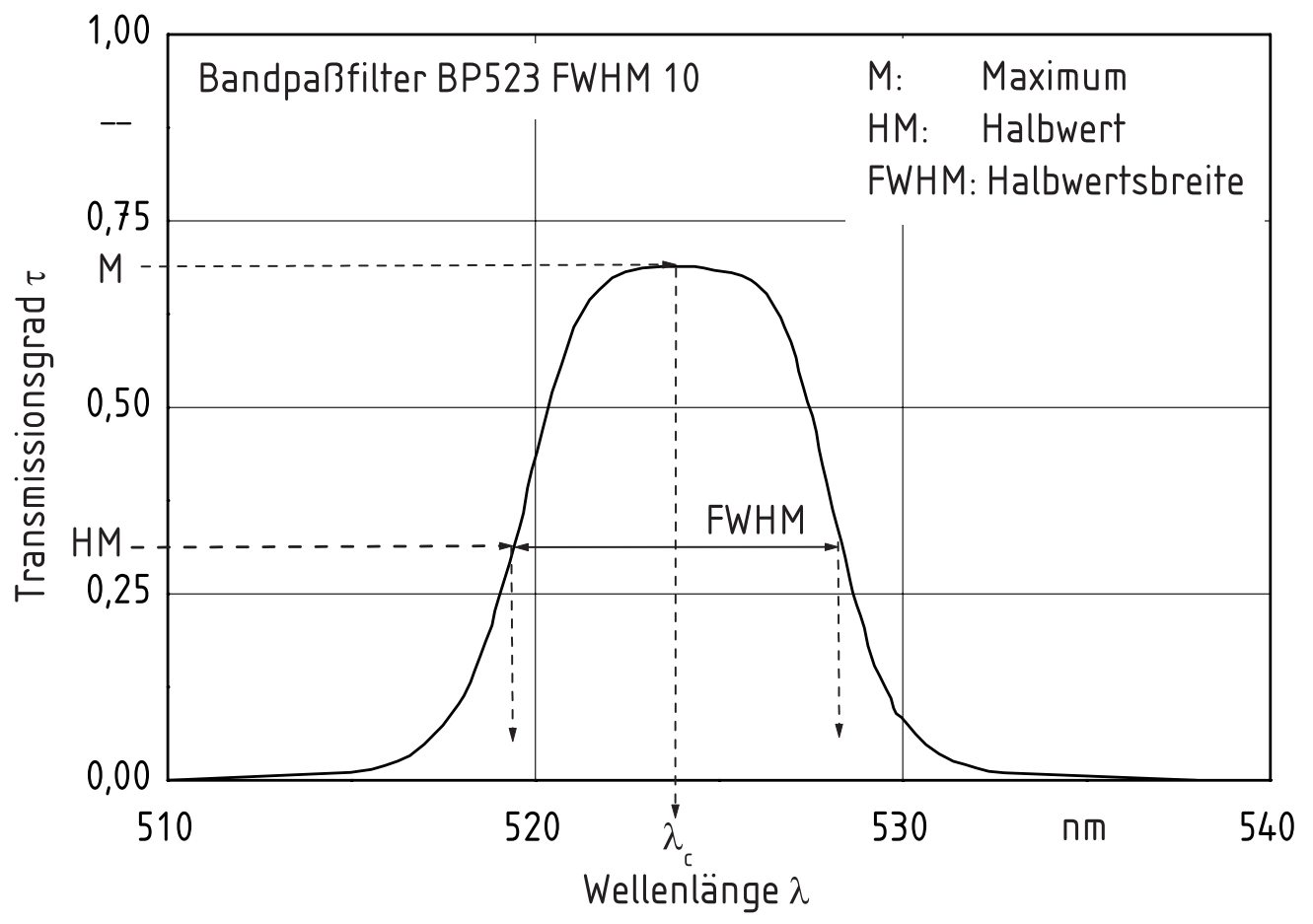

Bild 6.7: Transmissionsgrad in Abhängigkeit der Wellenlänge für den Filter BP523 FWHM 10 


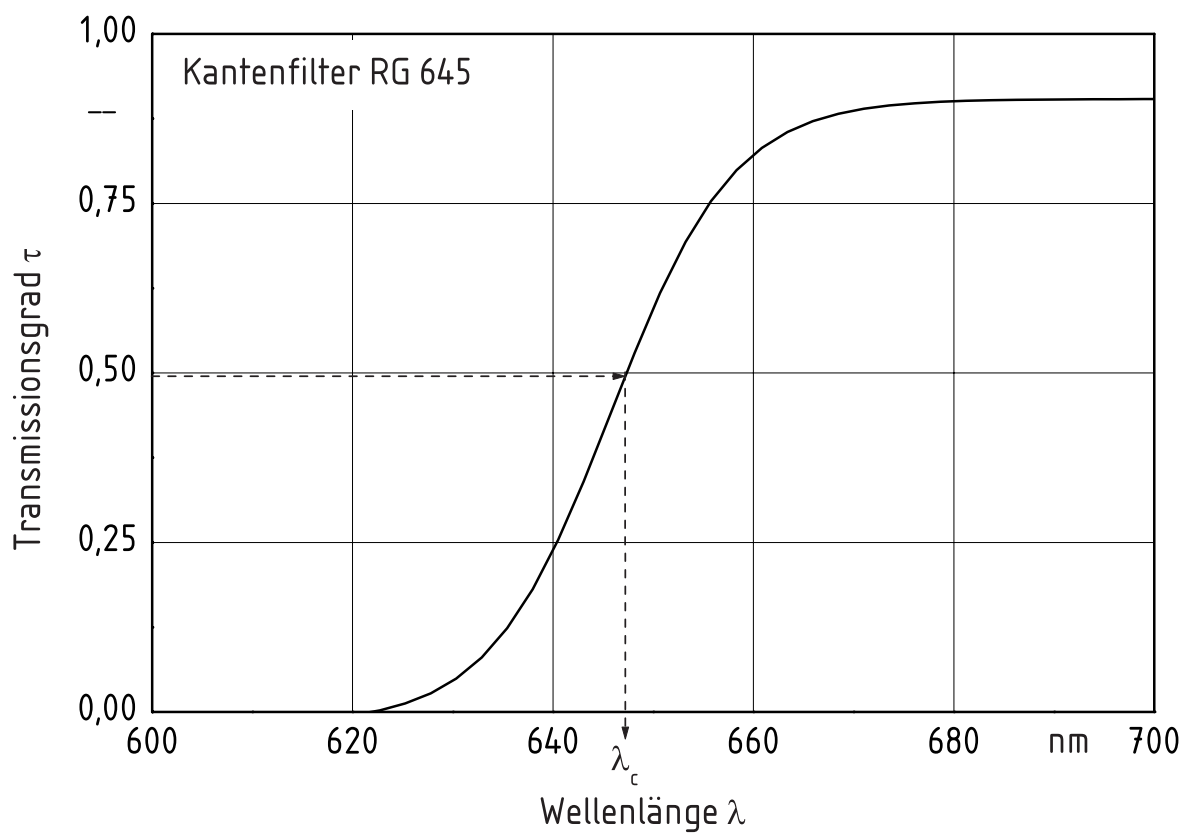

Bild 6.8: Transmissionsgrad in Abhängigkeit der Wellenlänge für den Filter RG645

muss. Wie in Kapitel 8.2.4 gezeigt wird, ist der Messfehler jedoch vernachlässigbar gering und eine Korrektur damit überflüssig.

In Bild 6.9 ist die relative Fluoreszenzintensität von fluo-4 in Abhängigkeit von der Emissionswellenlänge für unterschiedliche Konzentrationen an Calciumionen in der Lösung dargestellt. Der Maximalwert der Fluoreszenz ist konstant bei $\lambda=516 \mathrm{~nm}$ detektierbar, durch die Reaktion wird also keine Wellenlängenverschiebung ausgelöst. Liegt eine Calcium-freie Lösung vor, so ist eine geringe Hintergrundfluoreszenz $I_{F 1}^{\prime}$ detektierbar. Mit zunehmender Konzentration an freien Calcium-Ionen in der Lösung nimmt die Intensität der Fluoreszenz bis zu einem Maximalwert $I_{F 1}^{\prime \prime}$ für $c_{s a t}\left(C^{2+}\right)=39,6 \cdot 10^{-6} \mathrm{~mol} / \mathrm{l}[116]$ zu. Für die Mischversuche im Rührbehälter ist nur dieser Sättigungszustand entscheidend, da nicht die Konzentration der freien Calciumionen in der Lösung gemessen werden soll, sondern die Reaktion nur als Indikator für die erfolgte Mikromischung dient.

Für eine konstante Konzentration des Farbstoffs $c_{1, k}$ berechnet sich der Verstärkungsfaktor

$$
\beta=\frac{I_{F 1}^{\prime \prime}\left(c_{1, k}\right)}{I_{F 1}^{\prime}\left(c_{1, k}\right)}
$$

aus dem Verhältnis des Maximalwerts der Fluoreszenzintensität $I_{F 1}^{\prime \prime}$ zur Hintergrundfluoreszenz $I_{F 1}^{\prime}$. Für kleine Konzentrationen an Calciumionen ist zunächst ein starker Anstieg der Fluoreszenzemission zu verzeichnen, während der Maximalwert für die Sättigungskonzentration $c_{\text {sat }}$ nur langsam erreicht wird. Verunreinigungen mit Calcium müssen deshalb strikt ausgeschlossen werden, da sonst bereits ohne Zugabe von Calcium eine starke Hintergrundfluoreszenz messbar ist. Ziel ist es, einen möglichst großen dynamischen Bereich 


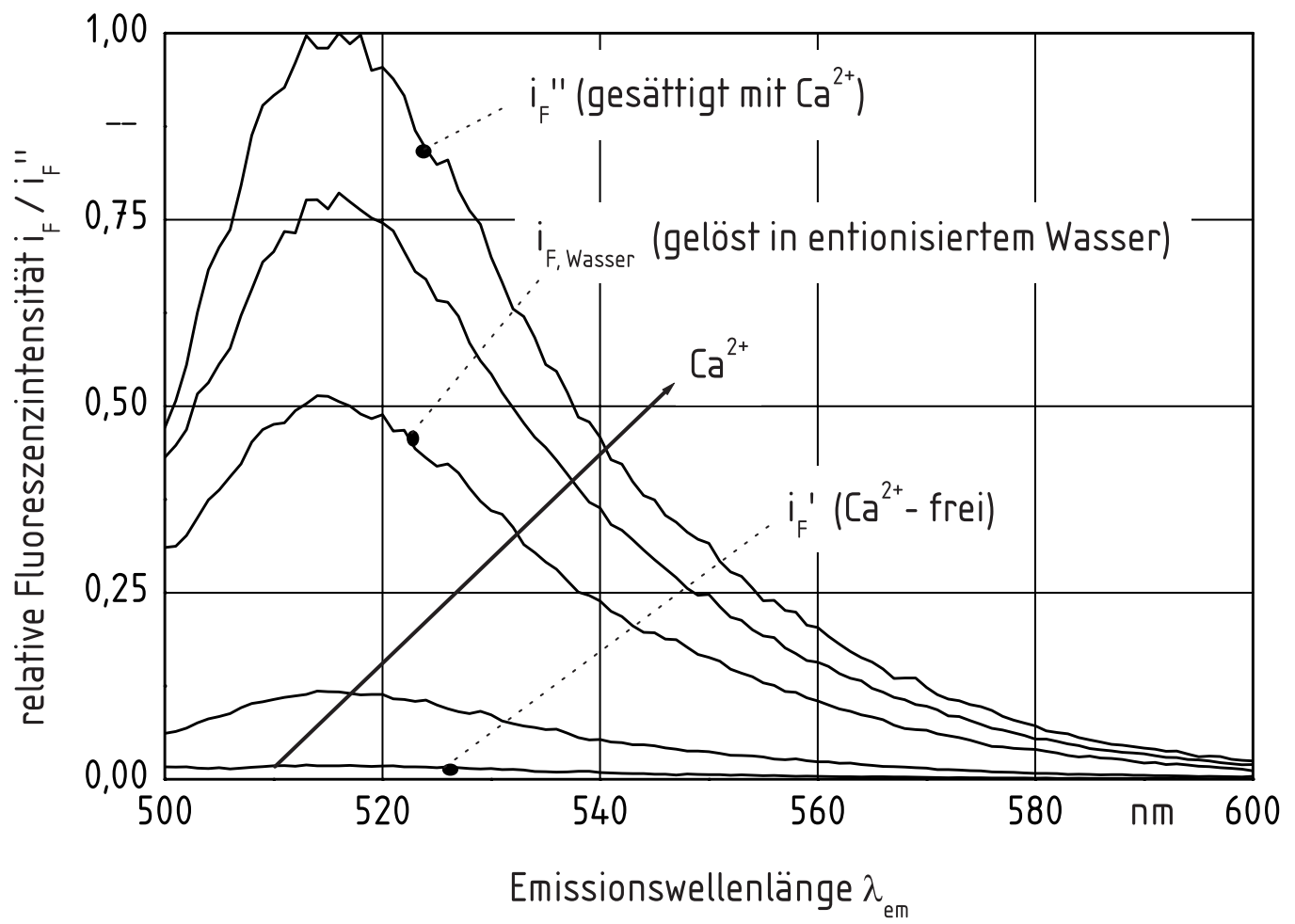

Bild 6.9: Relative Fluoreszenzintensität von fluo-4 in Abhängigkeit der Emissionswellenlänge für unterschiedliche Konzentrationen von freien Calciumionen in der Lösung

zwischen der Fluoreszenzemission im nicht-reagierten und der im abreagierten Zustand des Farbstoffs fluo-4, also einen großen Verstärkungsfaktor $\beta$, zu erzielen. Auf diese Weise kann ein gutes Signal-Rausch-Verhältnis bei der Detektion der Fluoreszenzemission erreicht werden. Die durch Verunreinigungen bedingte erhöhte Hintergrundfluoreszenz $I_{F 1}^{\prime}$ sollte deshalb minimal sein.

Für reinste Bedingungen sind Verstärkungsfaktoren von 40 bis zu 100 gemessen worden [116]. Es ist allerdings nahezu unmöglich, in einem nicht-sterilen verfahrenstechnischen Labor Verunreinigungen, die zu messbaren Fluoreszenzemissionen führen, gänzlich zu vermeiden. Eine mögliche Quelle für Verunreinigungen sind die zur Lagerung der Farbstoffösungen verwendeten Glas- oder Kunststoffbehältnisse, aus denen Calciumionen ausgelöst werden können. Außerdem enthalten einige Chemikalien, die z.B. zum Puffern des pH-Werts verwendet werden, herstellungsbedingt Verunreinigungen mit Calcium, die bereits zu messbaren Fluoreszenzemissionen von fluo-4 führen. Verunreinigungen mit Calcium können aber auch über das als Lösungsmittel verwendete Wasser eingetragen werden. Am Institut für Verfahrenstechnik steht eine Umkehrosmoseanlage zur Verfügung, die

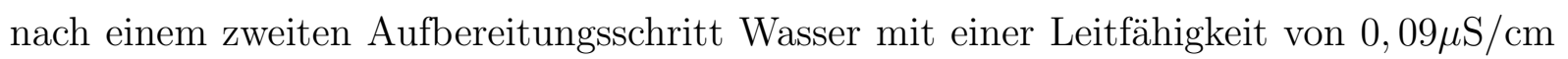
liefert, so dass Verunreinigungen des Wassers minimal sein dürften. Trotzdem ist bei Verwendung dieses Wassers als Lösungsmittel für fluo-4 eine bereits sehr stark erhöhte 
Fluoreszenzintensität messbar. In Bild 6.9 ist für eine konstante Konzentration an fluo-4 der Verlauf der Fluoreszenzintensität über der Wellenlänge für unterschiedliche Konzentrationen an freien Calciumionen in der Lösung aufgetragen. Für die Messungen wurde eine feste Anregungswellenlänge von $\lambda=494 \mathrm{~nm}$ gewählt. Besonders hervorzuheben sind die Kurven für die Calcium-freie Lösung $\left(i_{F 1}^{\prime}\right)$, für die mit Calciumionen gesättigte Lösung $\left(i_{F 1}^{\prime \prime}\right)$ sowie die für reines entionisiertes Wasser als Lösungsmittel $\left(i_{F 1, \text { Wasser }}\right)$. Es wird deutlich, dass die Hintergrundfluoreszenz bei der Verwendung von reinem Wasser bereits so groß ist, das nur noch ein Verstärkungsfaktor von 2-3 gemessen werden kann.

Daher wird eine Chemikalie, die eine höhere Affinität zu Calcium hat als fluo-4, der Farbstofflösung zugesetzt. Eine für diesen Zweck geeignete Chemikalie ist Ethylenglycolbis( $\beta$-aminoethylether)-N,N,N',N'-tetraessigsäure, kurz EGTA (Firma Sigma). EGTA bildet mit Calciumionen einen stabilen Koordinationskomplex, so dass die so gebundenen Calciumionen nicht mehr für die Reaktion mit fluo-4 zur Verfügung stehen und die Fluoreszenzemission sinkt. Bei einer Konzentration von $c_{E G T A}=7 \cdot 10^{-6} \mathrm{~mol} / \mathrm{l}$ kann $i_{F 1}^{\prime}$ aus Bild 6.9 gemessen werden. Durch Hinzufügen von Calciumionen (in Form von $\mathrm{CaCl}_{2}$-Lösung) steigt die Fluoreszenzintensität, bis sie bei der Konzentration von $c_{\mathrm{CaCl}_{2}}=6 \cdot 10^{-4} \mathrm{~mol} / \mathrm{l} \mathrm{ihr}$ Maximum $\left(i_{F 1}^{\prime \prime}\right)$ erreicht hat. Alle Calciumionen oberhalb der Sättigungskonzentration werden also an EGTA gebunden. Nur die Farbstofflösung wird deshalb mit EGTA der Konzentration $c_{E G T A}=7 \cdot 10^{-6} \mathrm{~mol} / \mathrm{l}$ versetzt. Im Rührbehälter wird hingegen die Konzentration $c_{\mathrm{CaCl}_{2}}=6 \cdot 10^{-4} \mathrm{~mol} / \mathrm{l}$ aber kein EGTA vorgelegt. Auf diese Weise ist sichergestellt, dass jedem Farbstoffmolekül des als Tropfen eingebrachten Farbstoffs eine ausreichende Menge an Calciumionen für die Reaktion zur Verfügung steht.

Der zweite Farbstoff, carboxy-SNARF, reagiert nicht mit Calcium oder EGTA und dient demnach als inerter Farbstoff. Allerdings ist die Fluoreszenzemission von carboxySNARF stark vom pH-Wert der Lösung abhängig. In Bild 6.10 sind die Absorptions- und Emissionsspektren von carboxy-SNARF für die pH-Werte 6 und 9 dargestellt. Mit steigenden pH-Werten ist eine deutliche Rotverschiebung sowohl beim Absorptions- als auch beim Emissionsspektrum zu erkennen. Da die Fluoreszenzemission des mit Calciumionen reagierenden Farbstoffs fluo-4 nicht vom pH-Wert abhängig ist, ist sowohl der pH-Wert als auch die Anregungswellenlänge so zu wählen, dass beide Farbstoffe gleichzeitig anregbar sind. Ein pH-Wert von 8,2 und eine Anregungswellenlänge von $\lambda_{e x}=495 \mathrm{~nm}$ haben sich als geeignet erwiesen. Der pH-Wert wird mit Hilfe eines Puffers eingestellt. Dieser muss sich wiederum inert zu dem reagierenden Farbstoff fluo-4 bzw. zu dessen Reaktionspartner Calcium verhalten. Ein geeigneter pH-Puffer ist Tris-(hydroxymethyl)-aminomethan, kurz Tris-Puffer (Firma Merck). Er liegt in Reinform kristallin vor. Durch Zugabe von entionisiertem Wasser und unterschiedlichen Mengen an Salzsäure $(\mathrm{HCl})$ kann eine Pufferlösung 


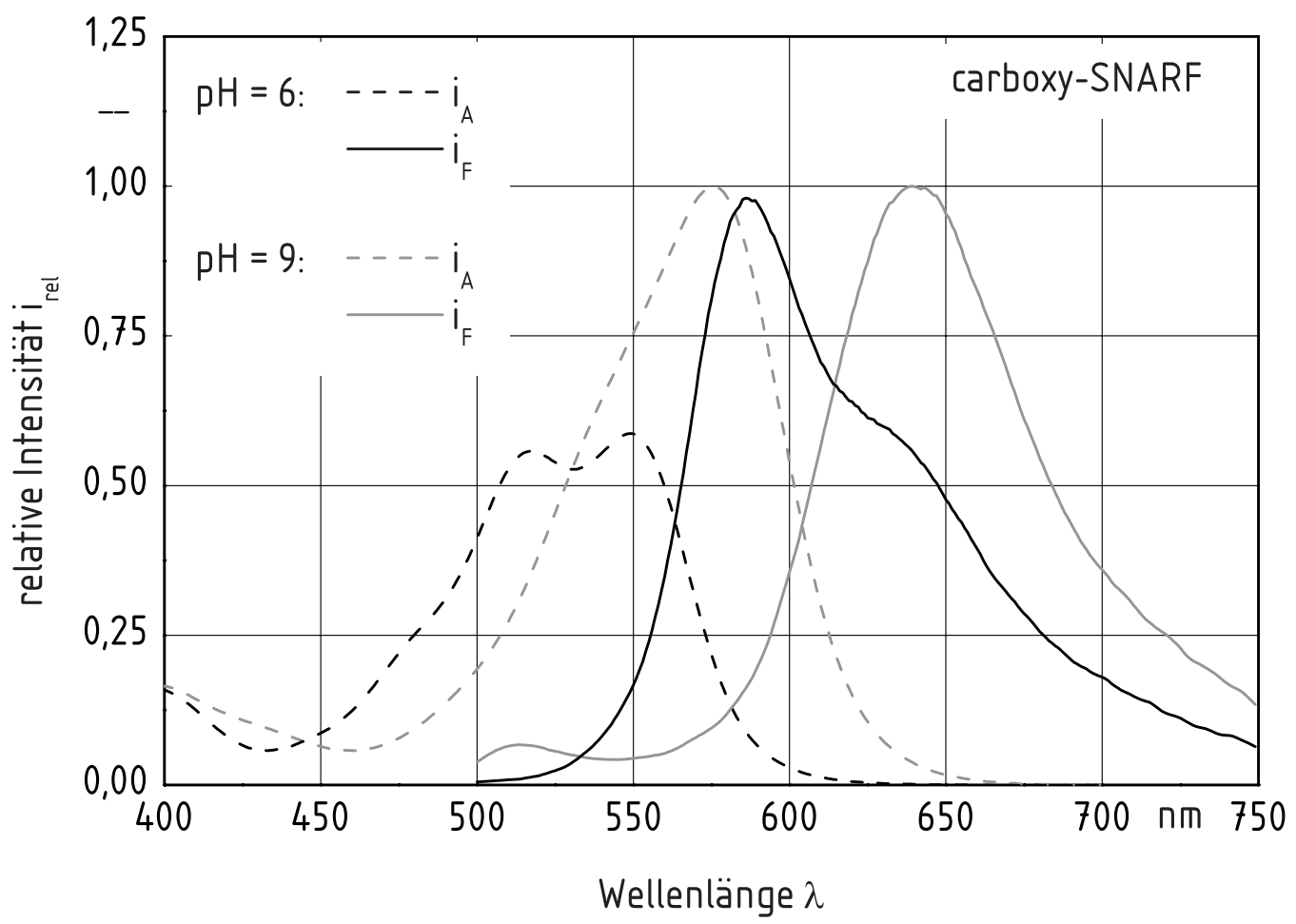

Bild 6.10: Absorptions- und Emissionsspektrum von carboxy-SNARF für $\mathrm{pH}=6$ und $\mathrm{pH}=9$

mit einem pH-Wert im Bereich von 7,1 bis 8,9 hergestellt werden. Die Konzentration der Pufferlösung beträgt 0,05 mol/l. Sowohl die Lösung im Rührbehälter als auch die Farbstofflösung, die in den Rührbehälter dosiert wird, sind mit der Pufferlösung vermischt.

Die Versuche werden mit einer wässrigen Lösung mit unterschiedlichen Massenanteilen an Carboxymethylzellulose (Typ: CRT 10000, Fa. Wolff Walsrode) durchgeführt. Die Zellulose erhöht die Viskosität der Flüssigkeit, so dass sich ein laminares Geschwindigkeitsprofil ausbildet. Außerdem erzeugt die Zellulose ein leicht strukturviskoses Fließverhalten. Die rheologischen Eigenschaften der wässrigen Zelluloselösungen sowie die resultierenden Strömungsfelder im Rührbehälter werden in Kapitel 7 näher erläutert.

\subsection{Versuchsdurchführung}

Die wässrige Zelluloselösung, die in das Rührgefäß dosiert wird, wird mit hochkonzentrierter Tris-Puffer-Lösung mit einem $\mathrm{pH}$-Wert von $\mathrm{pH}=8,2$ und EGTA-Lösung sowie mit den beiden Farbstoffen fluo-4 und carboxy-SNARF versetzt. Die Konzentrationen der Farbstoffe werden so gewählt, dass die Fluoreszenzintensität von fluo-4 im abreagierten Zustand etwa der von carboxy-SNARF entspricht. Dies ist für ein Anfangskonzentrationsverhältnis von $C_{0}=\frac{c_{2,0}}{c_{1,0}} \approx 2,15$ der Fall. Die Eingabe in das Rührgefäß erfolgt über eine Glaskapillare, die über einen Schlauch mit einer Dosiervorrichtung verbunden ist. 
Die Vorrichtung besteht aus einer Spritze, deren Kolben von einer Gewindestange mit Schrittmotorantrieb verfahren wird. Diese Anordnung ermöglicht eine genau definierte Zugabemenge und -geschwindigkeit. Falls nicht anders angegeben, wird $1 \mathrm{ml}$ des Farbstoffgemisches mit einem Volumenstrom von $0,5 \mathrm{ml} / \mathrm{s}$ in den Behälter dosiert. Vor der Farbstoffzufuhr wird ein stationäres Strömungsfeld im Rührbehälter eingestellt.

Die im Rührgefäß vorgelegte wässrige Zelluloselösung wird mit hochkonzentrierter TrisPuffer- Lösung mit einem $\mathrm{pH}$-Wert von $\mathrm{pH}=8,2$ und $\mathrm{CaCl}_{2^{-}}$Lösung versetzt. Die Konzentrationen aller verwendeten Stoffe sind in Tabelle 6.1 zusammengefasst. Die Konzentration von Calciumionen in der Lösung ist so bemessen, dass sich, trotz Zugabe von EGTA mit der Farbstofflösung, in der nächsten Umgebung des Tropfens genug Calciumionen für die Reaktion befinden. Auf diese Weise ist sichergestellt, dass die Komplexreaktion zwischen Calciumionen und fluo-4 nicht durch die Makromischung begrenzt wird.

Tabelle 6.1: Konzentrationen der Fluoreszenzfarbstoffe und Lösungsmittel im Rührbehälter und in der zudosierten Farbstofflösung)

\begin{tabular}{|l|c|c|}
\hline & Rührbehälter & Farbstoffösung \\
\hline Zellulose & $0,5 \% ; 1,0 \% ; 2,0 \%$ & $0,5 \% ; 1,0 \% ; 2,0 \%$ \\
Tris-Puffer & $0,05 \mathrm{~mol} / \mathrm{l}$ & $0,05 \mathrm{~mol} / \mathrm{l}$ \\
EGTA & - & $7 \cdot 10^{-6} \mathrm{~mol} / \mathrm{l}$ \\
$\mathrm{CaCl}_{2}$ & $6 \cdot 10^{-4} \mathrm{~mol} / \mathrm{l}$ & - \\
fluo-4 & - & $\approx 1 \cdot 10^{-6} \mathrm{~mol} / \mathrm{l}$ \\
carboxy-SNARF & - & $\approx 2 \cdot 10^{-6} \mathrm{~mol} / \mathrm{l}$ \\
\hline
\end{tabular}




\section{Strömungsfelder im Rührgefäß}

Im ersten Teil dieses Kapitels werden die geometrischen Abmessungen des verwendeten Rührbehälters und des Rührers angegeben. Anschließend werden Grundlagen und Ergebnisse der numerischen Berechnung des Strömungsfeldes im Rührbehälter erläutert.

\subsection{Rührgefäß und Rührer}

Die geometrischen Abmessungen des Rührers sowie der Einbau in das Rührgefäß ist in Bild 7.1 dargestellt. Der Rührbehälter hat den Außendurchmesser $D=100 \mathrm{~mm}$ und ist aus Duranglas gefertigt. Er besteht aus einem zylindrischen Rohr mit der Wandstärke 2,5 mm und einer Glasscheibe, die als Flachboden an das Rohr geklebt ist. Als Rührorgan wird ein Sechsblatt-Scheibenrührer verwendet. Er hat den Durchmesser $d_{r}=50 \mathrm{~mm}$ und ist mit dem Bodenabstand $h=50 \mathrm{~mm}$ oder $h=60 \mathrm{~mm}$ in den Behälter eingebaut. Die Rührerwelle und -scheibe sind aus Stahl gefertigt und zur Verminderung von Reflexionen geschwärzt. Die Rührerblätter bestehen aus einer durchsichtigen Teflonfolie mit der Dicke $1 \mathrm{~mm}$.

Das Volumen im Rührbehälter wird beim Erstellen des Rechengitters für die Simulation des Strömungsfeldes in Kapitel 7.4 möglichst genau nachgebildet.

\subsection{Transportgleichungen}

Die Strömung im Rührbehälter kann als isotherm, inkompressible, stationär und laminar betrachtet werden. Das Strömungsfeld lässt sich aus den Erhaltungsgleichungen für Masse und Impuls sowie geeigneten Materialmodellen für das Fließverhalten der gerührten Flüssigkeit errechnen. Für die mathematisch geschlossene Darstellung sind außerdem geeignete Randbedingungen zu formulieren.

Die Transportgleichungen für die Massen- und Impulsströme werden ausführlich von Bird, Steward und Lightfoot [117] hergeleitet und erläutert. Die lokale Massenbilanz für einen ortsfesten Raumpunkt lautet

$$
\frac{\partial \rho}{\partial t}+\operatorname{div}(\rho \vec{v})=0
$$

wobei $\rho$ die Dichte des Fluids, $t$ die Zeit und $\vec{v}$ der Geschwindigkeitsvektor ist. Für ein inkompressibles Fluid ist die Dichte konstant, und damit verschwindet die Divergenz des 

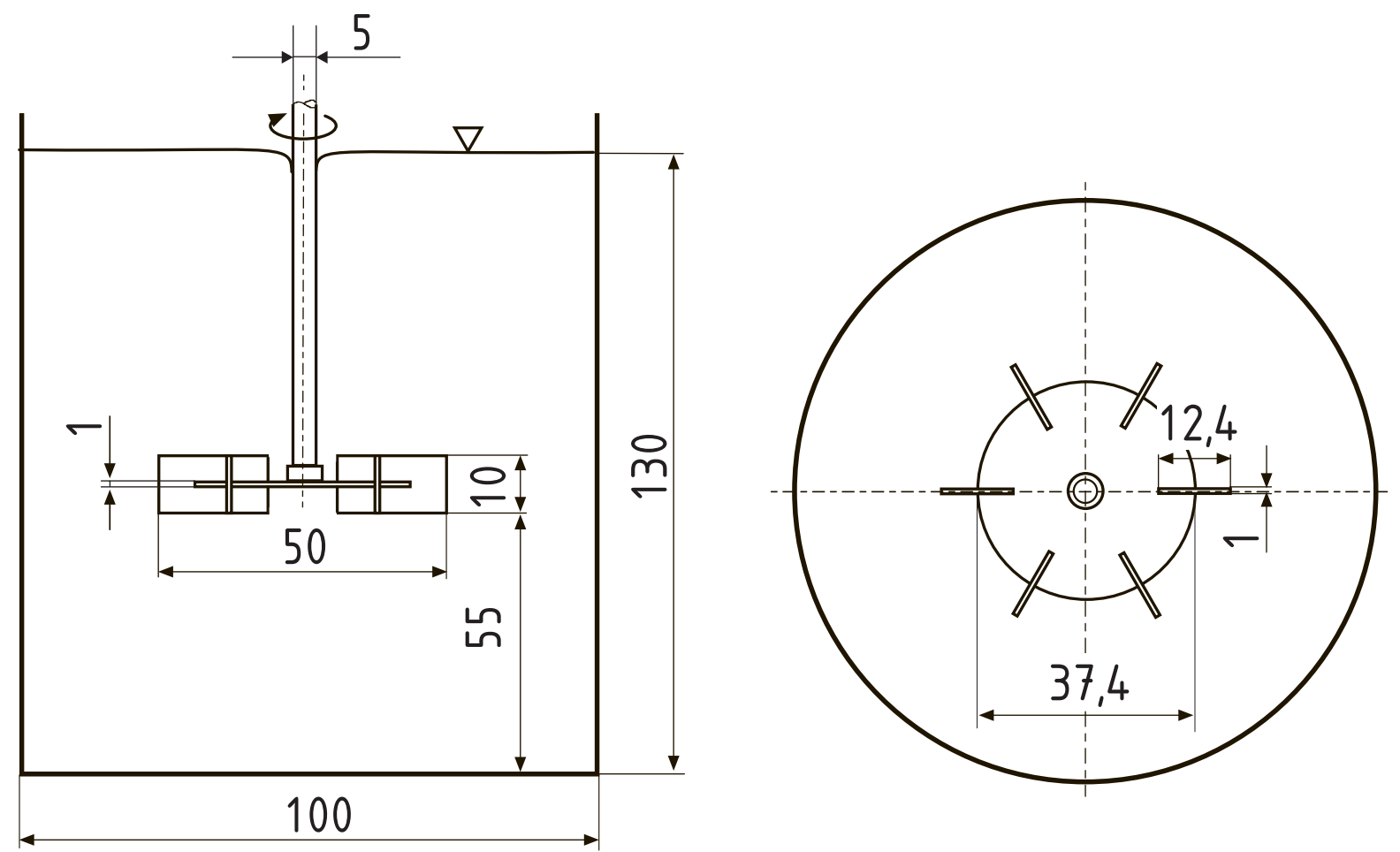

Bild 7.1: Geometrische Abmessungen von Rührbehälter und Rührer

Vektorfeldes

$$
\operatorname{div}(\vec{v})=0
$$

In kartesischen Koordinaten lautet die Beziehung

$$
\operatorname{div}(\vec{v})=\frac{\partial v_{x}}{\partial x}+\frac{\partial v_{y}}{\partial y}+\frac{\partial v_{z}}{\partial z}=0
$$

wobei $v_{i}$ die Komponenten des Geschwindigkeitsvektors $\vec{v}$ sind. Aufgrund der Achsensymmetrie des Behälters bietet sich die Berechnung der Transportgleichungen in zylindrischen Koordinaten an:

$$
\operatorname{div}(\vec{v})=\frac{\partial v_{x}}{\partial x}+\frac{\partial v_{r}}{\partial r}+\frac{v_{r}}{r}+\frac{1}{r} \frac{\partial v_{\theta}}{\partial \theta}=0
$$

wobei $v_{x}$ die Komponente des Geschwindigkeitsvektors in axialer, $v_{r}$ in radialer und $v_{\theta}$ in Umfangsrichtung darstellt. Der Term $\frac{v_{r}}{r}$ ergibt sich durch die Koordinatentransformation und folgt aus der Änderung eines Kontrollvolumens $\mathrm{d} V=r \mathrm{~d} \theta \cdot \mathrm{d} x \cdot \mathrm{d} r$ mit dem Radius $r$.

Die Bilanz der Impulsstromdichte $\rho \vec{v}$ liefert die Bewegungsgleichung. Sie berücksichtigt, dass ein in der Strömung mitbewegtes, infinitesimales Volumenelement aufgrund von äußeren Kräften beschleunigt wird. Für einen ortsfesten Raumpunkt ergibt sich in vektorieller Darstellung

$$
\rho \frac{D \vec{v}}{D t}=-\operatorname{grad}(p)-\operatorname{div}(\underline{\underline{\tau}})+\rho \vec{g}
$$

Hierin ist

$$
\frac{D \vec{v}}{D t}=\frac{\partial \vec{v}}{\partial t}+\vec{v} \cdot \operatorname{grad}(\vec{v})
$$


die substantielle Zeitableitung des Geschwindigkeitsvektors $\vec{v}, p$ der hydrostatische Druck, $\vec{g}$ die Erdbeschleunigung und $\underline{\underline{\tau}}$ der Spannungstensor mit den Komponenten in Zylinderkoordinaten

$$
\underline{\underline{\tau}}=\left(\begin{array}{ccc}
\tau_{x x} & \tau_{x r} & \tau_{x \theta} \\
\tau_{r x} & \tau_{r r} & \tau_{r \theta} \\
\tau_{\theta x} & \tau_{\theta r} & \tau_{\theta \theta}
\end{array}\right) .
$$

Der erste Index $i$ der Spannungskomponenten $\tau_{i j}$ bezeichnet jeweils die Koordinatenrichtung senkrecht zur Schnittfläche des Volumenelements. Der zweite Index $j$ gibt die Wirkrichtung der Komponente des auf diese Schnittfläche einwirkenden Spannungsvektors an. Für $i=j$ handelt es sich demnach um Normalspannungen, andernfalls um Schubspannungen. Aus der Momentenbilanz folgt die Symmetrie des Spannungstensors

$$
\tau_{i j}=\tau_{j i}
$$

so dass sich die Anzahl der unbekannten Komponenten des Spannungstensors von 9 auf 6 reduziert.

Für eine stationäre Strömung lautet die Impulsbilanz in komponentenweiser Darstellung für die drei Strömungsrichtungen in zylindrischen Koordinaten

$$
\begin{aligned}
\rho\left(v_{x} \frac{\partial v_{x}}{\partial x}+v_{r} \frac{\partial v_{x}}{\partial r}+\frac{v_{\theta}}{r} \frac{\partial v_{x}}{\partial \theta}\right)= & -\frac{\partial p}{\partial x}-\left(\frac{\partial \tau_{x x}}{\partial x}+\frac{1}{r} \frac{\partial}{\partial r}\left(r \tau_{r x}\right)+\frac{1}{r} \frac{\partial \tau_{\theta x}}{\partial \theta}\right) \\
& +\rho g_{x}, \\
\rho\left(v_{x} \frac{\partial v_{r}}{\partial x}+v_{r} \frac{\partial v_{r}}{\partial r}+\frac{v_{\theta}}{r} \frac{\partial v_{r}}{\partial \theta}-\frac{v_{\theta}^{2}}{r}\right)= & -\frac{\partial p}{\partial r}-\left(\frac{\partial \tau_{r x}}{\partial x}+\frac{1}{r} \frac{\partial}{\partial r}\left(r \tau_{r r}\right)+\frac{1}{r} \frac{\partial \tau_{r \theta}}{\partial \theta}-\frac{\tau_{\theta \theta}}{r}\right) \\
& +\rho g_{r}, \\
\rho\left(v_{x} \frac{\partial v_{\theta}}{\partial x}+v_{r} \frac{\partial v_{\theta}}{\partial r}+\frac{v_{\theta}}{r} \frac{\partial v_{\theta}}{\partial \theta}+\frac{v_{r} v_{\theta}}{r}\right)= & -\frac{1}{r} \frac{\partial p}{\partial \theta}-\left(\frac{\partial \tau_{\theta x}}{\partial x}+\frac{1}{r^{2}} \frac{\partial}{\partial r}\left(r^{2} \tau_{\theta r}\right)+\frac{1}{r} \frac{\partial \tau_{\theta \theta}}{\partial \theta}\right) \\
& +\rho g_{\theta} .
\end{aligned}
$$

In Gl. (7.10) ist $\rho \frac{v_{\theta}^{2}}{r}$ die Zentrifugalkraft in radialer Richtung, die durch die Rotationsbewegung des Fluids in Umfangsrichtung $\theta$ hervorgerufen wird. Dieser Term entsteht automatisch bei der Transformation von kartesischen zu Zylinderkoordinaten. Gleiches gilt für die Corioliskraft $\rho \frac{v_{r} v_{\theta}}{r}$ in Gl.(7.11), die in der Umfangsrichtung wirkt und durch eine gleichzeitige Bewegung in radialer und Umfangsrichtung hervorgerufen wird. In einem senkrecht stehenden Rührbehälter gilt außerdem $g_{x}=g, g_{r}=g_{\theta}=0$.

\subsection{Rheologische Fließgesetze}

Zur Berechnung des Strömungsfeldes muss der Zusammenhang zwischen Spannungs- und Deformationszustand bekannt sein. Dieser Zusammenhang wird mit dem rheologischen Fließgesetz beschrieben, welches für die zu mischenden Fluide zu formulieren ist. 


\subsubsection{Newtonsche Fluide}

In einem strömenden Fluid werden die einzelnen Fluidelemente nicht nur in ihrer Lage verschoben, sondern unter dem Einfluss der Normalspannungen $\tau_{i i}$ und der Schubspannungen $\tau_{i j}$ auch verformt. Die Verformungsgeschwindigkeit hängt von der Relativbewegung der einzelnen Massenpunkte zueinander ab. Diese wird an jedem Ort durch den Geschwindigkeitsgradiententensor

$$
\underline{\underline{L}}=\operatorname{grad}(\vec{v})=\left(\begin{array}{ccc}
\frac{\partial v_{x}}{\partial x} & \frac{\partial v_{x}}{\partial y} & \frac{\partial v_{x}}{\partial z} \\
\frac{\partial v_{y}}{\partial x} & \frac{\partial v_{y}}{\partial y} & \frac{\partial v_{y}}{\partial z} \\
\frac{\partial v_{z}}{\partial x} & \frac{\partial v_{z}}{\partial y} & \frac{\partial v_{z}}{\partial z}
\end{array}\right)
$$

beschrieben [118]. Dieser kann entsprechend

$$
\underline{\underline{L}}=\frac{1}{2}\left(\underline{\underline{L}}+\underline{\underline{L}}^{T}\right)+\frac{1}{2}\left(\underline{\underline{L}}-\underline{\underline{L}}^{T}\right)=\underline{\underline{D}}+\underline{\underline{W}}
$$

in den symmetrischen Deformationsgeschwindigkeitstensor

$$
\underline{\underline{D}}=\frac{1}{2}\left(\begin{array}{ccc}
2 \frac{\partial v_{x}}{\partial x} & \left(\frac{\partial v_{x}}{\partial y}+\frac{\partial v_{y}}{\partial x}\right) & \left(\frac{\partial v_{x}}{\partial z}+\frac{\partial v_{z}}{\partial x}\right) \\
\left(\frac{\partial v_{x}}{\partial y}+\frac{\partial v_{y}}{\partial x}\right) & 2 \frac{\partial v_{y}}{\partial y} & \left(\frac{\partial v_{y}}{\partial z}+\frac{\partial v_{z}}{\partial y}\right) \\
\left(\frac{\partial v_{x}}{\partial z}+\frac{\partial v_{z}}{\partial x}\right) & \left(\frac{\partial v_{y}}{\partial z}+\frac{\partial v_{z}}{\partial y}\right) & 2 \frac{\partial v_{z}}{\partial z}
\end{array}\right)
$$

und den schiefsymmetrischen Drehgeschwindigkeitstensor

$$
\underline{\underline{W}}=\frac{1}{2}\left(\begin{array}{ccc}
0 & \left(\frac{\partial v_{x}}{\partial y}+\frac{\partial v_{y}}{\partial x}\right) & \left(\frac{\partial v_{x}}{\partial z}+\frac{\partial v_{z}}{\partial x}\right) \\
\left(\frac{\partial v_{x}}{\partial y}+\frac{\partial v_{y}}{\partial x}\right) & 0 & \left(\frac{\partial v_{y}}{\partial z}+\frac{\partial v_{z}}{\partial y}\right) \\
\left(\frac{\partial v_{x}}{\partial z}+\frac{\partial v_{z}}{\partial x}\right) & \left(\frac{\partial v_{y}}{\partial z}+\frac{\partial v_{z}}{\partial y}\right) & 0
\end{array}\right)
$$

aufgeteilt werden. Dabei ist $\underline{\underline{L}}^{T}$ der transponierte Geschwindigkeitsgradiententensor, der durch Vertauschen von Zeilen und Spalten aus $\underline{\underline{L}}$ hervorgeht. Die Deformation der Fluidelemente wird durch den Deformationsgeschwindigkeitstensor $\underline{\underline{D}}$ beschrieben. Gilt an einer Stelle im Strömungsfeld $\underline{\underline{D}}=0$, so besteht die Relativbewegung der unmittelbaren Umgebung des Punktes lediglich aus einer Drehung. Wird ein Flächen- oder Volumenelement betrachtet, so behält dieses seine ursprüngliche Form und Größe bei, es führt eine reine Drehbewegung aus.

Für ein inkompressibles newtonsches Fluid wird die Annahme getroffen, dass der Spannungstensor $\underline{\underline{\tau}}$ in Gl. (7.5) dem Deformationsgeschwindigkeitstensor $\underline{\underline{D}}$ proportional ist. Das entsprechende rheologische Fließgesetz, welches den Spannungstensor in den Impulsbilanzgleichungen ersetzt, lautet dann

$$
\underline{\underline{\tau}}=\eta(2 \underline{\underline{D}}) .
$$


Im eindimensionalen Fall ergibt sich daraus der bekannte newtonsche Schubspannungsansatz

$$
\tau_{x y}=\eta \frac{\partial v_{x}}{\partial y}=\eta \dot{\gamma}
$$

Die Viskosität $\eta$ ist auf experimentellem Wege zu ermitteln und beschreibt als einziger Parameter das Fließverhalten.

\subsubsection{Nicht-newtonsche Fluide}

Für nicht-newtonsche Fluide ist die Viskosität $\eta$ hingegen eine Funktion der Deformationsgeschwindigkeiten und damit kein konstanter Parameter, der allein ausreichend wäre, das Fließverhalten zu beschreiben. Die Invarianten des Deformationsgeschwindigkeitstensors sind prinzipiell ein geeignetes Maß für die Deformation. Sie haben den Vorteil, dass sie unabhängig von einer Koordinatentransformation sind. Die erste Invariante

$$
I_{1}=\operatorname{tr}(\underline{\underline{D}})
$$

ist die Spur des Tensors $\underline{\underline{D}}$, die gleich der Summe seiner Diagonalelemente ist. Für inkompressible Fluide ist gemäß Gl.(7.2) die erste Invariante $I_{1}=0$, da die Spur von $\underline{\underline{D}}$ der Divergenz des Geschwindigkeitsfeldes entspricht. Für ebene Strömungen (z.B. $v_{z}=0$, $\left.\frac{\partial v_{x}}{\partial z}=0, \frac{\partial v_{y}}{\partial z}=0\right)$ verschwindet die dritte Invariante

$$
I_{3}=\operatorname{det}(\underline{\underline{D}})=0 \text {. }
$$

Aus diesem Grund wird in den meisten rheologischen Fließgesetzen die zweite Invariante $I_{2}$ des Deformationsgeschwindigkeitstensors

$$
I_{2}=\frac{1}{2}\left[(\operatorname{tr}(\underline{\underline{D}}))^{2}-\operatorname{tr}\left(\underline{\underline{D}}^{2}\right)\right]
$$

als Maß für die Deformation verwendet. Ein rheologisches Fließgesetz, welches das Fließverhalten nicht-newtonscher Fluide beschreibt, ist z.B. das Potenzgesetz von Ostwald-deWaele

$$
\underline{\underline{\tau}}=K\left|I_{2}(2 \underline{\underline{D}})\right|^{\frac{n-1}{2}}(2 \underline{\underline{D}}) \text {. }
$$

Darin bezeichnen $K$ den Konsistenzfaktor und $n$ den Fließexponenten. Beide Werte sind stoffspezifische Größen. Für $n>1$ steigt die Viskosität mit der Deformationsgeschwindigkeit an, d.h. es liegt dilatantes Fließverhalten vor. Für $n<1$ sinkt die Viskosität mit der Deformationsgeschwindigkeit, was als strukturviskos bezeichnet wird.

Für eine einfache Scherströmung lautet die zweite Invariante des Deformationsgeschwindigkeitstensors

$$
\left|I_{2}(2 \underline{\underline{D}})\right|=\left(\frac{\partial v_{x}}{\partial y}\right)^{2}=\dot{\gamma}^{2}
$$


Hiermit nimmt das Fließgesetz die bekannte Form

$$
\tau_{x y}=K \dot{\gamma}^{n} \quad \text { oder } \quad \eta(\dot{\gamma})=K \dot{\gamma}^{n-1}
$$

an.

Für strukturviskose Fluide ist das Modell nach Bird-Carreau [119]

$$
\frac{\eta(\dot{\gamma})-\eta_{\infty}}{\eta_{0}-\eta_{\infty}}=\left(1+(K \cdot \dot{\gamma})^{2}\right)^{\frac{n-1}{2}}
$$

insbesondere für kleine Deformationsgeschwindigkeiten besser geeignet. In Gl. (7.24) bezeichnen $\eta_{0}$ die Nullviskosität für kleine Deformationsgeschwindigkeiten, $\eta_{\infty}$ die Endviskosität für sehr große Deformationsgeschwindigkeiten, $K$ den Konsistenzfaktor und $n$ den Fließexponenten. Die Parameter müssen aus gemessenen Fließkurven berechnet werden.

\subsection{Rechengitter und Randbedingungen}

Aus den Transportgleichungen für die Massen- und Impulsströme ergibt sich ein System von nichtlinearen gekoppelten partiellen Differentialgleichungen. Dieses kann im allgemeinen nur numerisch vollständig gelöst werden. Das Strömungsfeld im Rührbehälter wird mit dem kommerziellen Simulationsprogramm CFX 4.4 nach der Methode der Finiten Volumen berechnet. Dazu wird der Rührbehälter in einzelne Kontrollvolumen diskretisiert, und die Transportgleichungen für Masse und Impuls werden für jedes Kontrollvolumen mit geeigneten Randbedingungen numerisch gelöst. Die örtliche Diskretisierung des konvektiven Transports erfolgt mit einem Verfahren 2. Ordnung, dem 'Higher Upwind' Verfahren. Dabei werden nicht nur die Werte in den benachbarten Zellen, zwischen denen der Transport berechnet werden soll, sondern auch der Wert in der übernächsten Zelle berücksichtigt.

Das Rechengitter und die Randbedingungen sind schematisch in Bild 7.2 dargestellt. Es handelt sich um ein blockstrukturiertes Gitter aus fünf Blöcken. Ein zentraler Block enthält die Rührerwelle und -scheibe sowie die sechs Rührerblätter. Sie werden als Festkörper mit Haftung als Randbedingung modelliert, wobei die Dicke von Scheibe und Rührerblättern vernachlässigt wird. In Rührernähe ist eine lokale Verfeinerung des Netzes durchgeführt worden. Die Drehung des Rührorgans wird mit Hilfe der Multiple Frame of Reference (MFR) Methode simuliert [120]. Dabei wird die Strömung im Bereich des Rührers in einem mit der Rührerdrehzahl mitrotierenden Koordinatensystem berechnet. Dies ist notwendig, um die Geometrie der rotierenden Rührerblätter in einer raumfesten Euler-Beschreibung abbilden zu können. Der rotierende Bereich umfasst den oben beschriebenen zentralen Block mit dem Rührorgan und ist so gewählt, dass die periodischen 


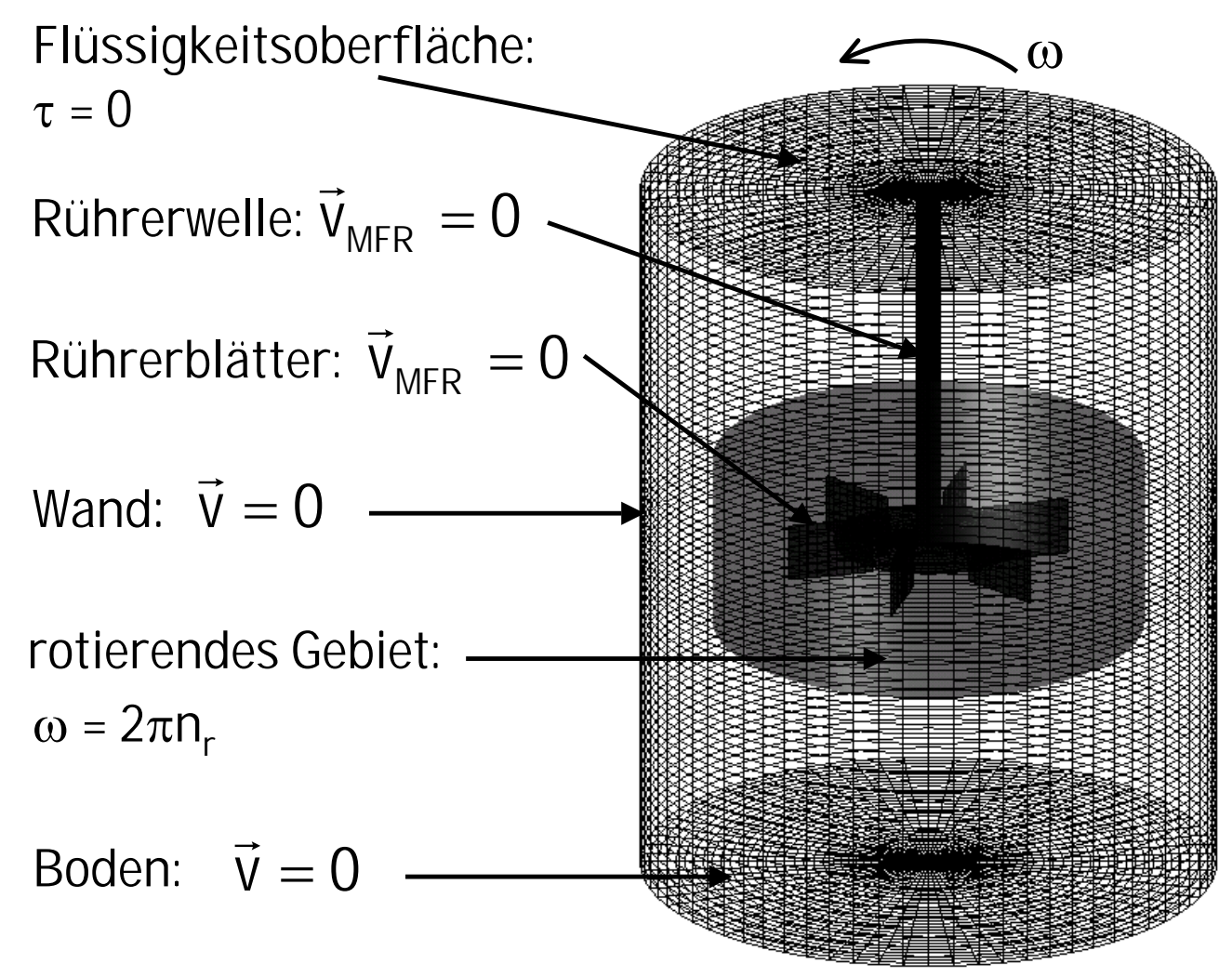

Bild 7.2: Schematische Darstellung des Rechengitters und der Randbedingungen

Schwankungen am Rand möglichst gering sind. Die zeitgemittelte Lösung des rotierenden Gebietes wird an den Rändern mit dem übrigen Strömungsfeld gekoppelt, welches als stationär betrachtet wird.

Alternativ ist die Modellierung der instationären Strömung im Rührerbereich mit Hilfe des Sliding Mesh-Verfahrens möglich. Auch hier wird der Bereich um den Rührer durch ein rotierendes Gitter aufgelöst und der restliche Bereich durch ein stationäres Gitter diskretisiert. Die Lösung im Rührerbereich wird aber nach jedem Zeitschritt mit dem übrigen Rechengebiet gekoppelt. Das heißt, die im rotierenden Gebiet erhaltenen Randwerte werden für das stationäre Berechnungsgebiet als Randbedingungen vorgegeben. Da für jeden Zeitschritt eine vollständige Lösung im gesamten Gebiet berechnet wird, ist dieses Verfahren sehr aufwändig. In dieser Arbeit ist nur das stationäre Strömungsfeld von Interesse ist, so dass die MFR-Methode ausreichend genau ist.

Für die Wände und den Boden des Rührbehälters wird als Randbedingung Haftung mit $\vec{v}=0$ angenommen. Wandhaftung muss auch an den Rührerblättern und an der Rührerwelle gelten. Im mitrotierenden Koordinatensystem gilt dann $\vec{v}_{M F R}=0$. Die Flüssigkeitsoberfläche ist schubspannungsfrei. Das strukturviskose Verhalten der Zelluloselösung wird mit dem Bird-Carreau Modell modelliert. Als repräsentative Deformationsgeschwindigkeit 


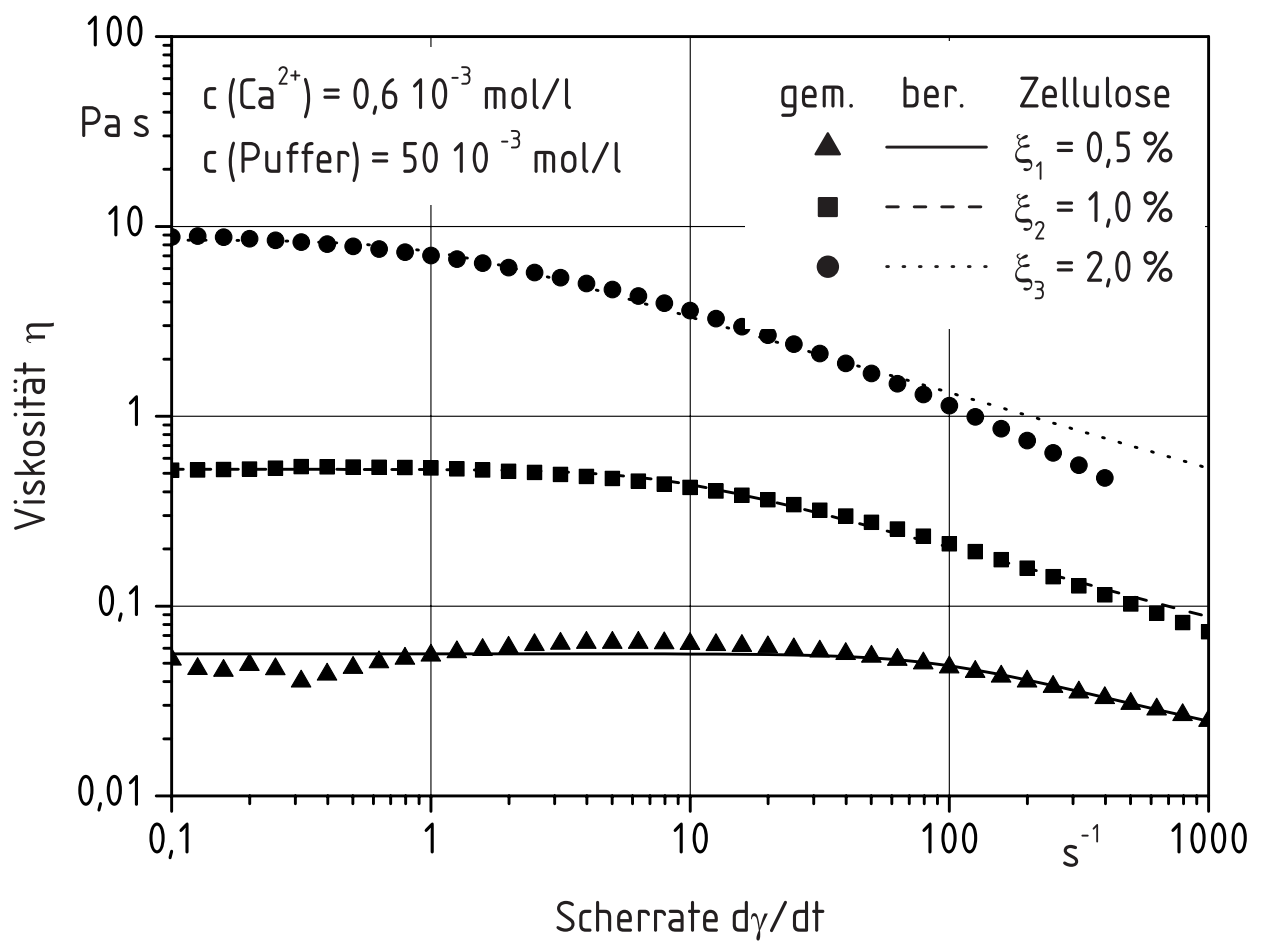

Bild 7.3: Viskosität in Abhängigkeit von der Scherrate für unterschiedlich konzentrierte Zelluloselösungen

$\dot{\gamma}$ wird die in Gl. (7.20) hergeleitete zweite Invariante des Deformationsgeschwindigkeitstensors $I_{2}$ verwendet. Für ein inkompressibles Fluid vereinfacht sich Gl. (7.20) zu

$$
\begin{aligned}
\dot{\gamma}= & {\left[2\left\{\left(\frac{\partial v_{x}}{\partial x}\right)^{2}+\left(\frac{\partial v_{y}}{\partial y}\right)^{2}+\left(\frac{\partial v_{z}}{\partial z}\right)^{2}\right\}+\right.} \\
& \left.\left(\frac{\partial v_{x}}{\partial y}+\frac{\partial v_{y}}{\partial x}\right)^{2}+\left(\frac{\partial v_{x}}{\partial z}+\frac{\partial v_{z}}{\partial x}\right)^{2}+\left(\frac{\partial v_{y}}{\partial z}+\frac{\partial v_{z}}{\partial y}\right)^{2}\right]^{\frac{1}{2}} .
\end{aligned}
$$

Die Parameter des Modells in Gl. (7.24) werden so gewählt, dass sie das Fließverhalten der in Kapitel 9 verwendeten wässrigen Zelluloselösungen beschreiben. Es werden drei Lösungen mit unterschiedlichen Massenanteilen an Zellulose verwendet. In Bild 7.3 sind die gemessenen Fließkurven für Zelluloselösungen mit einem Massenanteil der Zellulose von $\xi_{1}=0,5 \%, \xi_{2}=1,0 \%$ bzw. $\xi_{3}=2,0 \%$ dargestellt. Es ist der für strukturviskose Flüssigkeiten übliche degressive Verlauf zu erkennen. Die Nullviskosität steigt mit zunehmendem Massenanteil an Zellulose. Die ebenfalls eingezeichneten Ausgleichskurven wurden nach dem Modell von Bird-Carreau modelliert. Die zugehörigen Parameter gemäß Gl. (7.24) sind in Tabelle 7.1 zusammengefasst. 
Tabelle 7.1: Konzentrationen der Fluoreszenzfarbstoffe und Lösungsmittel im Rührbehälter und in der zudosierten Farbstofflösung

\begin{tabular}{|c|c|c|c|c|c|}
\hline $\mathrm{i}$ & $\begin{array}{c}\text { Massenanteil } \\
\text { Zellulose } \xi_{i} \\
\%\end{array}$ & $\begin{array}{c}\text { Nullviskosität } \\
\eta_{0} \\
{[\mathrm{~Pa} \mathrm{~s}]}\end{array}$ & $\begin{array}{c}\text { Endviskosität } \\
\eta_{\infty} \\
{[\mathrm{Pa} \mathrm{s}]}\end{array}$ & $\begin{array}{c}\text { Konsistenz- } \\
\text { faktor K } \\
{[-]}\end{array}$ & $\begin{array}{c}\text { Fließ- } \\
\text { exponent n } \\
{[-]}\end{array}$ \\
\hline 1 & 0,5 & 0,05603 & 0,00666 & 0,01098 & 0,58113 \\
2 & 1,0 & 0,52532 & $7,9419 \cdot 10^{-15}$ & 0,13193 & 0,63324 \\
3 & 2,0 & 8,5 & $2,0221 \cdot 10^{-8}$ & 1,0333 & 0,60041 \\
\hline
\end{tabular}

\subsection{Berechnete Geschwindigkeitsfelder}

Gemäß der in Kapitel 9.3 auf experimentellem Wege untersuchten Strömungsfelder werden die lokalen Geschwindigkeiten für die oben genannten wässrigen Zelluloselösungen berechnet. Ähnliche Strömungsfelder werden für eine konstante Reynoldszahl

$$
R e=\frac{n_{r} d_{r}^{2} \rho}{\eta}
$$

erzielt. Dabei sind $n_{r}$ die Drehzahl und $d_{r}$ der Durchmeser des Rührorgans, $\rho$ ist die Dichte und $\eta$ ist die Viskosität des Fluides. Da die Viskosität für strukturviskose Fluide nicht konstant ist, kann die Re-Zahl nicht direkt berechnet werden. Eine empirische Methode, mit der eine repräsentative Reynoldszahl berechnet werden kann, wird in Kapitel 9.3 vorgestellt. Daraus ergibt sich für eine Reynoldszahl von $R e=40$ eine Rührerdrehzahl von $n_{r 1}=60 \mathrm{~min}^{-1}$ für die Zelluloselösung mit $\xi_{1}=0,5 \%, n_{r 2}=250 \mathrm{~min}^{-1}$ für die Zelluloselösung mit $\xi_{2}=1,0 \%$ und $n_{r 3}=750 \mathrm{~min}^{-1}$ für die Zelluloselösung mit $\xi_{3}=2,0 \%$.

In Bild 7.4 ist das Geschwindigkeitsfeld für die 0,5\%ige Zelluloselösung projiziert auf die Ebene zwischen den Rührerblättern (x-z-Ebene) als Vektordarstellung gezeigt. Die für einen radial fördernden Rührer wie den Scheibenrührer typischen, torusförmigen Ringwirbel ober- und unterhalb der Rührerebene sind zu erkennen. Das Fluid wird durch die Verdrängung der Rührerscheiben zunächst in Umfangsrichtung beschleunigt. Die dadurch induzierte Strömung heißt Primärströmung. Sie bewirkt eine Zentrifugalbeschleunigung der Fluidelemente, so dass diese nach außen zur Behälterwand hin transportiert werden. Am Bifurkationspunkt wird die Flüssigkeit entweder nach oben oder nach unten transportiert. Aus Kontinuitätsgründen muss Flüssigkeit von oben und unten in die Rührerebene nachströmen, so dass zwei in sich geschlossene Ringwirbel entstehen. Diese induzierte Strömung heißt Sekundärströmung. Der Stoffaustausch über die Rührerebene hinweg ist für den stationären Strömungszustand im laminaren Bereich sehr gering.

Außerdem ist der Betrag der Geschwindigkeitsvektoren in Bild 7.4 farbig codiert. In der 


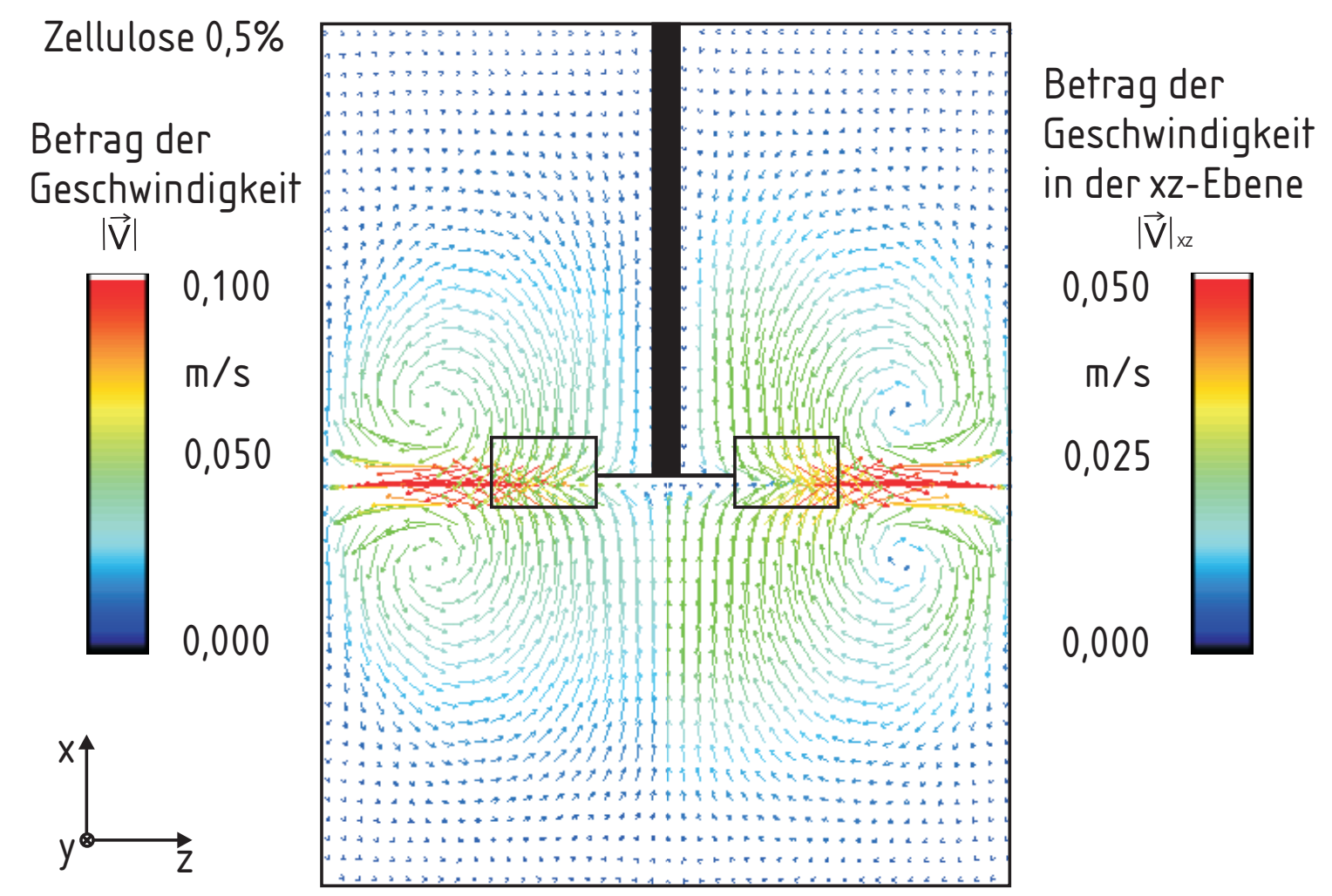

Bild 7.4: Geschwindigkeitsfeld in der Ebene zwischen den Rührerblättern, farbig codiert mit dem Betrag $|\vec{v}|$ bzw. $|\vec{v}|_{x z}$ für $\xi_{1}=0,5 \%$ Zellulose

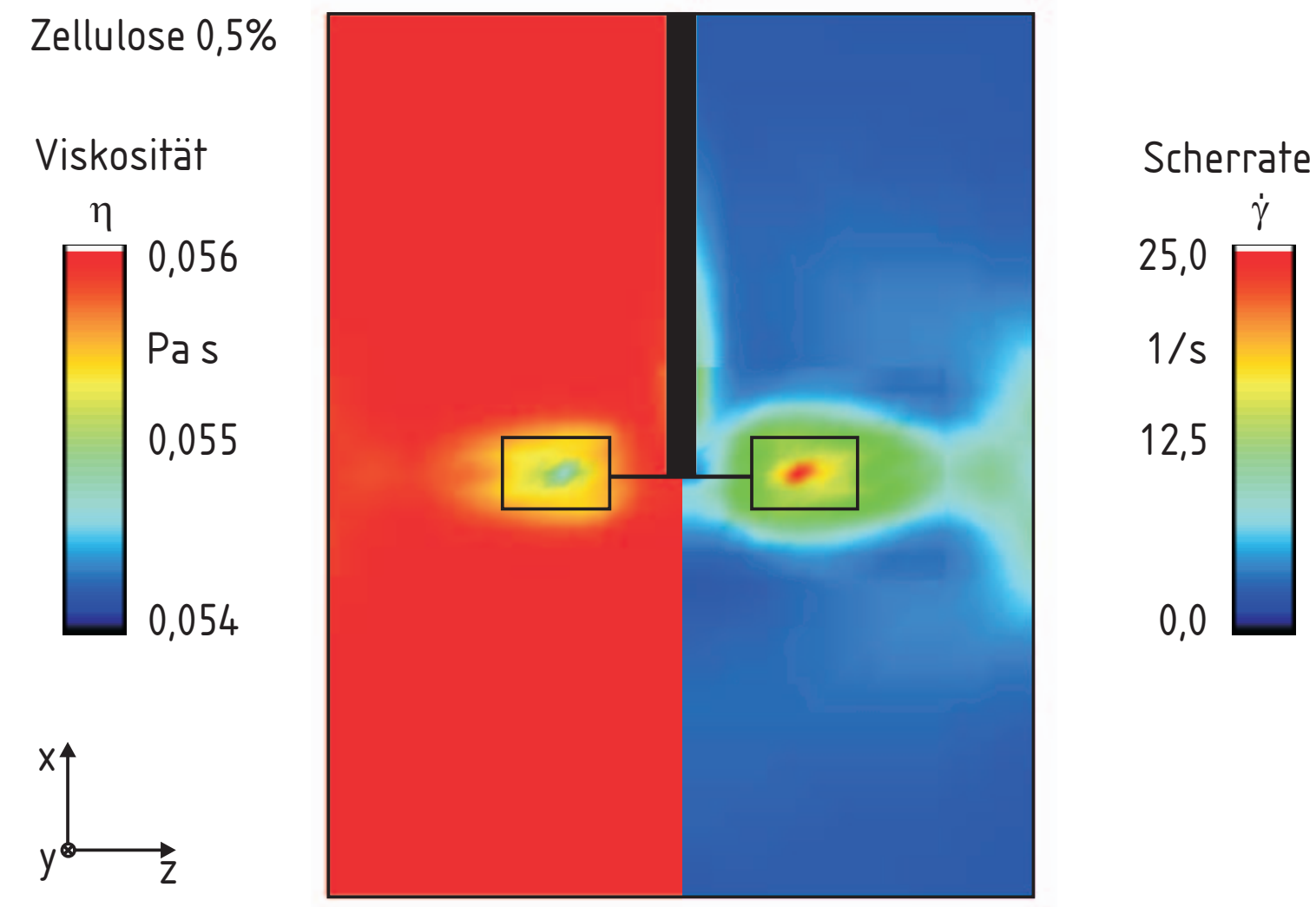

Bild 7.5: Viskosität $\eta$ und Scherrate $\dot{\gamma}$ in der Ebene zwischen den Rührerblättern für $\xi_{1}=0,5 \%$ Zellulose 
linken Bildhälfte ist der Gesamtbetrag des räumlichen Geschwindigkeitsvektors

$$
|\vec{v}|=\sqrt{v_{x}^{2}+v_{y}^{2}+v_{z}^{2}}
$$

dargestellt. Die maximale Geschwindigkeit wird demnach in der Rührerebene erreicht und beträgt etwa $0,1 \mathrm{~m} / \mathrm{s}$. Die Farbskala in der rechten Bildhälfte codiert den Betrag der Geschwindigkeitsvektoren projiziert auf die dargestellte x-z-Ebene

$$
|\vec{v}|_{x z}=\sqrt{v_{x}^{2}+v_{z}^{2}}
$$

Der Maximalwert der Geschwindigkeit $|\vec{v}|_{x z}$ wird wiederum in der Rührerebene erreicht, beträgt aber nur etwa $0,05 \mathrm{~m} / \mathrm{s}$. Im Zentrum des Ringwirbels gilt $|\vec{v}|_{x z}=0$, so dass dort ausschließlich eine Bewegung in Umfangsrichtung stattfindet.

Die Scherung der Fluidelemente ist hier deshalb sehr klein. Am Bifurkationspunkt an der Behälterwand, direkt an der Rührerwelle sowie in der Rührerebene sind hingegen Zonen sehr hoher Deformationen. Die repräsentative Deformationsgeschwindigkeit gemäß Gl.(7.25) ist in Bild 7.5 in der rechten Hälfte dargestellt. In direkter Umgebung der Rührerblätter beträgt sie etwa $\dot{\gamma}=25 \mathrm{~s}^{-1}$. Der überwiegende Teil des Behälters weist jedoch nur sehr geringe Deformationsgeschwindigkeiten auf. Der strukturviskose Charakter der Zelluloselösung mit einem Massenanteil von nur $\xi_{1}=0,5 \%$ ist kaum ausgeprägt. Die Viskosität der Lösung ist in der linken Hälfte von Bild 7.5 dargestellt. Sie ist im gesamten Behälter nahezu konstant und entspricht etwa der Nullviskosität $\eta_{0}=0,056 \mathrm{~Pa}$ s. Lediglich in Bereichen hoher Deformationsgeschwindigkeiten ist eine sehr geringe Abnahme der Viskosität auf etwa 0,054 Pa s zu erkennen.

In Bild 7.6 ist das Geschwindigkeitsfeld für die wässrige Zelluloselösung mit $\xi_{2}=1,0 \%$ Zellulose projiziert auf die Ebene zwischen den Rührerblättern dargestellt. Der Gesamtbetrag des Geschwindigkeitsvektor nach Gl. (7.27) ist wieder in der linken Bildhälfte farbig codiert. Er ist, bedingt durch die größere Umfangsgeschwindigkeit bei der Rührerdrehzahl $n_{r 2}=250 \mathrm{~min}^{-1}$, auf maximal $0,5 \mathrm{~m} / \mathrm{s}$ angestiegen. Auch in der Projektion auf die dargestellte x-z-Ebene ist der Maximalwert von $|\vec{v}|_{x z}$ nach Gl. (7.28) auf maximal 0,2 m/s angestiegen. Allerdings ist nun das Verhältnis

$$
v_{s e k}^{*}=\frac{\max \left(|\vec{v}|_{x z}\right)}{\max (|\vec{v}|)}
$$

von $v_{\text {sek }}^{*}=0,5$ für $\xi_{1}=0,5 \%$ auf $v_{\text {sek }}^{*}=0,44$ für $\xi_{2}=1,0 \%$ gesunken. Die Sekundärströmung ist also im Vergleich zur Gesamtströmung schwächer ausgeprägt. Dies ist auch an der geringeren geometrischen Ausdehnung der Ringwirbel zu erkennen.

Das strukturviskose Verhalten ist gegenüber der Zelluloselösung mit $\xi_{1}=0,5 \%$ deutlicher ausgeprägt. In Bild 7.7 ist im linken Teil die Viskositätsverteilung und im rechten Teil 


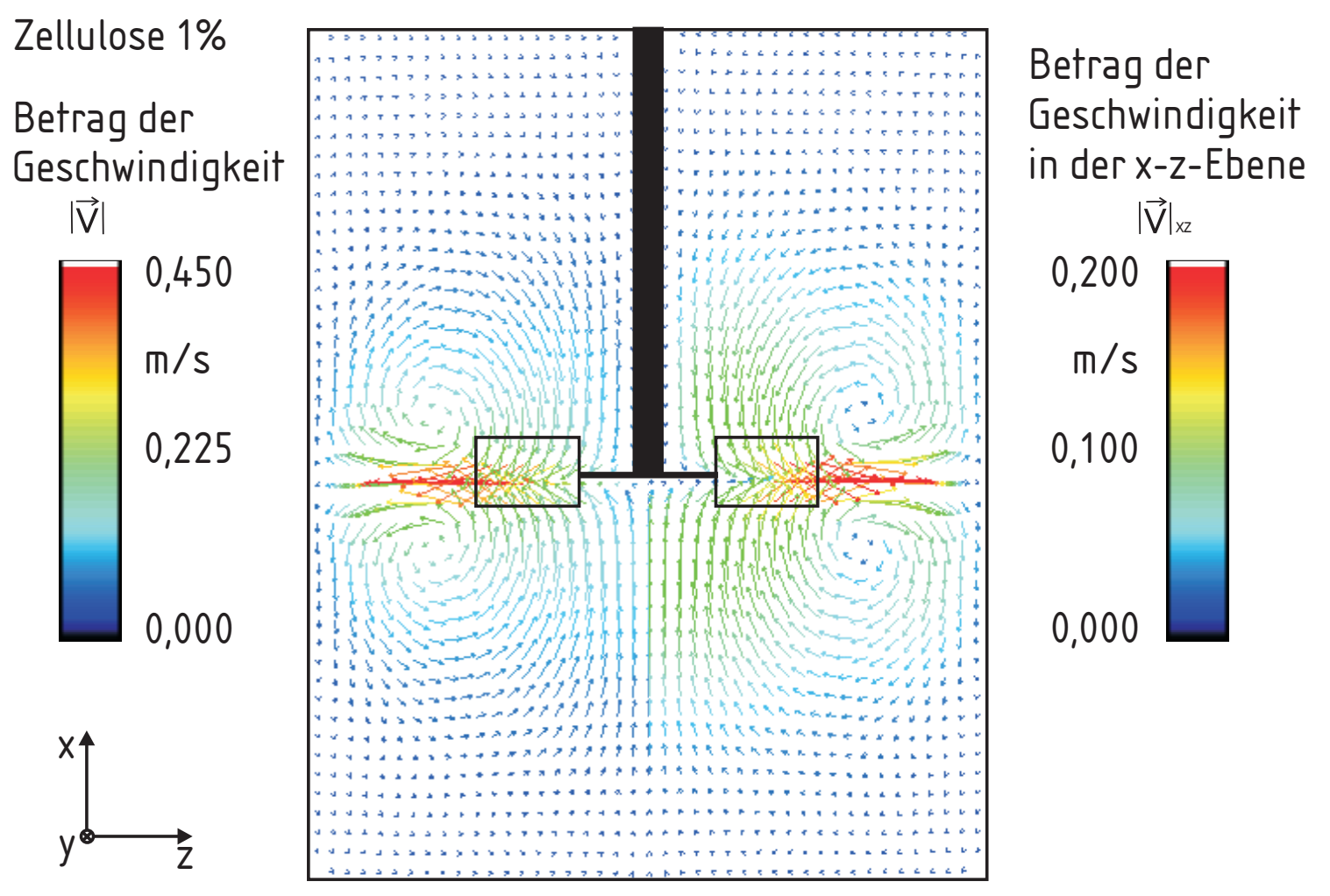

Bild 7.6: Geschwindigkeitsfeld in der Ebene zwischen den Rührerblättern, farbig codiert mit dem Betrag $|\vec{v}|$ bzw. $|\vec{v}|_{x z}$ für $\xi_{2}=1 \%$ Zellulose

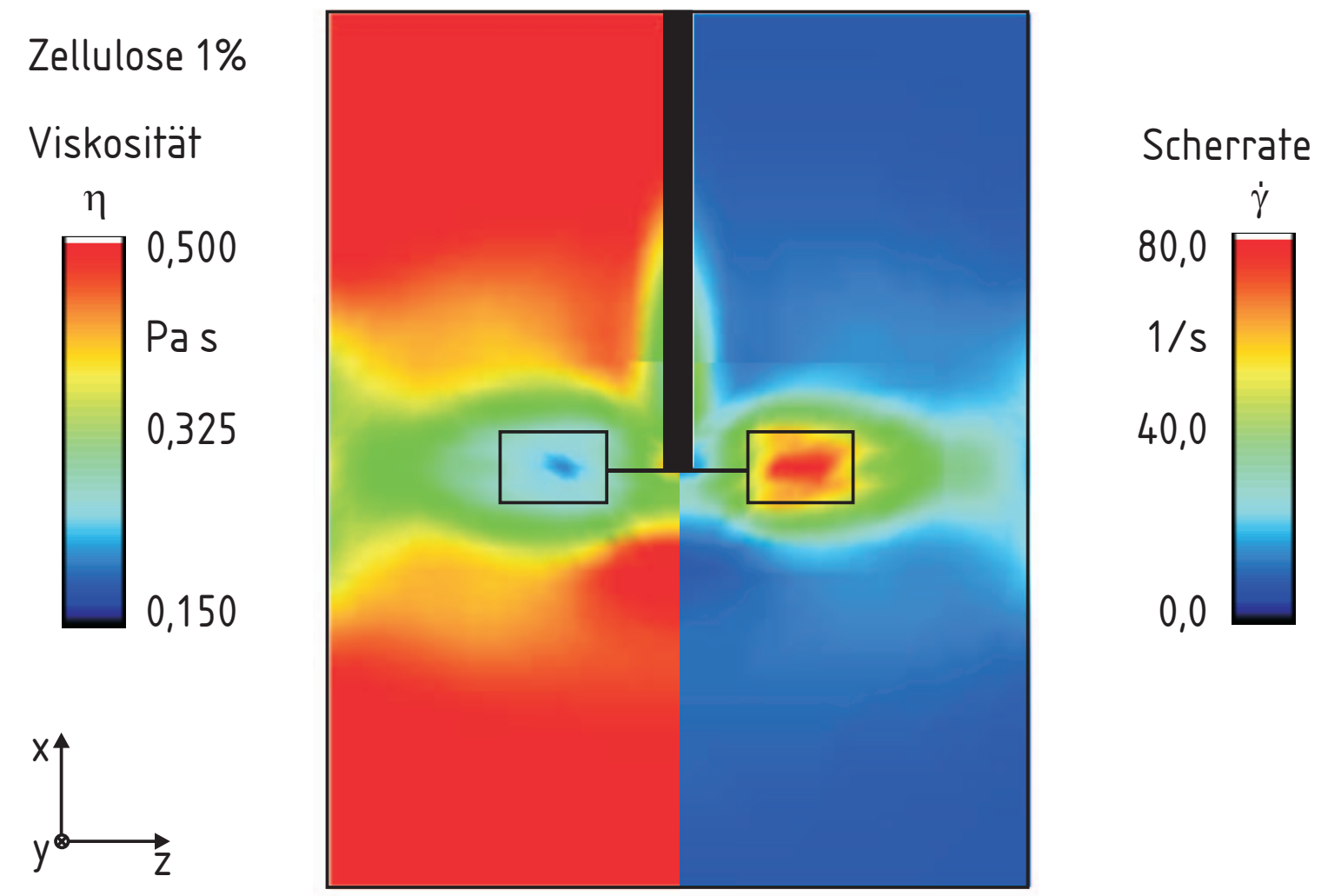

Bild 7.7: Viskosität $\eta$ und Scherrate $\dot{\gamma}$ in der Ebene zwischen den Rührerblättern für $\xi_{2}=1 \%$ Zellulose 
die Verteilung der repräsentativen Deformationsgeschwindigkeit dargestellt. In Bereichen hoher Deformation nahe der Rührerebene und direkt an der Rührerwelle nimmt die Viskosität bis auf etwa 0,15 Pa s ab. Im größten Teil des Behälters liegt jedoch etwa die Nullviskosität $\eta_{0}=0,52 \mathrm{~Pa}$ s vor. Der Betrag der Deformationsgeschwindigkeiten ist von $\dot{\gamma}=25 \mathrm{~s}^{-1}$ für die niedrigviskosere Zelluloselösung auf bis zu $\dot{\gamma}=80 \mathrm{~s}^{-1}$ gestiegen.

Für die am höchsten viskose Flüssigkeit mit $\xi_{3}=2,0 \%$ Zelluloseanteil ist eine Kavernenbildung zu erkennen. Als Kaverne wird der Bereich um die Rührerwelle bezeichnet, in der aufgrund der Abnahme der Viskosität in Bereichen mit hohen Deformationsgeschwindigkeiten eine gute Vermischung vorliegt. Außerhalb dieser Kaverne stagniert die Strömung, so dass nur eine geringe Vermischung stattfindet. In Bild 7.8 ist das Geschwindigkeitsfeld für die 2,0\%ige Zelluloselösung projiziert auf die Ebene zwischen den Rührerblättern dargestellt. Jeder Ringwirbel nimmt nur noch etwa ein Viertel des Behältervolumens ein. Das Zentrum des Ringwirbels ist gegenüber den niedrigviskoseren Fluiden weiter zur Behältermitte hin verschoben, und der Wirbel ist nahezu symmetrisch. Der Einfluss der Behälterwand hat deutlich abgenommen. Das Fluid wird schon vorher stark abgebremst, und die Geschwindigkeit im Bereich der Behälterwand ist nahezu Null. Der Betrag des Geschwindigkeitsvektors nach Gl. (7.27), links im Bild farbig codiert, ist aufgrund der hohen Drehzahl von $n_{r 3}=750 \mathrm{~min}^{-1}$ auf etwa $1,2 \mathrm{~m} / \mathrm{s}$ gestiegen. Auch in der Projektion auf die dargestellte x-z-Ebene ist der Maximalwert von $|\vec{v}|_{x z}$, rechts im Bild farbig codiert, auf maximal 0,4 m/s angestiegen. Im Verhältnis zur Gesamtströmung ist die Sekundärströmung allerdings nochmals schwächer geworden. Das Geschwindigkeitsverhältnis gemäß Gl. (7.29) beträgt nur noch $v_{\text {sek }}^{*}=0,33$. Mit zunehmender Viskosität nimmt also die Stärke der für die Vermischung wirksamen Sekundärströmung deutlich ab. Dies ist auch an der kleineren geometrischen Ausdehnung der Ringwirbel zu erkennen.

In Bild 7.9 ist im linken Teil wiederum die Viskositätsverteilung und im rechten Teil die Verteilung der repräsentativen Deformationsgeschwindigkeit dargestellt. Der Bereich hoher Scherraten ist nur noch in unmittelbarer Umgebung des Rührers zu finden. An der Behälterwand findet im Gegensatz zu den anderen beiden Zelluloselösungen kaum Scherung statt. Entsprechend bildet sich die oben erwähnte Kaverne geringer Viskosität um die Rührerebene aus, während am Boden und an der Flüssigkeitsoberfläche Viskositäten von bis zu 8 Pa s vorliegen. Der Maximalwert der repräsentativen Deformationsgeschwindigkeit hat deutlich auf etwa $\dot{\gamma}=300 \mathrm{~s}^{-1}$ zugenommen. 


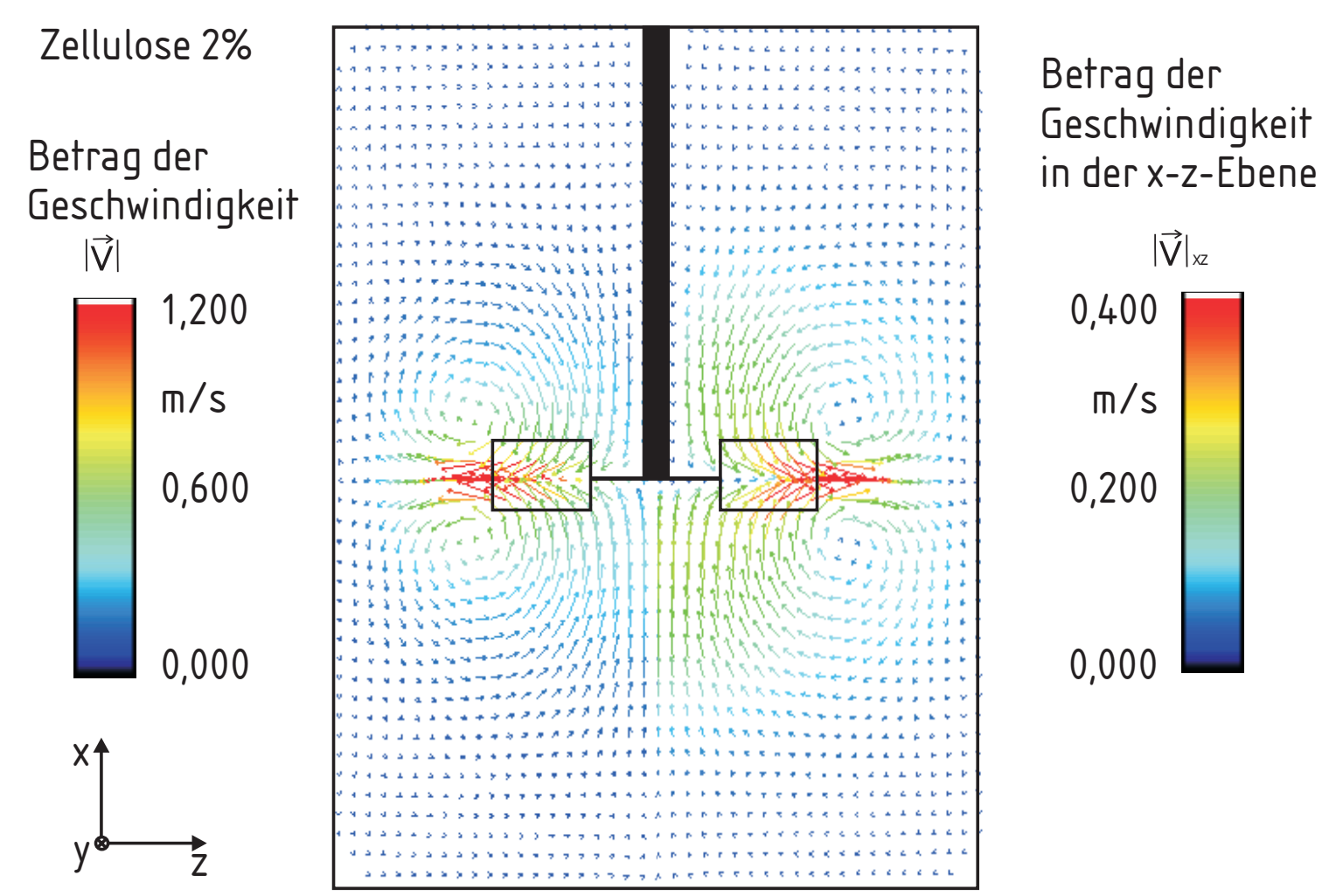

Bild 7.8: Geschwindigkeitsfeld in der Ebene zwischen den Rührerblättern, farbig codiert mit dem Betrag $|\vec{v}|$ bzw. $|\vec{v}|_{x z}$ für $\xi_{3}=2 \%$ Zellulose
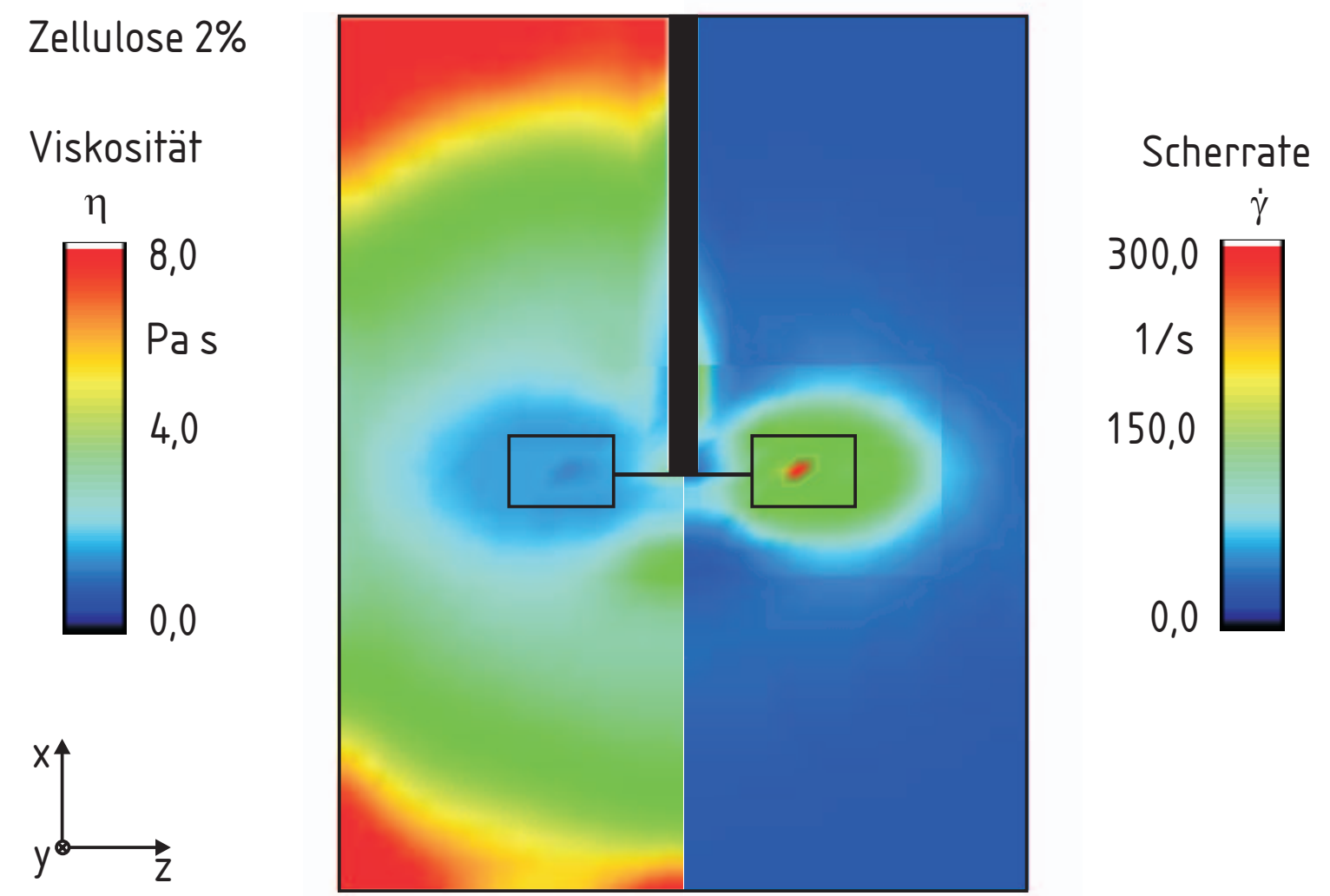

Bild 7.9: Viskosität $\eta$ und Scherrate $\dot{\gamma}$ in der Ebene zwischen den Rührerblättern für $\xi_{3}=2 \%$ Zellulose 


\section{Zwei-Farben Laserinduzierte Fluoreszenz}

Die Zwei-Farben Laserinduzierte Fluoreszenz ermöglicht das simultane Messen der zwei Farbstoffkonzentrationen in einer beliebigen Ebene des Rührbehälters.

\subsection{Optischer Aufbau}

Der optische Aufbau für die Messung mit der Zwei-Farben Laserinduzierten Fluoreszenz ist schematisch in Bild 8.1 dargestellt. Als Lichtquelle wird ein gepulster Festkörperlaser mit einer Frequenz von $30 \mathrm{~Hz}$ verwendet. Der Laser besteht aus einem Pumplaser (NewWave Nd:Yag, Tempest 30, 40 mJ/Puls), und einem daran angeschlossenen optischparametrischen Oszillator, kurz OPO (GWU OPO VisIr). Letzterer dient zur Frequenzumwandlung des Nd:Yag-Laser, so dass die Wellenlänge des emittierten Laserstrahls im sichtbaren Bereich zwischen $410 \mathrm{~nm}$ und $700 \mathrm{~nm}$ bzw. im infraroten Bereich zwischen $720 \mathrm{~nm}$ und $2600 \mathrm{~nm}$ frei wählbar ist. Für die nachfolgend beschriebenen Experimente wird die Wellenlänge $\lambda=495 \mathrm{~nm}$ eingestellt. Die Energie des emittierten Laserstrahls variiert stark mit der Wellenlänge. Bedingt durch den geringen Wirkungsgrad des OPOs von etwa 10 - 15\% und durch Verluste optischer Spiegelflächen etc. hat der emittierte Laserstrahl bei der Wellenlänge von $\lambda=495 \mathrm{~nm}$ eine Energie von etwa $2 \mathrm{~mJ} / \mathrm{Puls}$.

Der Laserstrahl wird mit Hilfe eines Strahlteilers etwa in einem Verhältnis von 9:1 geteilt. Der Strahl geringerer Leistung wird für eine Energiemessung des gepulsten Lasers verwendet. Diese Energiemessung ist in Kapitel 8.2.2 detailliert beschrieben. Der Laserstrahl höherer Leistung wird mit Hilfe eines Systems aus sphärischen und zylindrischen Linsen zu einem dünnen Lichtschnitt aufgeweitet. Der divergente Lichtschnitt beleuchtet eine beliebige horizontale oder vertikale Ebene im Rührbehälter, wobei der Fluoreszenzfarbstoff dort zum Leuchten angeregt wird. Das zylindrische Rührgefäß ist wie in Bild 8.2 dargestellt von einem rechteckigen Außengefäß umgeben. Dieses ist an allen Seiten, ausgenommen die der Kamera abgewandten Seite, mit Glasscheiben ausgestattet. Auf diese Weise ist der optische Zugang sowohl für einen vertikalen als auch für einen horizontalen Lichtschnitt gewährleistet. Die Aufnahme eines horizontalen Lichtschnitts erfolgt über einen Spiegel, der in einem Winkel von $45^{\circ}$ unter dem Behälter positioniert wird. Der Zwischenraum zwischen Außengefäß und Rührbehälter ist mit destilliertem Wasser gefüllt. Dieser Aufbau ermöglicht eine verzerrungsfreie Durchstrahlung des zylindrischen Rührgefäßes. Da der Laserstrahl senkrecht auf die Scheiben des Außengefäßes trifft, werden 


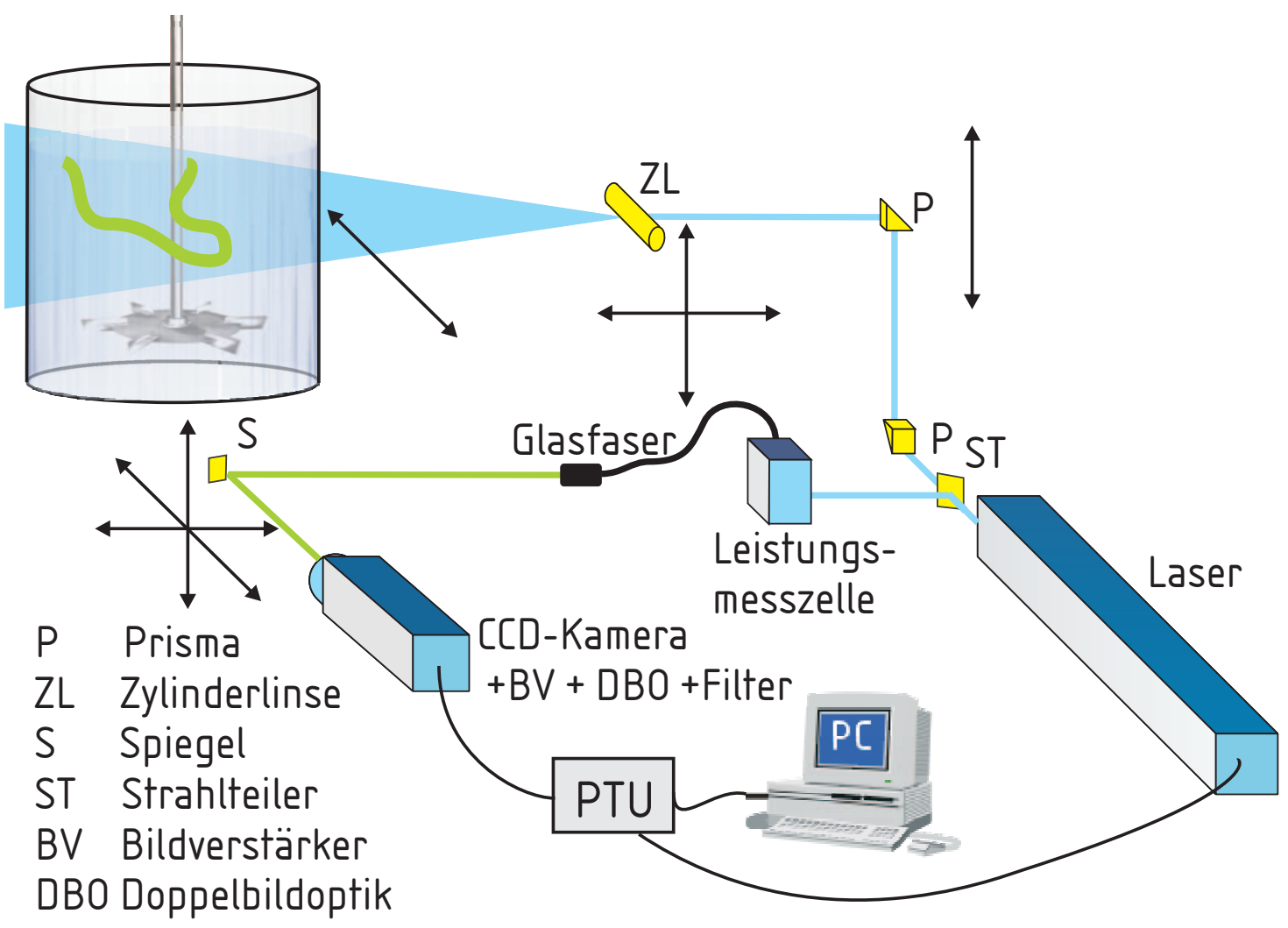

Bild 8.1: Optischer Aufbau für die Zwei-Farben Laserinduzierte Fluoreszenz

\section{Rührbehälter}

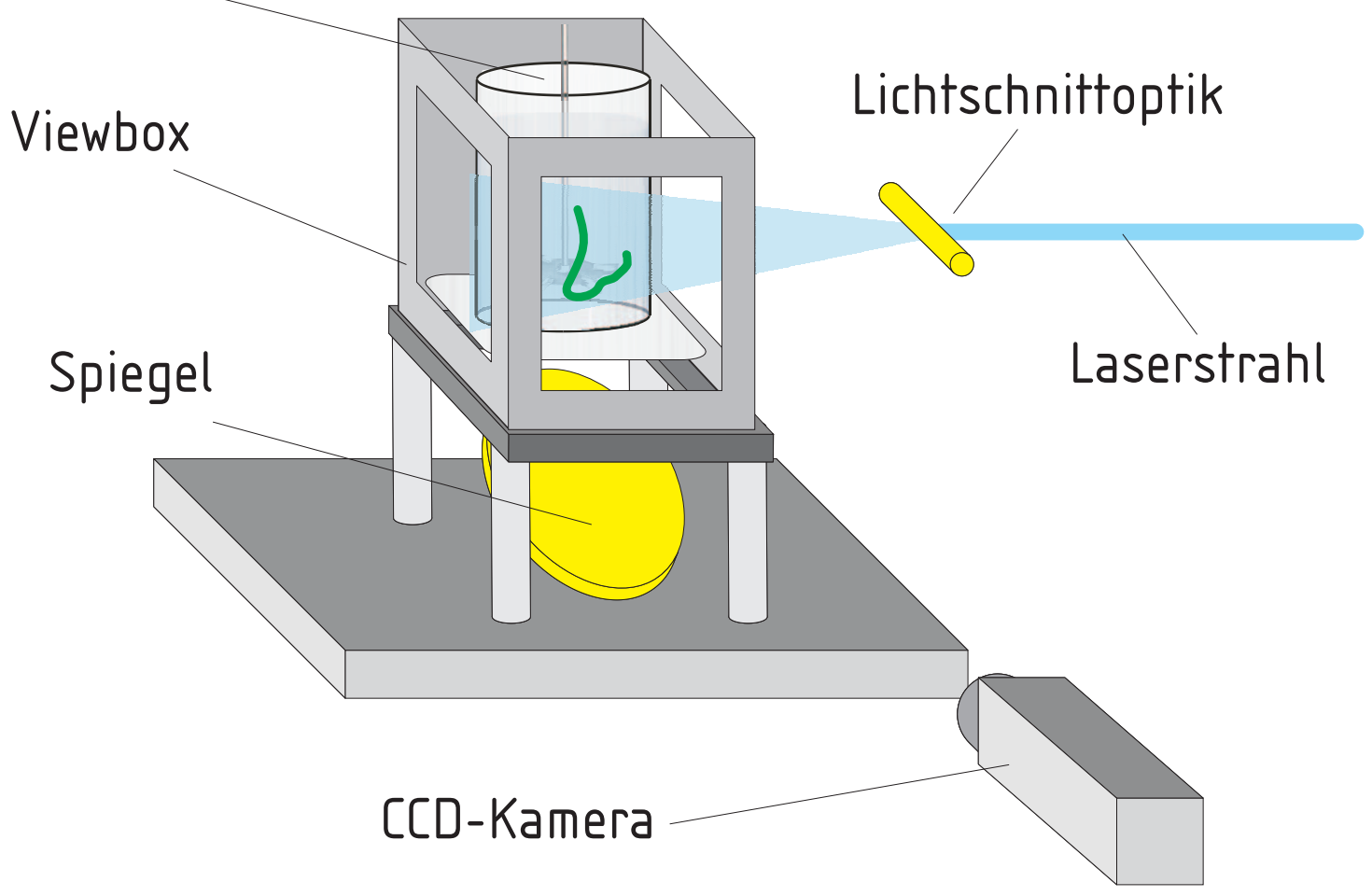

Bild 8.2: Positionierung des Rührbehälters im Messaufbau 
Reflexionen und Brechungen an den Glasscheiben minimiert.

Das emittierte Fluoreszenzlicht wird von einer CCD-Kamera (Imager 3, LaVision), die vertikal zur Messebene positioniert ist, detektiert. Die Intensität des einfallenden Lichts wird mit Hilfe eines Bildverstärkervorsatzes (IRO, LaVision) verstärkt. Die Nutzung eines Bildverstärkers hat zwei Vorteile: Zum einen können deutlich geringere Farbstoffkonzentrationen nachgewiesen werden (s. dazu Kapitel 8.2.4), zum anderen kann ein besseres Signalzu-Rausch-Verhältnis erzielt werden. Umgebungslicht und dadurch bedingte Reflexionen werden als Rauschen mit detektiert. Dieses ist um so größer, je länger die Belichtungszeit der Kamera ist. Diese ist zwischen $1 \mu \mathrm{s}$ und $1 \mathrm{~ms}$ frei wählbar, die Pulsdauer des Laserstrahls beträgt aber nur 5 ns. Berücksichtigt man die Abklingdauer der Fluoreszenz, so ist eine Belichtungszeit von 100 ns ausreichend, um das gesamte Fluoreszenzsignal zu detektieren. Mit Hilfe des Bildverstärkervorsatzes, der eine minimale Ausblendzeit von 7 ns hat, kann die Belichtungszeit genau auf den Laserpuls abgestimmt werden. Bildverstärker, Kamera und Laser werden von einem Computer gesteuert, wobei die Aufnahmefrequenz maximal $30 \mathrm{~Hz}$ beträgt. Für langsamere Mischvorgänge kann die Aufnahmefrequenz verringert werden. Die Fluoreszenzintensität wird in 12 bit Grauwerten gemessen, und die örtliche Auflösung des Kamerachips beträgt (640 x 480) Pixel.

Das Fluoreszenzlicht passiert zwei optische Filter (BP523 / 10 and RG645), die geeignet sind, die Fluoreszenzsignale der beiden Farbstoffe voneinander zu trennen. Eine Doppelbildoptik (LaVision) wird verwendet, um denselben Bildausschnitt zeitgleich zweimal aufnehmen zu können. Sie besteht aus zwei Aperturen, die mit den optischen Filtern ausgestattet werden, und einem System aus festen und drehbar gelagerten Spiegeln. Das Fluoreszenzlicht wird von den Spiegeln so auf den Kamerachip gelenkt, dass dort derselbe Bildausschnitt nebeneinander abgebildet wird, wobei jeweils in einer Hälfte idealerweise nur die Fluoreszenzintensität einer der beiden Farbstoffe gemessen wird. Für eine exakte Abbildung des Bildausschnitts in beiden Bildhälften ist eine Kalibrierung notwendig, die in Kapitel 8.2.1 beschrieben wird. Ein Foto der Doppelbildoptik vor Bildverstärker und CCDKamera ist in Bild 8.3 zu sehen. Zwischen der Doppelbildoptik und dem Bildverstärker ist ein Objektiv (Sigma, $\mathrm{f}=70 \mathrm{~mm}$ bis $210 \mathrm{~mm}, 1: 3,5 \sim 4,5$ ) mit variabler Brennweite geschaltet, so dass unterschiedliche Bildausschnitte sehr flexibel eingestellt werden können. Eine zusätzliche Makrolinse sorgt für kleine Abstände (etwa 1,5 m) zwischen Kamera und Objekt.

Mit dem Beginn der Kameraaufnahme wird auch die Farbstoffzufuhr in den Behälter gestartet. Die Eingabe in das Rührgefäß erfolgt über eine Glaskapillare, die an dem Außengefäß des Rührbehälters befestigt werden kann. Sie ist über einen Schlauch mit einer Dosiervorrichtung verbunden. Die Vorrichtung (s. Bild 8.4) besteht aus einer Spritze, deren Kolben von einer Gewindestange mit Schrittmotorantrieb verfahren wird. Diese Anord- 


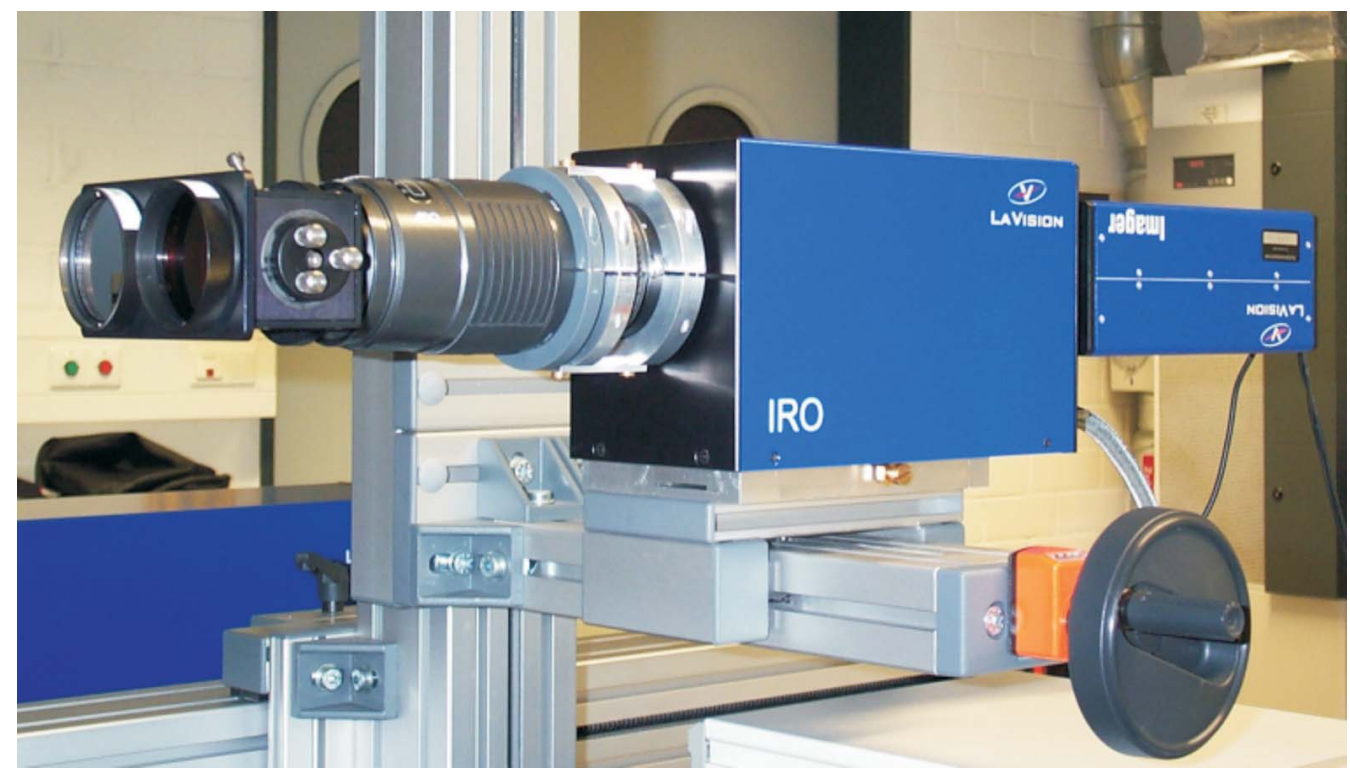

Bild 8.3: Foto von der CCD-Kamera mit Bildverstärkervorsatz, Doppelbildoptik und optischen Filtern

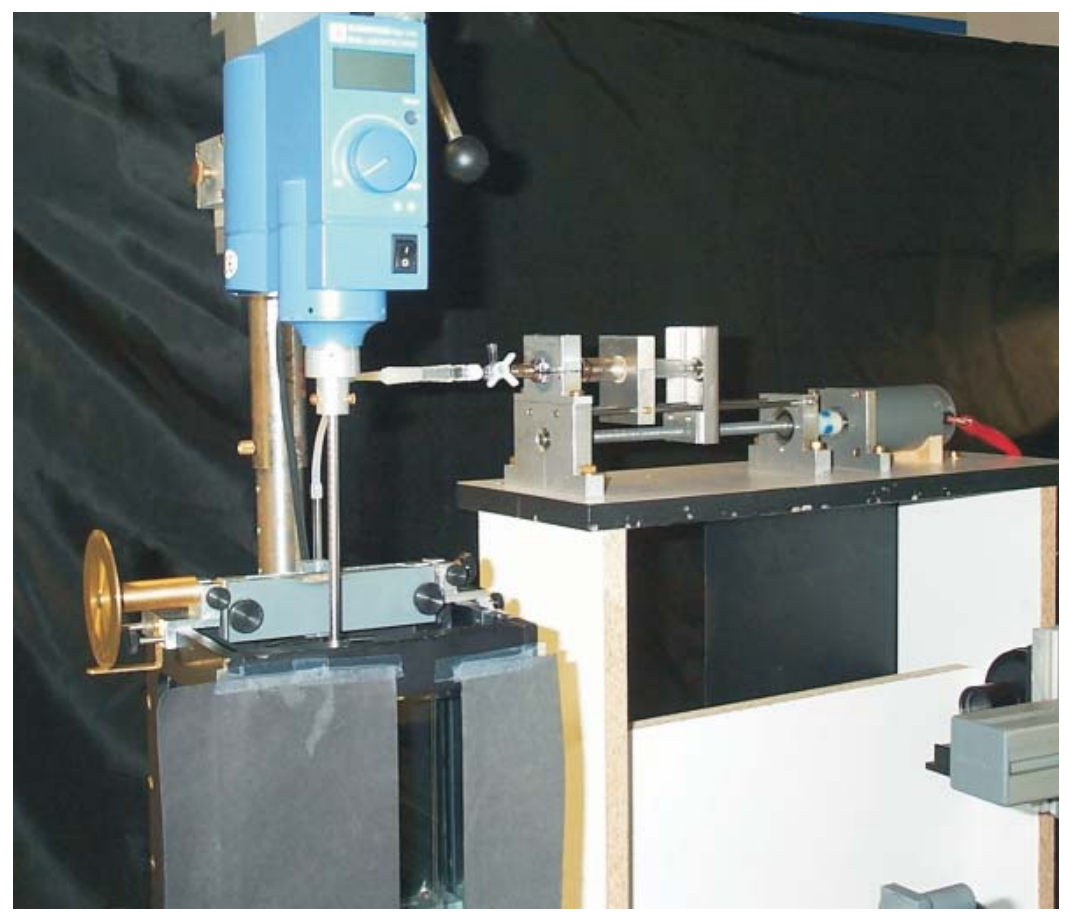

Bild 8.4: Foto der Dosiervorrichtung für die Fluoreszenzfarbstofflösung 
nung ermöglicht eine genau definierte Zugabemenge und -geschwindigkeit. Der maximale Volumenstrom beträgt 1,25 ml/s. Falls nicht anders angegeben, wird $1 \mathrm{ml}$ des Farbstoffgemisches mit einem Volumenstrom von $0,5 \mathrm{ml} / \mathrm{s}$ in den Behälter dosiert. Ausgenommen der Position direkt unterhalb des Rührers kann der Farbstoff an jeder beliebigen Stelle in das Rührgefäß dosiert werden.

Der Rührbehälter, die Kamera und die Lichtschnittoptik sind jeweils auf einer Linearführung montiert. Auf diese Weise können unterschiedliche Ebenen im Behälter beleuchtet und der entsprechende Bildausschnitt reproduzierbar eingestellt werden. Durch Variieren der Entfernung zwischen Lichtschnittoptik und Rührbehälter kann die Größe der beleuchteten Ebene an die des gewählten Bildausschnitts angepasst werden, da der Lichtschnitt mit einem Winkel von $2,7^{\circ}$ divergiert.

\subsection{Kalibrierung des Messsystems}

\subsubsection{Doppelbildoptik}

Mit Hilfe einer Doppelbildoptik (LaVision) ist es möglich, bei Verwendung nur einer Kamera denselben Bildausschnitt zeitgleich zweimal aufzunehmen. Die Doppelbildoptik besteht aus zwei Aperturen, die mit den optischen Filtern ausgestattet werden, und einem System aus festen und drehbar gelagerten Spiegeln. In Bild 8.5 ist der Strahlengang der Fluoreszenzstrahlung innerhalb der Doppelbildoptik schematisch dargestellt. Das Fluoreszenzlicht wird von den Spiegeln so auf den Kamerachip gelenkt, das dort derselbe Bildausschnitt nebeneinander abgebildet wird.

Da der Bildausschnitt durch zwei Aperturen, die einen kleinen Abstand voneinander haben, betrachtet wird, ist der Blickwinkel unterschiedlich. Der Fehler, der durch diese schräge Betrachtung des Bildausschnitts entsteht, kann durch eine entsprechende Kalibrierung korrigiert werden. Der vollständige Ablauf dieser Kalibrierung ist schematisch in Bild 8.6 dargestellt. Vor Beginn der Versuche wird eine mit Kreuzen markierte Platte an der Stelle im Rührbehälter positioniert, die während der Experimente vom Laser beleuchtet wird. Die Kalibrierplatte wird mit Hilfe einer Tischlampe beleuchtet und auf dem Kamerachip abgebildet. Dabei wird zunächst die rechte Apertur abgedeckt und die beweglichen Spiegel der Doppelbildoptik so eingestellt, dass der gewünschte Bildausschnitt in der linken Bildhälfte zu sehen ist. Da durch jede Apertur der Doppelbildoptik der gesamte Kamerachip belichtet wird, muss die rechte Bildhälfte von einer nicht strahlenden, schwarzen Blende abgedeckt werden. Diese wird entsprechend vor dem Rührbehälter positioniert. In einem zweiten Schritt wird jetzt die linke Apertur der Doppelbildoptik abgedeckt und derselbe Bildausschnitt durch Einstellen der Spiegel und Positionieren 


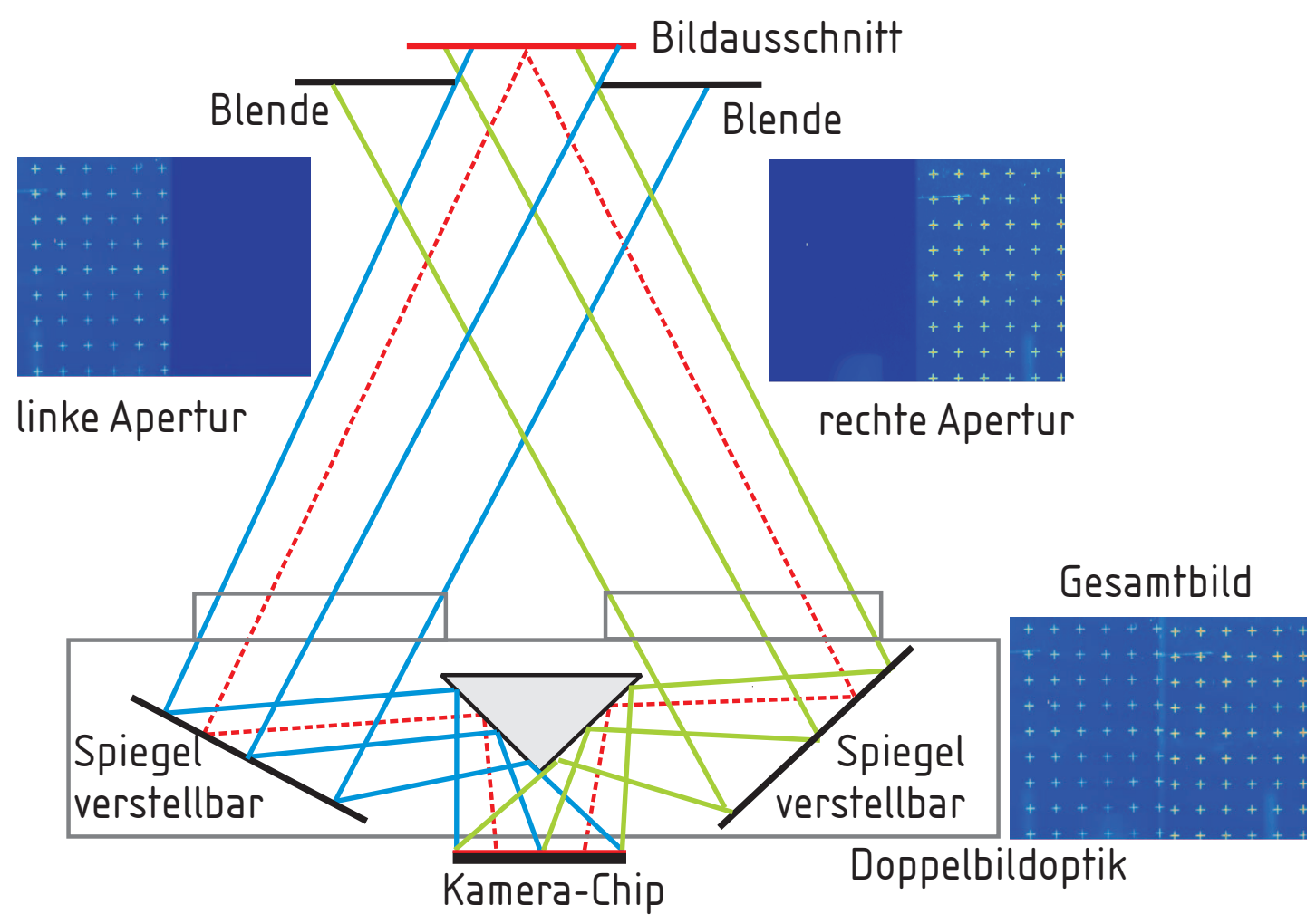

Bild 8.5: Strahlengang in der Doppelbildoptik
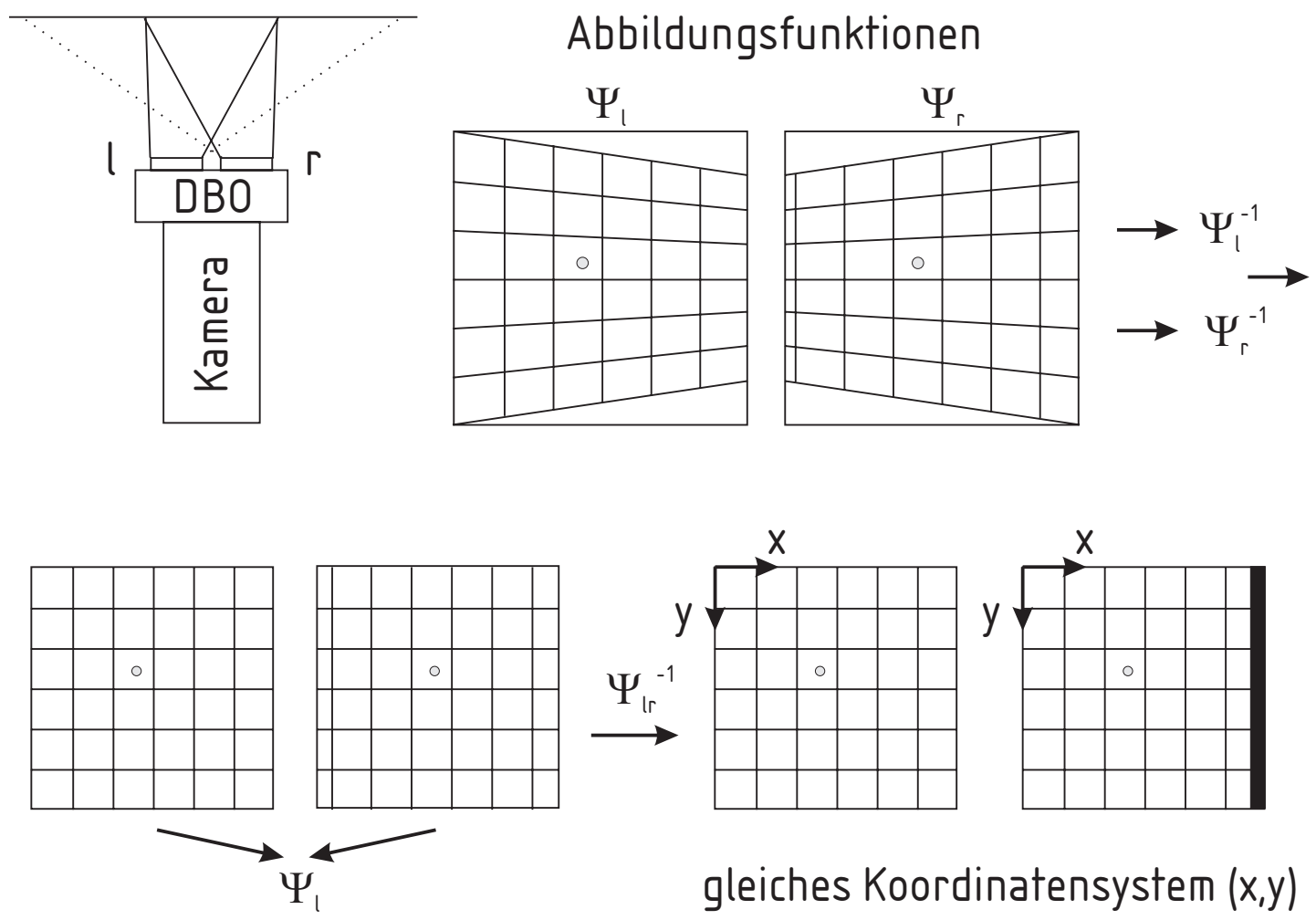

Bild 8.6: Schematischer Ablauf der Kalibrierung der Doppelbildoptik 
einer weiteren Blende auf der rechten Bildhälfte abgebildet. Zur Orientierung bei der Einstellung des Bildausschnitts dient eine spezielle Markierung auf der Kalibrierplatte. Wie Bild 8.5 zu entnehmen ist, wird bei geöffneten Aperturen das Bild des gewünschten Bildausschnitts, das mit der linken Apertur detektiert wurde, mit dem Bild der (strahlungslosen) Blende, das mit der rechten Apertur detektiert wurde, überlagert und andersherum. Für die Kalibrierung werden alle Kreuze auf der Platte sowohl im linken als auch im rechten Bildteil detektiert. Die Abstände der Markierungen sind konstant und bekannt. Somit ist es möglich, eine Abbildungsfunktion $\Psi$ aus dem wahren Abstand $s_{G}$ und dem abgebildeten Abstand $s_{B}$ der Kreuze zu berechnen. Dies wird sowohl für den linken $\left(\Psi_{l}\right)$ als auch für den rechten Bildteil $\left(\Psi_{r}\right)$ durchgeführt. Durch Anwenden der inversen Abbildungsfunktionen $\left(\Psi_{l}^{-1}\right)$ bzw. $\left(\Psi_{r}^{-1}\right)$ wird der Bildausschnitt unverzerrt dargestellt. Aus dem bekannten Abstand der Kreuze und der Größe eines Pixels $s_{P}$ wird ein Abbildungsmaßstab

$$
\text { Res }=\frac{s_{G}}{s_{B}} s_{P}
$$

berechnet. Dieser gibt an, welche Länge auf einen Pixel des Kamerachips abgebildet wird. Damit den beiden Bildausschnitten auch exakt dasselbe Koordinatensystem zugrunde liegt, wird nach der Korrektur der schiefen Abbildung ein Vergleich der beiden Bildhälften durchgeführt. Dabei dient erneut die spezielle Markierung auf der Kalibrierplatte zur Orientierung. Mit Hilfe einer Kreuzkorrelation der beiden Bildteile wird eine weitere Ab-

bildungsfunktion $\Psi_{l r}$ berechnet. Nach Anwenden der inversen Abbildungsfunktion $\Psi_{l r}^{-1}$ auf beide Bildhälften liegt ihnen dasselbe Koordinatensystem zugrunde.

\subsubsection{Messung der Energie gepulster Laser}

Für die Beleuchtung der Messebene im Rührbehälter, die dort zur Anregung der Fluoreszenzfarbstoffe führt, wird wie oben beschrieben ein gepulster Laser verwendet. Da die Energie des Lasers von Puls zu Puls variiert, muss sie zeitgleich zu den Messungen im Rührbehälter auf getrenntem Wege gemessen werden. Andernfalls besteht kein eindeutiger Zusammenhang zwischen der Intensität des vom Farbstoff emittierten Fluoreszenzlichts und seiner Konzentration, wie sie in Gl. (5.19) angegeben ist. Der effektive Proportionalitätsfaktor $m=K \Phi I_{0} \varepsilon s$ ist von der Laserleistung $I_{0}$ abhängig.

Für die Messung der Laserenergie wird ein kleiner Teil des Laserstrahls mit Hilfe eines Strahlteilers ausgekoppelt und in die in Bild 8.7 schematisch dargestellte Messzelle umgelenkt. Diese enthält eine mit Fluoreszenzfarbstoff (carboxy-SNARF, $c_{2}=3,11 \cdot 10^{-6} \mathrm{~mol} / \mathrm{l}$ ) gefüllte Küvette. Der Laserstrahl beleuchtet den Farbstoff, und das Fluoreszenzlicht wird in ein um $90^{\circ}$ versetzt zum Laserstrahl angeordnetes Glasfaserkabel eingekoppelt. Der 


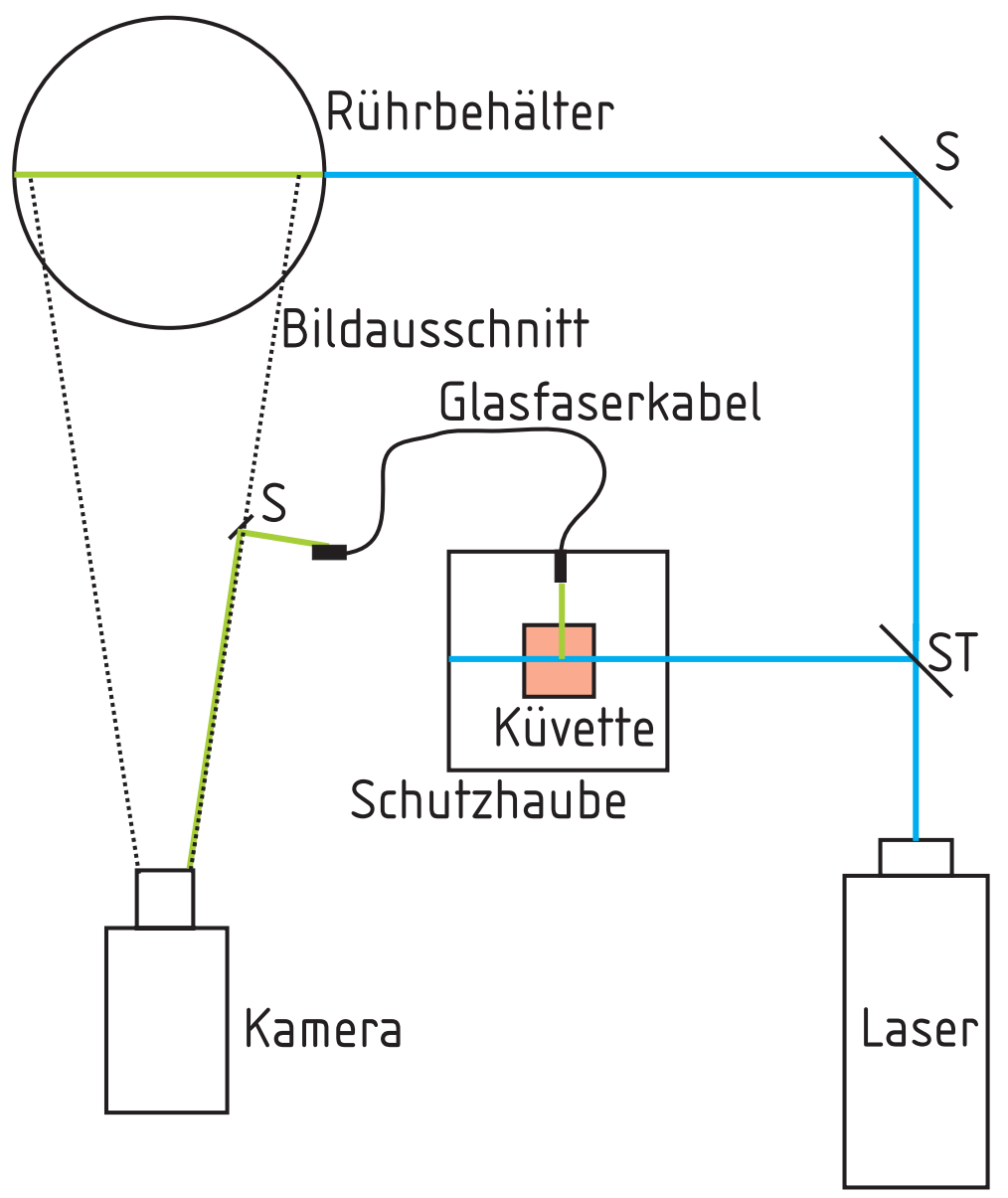

Bild 8.7: Optischer Aufbau für die Energiemessung des gepulsten Lasers

ausgekoppelte Strahl beleuchtet einen kleinen Spiegel, der so positioniert ist, dass der Lichtpunkt der Glasfaser scharf auf dem Kamerachip abgebildet wird. Dadurch geht dieser Bereich des Kamerabildes für die Aufnahme der Mischung im Rührbehälter verloren. Allerdings ist der Leuchtpunkt im allgemeinen (je nach Vergrößerung) nur wenige Pixel groß. Außerdem entfällt eine aufwendige Triggerung eines externen Energiemessgeräts mit dem Laser und eine zusätzliche Speicherung und Zuordnung der Messwerte. Jedes Bild enthält automatisch die Information über die Pulsenergie des Lasers zum Zeitpunkt der Bildaufnahme.

Dabei ist nicht die tatsächliche Energie entscheidend, es wird vielmehr ein Referenzwert $\bar{e}_{a v g}$ ermittelt. Dazu wird aus einem zeitlich über eine bestimmte Anzahl von Laserpulsen $\bar{n}$ gemittelten Bild der Mittelwert der Fluoreszenzintensität im Bereich des Lichtpunkts berechnet. Im allgemeinen ist eine Mittelung über $\bar{n}=200$ bis 400 ausreichend. Für jedes aufgenommene Bild $n$ wird im gleichen Bereich des Leuchtpunkts der Mittelwert der Fluoreszenzintensität $e_{\text {avg }}(n)$ bestimmt. Das Ergebnis ist in Bild 8.8 dargestellt. Es sind deutlich die Schwankungen der Laserenergie zu sehen. Ähnliche Schwankungen zeigt auch das Fluoreszenzsignal $K(x, y)$ einer homogenen Farbstofflösung, die im Rührbehälter vorgelegt ist. Der Mittelwert über alle Pixel $(\mathrm{x}, \mathrm{y})$ des Bildes, $k_{\text {avg }}(n)$, ist ebenfalls in Bild 8.8 


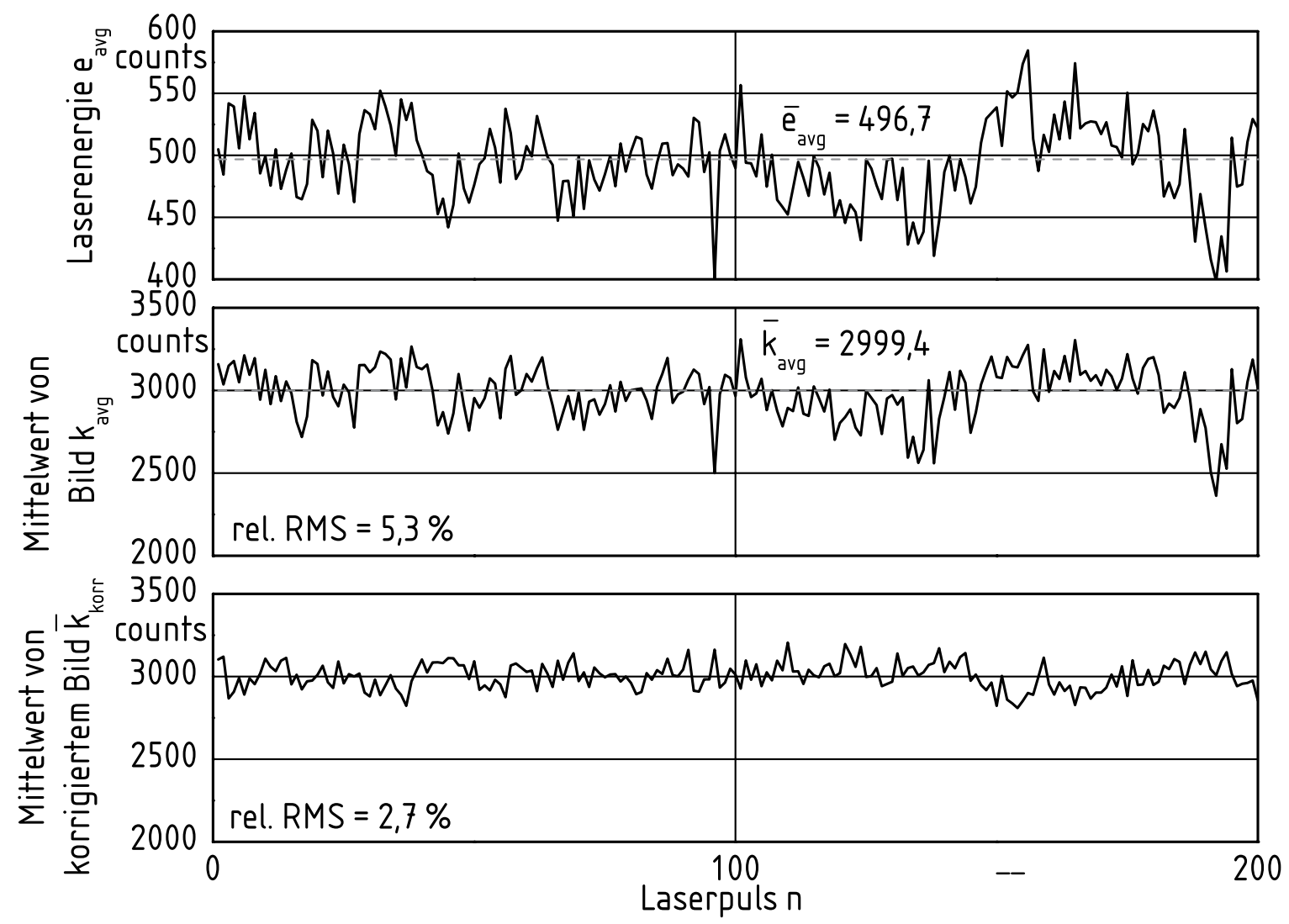

Bild 8.8: Schwankungen der Laserenergie und Korrektur der Messwerte

dargestellt. Die relative Standardabweichung vom Mittelwert $\bar{k}_{a v g}=2999,4$ counts beträgt 5,3\%. Die Korrektur dieses Bildes erfolgt nun nach der folgenden Beziehung:

$$
K_{k o r r}(n)=K(n) \frac{\bar{e}_{\text {avg }}}{e_{\text {avg }}(n)}
$$

Der durch die Schwankungen der Laserenergie hervorgerufenen Messfehler kann durch diese Korrektur etwa halbiert werden, wie der Mittelwert des korrigierten Signals $k_{a v g, k o r r}(n)$ unten in Bild 8.8 deutlich zeigt.

\subsubsection{Korrektur des Intensitätsprofils}

Die Energie ist über dem Laserstrahl nicht gleichmäßig verteilt, sondern es ist eine deutliche Überhöhung im Zentrum des Strahls zu erkennen. Die Verteilung ist in etwa ein rotationssymmetrisches, abgeflachtes Gaußprofil. Durch die Aufweitung des Laserstrahls zu einem dünnen Lichtschnitt stellt sich ein verzerrtes Profil ein. Wird eine homogen vermischte Farbstofflösung beleuchtet, so kann das in Bild 8.9 dargestellte Fluoreszenzsignal $K(x, y)$ gemessen werden. Bei der Messung wurden 200 Bilder zeitlich gemittelt. Im Verlauf des Laserstrahls (x-Koordinate) ist quasi keine Veränderung feststellbar. Dies deutet 


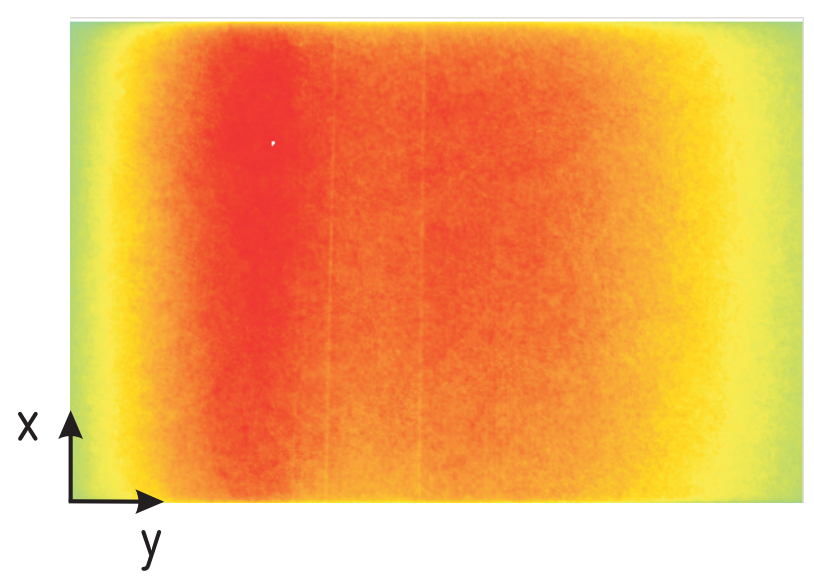

Bild 8.9: Fluoreszenz-Intensitätsprofil in der Lichtschnittebene

darauf hin, dass die Konzentration der Farbstofflösung so gering ist, dass die Absorption entlang des Strahlwegs vernachlässigbar ist. Quer zur Richtung der Strahlausbreitung (y-Koordinate) ist jedoch ein deutliches Profil zu erkennen. In Bild 8.10 ist der Verlauf der Fluoreszenzintensität $i_{F}$ als Funktion der Bildhöhe y für einen konstanten x-Wert als schwarze Linie dargestellt. Es ist ein leicht unsymmetrischer Verlauf zu erkennen, wobei der Maximalwert im Zentrum etwa 152 counts und der Minimalwert am Rand etwa 84 counts beträgt. Die Asymmetrie ist Folge diverser Strahlumlenkungen und damit verbundenen Rückreflexionen an den Glasflächen der zur Umlenkung verwendeten Prismen sowie einer leicht schiefen Beleuchtung der zur Aufweitung des Laserstrahls verwendeten Zylinderlinse. Die Standardabweichung um den Mittelwert 126 counts beträgt 17 counts, was eine relative Streuung von 13,5\% bedeutet. Würde dieses Signal direkt für die Berechnung des Konzentrationsfeldes der (in diesem Falle homogenen) Farbstofflösung verwendet, so würde ein erheblicher Fehler entstehen. Aus dem Bild der homogenen Farbstofflösung $K(x, y)$ wird deshalb die Korrekturmatrix

$$
M(x, y)=\frac{\bar{k}}{K(x, y)}
$$

berechnet. Dabei ist $\bar{k}$ der Mittelwert über einen geeigneten Bildausschnitt:

$$
\bar{k}=\frac{1}{n m} \sum_{i=1}^{n} \sum_{j=1}^{m} K\left(x_{i}, y_{j}\right) .
$$

Um den dynamischen Bereich der Kamera voll ausnutzen zu können, sollte der Bildausschnitt im Zentrum des Bildes liegen, so dass $\bar{k}$ etwa dem Maximalwert entspricht. Die Korrektur erfolgt nun, indem die detektierten Bilder mit der Korrekturmatrix multipliziert werden:

$$
K_{k o r r}(x, y)=K(x, y) \cdot M(x, y)
$$

Der Verlauf der korrigierten Fluoreszenzintensität $i_{F}$ als Funktion der Bildhöhe y ist für den gleichen $\mathrm{x}$-Wert als graue Linie in Bild 8.10 dargestellt. Die relative Standardab- 


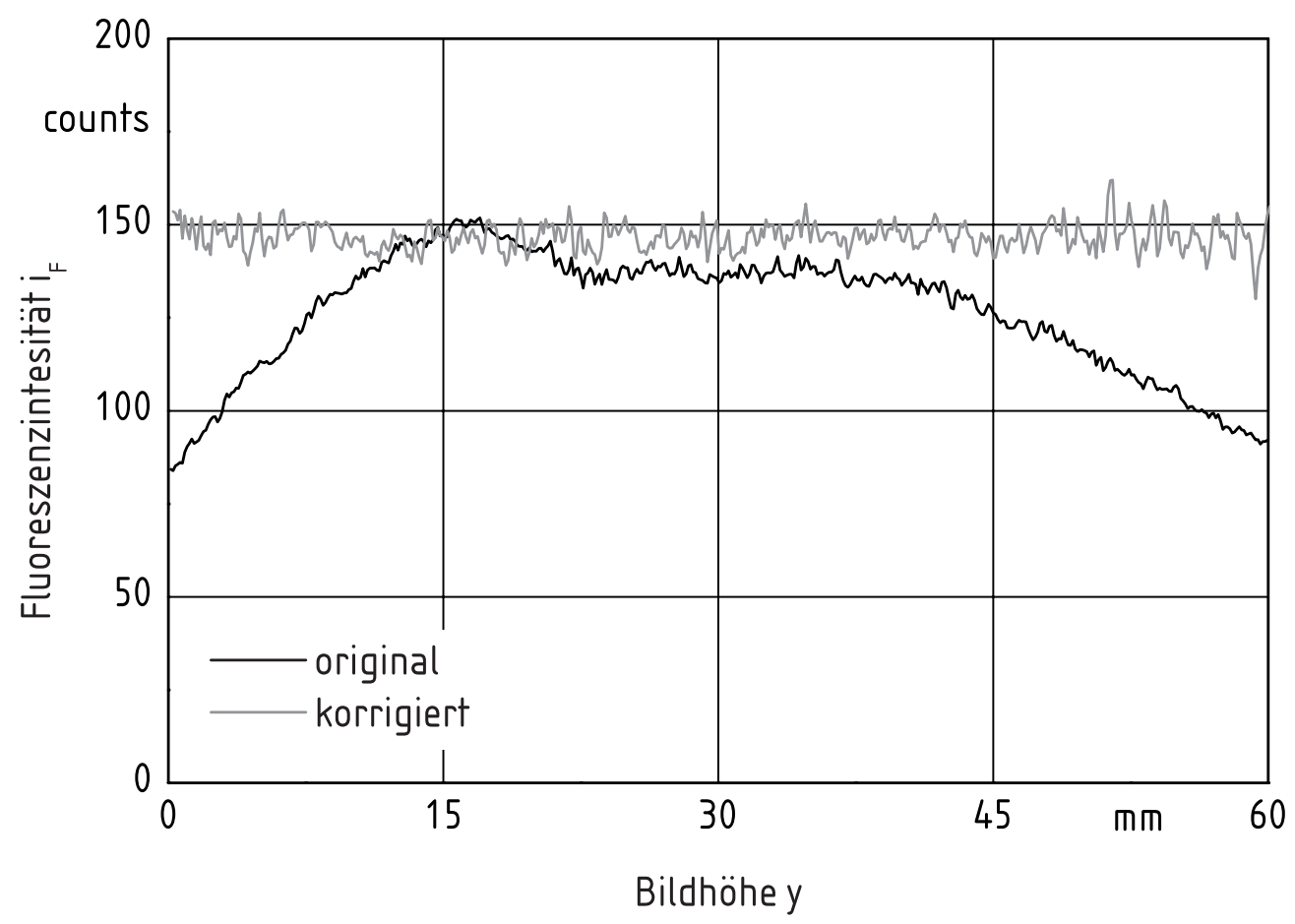

Bild 8.10: Fluoreszenzintensität über der Bildhöhe vor und nach der Korrektur

weichung um den Mittelwert 147 counts beträgt nur noch 2,6\%, so dass eine erhebliche Reduzierung des Fehlers erzielt werden kann.

\subsubsection{Fluoreszenzintensität und Konzentration}

Damit aus den gemessenen Fluoreszenzemissionen die Konzentrationen der Farbstoffe berechnet werden können, ist das Messsystem gemäß Gl. (5.19) zu kalibrieren, d.h. der effektive Proportionalitätsfaktor $m$ muss bestimmt werden. Dabei ist allerdings zu beachten, dass die von der Kamera detektierte Fluoreszenzintensität nur dann eindeutig der Konzentration $c$ des Fluoreszenzfarbstoffs zugeordnet werden kann, wenn nur ein Fluoreszenzfarbstoff vorhanden ist. Wird zeitgleich ein zweiter Farbstoff zur Fluoreszenz angeregt, so können die Absorptions- und Emissionsspektren, wie in Kapitel 6.1 beschrieben, überlappen und die Farbstoffe sich dementsprechend gegenseitig beeinflussen. Zunächst soll deshalb geklärt werden, wie groß der Messfehler ist, der durch das Überlappen des Absorptionsspektrums von carboxy-SNARF mit dem Emissionsspektrum von fluo-4 ist.

In Kapitel 6.1 ist hergeleitet, welcher Anteil des emittierten Fluoreszenzlichts des Farbstoffs 1 von Farbstoff 2 reabsorbiert wird. Gleichung (6.6) ist zu entnehmen, dass Versuchsparameter wie die Konzentration des Farbstoffs 2 (carboxy-SNARF) und die Durchstrahlungslänge in der Lösung vor dem Detektor einen großen Einfluss haben. In Bild 8.11 
ist der mit Gl. (6.8) berechnete insgesamt absorbierte Anteil

$$
I_{F 1, \text { norm }}^{*}=\int_{\lambda=\lambda_{\text {ex }}}^{\lambda=\infty} i_{F 1, \text { norm }}^{*}(\lambda) \mathrm{d} \lambda
$$

als Funktion der Farbstoffkonzentration mit der Lauflänge als Parameter dargestellt. Es werden im Folgenden zwei repräsentative Parametersätze untersucht:

1. Der Farbstoff liegt zu Beginn der Vermischung hochkonzentriert vor, die Abmessungen der Lamellen sind gering.

2. Der Farbstoff liegt zum Ende der Vermischung homogenisiert im gesamten Behälter vor.

Im ersten Fall wird die Anfangskonzentration $c_{2}=c_{2,0} \approx 2 \cdot 10^{-6} \mathrm{~mol} / \mathrm{l}$ gewählt. Außerdem wird angenommen, dass die maximale Ausdehnung dieser hochkonzentrierten Bereiche $l=0,025 d_{r}=2,38 \mathrm{~mm}$ beträgt. Wie in Kapitel 9.4 gezeigt, entspricht das im Mittel etwa zwei bis drei Lamellendicken. In diesem Fall wird die Fluoreszenzintensität von fluo-4 um weniger als 2,5\% reduziert, der Fehler ist somit vernachlässigbar. Dies gilt auch für eine doppelt so große Ausdehnung von $l=0,05 d_{r}=4,75 \mathrm{~mm}$. Aufgrund der schnellen Verdünnung während der Mischung kann dieser Wert als oberes Limit angesehen werden.

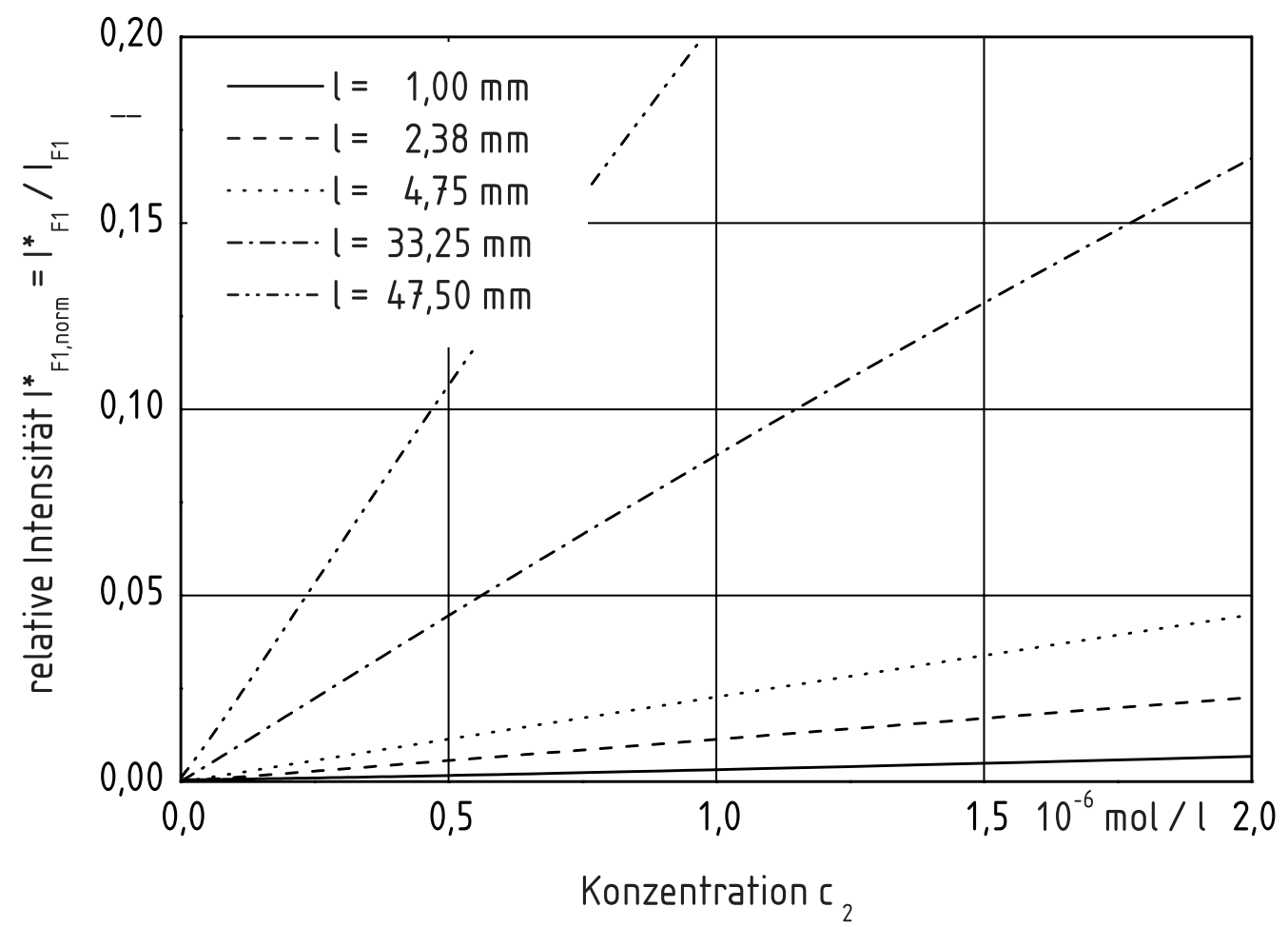

Bild 8.11: Relative Abnahme der Fluoreszenzintensität von fluo-4 aufgrund von Reabsorption durch carboxy-SNARF 
Im zweiten Fall wird die Konzentration $c_{2}=1,6 \cdot 10^{-8} \mathrm{~mol} / \mathrm{l}$ gewählt. Diese ergibt sich, wenn acht Versuche nacheinander durchgeführt werden, wobei jeweils $1 \mathrm{ml}$ der Farbstofflösung mit $c_{2,0}=2 \cdot 10^{-6} \mathrm{~mol} / \mathrm{l}$ hinzudosiert werden und im Behälter homogen verteilt werden. Üblicherweise werden höchstens fünf Versuche mit derselben Flüssigkeit im Behälter durchgeführt. Die Durchstrahlungslänge ist nun maximal der halbe Behälterdurchmesser $l=0,5 d_{r}=47,5 \mathrm{~mm}$. In diesem Fall wird die Fluoreszenzintensität von fluo-4 um weniger als 0,4 \% reduziert. Der Beitrag der homogen verteilten Farbstofflösung ist so gering, dass auch bei Addition der Absorptionseffekte der Fehler vernachlässigbar ist. Es wird deshalb keine Korrektur vorgenommen. Auch in der Praxis hat sich gezeigt, das die Fluoreszenzintensität von fluo-4 nicht messbar geringer ist, wenn sie in der oben beschriebenen Umgebung einer carboxy-SNARF- Lösung anstatt in einer wässrigen Umgebung gemessen wird.

Neben der Reabsorption des Fluoreszenzlichts durch den zweiten Farbstoff senkrecht zum Laserstrahl ist eine Absorption in Ausbreitungsrichtung möglich. Für homogene Lösungen hoher Konzentration ist deshalb eine deutliche Abschattung in Strahlrichtung zu beobachten. Analog zu Gl. (6.8) wird das Verhältnis

$$
I_{A, \text { norm }}=\frac{I_{A}\left(c_{1}, c_{2}, s\right)}{I_{0}}=1-e^{-\left(\varepsilon_{1} c_{1}+\varepsilon_{2} c_{2}\right) s}
$$

aus der entlang des Laserstrahls auf der Weglänge $s$ absorbierten Strahlung $I_{A}$ und der Laserintensität $I_{0}$ berechnet. In Bild 8.12 ist die relative Absorption in Abhängigkeit der Konzentration von carboxy-SNARF dargestellt. Dabei wird entsprechend der in Kapitel 9 dargestellten experimentellen Ergebnisse ein konstantes Konzentrationsverhältnis $c_{1} / c_{2}=0,464$ angenommen. Für die Anregungswellenlänge des Lasers $\lambda=495 \mathrm{~nm}$ beträgt der Extinktionskoeffizient des Farbstoffs carboxy-SNARF $\varepsilon_{2}=19894 \mathrm{l} /(\mathrm{mol} \mathrm{cm})$ und der Extinktionskoeffizient des Farbstoffs fluo-4 $\varepsilon_{1}=201396$ l/(mol cm). Letzteres gilt für den vollständig abreagierten Fall, so dass die Ergebnisse als oberes Limit gelten können. Für den ersten Grenzfall mit $c_{2}=2 \cdot 10^{-6} \mathrm{~mol} / \mathrm{l}$ und $s=2,38 \mathrm{~mm}$ ergibt sich eine Absorption von etwa $5 \%$. Für eine homogene Konzentration von $c_{2}=1,6 \cdot 10^{-8} \mathrm{~mol} / \mathrm{l}$ auf einer Strahllänge von $s=47,5 \mathrm{~mm}$ beträgt die Absorption weniger als 0,9\%. Der Fehler ist somit etwas größer als für die Reabsorption durch den zweiten Farbstoff, aber immer noch vernachlässigbar gering. Im Gegensatz zu dem Fehler für die Reabsorption durch den zweiten Farbstoff wäre eine Korrektur aber dann möglich, wenn der Bildausschnitt so gewählt ist, dass der Eintritt des Laserstrahls in den Behälter abgebildet wird.

Die überlappenden Emissionsspektren der Fluoreszenzsignale werden mit Hilfe optischer Filter voneinander getrennt. In der Praxis ist aber im allgemeinen keine perfekte Trennung möglich, so dass Filter $I$ nicht nur für Fluoreszenzlicht von Farbstoff 1 sondern auch für Fluoreszenzlicht von Farbstoff 2 durchlässig ist, und außerdem Filter II nicht nur 


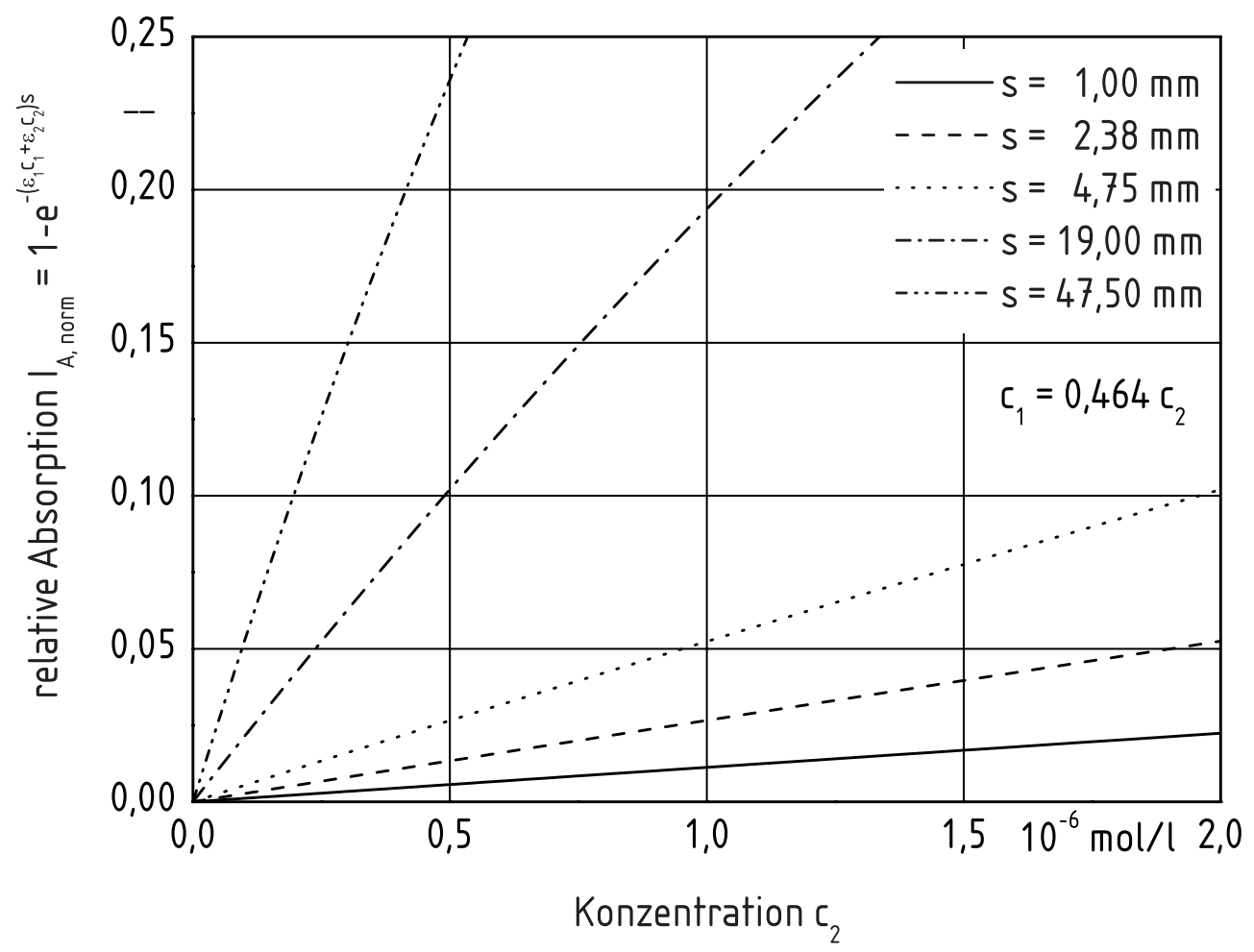

Bild 8.12: Relative Absorption in Ausbreitungsrichtung des Laserstrahls

für Fluoreszenzlicht von Farbstoff 2 sondern auch für Fluoreszenzlicht von Farbstoff 1 durchlässig ist. In diesem Fall kann das von der Kamera durch Filter I bzw. II detektierte Fluoreszenzlicht $I_{F}^{I}$ und $I_{F}^{I I}$ wie folgt beschrieben werden

$$
\begin{aligned}
I_{F}^{I} & =\alpha_{1}^{I} I_{F 1}+\alpha_{2}^{I} I_{F 2} \\
I_{F}^{I I} & =\alpha_{1}^{I I} I_{F 1}+\alpha_{2}^{I I} I_{F 2} .
\end{aligned}
$$

Dabei sind $\alpha_{1}^{I}$ und $\alpha_{1}^{I I}$ die Anteile des Fluoreszenzlichts $I_{F 1}$ von Farbstoff 1, die durch den Filter $I$ bzw. $I I$ detektiert werden, für $\alpha_{2}^{I}$ und $\alpha_{2}^{I I}$ gilt dies analog. Diese Faktoren $\alpha$ müssen experimentell für die verwendeten Filter bestimmt werden. Dabei entsprechen $\alpha_{1}^{I}=K_{1}$ und $\alpha_{2}^{I I}=K_{2}$ dem vom Messsystem abhängigen Parameter $K$ aus Gl. (5.18). Für das in dieser Arbeit verwendete Filtersystem aus BP 523/ FWHM 10 (Index I) und RG 645 (Index $I I$ ) sind außerdem die folgenden Vereinfachungen zulässig:

- Die Transmissionskurve für den Bandpaßfilter $I$ ist so eng, dass nur Fluoreszenzlicht von Farbstoff 1, nicht aber von Farbstoff 2 detektiert wird.

- Das durch Filter II detektierte Fluoreszenzlicht von Farbstoff 1 lässt sich als ein Bruchteil des Fluoreszenzlichts, das durch Filter $I$ detektiert wird, ausdrücken.

Mit den daraus folgenden Annahmen

$$
\alpha_{1}^{I}=K_{1} \Rightarrow \alpha_{1}^{I} I_{F 1}=I_{F 1, \text { detekt }}
$$




$$
\begin{aligned}
\alpha_{2}^{I} & =0 \\
\alpha_{1}^{I I} & =f^{I I} \alpha_{1}^{I} \\
\alpha_{2}^{I I} & =K_{2} \Rightarrow \alpha_{2}^{I I} I_{F 2}=I_{F 2, \text { detekt }}
\end{aligned}
$$

lässt sich Gl. (8.8) wie folgt vereinfachen

$$
\begin{aligned}
I_{F}^{I} & =I_{F 1, \text { detekt }} \\
I_{F}^{I I} & =f^{I I} I_{F}^{I}+I_{F 2, \text { detekt }} .
\end{aligned}
$$

Für die Bestimmung des Filterfaktors $f^{I I}$ wird die Fluoreszenzintensität einer Lösung bestehend nur aus dem Farbstoff fluo-4 $\left(c_{1}, c_{2}=0\right)$ gemessen. Dann gilt $I_{F 2 \text {,detekt }}=0$ und

$$
f^{I I}=\frac{I_{F}^{I I}}{I_{F}^{I}} .
$$

Für das verwendete Filter- und Farbstoffsystem konnte ein Filterwert von $f^{I I} \approx 0,1$ gemessen werden.

Der Zusammenhang zwischen der Fluoreszenzintensität $I_{F 1 \text {,detekt }}$ und der Konzentration $c_{1}$ sowie der zwischen der Fluoreszenzintensität $I_{F 2 \text {,detekt }}$ und der Konzentration $c_{2}$ muss ebenfalls experimentell bestimmt werden. Dazu werden Farbstofflösungen unterschiedlicher Konzentration hergestellt, die jeweils nur einen der beiden Farbstoffe enthalten und die zugehörige Fluoreszenzemissionen gemessen. Dann gelten die Beziehungen $I_{F}^{I}=I_{F 1 \text {,detekt }}\left(c_{1}, c_{2}=0\right)$ und $I_{F}^{I I}=I_{F 2 \text {,detekt }}\left(c_{1}=0, c_{2}\right)$, und die Messsignale $I_{F}^{I}$ und $I_{F}^{I I}$ können direkt verwendet werden.

Während der Kalibriermessungen müssen die gleichen Einstellungen gewählt werden, die während der Mischexperimente gewählt sind. Dazu gehört, dass Bildausschnitt und Verstärkungsgrad des Bildverstärkers übereinstimmen. Da der Rührbehälter ein Volumen von etwa 11 fasst, wäre eine sehr große Menge an Farbstoff für diese Kalibrierung notwendig. Aus Kostengründen wird darauf verzichtet und statt dessen nur jeweils ein Volumen von $30 \mathrm{ml}$ hergestellt. Die Farbstofflösungen werden in kleine zylindrische Glasbehälter gefüllt, die so in dem rechteckigen Glasbehälter platziert werden, dass der Lichtschnitt die Symmetrieebene beleuchtet. Auf diese Weise entstehen durch die unterschiedlichen Krümmungen der Glaswände keine Abbildungsfehler.

In Bild 8.13 sind die gemessenen Fluoreszenzintensitäten $I_{F 1 \text {,detekt }}$ und $I_{F 2 \text {,detekt }}$ doppellogarithmisch über der Konzentration der Farbstoffe aufgetragen. Diese Form der Auftragung ist sinnvoll, da die Konzentrationen sehr gering sind und bei einer linearen Auftragung die Messwerte sehr dicht beieinander liegen würden. Die logarithmische Form der Darstellung hat außerdem den Vorteil, dass eine lineare Abhängigkeit der Form $y=m \cdot x$ durch eine Gerade mit der Steigung 1 dargestellt wird. Geraden mit unterschiedlichem 


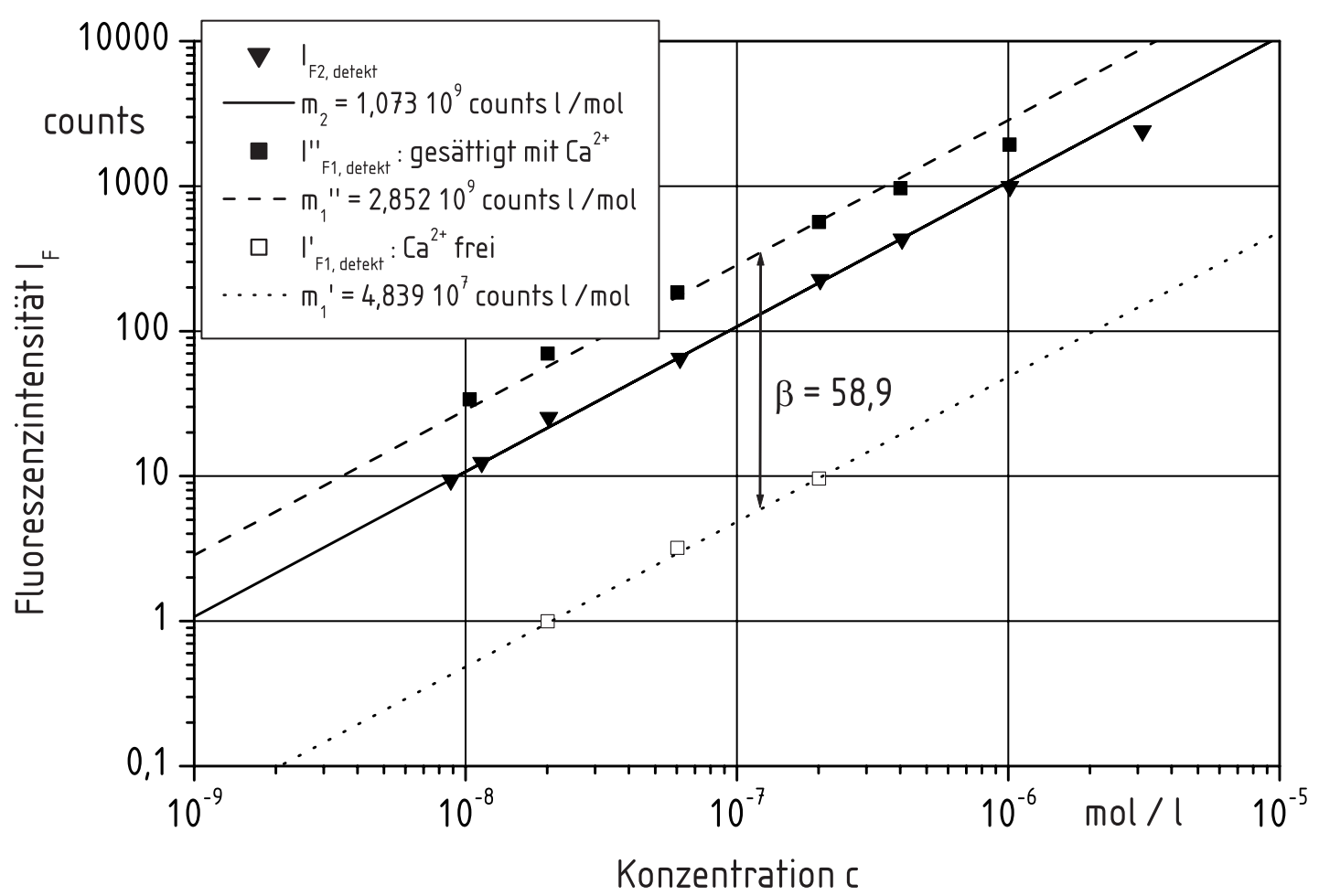

Bild 8.13: Integrale Fluoreszenzintensität als Funktion der Farbstoffkonzentration

Proportionalitätsfaktor $m$ werden also als parallel verschobene Geraden mit der gleichen Steigung 1 abgebildet.

Für den inerten Farbstoff carboxy-SNARF werden die Fluoreszenzintensitäten für acht unterschiedliche Konzentrationen gemessen. Dabei wird jeweils ein Mittelwert über 200 bis 400 Bilder in einem repräsentativen Gebiet in dem Glasbehälter ermittelt.

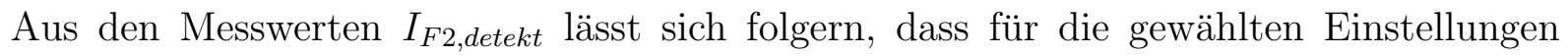
des Messsystems der lineare Bereich der Fluoreszenzemission bis zur Konzentration $c_{2} \approx 1 \cdot 10^{-6} \mathrm{~mol} / \mathrm{l}$ reicht. Die Gerade $I_{F 2}=m_{2} \cdot c_{2}$, die die Messwerte bis zu dieser Konzentration am besten annähert, ist ebenfalls in Bild 8.13 dargestellt. Der Parameter $m_{2}$ wird durch Minimieren der Fehlerquadratsumme zu $m_{2}=1,07268 \cdot 10^{9}$ (counts l) $/ \mathrm{mol}$ berechnet. Für kleine Konzentrationen weichen die Messwerte (mit Ausnahme des Messpunkts für $\left.c_{2}=2,03 \cdot 10^{-8} \mathrm{~mol} / \mathrm{l}\right)$ um maximal $4 \%$ von dieser Geraden ab. Für größere Konzentrationen $\left(c_{2}>2 \cdot 10^{-6} \mathrm{~mol} / \mathrm{l}\right)$ ist die Absorption entlang des Strahlwegs im Messvolumen so groß, dass ein degressiver Verlauf zu beobachten ist. Die Abweichungen von der Linearität beträgt nun etwa $28 \%$ an, so dass der Zusammenhang

$$
c_{2}=\frac{I_{F 2, \text { detekt }}}{m_{2}}
$$

nicht mehr gilt.

Für den reagierenden Farbstoff fluo-4 muss jeweils eine Konzentrationsreihe für den mit 
Calciumionen gesättigten Zustand des Farbstoffs $I_{F 1 \text {,detekt }}^{\prime \prime}$ und eine für die Calcium-freie Lösung $I_{F 1 \text {,detekt }}^{\prime}$ gemessen werden. Gegenüber carboxy-SNARF zeigt fluo-4 im gesättigten Zustand eine leicht erhöhte Fluoreszenzemission. Allerdings ist schon ab einem Wert von $c_{1} \approx 5 \cdot 10^{-7} \mathrm{~mol} / \mathrm{l}$ eine Abweichung von der Linearität zu verzeichnen. Für kleinere Konzentrationen weichen die Messwerte (mit Ausnahme des Messpunkts für $c_{1}=2,0 \cdot 10^{-8} \mathrm{~mol} / \mathrm{l}$ ) um maximal $15 \%$ von der Ausgleichsgerade ab, danach werden relative Abweichungen von über 30\% gemessen. Die minimal detektierbare Konzentration beträgt etwa $10^{-9} \mathrm{~mol} / \mathrm{l}$ (s. Kapitel 5.3), so dass ein Konzentrationsbereich von fast drei Zehnerpotenzen gemessen werden kann.

Für die Calcium-freie Lösung zeigt sich ebenfalls eine lineare Abhängigkeit der Fluoreszenzemission von der Konzentration des Farbstoffs fluo-4. Die relative Abweichung der Messwerte beträgt zwischen 1\% und 9\%. Damit ergibt sich ein von der Konzentration des Farbstoffs unabhängiger Verstärkungsfaktor $\beta$ (s. Gl.(6.13)). Dieser kann aus den Proportionalitätsfaktoren

$$
m_{1}=\frac{I_{F 1, \text { detekt }}^{\prime \prime}}{c_{1}} \quad \text { und } \quad m_{1}^{*}=\frac{I_{F 1, \text { detekt }}^{\prime}}{c_{1}}
$$

berechnet werden

$$
\beta=\frac{m_{1}}{m_{1}^{*}}=\frac{I_{F 1, \text { detekt }}^{\prime \prime}}{I_{F 1, \text { detekt }}^{\prime}} .
$$

Dabei werden die Proportionalitätsfaktoren $m_{1}$ und $m_{1}^{*}$ ebenfalls durch Minimieren der Fehlerquadratsumme bestimmt. Für die gewählten Einstellungen des Messsystems ergibt sich so ein Verstärkungsfaktor von $\beta=58,9$ und damit ein gutes Signal-Rausch-Verhältnis. Dadurch ist eine sehr sensitive Detektion der chemischen Reaktion möglich.

Die Berechnung der Konzentration des reagierenden Farbstoffs fluo-4 ist nur für die zwei definierten Zustände "vollständig reagiert" oder "vollständig nicht-reagiert" analog zu Gl. (8.13) durchführbar. In allen anderen Fällen setzt sich die gemessene Fluoreszenzintensität

$$
I_{F}^{I}\left(c_{1}\right)=I_{F 1}^{\prime}\left(c_{1, n R}\right)+I_{F 1}^{\prime \prime}\left(c_{1, R}\right)
$$

aus der Fluoreszenzintensität des nicht reagierten Anteils $I_{F 1}^{\prime}\left(c_{1, n R}\right)$ und der Fluoreszenzintensität des bereits vollständig mit Calcium reagierten Anteils $I_{F 1}^{\prime \prime}\left(c_{1, R}\right)$ zusammen. Die in einem Volumenelement insgesamt vorhandene Konzentration des reagierenden Farbstoffes ist also die Summe aus der Konzentration des Reaktionsprodukts $c_{1, R}$ und der Konzentration des Edukts $c_{1, n R}$

$$
c_{1}=c_{1, n R}+c_{1, R}
$$

Aus den Gleichungen (8.15), (8.16) und (8.17) folgt dann

$$
I_{F 1}^{\prime \prime}\left(c_{1, R}\right)=\left[I_{F}^{I}\left(c_{1}\right)-\frac{1}{\beta} I_{F 1}^{\prime \prime}\left(c_{1}\right)\right] \frac{\beta}{\beta-1} .
$$


Aus den oben beschriebenen Kalibriermessungen ist der Proportionalitätsfaktor $m_{1}$ (Gl. (8.14)) für eine homogene Lösung eines vollständig reagierten Systems bekannt. Für diesen Fall gelten die Beziehungen $c_{1}=c_{1, R}$ und $c_{1, R}=\frac{I_{F 1}^{\prime \prime}}{m_{1}}$, so dass für die Konzentration des Reaktionsprodukts gilt

$$
c_{1, R}=\frac{1}{m_{1}}\left[I_{F}^{I}\left(c_{1}\right)-\frac{1}{\beta} m_{1} c_{1}\right] \frac{\beta}{\beta-1} .
$$

Laut Gl. (4.6) lässt sich die Konzentration $c_{1}$ aber auch aus der Konzentration des inerten Farbstoffs $c_{2}$ und dem Anfangskonzentrationsverhältnis $C_{0}=\frac{c_{1,0}}{c_{2,0}}$ berechnen. Berücksichtigt man die Gleichungen (8.13) und (8.11), so ist die Konzentration $c_{1, R}$ ausschließlich aus den Messwerten $I_{F}^{I}$ und $I_{F}^{I I}$ und den Kalibrierfaktoren $f^{I I}, m_{1}, m_{2}$ und $\beta$ berechenbar

$$
c_{1, R}=\frac{1}{m_{1}}\left[I_{F}^{I}-\frac{1}{\beta} \cdot \frac{m_{1}}{m_{2}} \cdot \frac{c_{1,0}}{c_{2,0}}\left(I_{F}^{I I}-f^{I I} I_{F}^{I}\right)\right] \frac{\beta}{\beta-1} .
$$

Die Konzentration des inerten Farbstoffs kann wie oben beschrieben nach Gl. (8.11) und Gl. (8.13) berechnet werden

$$
c_{2}=\frac{I_{F}^{I I}-f^{I I} I_{F}^{I}}{m_{2}}
$$

Beide Konzentrationen werden für jeden Pixel des Kamerachips berechnet. Aus diesen Konzentrationsfeldern der beiden Farbstoffe lässt sich schließlich das Feld des lokalen Deviationsgrades im betrachteten Bildausschnitt mit den Gln. (4.5) und (4.6) berechnen

$$
\Delta=1-\frac{c_{1, R}}{c_{2}} \cdot \frac{c_{2,0}}{c_{1,0}}
$$

\subsection{Ablauf der quantitativen Auswertung}

Aus den gemessenen Intensitäten der Fluoreszenzfarbstoffe werden die Konzentrationsfelder und daraus das Feld des lokalen Deviationsgrades nach dem in Bild 8.14 dargestellten Schema berechnet. Vor Versuchsbeginn sind die in Kapitel 8.2 beschriebenen Kalibrierungen durchzuführen. Zunächst wird ein geeigneter Bildausschnitt gewählt und die Doppelbildoptik eingestellt (s. Kapitel 8.2.1). Dann wird die Intensitätsverteilung einer gering konzentrierten homogenen Farbstofflösung gemessen und daraus die Korrekturmatrix zur Korrektur des Laserprofils berechnet (s. Kapitel 8.2.3). Die Kalibrierfaktoren des Farbstoffsystems werden aus je einer Konzentrationsreihe für jeden Farbstoff bestimmt. Dazu werden die Fluoreszenzintensitäten der homogenen Farbstofflösungen und zeitgleich die Leistung des Lasers (s. Kapitel 8.2.2) gemessen. Ein repräsentativer Mittelwert der Fluoreszenzintensität für eine bestimmte Konzentration wird aus einem zeitlichen Mittel von 200 - 400 Bildern gewonnen. Dieses wird mit der gemessenen Leistung des Lasers und der Korrekturmatrix korrigiert. Schließlich muss der Filterwert $f^{I I}$ bestimmt werden, der angibt, welcher Bruchteil des Fluoreszenzlichts von fluo-4 ungewollt durch den Kantenfilter RG645 für den Farbstoff carboxy-SNARF detektiert wird (s. Kapitel 8.2.4). 


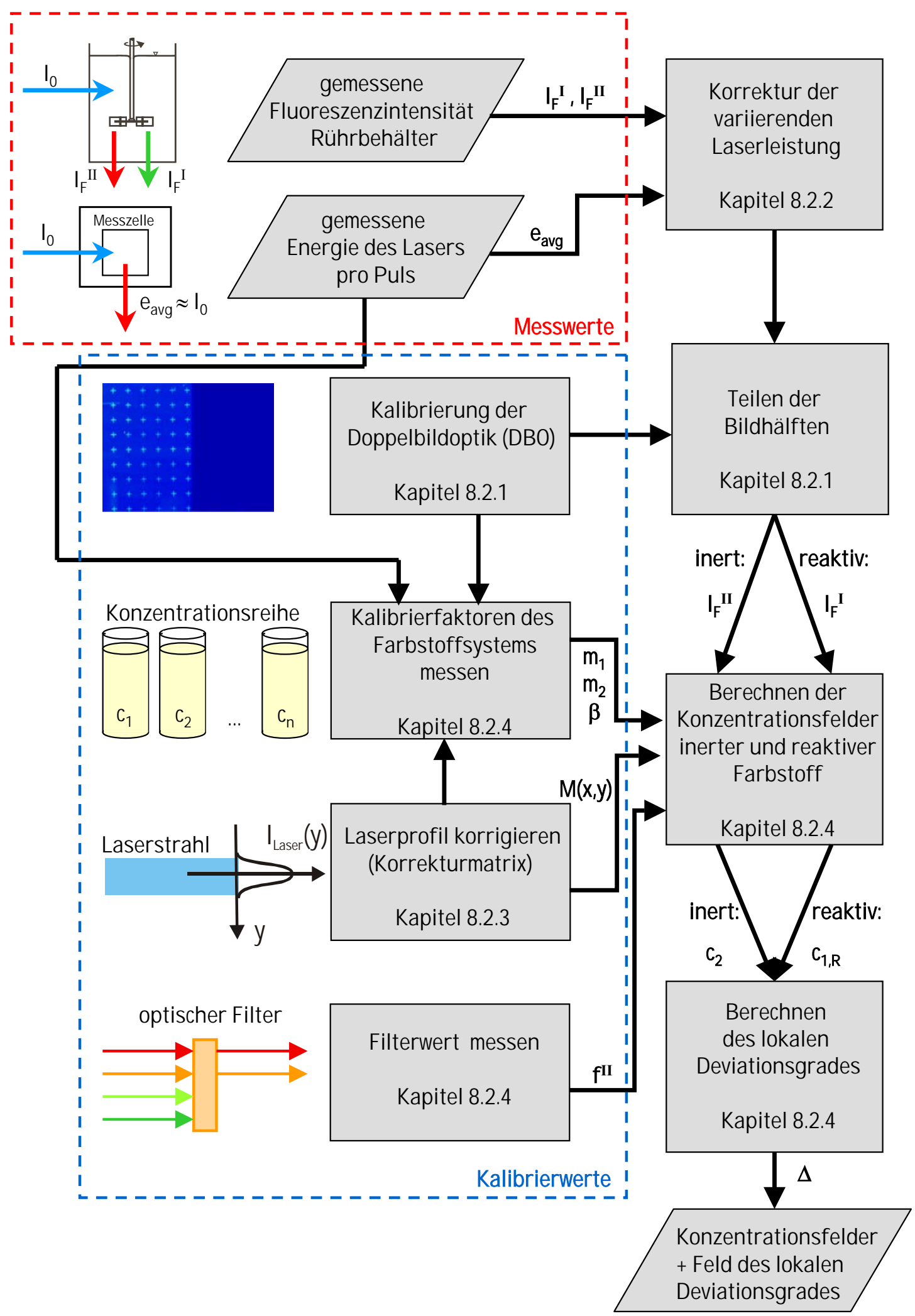

Bild 8.14: Schematischer Ablauf für die quantitative Auswertung der gemessenen Fluoreszenzintensitäten 
Nach Abschluss der Kalibrierungen können Experimente im Rührbehälter durchgeführt werden. Dabei werden sowohl die Fluoreszenzintensitäten der sich im Behälter verteilenden Farbstoffe als auch die Leistung des Lasers gemessen und die Bilder im Computer abgespeichert. Im ersten Schritt der Auswertungen werden alle Bilder entsprechend Kapitel 8.2.2 mit der gemessenen Laserleistung korrigiert. Anschließend erfolgt die Teilung des Bildes, so dass die Felder der Fluoreszenzintensitäten $I_{S}^{I}$ und $I_{S}^{I I}$ in zwei getrennten Bildern vorliegen. Mit Hilfe der gemessenen Kalibrierfaktoren können nun die Konzentrationsfelder des inerten und des reagierenden Farbstoffs wie in Kapitel 8.2.4 beschrieben berechnet werden. Hieraus wird dann in einem abschließenden Schritt das Feld des lokalen Deviationsgrades berechnet. 


\section{Ergebnisse der experimentellen Untersuchungen}

Im ersten Teil dieses Kapitels werden zunächst Betrachtungen zur Messgenauigkeit des Systems durchgeführt. Dazu werden die Konzentrationen von Farbstofflösungen bekannter Konzentration gemessen und so der zu erwartende Messfehler abgeschätzt. Im Weiteren werden die experimentellen Untersuchungen in einem Rührbehälter, der mit einem Sechsblatt-Scheibenrührer ausgestattet ist, erläutert. In den Kapiteln 9.2 und 9.3 werden der Einfluss des Zugabeortes und der Viskosität des zu mischenden Fluides auf das Vermischungsverhalten untersucht. Aus den Messwerten wird in Kapitel 9.4 die Lamellendicke als makroskopischer Längenmaßstab der Segregation ermittelt. Die Abhängigkeit von den oben genannten Einflussparametern wird schließlich erläutert und physikalisch interpretiert.

\subsection{Messgenauigkeit}

In Kapitel 8.2.4 wurde bereits Kalibrierungsprozess des Messsystems erläutert. Aus Messwerten der Fluoreszenzintensität $I_{F}$ für Farbstofflösungen bekannter Konzentration $c$ wird durch Regression der Proportionalitätsfaktor $m$ der linearen Gleichung $I_{F}=m c$ ermittelt. Mit Hilfe dieses Ansatztes kann anschließend aus gemessenen Fluoreszenzintensitäten die Konzentration berechnet werden. Für diese Kalibriermessungen werden Farbstofflösungen mit jeweils nur einem Farbstoff verwendet. Die Messgenauigkeit bei Vorliegen eines Farbstoffgemisches wird im Folgenden abgeschätzt.

Dazu werden Farbstofflösungen hergestellt, die jeweils eine bekannte Konzentration an carboxy-SNARF und fluo-4 enthalten, wobei Konzentrationsverhältnisse zwischen 0,4 und 10 gewählt werden. Wie schon für die Kalibrierung wird aus Kostengründen nur ein Volumen von $30 \mathrm{ml}$ pro Farbstoffgemisch hergestellt. Die Konzentrationen $8 \cdot 10^{-8} \mathrm{~mol} / \mathrm{l}$ und $2 \cdot 10^{-7} \mathrm{~mol} / \mathrm{l}$ werden in mehreren Lösungen mit unterschiedlichen Konzentrationsverhältnissen eingestellt, alle anderen Konzentrationen werden nur in einer Lösung verwendet. Für jedes Farbstoffgemisch werden die Messwerte zeitlich über 300 Bilder gemittelt und die Messungen mehrmals (2-4 mal) wiederholt. Anschließend wird jeweils ein örtlicher Mittelwert über ein repräsentatives Gebiet in dem Glasbehälter ermittelt. Aus den gemessenen Fluoreszenzintensitäten werden gemäß dem in Bild 8.14 erläuterten Ablauf die Konzentrationen der beiden Farbstoffe berechnet. 


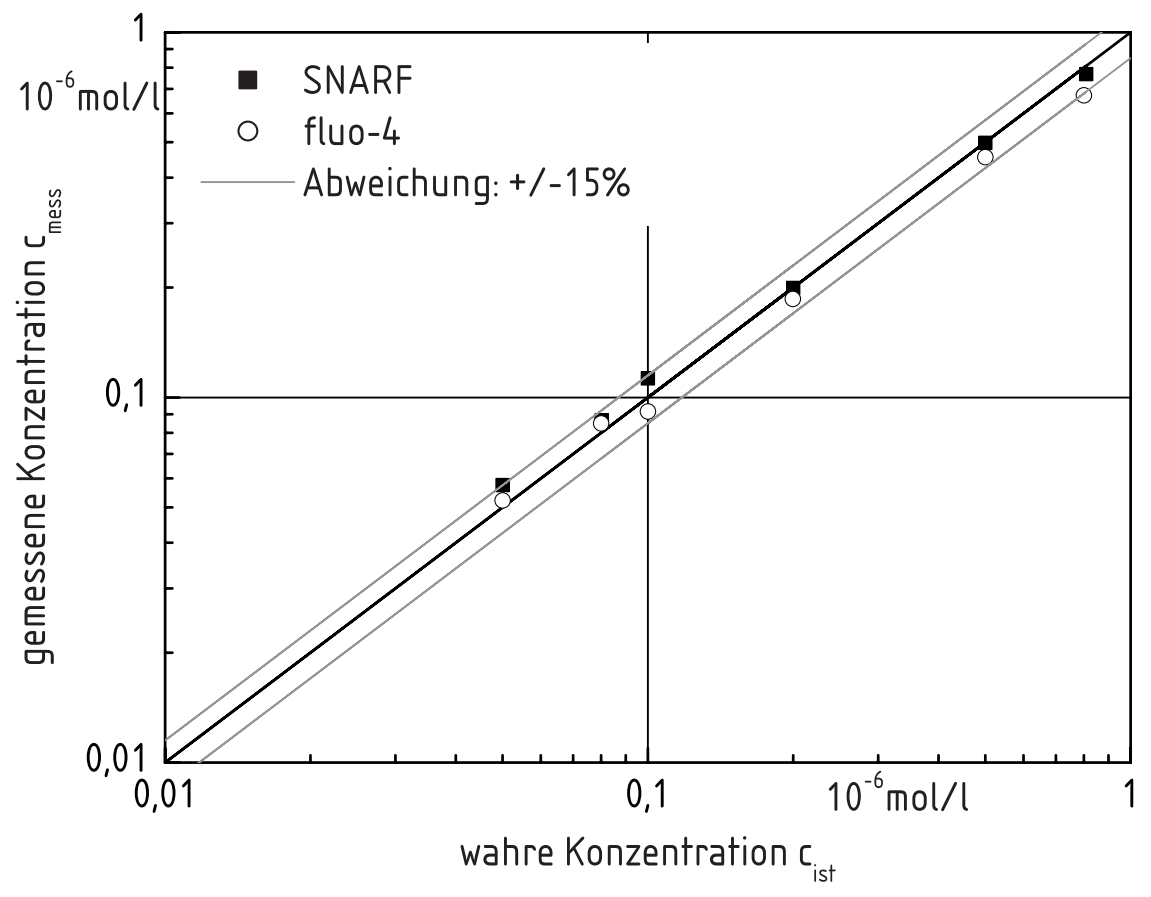

Bild 9.1: Gemessene Konzentration $c_{m e s s}$ als Funktion der wahren Konzentration $c_{i s t}$

In Bild 9.1 ist die gemessene Konzentration über der wahren Konzentration aufgetragen. Die maximale Abweichung beträgt sowohl für carboxy-SNARF als auch für fluo-4 15\%. Gleichzeitig wird der Fehler bei der Bestimmung der wahren Konzentration zu etwa 3\% bis 9\% abgeschätzt, was sich aus Ungenauigkeiten beim Pipettieren der hochkonzentrierten Farbstofflösungen ergibt. Die relative Standardabweichung der einzelnen Messwerte von dem dargestellten Mittelwert beträgt für carboxy-SNARF weniger als 0,8\%. Die einzige Ausnahme bildet die Konzentration $8 \cdot 10^{-8} \mathrm{~mol} / \mathrm{l}$ mit einer relativen Standardabweichung von 3\%. Dabei liegen sechs Messwerte von zwei unterschiedlichen Farbstoffgemischen zugrunde. Für den reagierenden Farbstoff fluo-4 beträgt die relative Standardabweichung weniger als 0,3\% für die Konzentrationen, die nur in einer Farbstoffösung gemessen wurden. Für die in mehreren Farbstofflösungen gemessenen Konzentrationen ergibt sich eine relative Standardabweichung von $2 \%$ bis $7 \%$.

\subsection{Einfluss des Zugabeortes}

Die Versuchsflüssigkeiten werden wie in Kapitel 6.3 beschrieben vorbereitet. Die Anfangskonzentrationen der Farbstoffe betragen $c_{2,0}=2,2 \cdot 10^{-6} \mathrm{~mol} / \mathrm{l}$ für den inerten Farbstoff carboxy-SNARF und $c_{1,0}=1,02 \cdot 10^{-6} \mathrm{~mol} / \mathrm{l}$ für den reaktiven Farbstoff fluo-4. Alle Versuchsflüssigkeiten enthalten gelöste Zellulose mit der Massenkonzentration 1\%. Der Rührer ist mit dem Bodenabstand von $50 \mathrm{~mm}$ in den Behälter eingebaut. Die Rührer- 


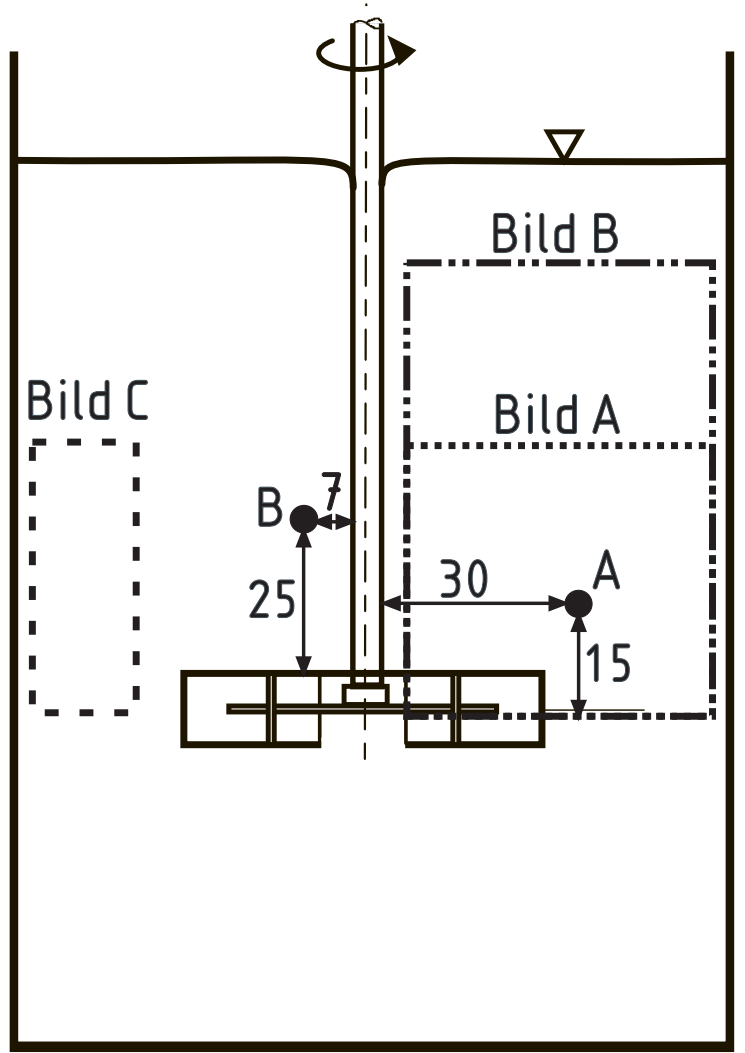

Bild 9.2: Position der Farbstoffzugabe im Rührbehälter

drehzahl von $n_{r}=250 \mathrm{~min}^{-1}$ resultiert näherungsweise in einer Reynoldszahl von $R e \approx 42$. Die Vermischung findet somit im Wesentlichen im laminaren Strömungsbereich statt. Das Farbstoffgemisch wird an den in Bild 9.2 dargestellten Positionen 'A' und 'B' in den Rührbehälter dosiert. Position 'A' ist nahe dem Zentrum des oberen Ringwirbels, während sich Position 'B' näher an der Rührerwelle befindet. Die Zugabeorte unterscheiden sich sehr stark in den lokalen Strömungsgeschwindigkeiten. In Bild 7.6 ist das aus einer Simulation bestimmte stationäre Strömungsfeld im Rührbehälter mit Hilfe von Vektoren in der Symmetrieebene des Behälters dargestellt. Für die Position im Ringwirbelzentrum ist die radiale und axiale Geschwindigkeitskomponente nahezu Null, und es erfolgt überwiegend ein konvektiver Transport in Umfangsrichtung. An der Position 'B' nahe der Rührerwelle hat die Strömungsgeschwindigkeit hingegen eine große Komponente in axialer Richtung zur Rührerebene hin. Ein dort eindosierter Farbstofftropfen wird schneller in Bereiche hoher Deformationsgeschwindigkeiten transportiert. Diese sind vor allem in der Rührerebene nahe den Rührerblätter zu finden (s. Bild 7.6). Für den Zugabeort 'B' wird deshalb eine ausgeprägtere Scherung des eindosierten Farbstofftropfens erwartet.

In Bild 9.2 ist der Bildausschnitt der Aufnahme schematisch durch den mit 'Bild B' markierten Rahmen dargestellt. Er befindet sich in der Symmetrieebene des Behälters, während die Zugabeorte um etwa $90^{\circ}$ versetzt dazu sind. Der Bildausschnitt hat eine Größe von 35 mm x 62 mm, so dass ein Pixel des Kamerachips etwa 0,13 mm entspricht. 
In Bild 9.3 sind die Ergebnisse bei Zugabe des Farbstoffs in der Nähe des Zentrums des oberen Ringwirbels (Position 'A') dargestellt. Der Bildausschnitt während der Aufnahme entspricht, wie oben beschrieben, dem mit 'Bild B' markierten Rahmen. Da aber in weiten Teilen des Bildes keine Vermischung zu beobachten ist, ist in Bild 9.3 nur der kleinere Bildausschnitt 'Bild A' dargestellt. Seine Größe beträgt etwa 35 mm x 35 mm. In der oberen Bilderreihe sind die Konzentrationsfelder des inerten Farbstoffs und in der unteren Reihe die aus den gemessenen Konzentrationen berechneten Felder des lokalen Deviationsgrades dargestellt. Nach einer Mischdauer von $4 \mathrm{~s}$ ist der Tropfen fast ausschließlich eindimensional in Umfangsrichtung gedehnt. Der Längenmaßstab der Segregation ist noch sehr groß, so dass nur ein langsamer diffusiver Stofftransport stattfinden kann. Somit nimmt der lokale Deviationsgrad in weiten Teilen sehr große Werte nahe Eins an. Nach $11 \mathrm{~s}$ ist eine Verformung der Lamelle entlang des Ringwirbels in radialer und axialer Richtung und die Bildung mehrerer Lamellen zu erkennen. Überwiegend in der Grenzfläche der Lamelle ist der Wert des lokalen Deviationsgrades bereits gesunken, was auf den lokalen Fortschritt der chemischen Reaktion und damit der Mikromischung hinweist. Auch $29 \mathrm{~s}$ nach der Zugabe ist noch keine prinzipielle Änderung der Vermischungsstruktur erkennbar. Die Lamellen bilden mehrschichtige Strukturen, bleiben aber ausschließlich nahe dem

Makromischen: Konzentration $c_{2}$
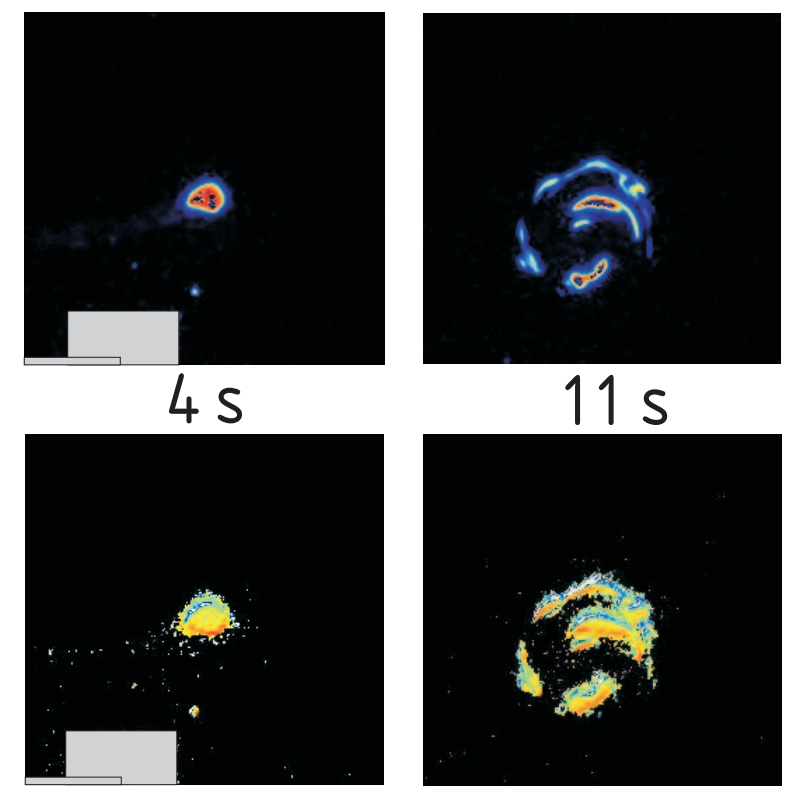

Mikromischen:

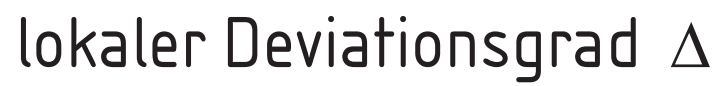

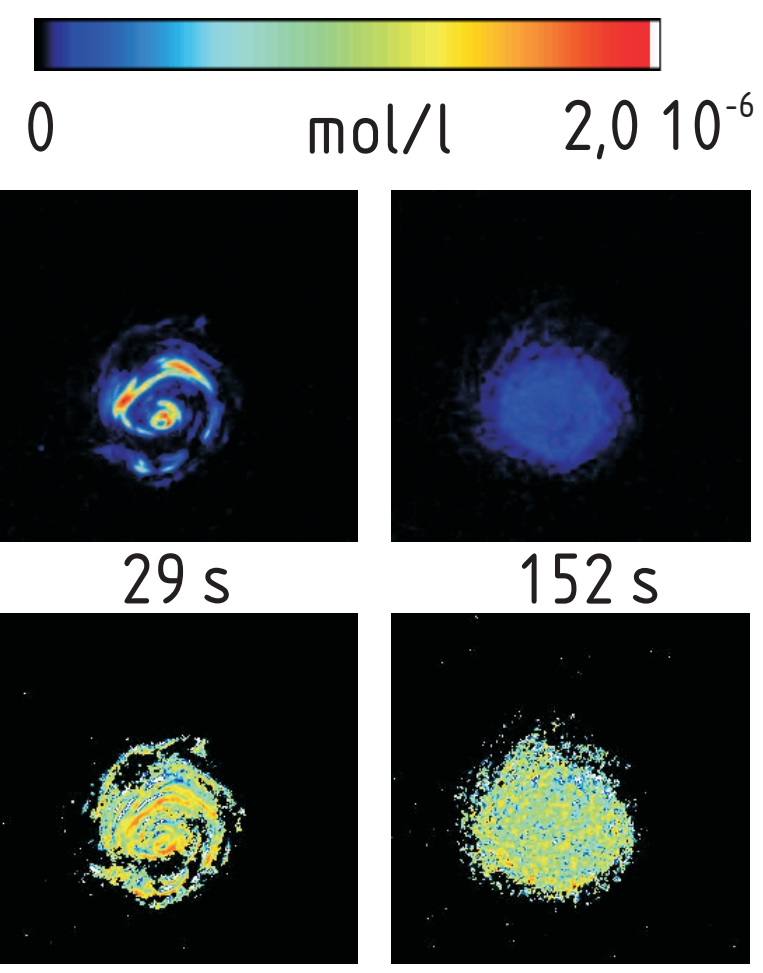

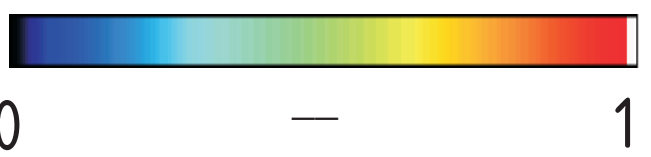

Bild 9.3: Ablauf des Makro- und Mikromischens für die Zugabeposition 'A' 
Zentrum des Ringwirbels. Erst nach 152 s sind die Lamellen so dünn, dass ihre Struktur nicht mehr aufgelöst werden kann und nur noch eine Farbstoffwolke erkennbar ist. Die Größe der Vermischungszone weicht jedoch nicht wesentlich von derjenigen nach $7 \mathrm{~s}$ ab. In dieser Zone ist der lokale Deviationsgrad auf einen nahezu konstanten Wert von etwa 0,3 abgefallen, was bedeutet, dass der Farbstoff noch immer nicht vollständig mit seiner Umgebung mikrovermischt ist.

Dagegen ist wie erwartet für die Zugabeposition 'B' (nahe der Rührerwelle) eine deutlich effizientere Makro- und Mikrovermischung zu beobachten. In der oberen Reihe von Bild 9.4 sind wieder die Konzentrationsfelder des inerten Farbstoffs und in der unteren Reihe die berechneten Felder des lokalen Deviationsgrades dargestellt. Bereits nach 2 s hat sich eine periodisch wiederkehrende Lamellenstruktur ausgebildet. Neue Schichten werden jeweils erzeugt, wenn sich ein Rührerblatt durch die Messebene bewegt. Die Lamellen werden zur Wand hin transportiert, dort umgelenkt, weiter gestreckt und gefaltet und erneut durch die Rührerebene transportiert. Die Abmessungen der entstandenen Schichtenstruktur, die Lamellendicke, ist deutlich geringer als bei Zugabe nahe dem Ringwirbelzentrum. In Kapitel 9.4 wird die Bemaßung der Lamellendicke näher erläutert und diese für unterschiedliche Vermischungen quantifiziert.

Tendenziell ist zu erkennen, dass der lokale Deviationsgrad im Zentrum der Lamellen hohe Werte annimmt, während er in den Grenzflächen bereits abgenommen hat. Letzteres weist auf den lokalen Fortschritt der chemischen Reaktion und damit der Mikrovermischung hin. Nach 15 s kann die Schichtenstruktur der Lamellen nicht mehr aufgelöst werden und es ist nur noch eine Farbstoffwolke zu erkennen. Die Vermischungszone ist allerdings größer als diejenige für die Zugabeposition 'A'. Der lokale Deviationsgrad ist auf Werte von etwa 0,3 bis 0,4 abgefallen, was bedeutet, dass der Farbstoff noch nicht vollständig mit seiner Umgebung mikrovermischt ist.

Die Lamellenschichten sind noch deutlicher zu erkennen, wenn ein kleinerer Bildausschnitt vergrößert aufgenommen wird. In Bild 9.5 wird nur der wandnahe Bereich abgebildet, so dass 1 Pixel etwa 0,07 mm entspricht. Die genaue Position des $14 \mathrm{~mm}$ x $32 \mathrm{~mm}$ großen Bildausschnitts ist in Bild 9.2 mit 'Bild C' gekennzeichnet. Das Stauchen der Farbstoffschichten durch die Umlenkung an der Behälterwand ist zu erkennen. In den ersten $3 \mathrm{~s}$ wird erneut die oben erwähnte Tendenz beobachtet, dass der lokale Deviationsgrad im Zentrum der Lamelle höhere Werte annimmt als in den Randbereichen. Die Schichtenstruktur in Wandnähe ist auch nach $7 \mathrm{~s}$ noch zu erkennen, während die örtliche Auflösung in Bild 9.4 dafür nicht ausreichend ist. Die Vermischung in diesem Bereich hoher Scherung ist nach $7 \mathrm{~s}$ bereits weit fort geschritten. Der lokale Deviationsgrad hat überwiegend Werte um 0,4 bis 0,6 angenommen. 
Makromischen:

Konzentration $c_{2}$
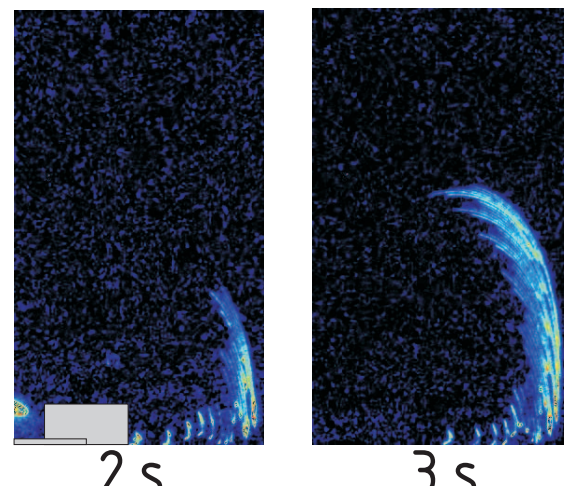

$3 s$
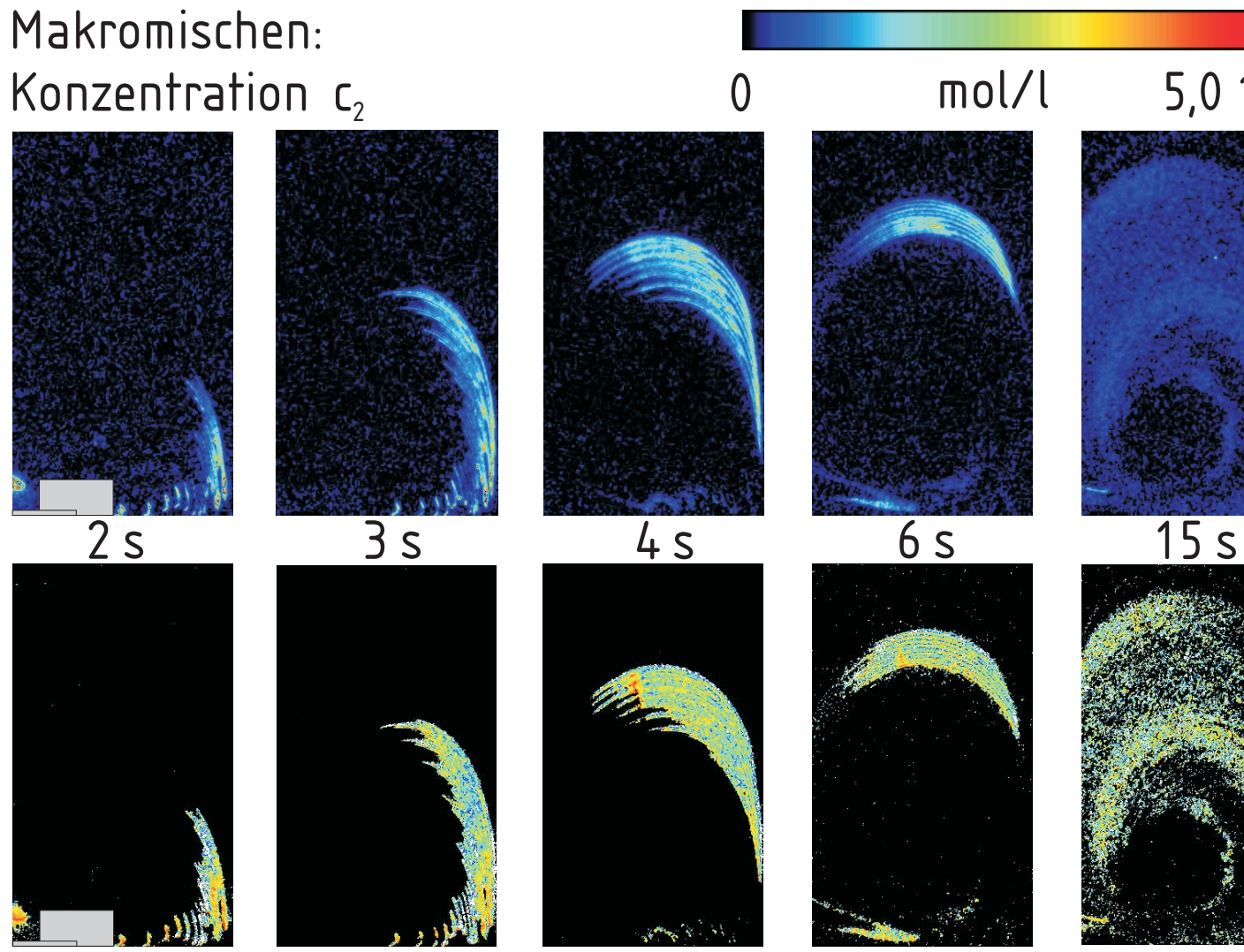

Mikromischen:

lokaler Deviationsgrad $\Delta$

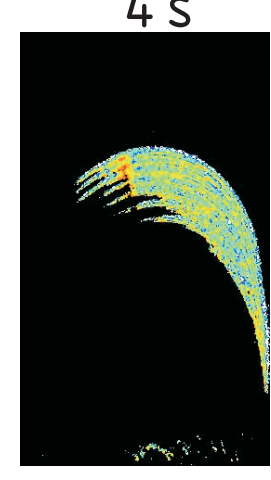

Bild 9.4: Ablauf des Makro- und Mikromischens für die Zugabeposition 'B'
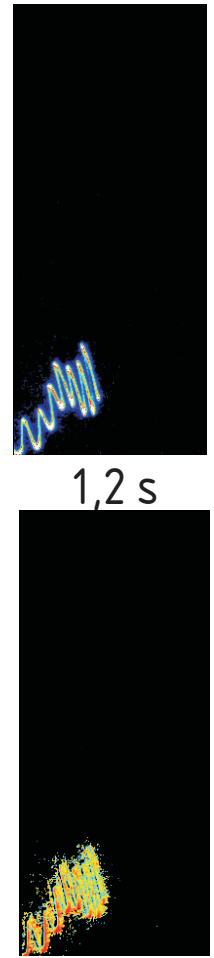
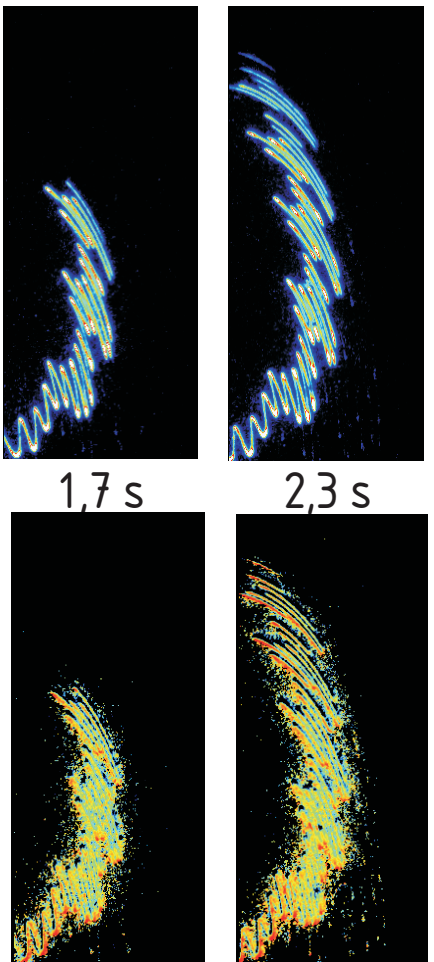

$2,3 \mathrm{~s}$
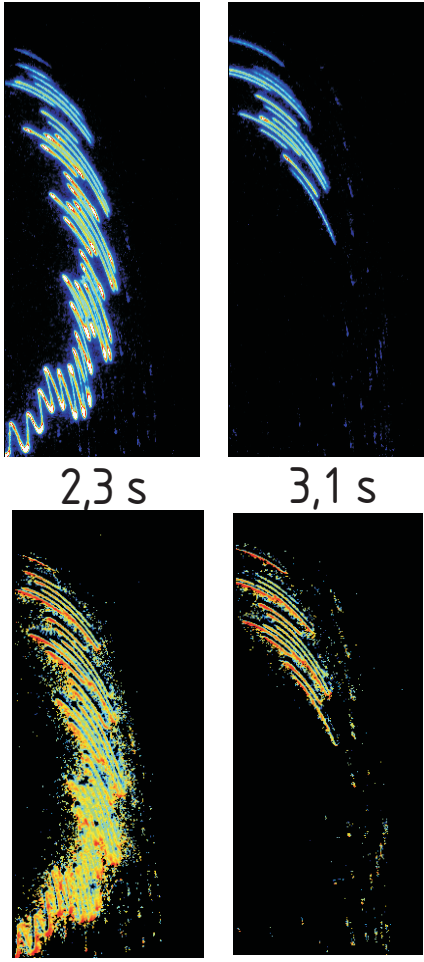

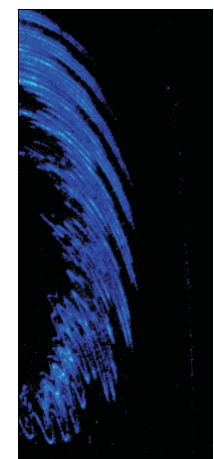

$7,0 \mathrm{~s}$
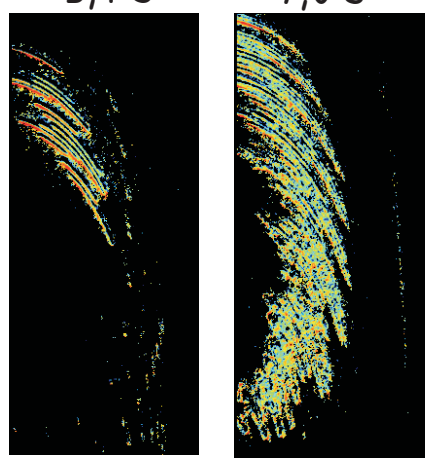

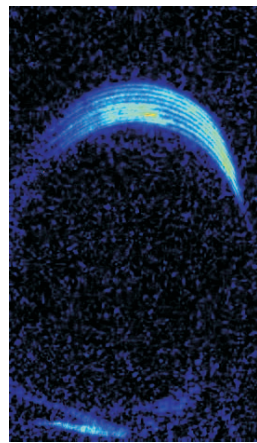

$6 s$

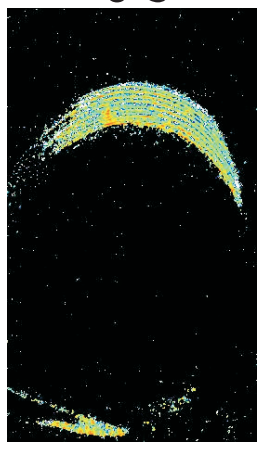

$15 s$
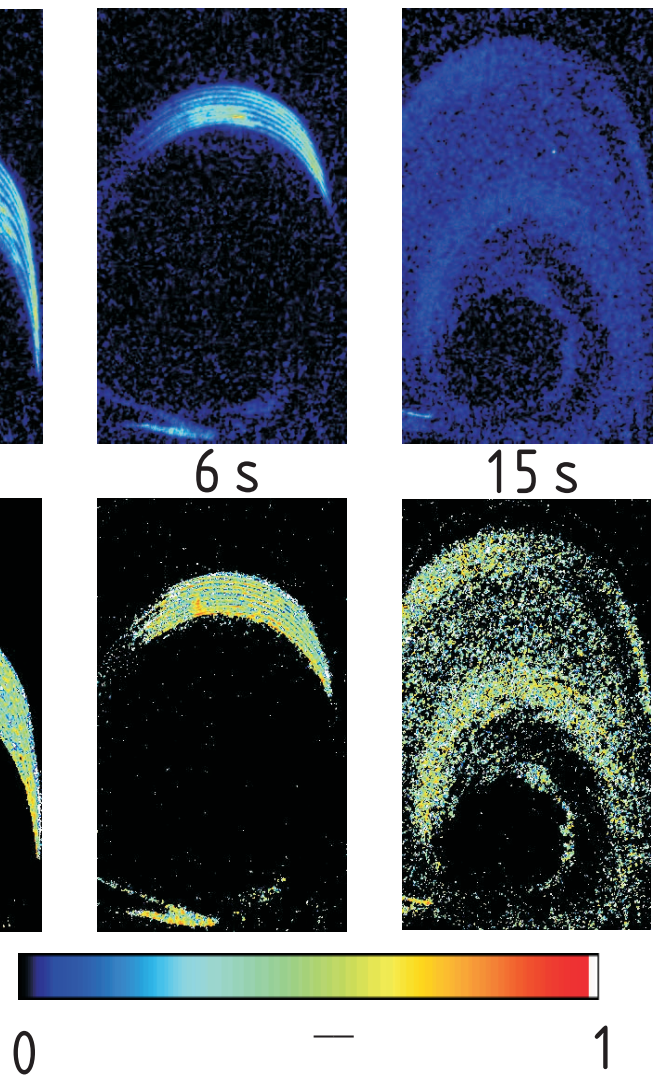

$5,010^{-7}$

Makromischen: Konzentration $c_{2}$

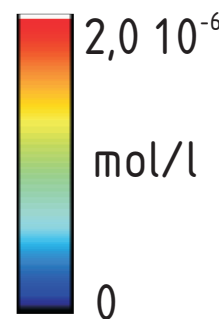

Mikromischen:

lokaler

Deviationsgrad $\Delta$

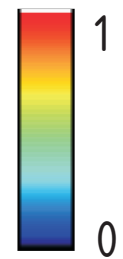

Bild 9.5: Ablauf des Makro- und Mikromischens für die Zugabeposition 'B'- Bildausschnitt 'C' 


\subsection{Einfluss der Viskosität}

Die Viskosität der zu vermischenden Fluide hat einen großen Einfluss auf das Strömungsfeld im Rührbehälter und damit auf die Vermischung. In Kapitel 7.5 wurden berechnete Ergebnisse zu Geschwindigkeits- und Viskositätsfeldern für drei Zelluloselösungen mit unterschiedlichen Massenanteilen $\xi_{1}=0,5 \%, \xi_{2}=1,0 \%$ und $\xi_{3}=2,0 \%$ vorgestellt. Vergleichbare Strömungsfelder werden bei gleicher Reynoldszahl (s. Gl. 7.26) erwartet. Die Reynoldszahl ist vom Durchmesser des Rührorgans $d_{r}$, seiner Drehzahl $n_{r}$ sowie von den Stoffparametern Dichte $\rho$ und Viskosität $\eta$ abhängig. Letztere ist, wie oben beschrieben, für strukturviskose Fluide eine Funktion der Schergeschwindigkeit $\dot{\gamma}$ und damit im Rührbehälter nicht konstant. Aufgrund der örtlich veränderlichen Viskosität ist es nicht möglich, eine für den gesamten Rührbehälter gültige Reynoldszahl zu berechnen. Dennoch ist es wünschenswert, mit Hilfe der Reynoldszahl annähernd eine Einordnung in den laminaren oder turbulenten Strömungsbereich machen zu können. Für $R e<10$ (für randgängige Rührer $R e<100)$ liegt erfahrungsgemäß eine laminare Strömung vor [25]. Es schließt sich ein relativ großer Übergangbereich an, und bei $R e>10^{4}$ kann von einer nahezu isotropen Turbulenz im Rührbehälter ausgegangen werden. Mit Hilfe eines empirischen Verfahrens nach Metzner und Otto [25] kann aus einer repräsentativen Viskosität $\eta_{\text {rep }}$ die Reynoldszahl nach Gl. (7.26) berechnet werden.

Voraussetzung ist, dass aus rheologischen Messungen die Fließkurve $\eta=f(\dot{\gamma})$, wie in

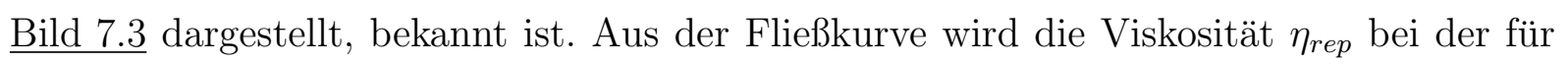
den Prozess repräsentativen Schergeschwindigkeit $\dot{\gamma}_{r e p}=\dot{\gamma}$ abgelesen, die sich aus der Drehfrequenz des Rührorgans $n_{r}$ berechnen lässt

$$
\dot{\gamma}_{r e p}=k_{M / O} \cdot n_{r}
$$

Die Metzner-Otto Konstante $k_{M / O}$ ist für einige Rührertypen bekannt und gilt jeweils für geometrisch ähnliche Rührer. Für einen Sechsblatt-Scheibenrührer und ein strukturviskoses Fluid gilt $k_{M / O}=12$ [25].

Ist eine Reynoldszahl vorgegeben, so ist die zugehörige Drehzahl aus den Gleichungen (7.26) und (9.1) iterativ zu bestimmen. Für eine konstante Reynoldszahl nimmt die benötigte Drehfrequenz mit steigender Viskosität der verwendeten Zelluloselösungen zu. Um eine Reynoldszahl von $R e=40 \mathrm{zu}$ erreichen, wird eine Rührerdrehzahl von $n_{r 1}=60 \mathrm{~min}^{-1}$ für die Zelluloselösung mit $\xi_{1}=0,5 \%, n_{r 2}=250 \mathrm{~min}^{-1}$ für die Zelluloselösung mit $\xi_{2}=1,0 \%$ und eine Drehzahl von $n_{r 3}=750 \mathrm{~min}^{-1}$ für die Zelluloselösung mit $\xi_{3}=2,0 \%$ benötigt.

In diesem Kapitel werden nur die Zelluloselösungen mit $\xi_{1}=0,5 \%$ und $\xi_{3}=2,0 \%$ betrachtet, da die Ergebnisse für $\xi_{2}=1,0 \%$ in Kapitel 9.2 bereits ausführlich erläutert 
wurden. Die Farbstoffzugabe erfolgt jeweils am Zugabeort 'B' nahe der Rührerwelle.

In Bild 9.6 ist der Ablauf des Makro- und Mikromischens für die niedrigviskose Zelluloselösung mit $\xi_{1}=0,5 \%$ dargestellt. In der oberen Reihe sind die Konzentrationsfelder des inerten Farbstoffs und in der unteren Reihe die berechneten Felder des lokalen Deviationsgrades dargestellt. Es fällt auf, dass sowohl die Lamellenstruktur als auch die Zeitskala der Vermischung sehr ähnlich zu den in Kapitel 9.2 gezeigten Ergebnissen für die Zelluloselösung mit $\xi_{2}=1,0 \%$ sind. Jedoch ist zu Beginn der Vermischung der Abstand zwischen neu gebildeten Lamellen in der Rührerebene für die niedrigviskose Lösung größer. Dies erklärt sich aus der geringeren Drehzahl, wodurch der Farbstofftropfen in Umfangsrichtung langsamer transportiert wird. Nach $14 \mathrm{~s}$ ist die Vermischung schon sehr weit fortgeschritten, lediglich im oberen Teil des Ringwirbels ist noch ein Bereich höherer Konzentration zu erkennen. Der lokale Deviationsgrad ist allerdings auch dort schon auf Werte zwischen 0,3 und 0,5 gesunken.

Makromischen:

Konzentration $c_{2}$

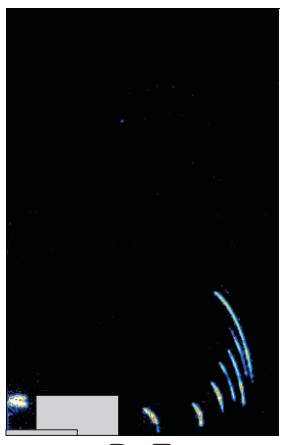

$3,5 \mathrm{~s}$

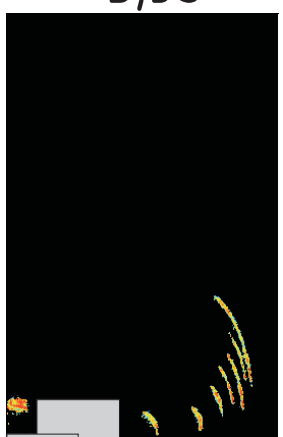

Mikromischen:

lokaler Deviationsgrad $\Delta$

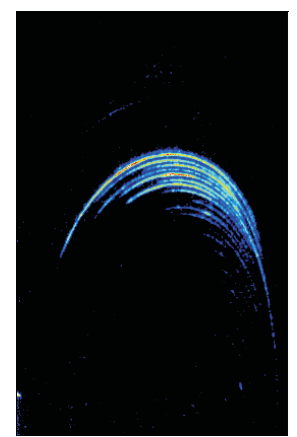

$5,9 \mathrm{~s}$

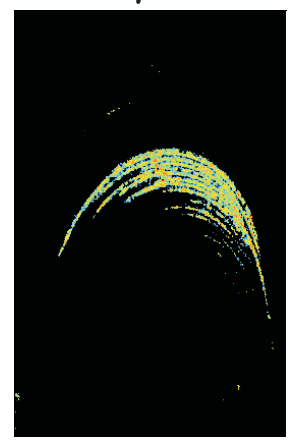

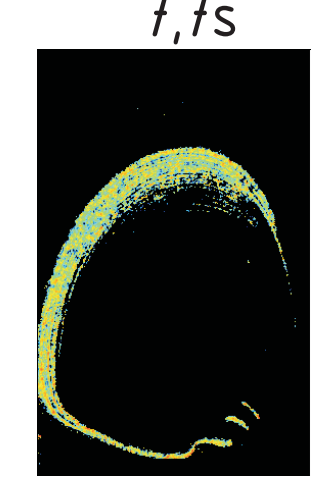

0

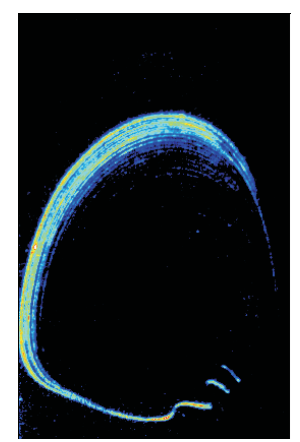

$7,7 \mathrm{~s}$

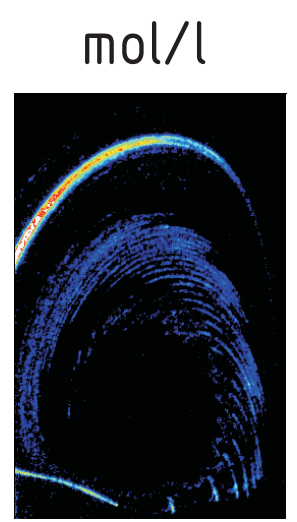

$12,2 s$

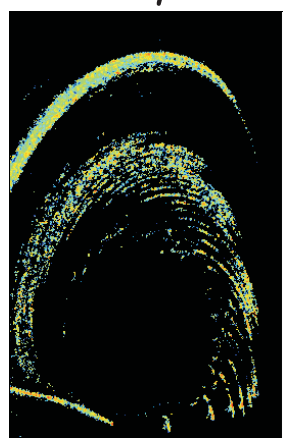

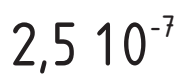

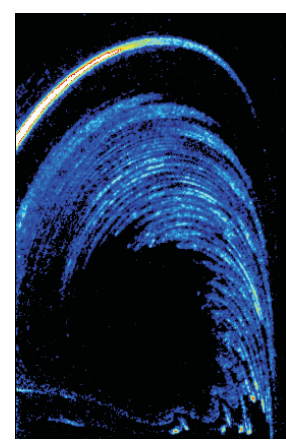

$14,0 \mathrm{~s}$

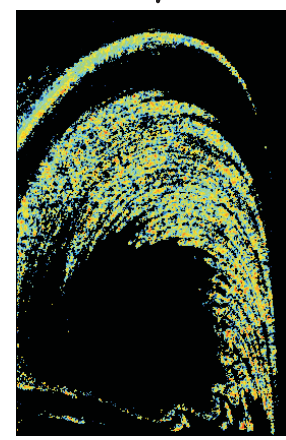

0

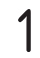

Bild 9.6: Ablauf des Makro- und Mikromischens für die Zelluloselösung mit $\xi_{1}=0,5 \%$ und Zugabeposition 'B' 
Ganz anders sieht die Vermischungsstruktur für die höherviskose wässrige Zelluloselösung mit $\xi_{3}=2,0 \%$ aus. In der oberen Reihe von Bild 9.7 sind die Konzentrationsfelder des inerten Farbstoffs und in der unteren Reihe die berechneten Felder des lokalen Deviationsgrades dargestellt. Es fällt auf, dass der Farbstoff trotz der hohen Drehzahl von $n_{r 3}=750 \mathrm{~min}^{-1}$ kaum in die Rührerebene transportiert wird, wo durch Falten neue Lamellen entstehen könnten. Statt dessen sind auch nach 21 s nur drei Lamellen mit großer Lamellendicke im oberen Teil des Behälters zu sehen. Der lokale Deviationsgrad ist dabei sehr unterschiedlich: In zwei Lamellen ist er schon stark abgesunken, während er in der dritten Lamelle noch sehr hohe Werte bis 0,9 annimmt. Erst nach 48s hat sich eine ausgeprägtere Lamellenstruktur mit dünneren Lamellen ausgebildet. Weiterhin sind bereits Bereiche mit kleinen Werten für den lokalen Deviationsgrad, aber auch noch ausgeprägte Zonen mit hohen Werten nahe Eins zu erkennen. Der Bereich der Kaverne um die

Makromischen:

Konzentration $c_{2}$

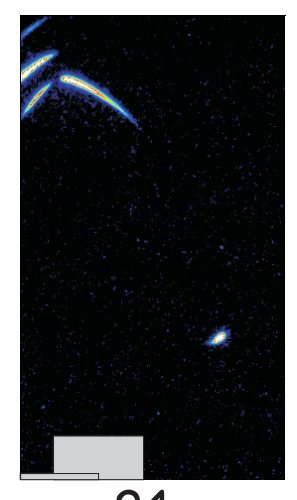

$21 \mathrm{~s}$
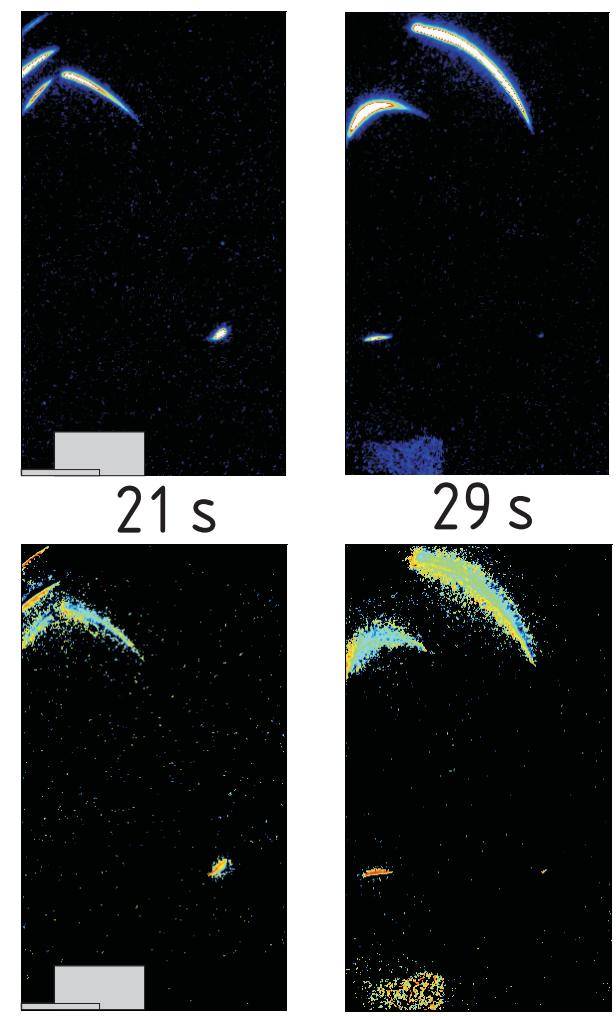

$29 \mathrm{~s}$

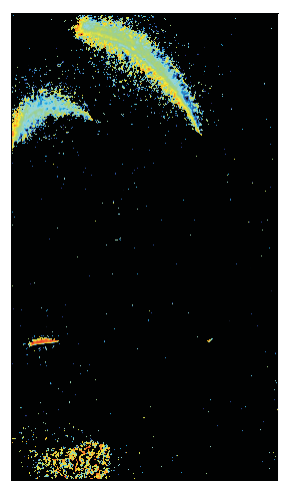

Mikromischen: lokaler Deviationsgrad $\Delta$
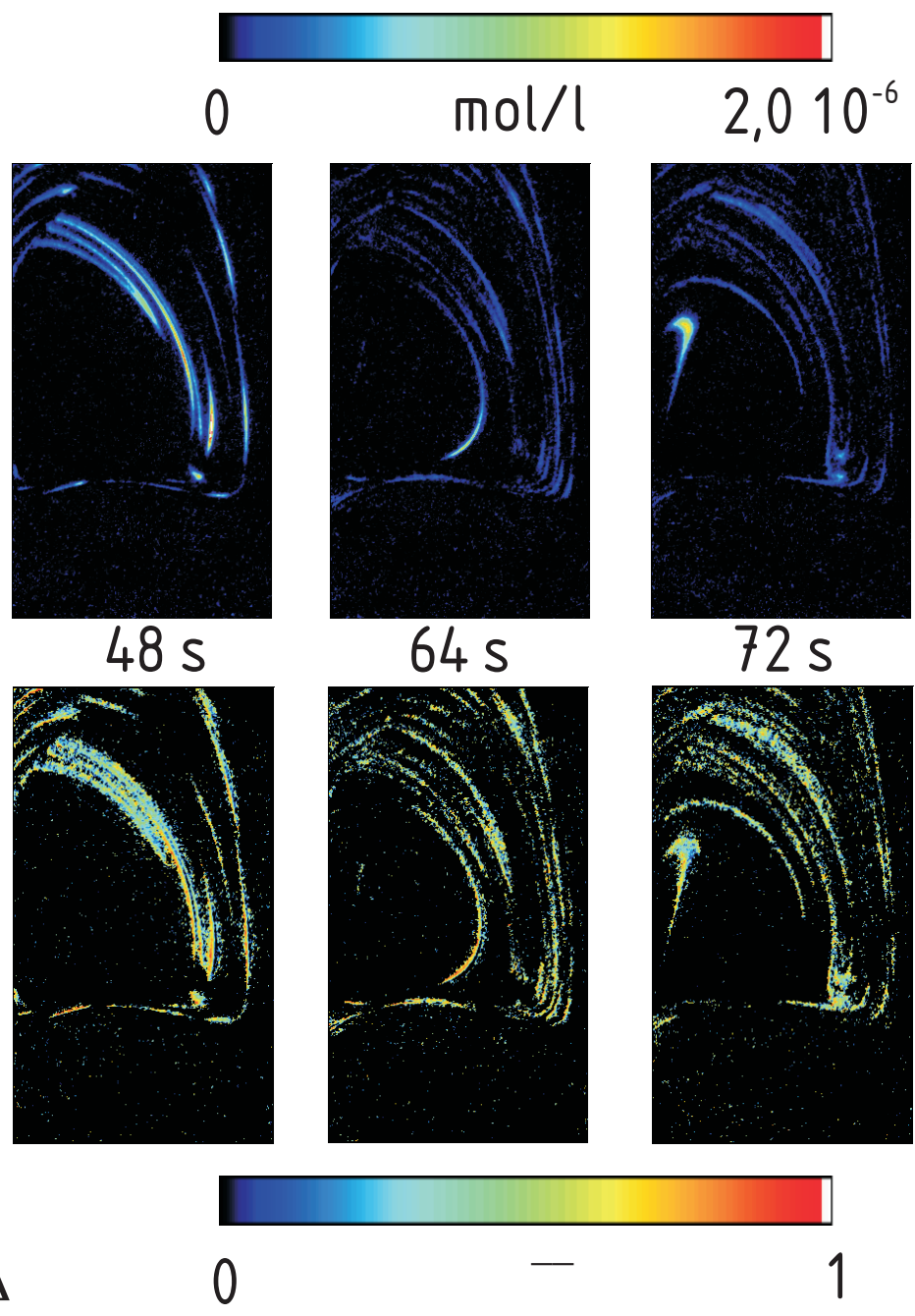

Bild 9.7: Ablauf des Makro- und Mikromischens für die Zelluloselösung mit $\xi_{2}=2,0 \%$ und Zugabeposition 'B' 
Rührerebene (s. Bild 7.9), in der aufgrund der erhöhten Deformationsgeschwindigkeiten eine effektivere Vermischung möglich wäre, bleibt aber frei von Farbstoff. Dies ändert sich auch nach 72 s nicht. Der lokale Deviationsgrad hat aber inzwischen einen nahezu konstanten Wert von etwa 0,2 bis 0,3 angenommen. Trotz sehr schlechter Makrovermischung ist die Mikrovermischung also bereits weit fortgeschritten.

Zusammenfassend kann man sagen, dass mit zunehmender Viskosität sowohl die Zeitskala der Vermischung als auch der Längenmaßstab der Segregation zunimmt.

\subsection{Lamellendicke}

Wie in den Kapiteln 9.2 und 9.3 erläutert, entstehen während der Vermischung im laminaren Strömungsbereich lamellenartige Strukturen, wobei sich jeweils eine Schicht mit Farbstoff und eine Schicht ohne Farbstoff abwechseln. Je nach Viskosität der Lösung und der Strömungsgeschwindigkeit im Zugabeort entstehen unterschiedliche Lamellenstrukturen. Der Abstand der Schichtungen, die Lamellendicke, ist die Längenskala der Segregation (s. Kapitel 4). Je kleiner sie ist, desto schneller können die Konzentrationsunterschiede über die Schichtungen durch diffusiven Stofftransport ausgeglichen werden. Die Lamellendicke ist vom Ort im Rührbehälter und der Zeit nach der Farbstoffzugabe abhängig. Um die globale Mischgüte in Abhängigkeit makroskopischer Parameter, wie z.B. geometrischen Abmessungen oder Stoffgrößen, abschätzen zu können, wird eine gemittelte Lamellendicke betrachtet. Im Folgenden wird die Bemaßung der Lamellen erläutert und der Einfluss der Viskosität, der Rührerdrehzahl und des Zugabeortes auf die Lamellendicke physikalisch interpretiert.

Für jeweils einen Versuch, in dem die Parameter Viskosität, Rührerdrehzahl und Zugabeort konstant sind, wird eine gemittelte Lamellendicke bestimmt. Dazu wird zu unterschiedlichen Zeitpunkten an mehreren Stellen die Lamellendicke gemessen, und daraus das arithmetische Mittel gebildet. In Bild 9.8 ist auf der linken Seite beispielhaft das Konzentrationsfeld des inerten Farbstoffs etwa 4 s nach der Zugabe in die Zelluloselösung mit $\xi_{2}=1,0 \%$ dargestellt. Die Rührerdrehzahl beträgt $300 \mathrm{~min}^{-1}$, was einer Re-Zahl von etwa 52 entspricht. Im oberen Teil des Bildes ist deutlich die Lamellenstruktur, also die Schichtenbildung aus Zelluloselösung mit Farbstoff und solcher die nahezu frei davon ist, zu erkennen. Im rechten Teil des Bildes ist die Konzentration $c_{2}$ über der Bildhöhe $y$ dargestellt, was einem vertikalen Schnitt durch das Konzentrationsfeld bei der horizontalen Koordinate $x=20,97 \mathrm{~mm}$ entspricht. Die mit Farbstoff belegten Lamellen sind als Peaks hoher Konzentration $\left(c_{2} \approx 25-40 \cdot 10^{-8} \mathrm{~mol} / \mathrm{l}\right) \mathrm{zu}$ erkennen. Allerdings sind auch die Schichten dazwischen aufgrund von Diffusion nicht vollständig frei von Farbstoff. Hier beträgt die Konzentration immer noch etwa $c_{2} \approx 10^{-7} \mathrm{~mol} / \mathrm{l}$. Um die Schichtdicke 
bestimmen zu können, muss also ein geeigneter Filterwert gewählt werden. In Bild 9.8 ist dies $c_{\text {Filter }}=11,5 \cdot 10^{-8} \mathrm{~mol} / \mathrm{l}$, so dass für $c_{2}>c_{\text {Filter }}$ eine Schicht mit Farbstoff und für $c_{2}<c_{\text {Filter }}$ eine Schicht ohne Farbstoff definiert ist. Auf diese Weise können fünf der insgesamt sieben Konzentrationspeaks als Lamellen bemaßt werden.

Da die Konzentrationen mit dem Fortschritt der Vermischung und damit der Verdünnung variieren, ist es schwer, einen allgemein gültigen Filterwert für die Bemaßung der Lamellendicke zu ermitteln. Deshalb liegt es nahe, den lokalen Deviationsgrad zu betrachten. Große Werte des Deviationsgrads bedeuten ein hohes Maß an Segregation, während die Mikromischung mit kleiner werdenden Werten zunimmt (s. Kapitel 4). In Bild 9.9 ist das zugehörige Feld des lokalen Deviationsgrads, der aus den Konzentrationsfeldern des inerten und reaktiven Farbstoffs berechnet wird, dargestellt. Es sind die Lamellenstrukturen zu erkennen. Bild 9.9 a) zeigt eine Vergrößerung des rot umrandeten Bereichs. Dabei fällt auf, dass die Schichten in einen Kernbereich mit hohem Deviationsgrad (rot) und einen Randbereich mit kleineren Werten für den Deviationsgrad (blau) unterteilt werden können. Dies bedeutet, dass die Mikrovermischung, wie zu erwarten, in den Randbereichen der Lamellen zuerst fortschreitet. In Bild 9.10 ist für den kleinen Bildausschnitt der Deviationsgrad und die Konzentration des inerten Farbstoffs über der Bildhöhe $y$ aufgetragen. Für ausgeprägte Peaks des Konzentrationsprofils, aber unabhängig von der absoluten Konzentration, nimmt der Deviationsgrad Werte größer als 0,7 an. Es kann somit ein allgemeingültiger Filterwert für die Bemaßung der Lamellendicke gefunden werden, der unabhängig von der Verdünnung ist. Außerdem gelingt es so, schon stark mikrovermischte Bereiche von den makroskopisch segregierten zu trennen. Somit kann der makroskopische Längenmaßstab der Segregation, die Lamellendicke, unbeeinflusst von der Mikrovermischung gemessen werden. Wird der Deviationsgrad mit dem Grenzwert 0,7 binärisiert $\left(\Delta>0,7 \Rightarrow \Delta^{\prime}=1 ; \Delta<0,7 \Rightarrow \Delta^{\prime}=0\right)$, so ergibt sich das Feld des Deviationsgrads in Bild 9.9 b), das bei $x=20,97 \mathrm{~mm}$ den grau hinterlegten Bereichen in Bild 9.10 entspricht. Die Lamellendicke kann nun auch für die Lamellen, deren maximale Konzentration kleiner als $c_{\text {Filter }}$ ist gemessen werden.

Das oben beschriebene Verfahren zum Messen der Lamellendicken wird nun an unterschiedlichen Orten und zu mehreren Zeitpunkten eines Mischversuchs durchgeführt. Daraus wird eine gemittelte Lamellendicke berechnet, die repräsentativ für die gewählten Einstellungen ist. Variiert werden der Massenanteil der Zellulose (und damit die Viskosität), die Drehzahl des Rührorgans und der Zugabeort. In Bild 9.11 ist die gemittelte Lamellendicke über der Rührerdrehzahl mit der Viskosität und der Zugabeposition als Parameter dargestellt. Die Fehlerbalken geben die Standardabweichung der gemessenen Werte an. Aus dem Diagramm ergeben sich drei wesentliche Schlussfolgerungen: 

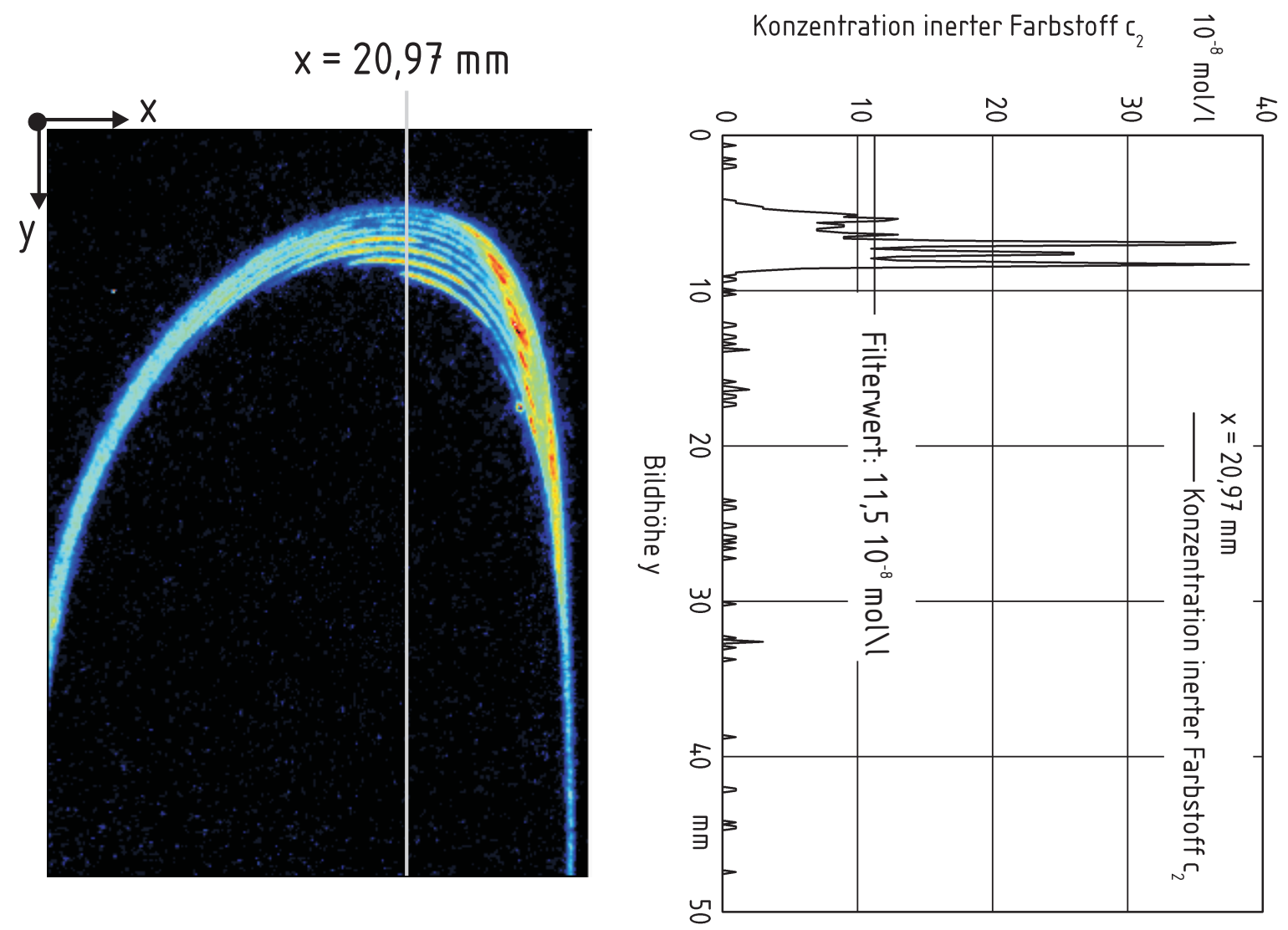

Bild 9.8: Konzentrationsfeld und- profil des inerten Farbstoffs

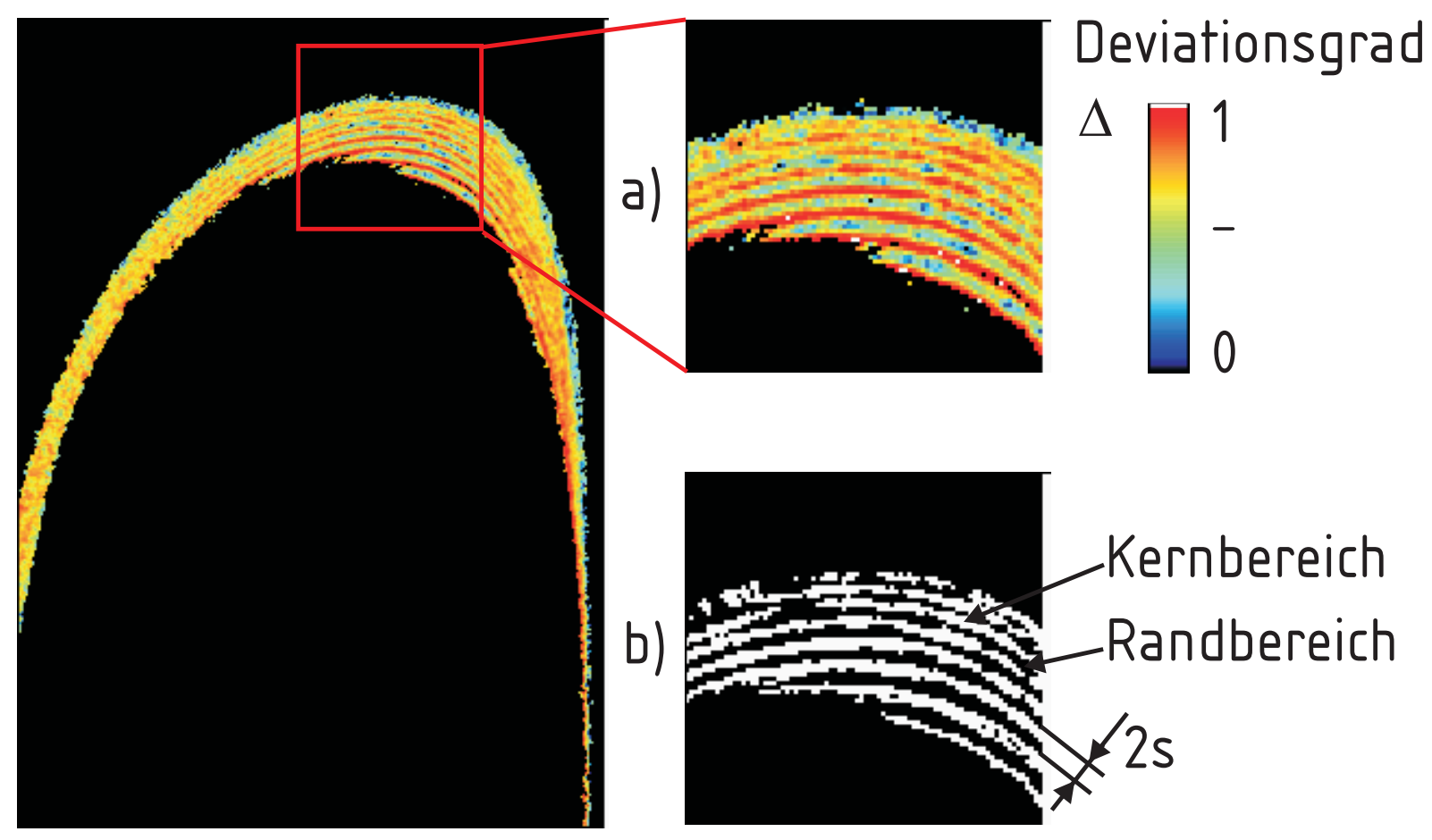

Bild 9.9: Feld des Deviationsgrads und Binärisierung mit Grenzwert $\Delta=0,7$ 


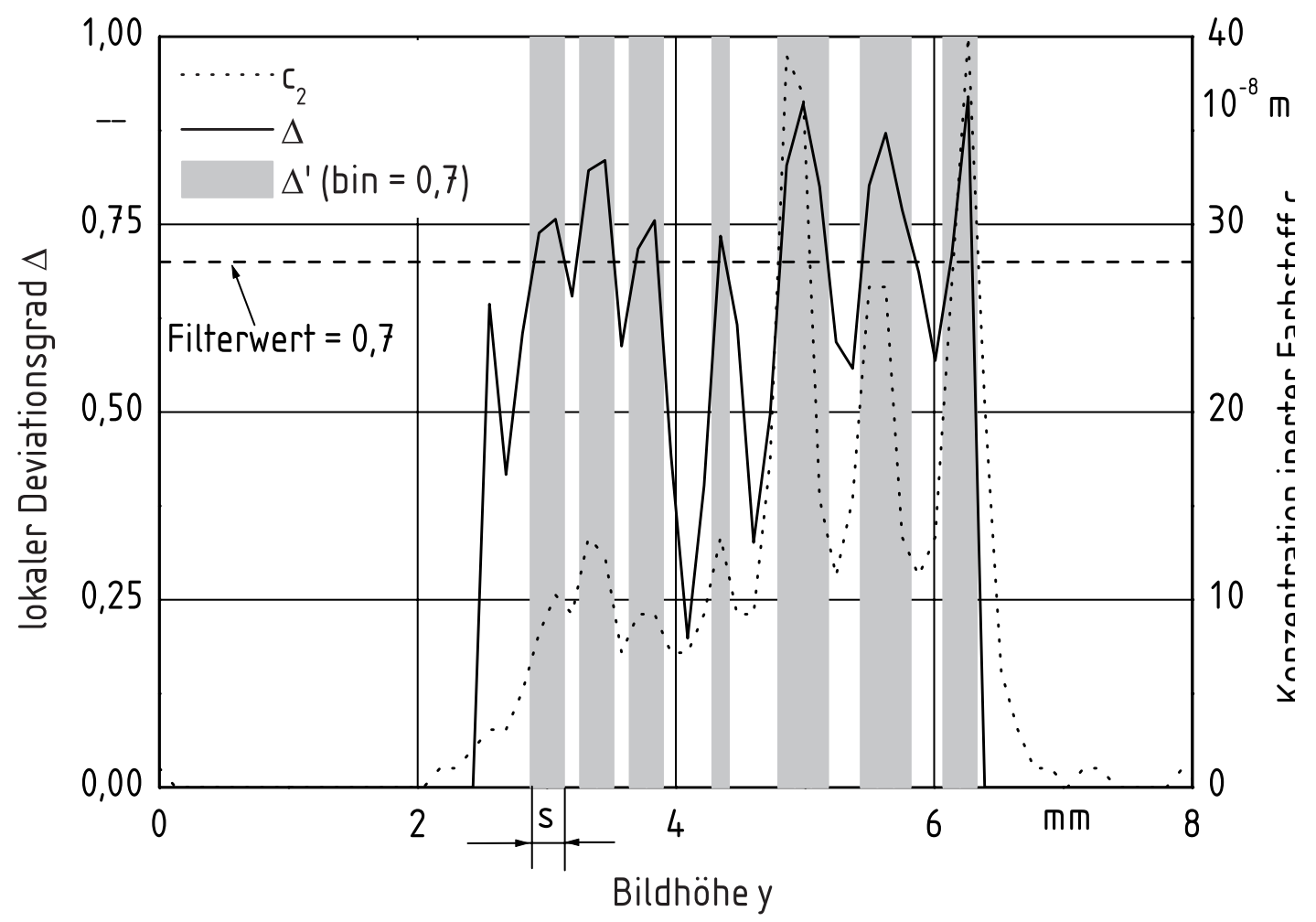

Bild 9.10: Lokaler Deviationsgrad und Konzentration des inerten Farbstoffs in Abhängigkeit von der Bildhöhe

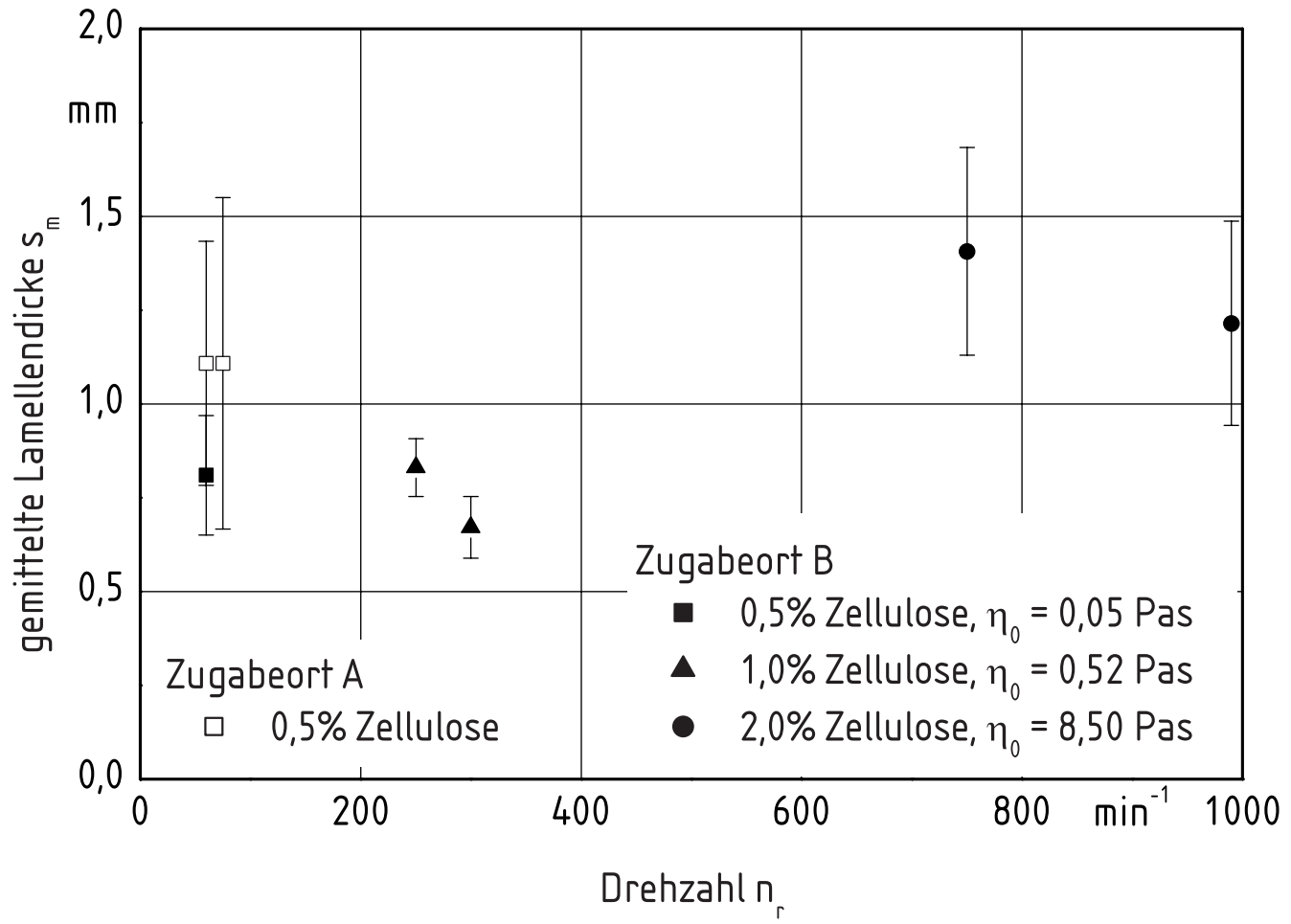

Bild 9.11: Gemittelte Lamellendicke in Abhängigkeit von der Rührerdrehzahl 
- Bei konstanter Viskosität der wässrigen Zelluloselösung nimmt die Lamellendicke mit zunehmender Drehzahl ab.

Diese Aussage gilt nur für den Zugabeort 'B' nahe der Rührerwelle. Die Lamellendicke nimmt ab, da aufgrund der größeren Strömungsgeschwindigkeit ein schnellerer Transport der Fluidelemente in Richtung der Zonen hoher Deformationsgeschwindigkeiten in der Rührerebene erfolgt. Dieser Transport ist für die Zugabeposition 'A' im Zentrum des Ringwirbels sehr eingeschränkt (s. Kapitel 9.2). Der Farbstoff verbleibt hier in kompakter Form nahe dem Zentrum und wird kaum in radialer oder axialer Richtung transportiert sondern überwiegend in Umfangsrichtung geschert. Somit hat auch die erhöhte Strömungsgeschwindigkeit kaum Einfluss auf die Mischwirkung, und die Lamellendicke ist für den Zugabeort 'A' nahezu unabhängig von der Drehzahl.

- Mit zunehmender Viskosität der wässrigen Zelluloselösungen nimmt die Lamellendicke zu.

Dieser Effekt ist besonders für die beiden höher viskosen wässrigen Zelluloselösungen zu beobachten. Die erhöhte viskose Dissipation verursacht eine schwächere Sekundärströmung verglichen zur Hauptströmung (s. Kapitel 7.5). Der Mischeffekt durch das Strecken und Falten in der Sekundärströmung wird somit mit zunehmender Viskosität geringer. Dies verursacht eine erhöhte Lamellendicke. Wie schon in Kapitel 9.3 qualitativ beschrieben, ist für die Zelluloselösung mit $\xi_{3}=2,0 \%$ eine völlig andere Lamellenstruktur zu beobachten als für die anderen beiden Zelluloselösungen. Der Transport in Richtung der Zonen hoher Deformationsgeschwindigkeiten in der Rührerebene ist stark eingeschränkt, so dass die Lamellen nicht so oft geschert werden und die Lamellendicke deutlich größer ist.

- Die Lamellendicke ist von der Zugabeposition abhängig.

Die Lamellendicke ist für den Zugabeort nahe dem Ringwirbelzentrum ('A') größer als für die Zugabe nahe der Rührerwelle ('B'). Wie schon in Kapitel 9.2 und im ersten Punkt erläutert, sind die Geschwindigkeitskomponenten in axialer und radialer Richtung, also die Sekundärströmung, im Ringwirbelzentrum nahezu Null. Es bildet sich somit eine Totzone aus, aus der nur ein sehr geringer Stoffaustausch erfolgen kann. Dementsprechend sind die Lamellendicken für eine Farbstoffzufuhr in dieser Totzone deutlich größer als für eine Zugabe in Bereiche hoher axialer Strömungsgeschwindigkeiten.

Die Auftragung der Lamellendicke über der Rührerdrehzahl ist jedoch nur von begrenzter Aussagekraft, da eine charakteristische Kennzahl der Strömung, die Reynoldszahl, sowohl 
von der Drehzahl als auch von der Viskosität beeinflusst wird. Wie in Kapitel 9.3 beschrieben, ist für eine gleichbleibende Reynoldszahl mit zunehmender Viskosität der Lösung eine deutlich höhere Drehzahl notwendig. Gleichviskose Fluide mit unterschiedlicher Reynoldszahl unterscheiden sich nur in der Rührerdrehzahl. In Bild 9.12 ist daher die gemittelte Lamellendicke in Abhängigkeit von der Reynoldszahl dargestellt. Es wurden Versuche bei $R e=40$ und $R e=52$ durchgeführt. Die oben erläuterte Abhängigkeit von der Drehzahl und der Viskosität ist allerdings nach wie vor erkennbar. Durch die Einführung der Reynoldszahl konnte kein Parameter eliminiert worden, so dass die Reynoldszahl nicht die einzige charakteristische Kennzahl darstellt und die Darstellung keine wesentlichen Vorteile bringt.

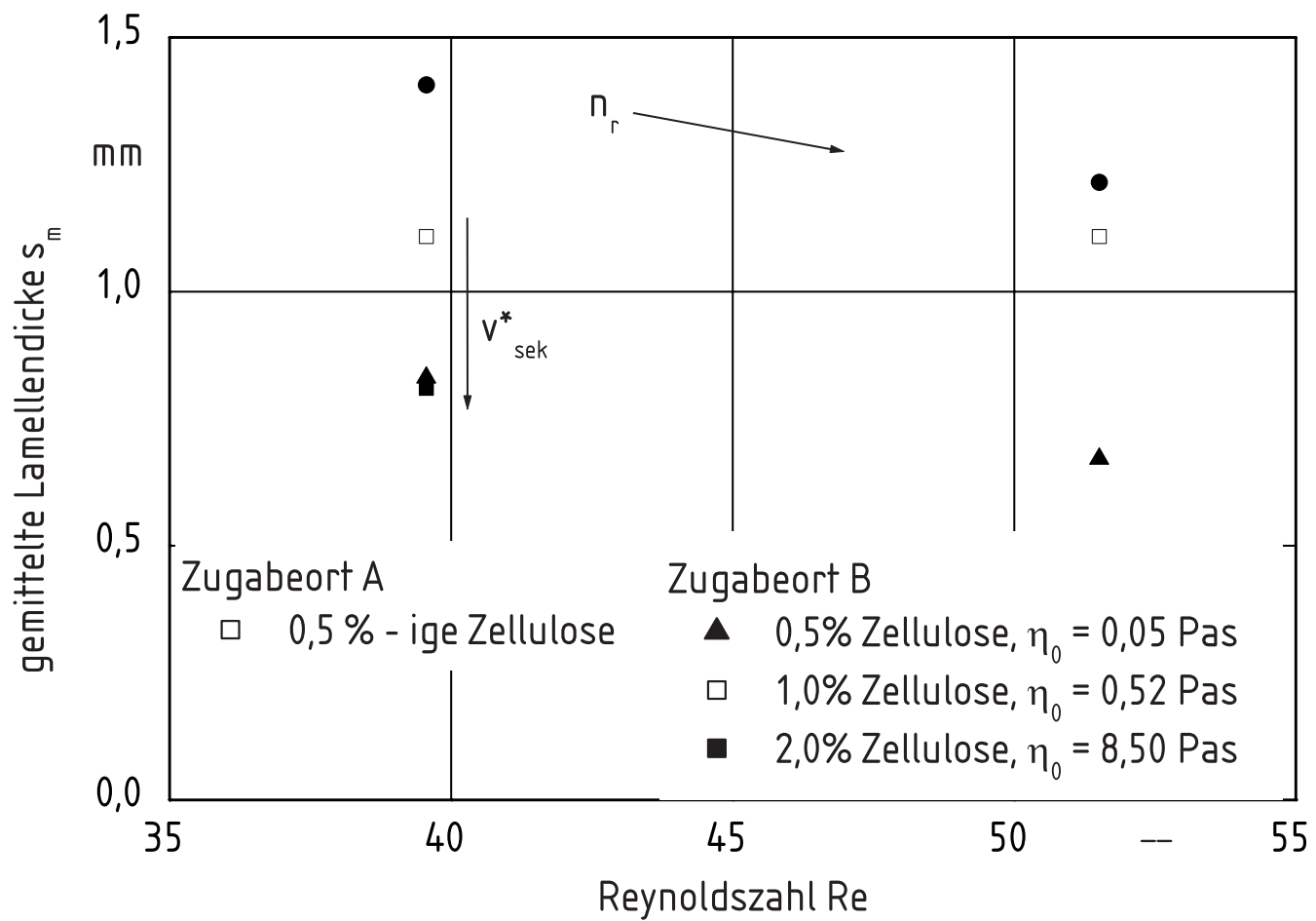

Bild 9.12: Gemittelte Lamellendicke in Abhängigkeit von der Reynoldszahl 


\section{Zusammenfassung}

Der lokale und zeitliche Ablauf von Mischvorgängen in Rührbehältern ist trotz der technischen Bedeutung für die chemische, pharmazeutische und die Lebensmittelindustrie immer noch nicht vollständig auf theoretischem Wege vorherzusagen. Die Zusammenhänge zwischen den konvektiven und diffusiven Transportströmen im Strömungsfeld und der Kinetik gleichzeitig ablaufender homogener chemischer Reaktionen ist bisher nur mit experimentellen Methoden zu ermitteln. Es sind zahlreiche Verfahren bekannt, die das indirekte Messen der Mikrovermischung gestatten. Die meisten setzen eine jedoch ideale Makrovermischung voraus. Insbesondere beim laminaren Mischen können während des Mischvorgangs stark unterschiedliche lokale Konzentrationen gleichzeitig im Behältervolumen auftreten. Für den Ablauf einer chemischen Reaktion ist die vollständige Vermischung der Reaktanden im molekularen Bereich der Ortsskalen Voraussetzung. Die Angabe eines über den Behälterinhalt gemittelten Werts der Mischgüte oder der Mischzeit ist deshalb nur von begrenztem Nutzen.

In der vorliegenden Forschungsarbeit wird simultan die Vermischung auf makroskopischen Längenskalen sowie die Zusammensetzung von fluiden Gemischen im Skalenbereich molekularer Abmessungen gemessen. Dazu wird ein Gemisch aus zwei Fluoreszenzfarbstoffen in den Behälter dosiert. Der eine Farbstoff ist inert und dient als Indikator für das Makromischen. Die Fluoreszenzintensität des anderen, reagierenden Farbstoffs wird durch eine chemische Reaktion verstärkt. Da die Reaktion eine Vermischung auf molekularer Ebene voraussetzt, dient der reagierende Farbstoff indirekt zur Visualisierung der Mikrovermischung.

Die Konzentrationsfelder werden mit Hilfe der Laserinduzierte Fluoreszenz (LIF) mit hoher zeitlicher und örtlicher Auflösung gemessen. Die Fluoreszenzfarbstoffe werden als Gemisch verwendet und müssen geeignete chemische und fluoreszenzspektroskopische Eigenschaften aufweisen. Die Berechnung der Konzentrationen aus den gemessenen Fluoreszenzintensitäten erfolgt dann unter Berücksichtigung und Korrektur der folgenden Einflussgrößen: Schwankungen der Laserleistung, ungleichmäßige Intensitätsprofile des Lasers, nicht-ideale Arbeitsweise der zur Trennung der Fluoreszenzemissionen eingesetzten optischen Filter. Aus den gemessenen Konzentrationsfeldern wird das Feld des lokalen Deviationsgrades als quantitatives Maß für die Mikrovermischung berechnet. Der lokale Deviationsgrad wird aus dem Anteil des reagierenden Farbstoffs berechnet, der lokal noch nicht umgesetzt ist. Für ein vollständig segregiertes Fluid nimmt dieser Anteil den 
Wert Eins an und fällt im Verlauf der Mischung auf den Wert Null für ein vollständig homogenes, mikrovermischtes Fluid.

Der Ablauf des Makro- und Mikromischens wird für Zelluloselösungen mit unterschiedlichen Massenanteilen und damit unterschiedlichen Viskositäten gemessen. Für konstante Reynoldszahlen ist eine starke Abhängigkeit des Prozessfortschritts von der Viskosität sowie von der Position der Farbstoffzugabe zu beobachten. Die Mikrovermischung setzt überwiegend zuerst in den Randbereichen der Lamellen ein. Dort nimmt der lokale Deviationsgrad sehr schnell Werte kleiner als 0,7 an. Mit Hilfe von Filterfunktionen kann der mikrovermischte Randbereich von dem segregierten Kernbereich der Lamellen getrennt und so die Lamellendicke als makroskopisches Längenmaß der Segregation gemessen werden. Die zeitlich und örtlich gemittelte Lamellendicke steigt mit zunehmender Viskosität der zu mischenden Fluide an.

Die Geschwindigkeits- und Viskositätsfelder werden mit dem kommerziell erhältlichen Strömungssimulationsprogramm CFX 4.4 berechnet. Die geometrische Ausdehnung der sich ausbildenden Ringwirbel nimmt mit zunehmender Viskosität ab. Auch das Verhältnis aus der maximalen Geschwindigkeit in axialer und radialer Richtung zur maximalen räumlichen Geschwindigkeit sinkt mit zunehmender Viskosität, was auf eine schwächere Sekundärströmung schließen lässt. Diese erklärt den in den Experimenten zu beobachtenden verschlechterten Ablauf der Vermischung bei hochviskosen Fluiden. 


\section{Literaturverzeichnis}

[1] J. Villermaux, R. David: Recent advances in the understanding of micromixing phenomena in stirred reactors; Chem. Eng. Commun. 21 (1983) 1-3, 105-122

[2] J. Baldyga, R. Pohorecki: Turbulent micromixing in chemical reactors - a review; Chem. Eng. J. 58 (1995), 183-195

[3] J. M. Ottino: The kinematics of mixing: stretching, chaos, and transport; Cambridge University Press, Cambridge, 1989

[4] R. Geisler, A. Mersmann, H. Voit: Makro- und Mikromischen im Rührkessel; Chem. Ing. Tech. 60 (1988) 12, 947-955

[5] P. V. Danckwerts: The effect of incomplete mixing on homogeneous reaction; Chem. Eng. Sci. 8 (1958), 93-102

[6] W. Gerlinger, K. Schneider, H. Bockhorn: Direkte numerische Simulation von Mischung und Turbulenz in zweidimensionalen Strömungen; Chem. Ing. Tech. 72 (2000) 6, 618-621

[7] W. Gerlinger, K. Schneider, L. Falk, H. Bockhorn: Numerical simulation of the mixing of passive and reactive scalars in two-dimensional flows dominated by coherent vortices; Chem. Eng. Sci. 55 (2000), 4255-4269

[8] R. O. Fox: Computational models for turbulent reacting flow; Cambridge University Press, Cambridge, 2003

[9] J. Baldyga, J. R. Bourne: Turbulent mixing and chemical reaction; John Wiley \& Sons Ltd., Chichester, 1999

[10] R. A. Bakker: Micromixing in chemical reactors: models, experiments and simulations; Dissertation, TU Delft, 1996

[11] J. Baldyga, J. R. Bourne: A fluid mechanical approach to turbulent mixing and chemical reaction; Chem. Eng. Commun. 28 (1984), 243-258

[12] J. Baldyga, J. R. Bourne, B. Walker: Non-isothermal micromixing in turbulent liquids: Theory of experiment; Can. J. Chem. Eng. 76 (1998), 641-649

[13] J. Baldyga, J. R. Bourne, S. J. Hearn: Interaction between chemical reactions and mixing on various scales; Chem. Eng. Sci. 52 (1997) 4, 457-466

[14] R. A. Bakker, H. E. A. van den Akker: Computational study of chemical reactors on the basis of micromixing models; Chem. Eng. Res. \& Des., Part A: Trans IChemE 72 (1994), 733-738 
[15] R. A. Bakker, H. E. A. van den Akker: A Lagrangian description of micromixing in a stirred tank reactor using 1D-micromixing models in a CFD flow field; Chem. Eng. Sci. 51 (1996) 11, 2643-2648

[16] A. J. Harry, J. I. G. Pearson, R. Potter: Development of an in-situ concentration measurement technique to investigate vertical mixing processes in the coastal zone; Hydrodynamics (1996), 1007-1012

[17] J. M. Ottino: Mixing and chemical reactions - a tutorial; Chem. Eng. Sci. 49 (1994) 24A, 4005-4027

[18] J. M. Ottino, W. E. Ranz, C. W. Macosko: A lamellar model for analysis of liquidliquid mixing; Chem. Eng. Sci. 34 (1979), 877-890

[19] I. Houcine, E. Plasari, R. David, J. Villermaux: Feedstream jet intermittancy phenomenon in a continuous stirred tank reaktor; Chem. Eng. J. 72 (1999), 19-29

[20] M. Buchmann: Simultanes Messen des laminaren Mikro- und Makromischens mit Hilfe der tomographischen Zweiwellenlängenphotometrie; VDI-Fortschritt-Berichte, VDI-Verlag, Düsseldorf, 1999

[21] D. J. Lamberto, M. M. Alvarez, F. J. Muzzio: Computational analysis of regular and chaotic mixing in a stirred tank reactor; Chem. Eng. Sci. 56 (2001), 4887-4899

[22] J. M. Zalc, E. S. Szalai, M. M. Alvarez, F. J. Muzzio: Using CFD to understand chaotic mixing on laminar stirred tanks; AIChE Journal 48 (2002) 10, 2124-2134

[23] E. S. Szalai, J. Kukura, P. E. Arratia, F. J. Muzzio: Effect of hydrodynamics on reactive mixing in laminar flows; AIChE Journal 49 (2003) 1, 168-179

[24] J. M. Zalc, M. M. Alvarez, F. J. Muzzio, B. E. Arik: Extensive validation of computed laminar flow in a stirred tank with three rushton turbines; AIChE Journal 47 (2001) $10,2144-2154$

[25] M. Kraume (Hrsg.): Mischen und Rühren: Grundlagen und moderne Verfahren; Wiley-VCH, Weinheim, 2003

[26] R. Mann, S. K. Pillai, A. M. el-Hamouz, P. Ying, A. Togatorop, R. B. Edwards: Computational fluid mixing for stirred vessels: progress from seeing to believing; Chem. Eng. J. 59 (1995), 39-50

[27] M. Käppel: Entwicklung und Anwendung einer Methode zur Messung des Mischungsverlaufs bei Flüssigkeiten; Dissertation, TU München, 1976

[28] J. W. Hiby: Decolouring of chemical indicators: application and limitations; 6th European Conference on Mixing, Pavia, Italien (1988) 123-128

[29] H. Henzler: Untersuchungen zum Homogenisieren von Gasen und Flüssigkeiten; VDI-Forschungsheft, 1978

[30] D. J. Lamberto, M. M. Alvarez, F. J. Muzzio.: Experimental and computational investigation of the laminar flow structure in a stirred tank; Chem. Eng. Sci. 54 (1999), 919-942 
[31] D. J. Lamberto, F. J. Muzzio, P. D. Swanson, A. L. Tonkovich: Using time-dependent RPM to enhance mixing in stirred vessels; Chem. Eng. Sci. 51 (1996) 5, 733-741

[32] G. Ascanio, S. Foucault, P. A. Tanguy: New chaotic approach for mixing shearthinning fluids in stirred tanks; 4th ASME-JSME Joint Fluids Engineering Conference, Honolulu, HI, USA (2003), 1-5

[33] J. Baldyga, J. R. Bourne: Simplification of micromixing calculations II. new applications; Chem. Eng. J. 42 (1989), 93-101

[34] S. Yu: Micromixing and parallel reactions; Dissertation, ETH Zürich, 1993

[35] D. Gouye, R. David, J. Villermaux: Untersuchung der lokalen Mikromischverhältnisse in Rührbehältern durch eine chemische Methode; Chem. Ing. Tech. 59 (1987) $3,254-255$

[36] M. Assirelli, W. Bujalski, A. W. Nienow, A. Eaglesham: Study of micromixing in a stirred tank using a rushton turbine: Comparison of feed position and other mixing devices; CHISA, Prag, Czechische Republik (2002)

[37] J. Baldyga, J. R. Bourne: The effect of micromixing on parallel reactions; Chem. Eng. Sci. 45 (1990) 4, 907-916

[38] J. R. Bourne, C. P. Hilber, S. Petrozzi: Influence of viscosity on micromixing in turbulent flows; Chem. Eng. Process. 25 (1989), 133-139

[39] J. M. Rousseaux, L. Falk, H. Muhr, E. Plasari: Micromixing efficiency of a novel sliding-surface mixing device; AIChE Journal 45 (1999) 10, 2203-2213

[40] M. C. Fournier, L. Falk, J. Villermaux: A new parallel competing reaction system for assessing micromixing efficiency-determination of micromixing time by a simple mixing model; Chem. Eng. Sci. 51 (1996) 23, 5187-5192

[41] P. Guichardon, L. Falk: Characterization of micromixing efficiency by the iodideiodate reaction system. Part I: experimental procedure; Chem. Eng. Sci. 55 (2000), 4233-4243

[42] F. Mayinger, O. Feldmann: Optical measurements- techniques and applications; Springer-Verlag, Berlin, 2. korr. Auflage, 2001

[43] P. J. Holden, M. Wang, R. Mann, F. J. Dickin, R. B. Edwards: Imaging stirred-vessel macromixing using electrical resistance tomography; AIChE Journal 44 (1998) 4, $780-790$

[44] W. Haarde: Das Vermischen mit Hilfe von Flüssigkeitsstrahlen; Dissertation, Universität Hannover, 1989

[45] W. Haarde, D. Mewes: Das Vermischen geringer Stoffmengen in großvolumigen Lagertanks und chemischen Reaktoren; Chem. Ing. Tech. 62 (1990) 1, 52-53

[46] R. Renz: Das diskontinuierliche Vermischen von Flüssigkeitsstrahlen in zylindrischen Behältern; Dissertation, Universität Hannover, 1992

[47] R. Renz, D. Mewes: Experimentelle Untersuchungen zur Ausbreitung von Reaktionsstoppern in Lagertanks; Chem. Ing. Tech. 64 (1992) 6, 565-567 
[48] M. Buchmann, D. Mewes: Measurement of the local intensities of segregation with the tomographical Dual Wavelength Photometry; Can. J. Chem. Eng. 76 (1998) 3, $626-630$

[49] K. Sato, N. Kasagi, Y. Suzuki: Combined velocity and scalar field measurements with the simultaneous use of PIV and scanning LIF; Transport Phenomena in Thermal Science and Prosess Engineering (1997), 541-546

[50] S. Deusch, T. Dracos: Time resolved 3D passive scalar concentration-field imaging by laser induced fluorescence (LIF) in moving liquids; Meas. Sci. Technol. 12 (2001) $2,188-200$

[51] E. van Vliet, S. M. van Bergen, J. J. Derksen, L. M. Portela, H. E. A. van den Akker: Time-resolved, 3D, laser-induced fluorescence measurements of fine-structure passive scalar mixing in a tubular reactor; Experiments in Fluids 37 (2004) 1, 1-21

[52] W. J. A. Dahm, L. K. Su, K. B. Southerland: A scalar imaging velocimetry technique for fully resolved four dimensional vector velocity field measurement in turbulent flows; Phys. Fluids A (Fluid Dynamics) 4 (1992) 10, 2191-2206

[53] C. André, R. David, J. C. André, J. Villermaux: A new fluorescence method for measuring cross-fluctuations of two non-reactive components in a mixing Device; Chem. Eng. Technol. 15 (1992), 182-185

[54] R. David, C. André: Comparisation of single and double inert tracer fluctuation measurements in a Continuous Stirred Tank; Chem. Eng. Technol. 16 (1993), 234237

[55] C. André, R. David, J. Villermaux: Measurement of concentration fluctuations during mixing in a stirred tank and in a channel flow by laserinduced fluorescence spectroscopy

[56] M. F. W. Distelhoff, A. J. Marquis: Scalar mixing in the vicinity of two disk turbines and two pitched blade impellers; Chem. Eng. Sci. 55 (2000) 10, 1905-1920

[57] M. F. W. Distelhoff, A. J. Marquis: A LIF line scan system for the measurement of scalar concentration in a continiously operated stirred tank; Experiments in Fluids 25 (1998), 77-88

[58] F. W. M. Distelhoff, A. J. Marquis: Scalar mixing measurements in continuously operated stirred tank; Can. J. Chem. Eng. 79 (2001) 2, 187-202

[59] W. J. A. Dahm, K. B. Southerland, K. B. Buch: Direct, high resolution, fourdimensional measurements of the fine scale structure of $S c \gg 1$ molecular in turbulent flows; Phys. Fluids A (Fluid Dynamics) 3 (1991) 5, 1115-1127

[60] W. J. A. Dahm, K. B. Southerland, K. A. Buch: Four-dimensional laser induced fluorescence measurements of conserved scalar mixing in turbulent flows; 5th International Symposium on Applications of laser techniques to fluid mechanics, Lissabon, Portugal (1990), 3

[61] I. Houcine, H. Vivier, E. Plasari, R. David, J. Villermaux: Planar laser induced fluorescence technique for measurements of concentration fields in continuous stirred tank reactors; Experiments in Fluids 22 (1996), 95-102 
[62] I. Houcine, B. Marcant, H. Vivier, E. Plasari, R. David, J. Villermaux: Comparison of mixing action of several stirrers by laser sheet visualisation and image processing; IChemE Symp. Series 136 (1994), 97-104

[63] D. R. Unger, F. J. Muzzio: Laser Induced Fluorescence technique for the quantification of mixing in impinging jets; AIChE Journal 45 (1999) 12, 2477-2486

[64] J. A. Bellerose, C. B. Rogers: Measuring mixing and local $p H$ through laser induced fluorescence; Laser Anemometry 191 (1994), 217-220

[65] B. M. Cetegen, N. Mohamad: Experiments on liquid mixing and reaction in a vortex; Journal of Fluid Mechanics 249 (1993), 391-414

[66] S. D. Hong, Y. Sugii, K. Okamoto, H. Madarame: Evaluation on the chemically reacting liquid round jet by LIF technique; 10th International Symposium on Flow Visualization, Kyoto, Japan (2002) F0275

[67] F. Guillard, C. Trägardh, L. Fuchs: New image analysis methods for the study of mixing patterns in stirred tanks; Can. J. Chem. Eng. 78 (2000) 2, 273-285

[68] F. Guillard, C. Trägardh, L. Fuchs: A study of turbulent mixing in a turbine-agitated tank using a fluorescence technique; Experiments in Fluids 28 (2000) 3, 225-235

[69] F. Guillard, C. Trägardh, L. Fuchs: A study on the instability of coherent mixing structures in a continuously stirred tank; Chem. Eng. Sci. 55 (2000) 23, 5657-5670

[70] M. M. Alvarez, J. M. Zalc, T. Shinbrot, F. J. Muzzio: Mechanisms of mixing and creation of structure in laminar stirred tanks; AIChE Journal 48 (2002) 10, 21352148

[71] J. R. Coppeta, C. B. Rogers: Mixing measurements using Laser Induced Fluorescence; AIAA 95-0167 (1994), 1-5

[72] J. R. Coppeta, C. B. Rogers: A quantitative mixing analysis using fluorescent dyes; AIAA 96-0539 (1995), 1-8

[73] J. R. Coppeta, C. B. Rogers: Dual emission laser induced fluorescence for direct planar behaviour measurements; Experiments in Fluids 25 (1998), 1-15

[74] J. Sakakibara, R. J. Adrian: Whole field measurement of temperature in water using two-color laser induced fluorescence; Experiments in Fluids 26 (1999), 7-15

[75] T. Makino, N. Ohmura, K. Kataoka: Observation of isolated mixing regions in a stirred vessel; Journal of Chemical Engineering of Japan 34 (2001) 5, 574-578

[76] H. Hu, T. Saga, T. Kobayashi, N. Taniguchi: Research on the vortical and turbulent structures in the lobed jet flow using laser induced fluorescense and particle image velocimetry techniques; Meas. Sci. Technol. 11 (2000), 698-711

[77] C. Arcoumanis, J. J. McGuirk, J. M. L. M. Palma: On the use of fluorescence dyes for concentration measurements in water flows; Experiments in Fluids 10 (1990), $177-180$

[78] D. A. Walker: A fluorescence technique for measurement of concentration in mixing liquids; Journal of Physics E: Scientific instruments 20 (1987) 2, 217-224 
[79] A. W. Law, H. Wang: Measurement of mixing processes with combined digital particle image velocimetry and planar laser induced fluorescence; Experimental Thermal and Fluid Science 22 (2000) 3/4, 213-229

[80] H. Höcker, G. Langer, U. Werner: Der Leistungsbedarf von Rührern in nichtnewtonschen Flüssigkeiten; Chem. Ing. Tech. 52 (1980) 11, 916-917

[81] I. Houcine, E. Plasari, R. David: Effects of the stirred tanks design on power consumption and mixing temperature in liquid phase; Chem. Eng. Technol. 23 (2000) 7, 605-613

[82] P. Vrabel, R. G. J. M. van der Lans, K. C. H. A. M. Luyben, L. Boon, A. W. Nienow: Mixing in large-scale vessels stirred with multiple radial or radial and axial up-pumping impellers: modelling and measurements; Chem. Eng. Sci. 55 (2000) 23, $5881-5896$

[83] M. Bouaif, M. Roustan: Power consumption, mixing time and homogenisation energy in dual-impeller agitated gas-liquid reactors; Chem. Eng. Process. 40 (2001), $87-95$

[84] Y. Sumi, M. Kamiwano: Development and mixing characteristics of a multistage impeller for agitating highly viscous fluids; Journal of Chemical Engineering of Japan 34 (2001) 4, 485-492

[85] L. Perse, M. Zumer: Mixing studies- the effect of non-Newtonian fluid behaviour; CHISA, Prag, Czechische Republik (2002)

[86] Y. Hirata, Y. Aoshima: Flow characteristics and power consumption in an agitated shear-thinning plastic fluid; 8th European Conference on Mixing, Cambridge, UK (1994), 415-421

[87] A. Bakker, A. H. Haidari, L. M. Oshinowo: Realize greater benefits from CFD; Chemical Engineering Progress 97 (2001) 3, 45-53

[88] A. Souvaliotis, S. C. Jana, J. M. Ottino: Potentialities and limitations of mixing simulations; AIChE Journal 41 (1995) 7, 1605-1621

[89] A. Birtigh, G. Lauschke, W. F. Schierholz, D. Beck, C. Maul, N. Gilbert, H.-G. Wagner, C. Y. Werninger: CFD in der chemischen Verfahrenstechnik; Chem. Ing. Tech. 72 (2000) 3, 175-193

[90] Y. Zhao, R. S. Brodkey, S. Nakamura: Study of 3D mixing processes by numerical and experimental approaches; ASME Fluids Engineering Division Summer Meeting, Montreal, Kanada (2002)

[91] J. Revstedt, L. Fuchs: Large eddy simulation of flow in stirred vessels; Chem. Eng. Technol. 25 (2002) 4, 443-446

[92] A. K. Sahu, P. Kumar, A. W. Patwardhan, J. B. Joshi: CFD modelling and mixing in stirred tanks; Chem. Eng. Sci. 54 (1999), 2285-2293

[93] J. Sheng, H. Meng, R. O. Fox.: Validation of CFD simulations of stirred tank using particle image Velocimetry Data; Can. J. Chem. Eng. 76 (1998), 611-625 
[94] P. Pakdel, G. H. McKinley: Digital particle imaging velocimetry of viscoelastic fluids; AIChE Journal 43 (1997) 2, 289-302

[95] K. Soroka, R. S. Vithanage, D. A. Phillips, B. Walker, P. K. Dasgupta: Fluorescence properties of metal complexes of 8-hydroxyquinoline-5-sulfonic acid and chromatographic applications; Anal. Chem. 59 (1987) 4, 629-636

[96] P. Bryanston-Cross, M. Burnett, B. Timmerman, W. K. Lee, P. Dunkley: Intelligent diagnostic optics for flow visualization; Optics and Laser Technology 32 (2000), $641-654$

[97] H. D. Laufhütte, A. Mersmann: Die Laser-Doppler-Velocimetrie - ein geeignetes Meßverfahren zur Ermittlung der Strömungsverhältnisse in gerührten Fluiden; Chem. Ing. Tech. 56 (1984) 11, 862-863

[98] R. S. Brodkey: Turbulence in mixing operations; Academic Press, New York, 1975

[99] H. D. Laufhütte, A. Mersmann: Die lokale Energiedissipation im turbulent gerührten Fluid und ihre Bedeutung für die verfahrenstechnische Auslegung von Rührwerken; Chem. Ing. Tech. 57 (1985) 12, 1104-1105

[100] F. Werner, A. B. Mersmann: An engineering approach to turbulence; 8th European Conference on Mixing, Cambridge, UK (1994), 129-136

[101] G. Montante, K. C. Lee, A. Brucato, M. Yianneskis: Numerical simulations of the dependency of flow pattern on impeller clearance in stirred vessels; Chem. Eng. Sci. 56 (2001) 12, 3751-3770

[102] I. P. T. Moore, G. Cossor, M. R. Baker: Velocity distrubutions in a stirred tank containing a yield stress fluid; Chem. Eng. Sci. 50 (1995) 15, 2467-2481

[103] M. Schäfer, M. Yianneskis, P. Wächter, F. Durst: Trailing vortices around a $45^{\circ}$ pitched-blade impeller; AIChE Journal 44 (1998) 6, 1233-1246

[104] K. V. Sharp, R. J. Adrian: PIV study of small-scale flow structure around a rushton turbine; AIChE Journal 47 (2001) 4, 766-778

[105] V. V. Ranade, M. Perrard, N. L. Sauze, C. Xuereb, J. Bertrand: Trailing vortices of rushton turbine: PIV measurements and CFD simulations with snapshot approach; Chem. Eng. Res. Des. 79 (2001) 1, 3-12

[106] R. Escudié, A. Liné: Experimental analysis of hydrodynamics in a radially agitated tank; AIChE Journal 49 (2003) 3, 585-603

[107] S. Baldi, M. Yianneskis: On the quantification of energy dissipation in the impeller stream of a stirred vessel from fluctuating velocity gradient measurements; Chem. Eng. Sci. 59 (2004), 2659-2671

[108] K. D. Hinsch: Holographic Particle Image Velocimetry; Meas. Sci. Technol 13 (2002), R61-R72

[109] G. Papadopoulos, K. J. Hammad: Time-resolved PIV measurements within a triple impeller stirred-tank; 4th ASME-JSME Joint Fluids Engineering Conference, Honolulu, HI, USA (2003), 1-8 
[110] J. W. Hiby: Definition und Messung der Mischgüte in flüssigen Gemischen; Chem. Ing. Tech. 51 (1979) 7, 704-709

[111] G. Talsky: Derivative spectrophotometry: low and higher order; Wiley-VCH, Weinheim, 1994

[112] Y. Arakawa, O. Wada: Extraction and fluorimeric determination of Organotin compounds with Morin; Anal. Chem. 55 (1983) 12, 1901-1904

[113] M. Eberhard, P. Erne: Kinetics of Calcium binding to fluo-3 determined by stoppedflow fluorescence; Biochem. Biophys. Res. Commun. 163 (1989), 309-314

[114] H. Song, M. R. Bringer, J. D. Tice, C. J. Gerdts, R. F. Ismagilov: Experimental test of scaling of mixing by chaotic advection in droplets moving through microfluidic channels; Applied Physics Letters 83 (2003) 22, 4664-4666

[115] G. Peev, A. Nikolova: On the existance of an anamalously high dissolution rate in films of non-newtonian liquids with short contact times; J. Non-Newtonian Fl. Mech. 8 (1981), 319-326

[116] R. P. Haugland: Handbook of fluorescent probes and research products; Molecular Probes Inc., USA, 2002

[117] R. B. Bird, W. E. Stewart, E. N. Lightfoot: Transport Phenomena; John Wiley and Sons, New York, 1960

[118] O. Wünsch: Strömungsmechanik des laminaren Mischens; Springer Verlag, Berlin, Heidelberg, 2001

[119] H. Giesekus: Phänomenologische Rheologie: eine Einführung; Springer-Verlag, Berlin, Heidelberg, 1994

[120] CFX 4.4 User Manual; AEA Technology, Oxfordshire, 1999 


\section{Lebenslauf}

Persönliche Daten

Name

Geburtsdatum

Geburtsort

Staatsangehörigkeit

Familienstand

\section{Schulbesuch}

$1980-1986$

$1986-1993$

Mai 1993

\section{Hochschulstudium}

$1993-1999$

September 1998 - April 1999

September 1999

\section{Berufliche Tätigkeit}

November 1999 - Dezember 2004 Wissenschaftliche Mitarbeiterin am

Institut für Verfahrenstechnik, Universität Hannover Institutsleitung: Prof. Dr.-Ing. D. Mewes
Kerstin Kling

10.04.1974

Bückeburg

deutsch

unverheiratet
Grundschule und Orientierungsstufe, Obernkirchen Neues Gymnasium, Stadthagen

Abitur

Diplomstudiengang Maschinenbau an der Universität Hannover, Vertiefungsrichtung Energie- und Verfahrenstechnik

Forschungsaufenthalt an der Stanford University, Kalifornien, USA, zur Anfertigung der Diplomarbeit Diplom

Hannover, im Dezember 2004 